Supplementary information

\title{
Highly Efficient Production of Heteroarene Phosphonates by Dichromatic Photoredox Catalysis
}

Jorge C. Herrera-Luna, ${ }^{a}$ David Díaz Díaz,,${ }^{b, c}$ M. Consuelo Jiménez, ${ }^{* a}$ and Raúl PérezRuiz*a

a Departamento de Química, Universitat Politècnica de València (UPV), Camino de Vera S/N, 46022, Valencia, Spain.

b Departamento de Química Orgánica and instituto de Bio-Orgánica Antonio González, Universidad de La Laguna, Avda. Astrofísico Francisco Sánchez 3, 38206, La Laguna, Spain.

c Institut für Organische Chemie, Universität Regensburg, Universitätsstr. 31, 93053, Regensburg, Germany.

\section{TABLE OF CONTENTS}

$>$ Materials and methods

$>$ General procedure

Synthesis of LMW Gelators

$>$ Searching the optimal conditions

$>$ Cyclic Voltammetry

$>$ Kinetic studies

Frozen model reaction procedure

UV-vis absorption spectra

$>$ Trapping reaction

Sunlight irradiation and $1 \mathrm{mmol}$ scale

Oscillatory rheology

$>$ Field-emission scanning electron microscopy (FESEM)

Characterization of compounds 


\section{Materials and methods}

All reagents ( $\geq 97 \%$ purity) and solvents ( $\geq 99 \%$ purity) were purchased from commercial suppliers (Merck, TCl, Apollo Scientific, Fluorochem, Scharlab) and used as received unless otherwise indicated. Reactions were carried out in Metria $₫$-Crimp Headspace clear vial flat bottom $\left(10 \mathrm{~mL}, \varnothing 20 \mathrm{~mm}\right.$ ) sealed with Metria ${ }^{\circledR}$-aluminium crimp cap with moulded septum butyl/natural PTFE $(\varnothing 20 \mathrm{~mm})$. Irradiation was performed with a cool white LED (LED Cree MK-R, cold-white, $11.6 \mathrm{~V}, 700 \mathrm{~mA}, \mathrm{P}=8.5 \mathrm{~W}$ ). TLC was performed on commercial $\mathrm{SiO}_{2}$-coated aluminium and plastic sheets (DC60 F254, Merck). Visualization was done by UV-light $(254 \mathrm{~nm})$. Product were isolated materials after column flash chromatography or TLC on silica gel (Merck, mesh 35-70, 60 A pore size) and their corresponding yields were determined by quantitative GC-FID measurements on an Agilent 8860 GC-System with $\mathrm{N}_{2}$ as carrier gas. Dodecanenitrile was used as an internal standard in the GC-FID quantitative measurements; yield products were estimated as: [conversion $\times$ selectivity]/mass balance. Determination of purity and structure confirmation of the literature known products was performed by ${ }^{1} \mathrm{H} N M R,{ }^{13} \mathrm{C}$ NMR, ${ }^{19} \mathrm{~F},{ }^{31} \mathrm{P}$ and high-resolution mass spectrometry (HRMS) in case of unknown products. NMR spectral data were collected on a Bruker Advance $400\left(400 \mathrm{MHz}\right.$ for ${ }^{1} \mathrm{H}$, $101 \mathrm{MHz}$ for ${ }^{13} \mathrm{C}, 376 \mathrm{MHz}$ for ${ }^{19} \mathrm{~F}$ and $162 \mathrm{MHz}$ for ${ }^{31} \mathrm{P}$ ) spectrometer at $20{ }^{\circ} \mathrm{C}$. Chemical shifts are reported in $\delta / p p m$, coupling constants $\mathrm{J}$ are given in Hertz. Solvent residual peaks were used as internal standard for all NMR measurements. The quantification of ${ }^{1} \mathrm{H}$ cores was obtained from integrations of appropriate resonance signals. Abbreviations used in NMR spectra: $\mathrm{s}$ - singlet, $\mathrm{d}$ - doublet, $\mathrm{t}$ - triplet, $\mathrm{q}$ - quartet, $\mathrm{m}$ - multiplet, $\mathrm{dd}$ doublet of doublet, ddd - doublet of doublet of doublet, $\mathrm{td}$ - triplet of doublet and $\mathrm{dq}-$ doublet of quartet. HRMS was carried out was performed in the mass facility of SCSIE University of Valencia. Absorption spectra were recorded on a JASCO V-630 spectrophotometer. The fluorescence spectra were recorded on an FS5 Edinburgh instrument spectrofluorometer with a SC-05 standard cuvette holder module and an SC30 integrating sphere module. 


\section{General procedure}

A vial $(10 \mathrm{~mL})$ was charged with 9,10 -anthracenedicarbonitrile $(1.2 \mathrm{mg}, 5 \mu \mathrm{mol}, 10 \mathrm{~mol} \%$ and the correspondent gelator $(\mathrm{G} 1,10 \mathrm{mg} / \mathrm{mL})$. Anhydrous acetonitrile $(1.0 \mathrm{~mL})$ was poured and 5-chloro-2-thiophenecarbonitrile $(5.4 \mu \mathrm{L}, 50 \mu \mathrm{mol}, 1.0$ equiv.) and triethylphosphite $(45 \mu \mathrm{L}, 250 \mu \mathrm{mol}, 5.0$ equiv.) were added. Then, DIPEA (10.5 $\mu \mathrm{L}, 60$ $\mu \mathrm{mol}, 1.2$ equiv.) and dodecanenitrile $(12.0 \mu \mathrm{L}, 50 \mu \mathrm{mol}, 1.0$ equiv.) were added with 25 $\mu \mathrm{L}$ Hamilton syringe. The vial was sealed with a septum. It was heated to $150{ }^{\circ} \mathrm{C}$ with heatgun for 1.5 minutes with manual stirring until complete clear solution. The vial cooled to room temperature until gel formation was observed. The reaction was irradiated with an external LED through the plain bottom side of the vial at $23{ }^{\circ} \mathrm{C}$ during the corresponding time. Then, brine $(2 \mathrm{~mL})$ was added, and the aqueous phase was extracted with ethyl acetate $(1 \mathrm{~mL})$. The reaction was monitored by GC-FID analysis. The organic phase was dried over anhydrous sodium sulfate, filtered from the drying agent, and concentrated in vacuo. The crude was purified via TLC plastic sheet or flash column chromatography using a hexane/ethyl acetate mixture as the mobile phase. Note: The gelator can be easily separated by filtration and reused in subsequent experiments without any detriment of its gelation properties.

\section{Lamp emission: cold white light LED}
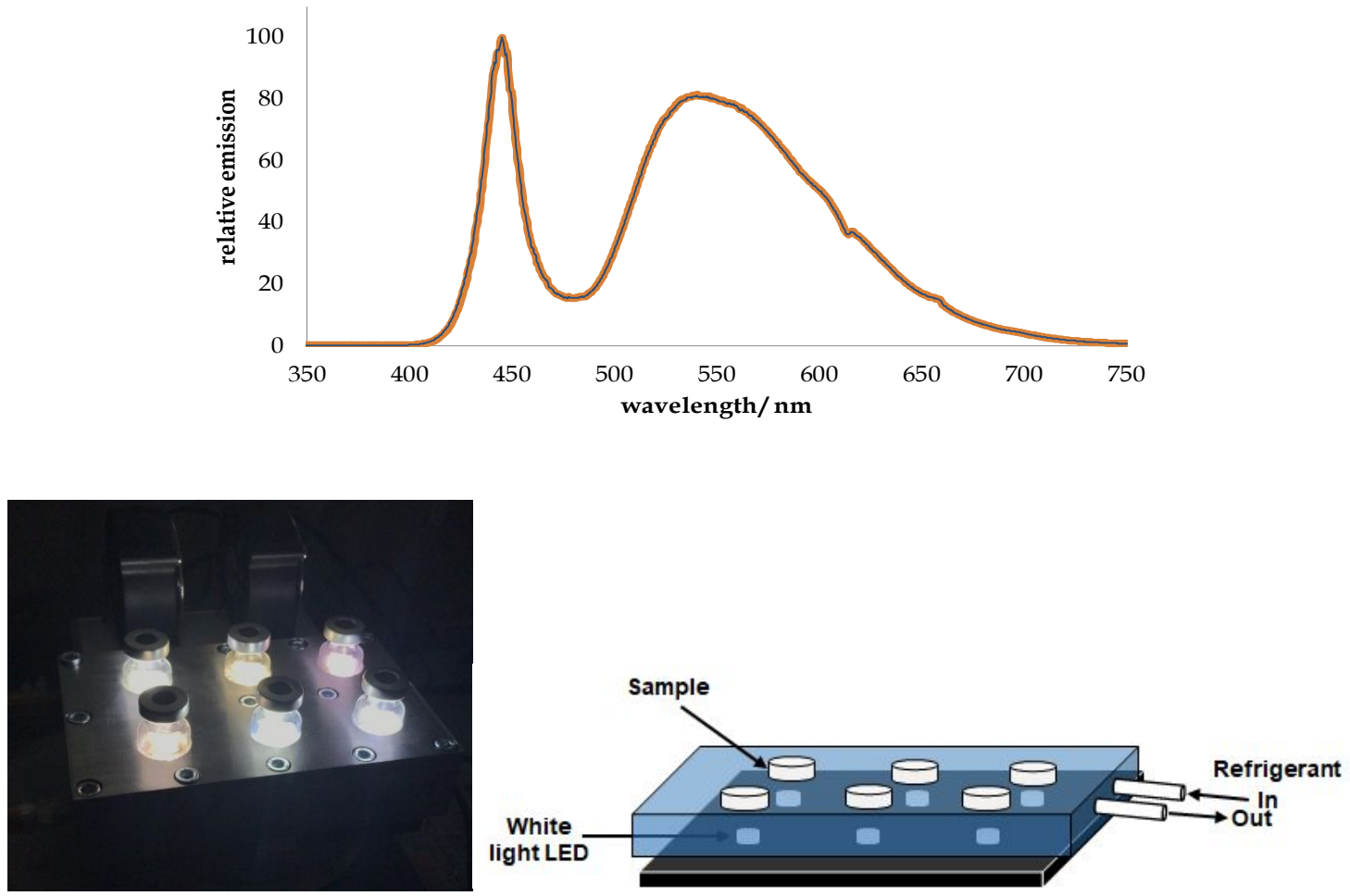

Chart S1. Lamp emission spectrum of the white light LEDs and irradiation setup. 


\section{Synthesis of Low Molecular Weight Gelators (LMW Gelators)}

N,N'-Bis(octadecyl)-L-Boc-glutamic Diamide (G1).

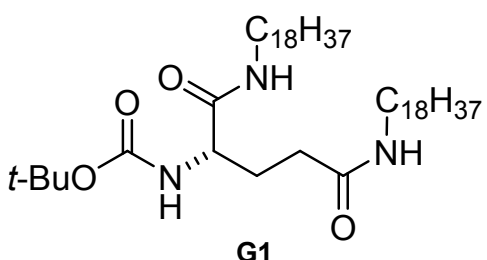

Boc-glutamic acid (0.01 mol, 1.0 equiv) and octadecylamine ( $0.02 \mathrm{~mol}, 2.0$ equiv) in dichloromethane $(200 \mathrm{~mL})$ were mixed. Then, 1-ethyl-3-(3(dimethylamino)propyl)carbodiimide hydrochloride (EDC $\cdot \mathrm{HCl})(0.022 \mathrm{~mol}, 2.2$ equiv) was added to the mixture and stirred at RT for $72 \mathrm{~h}$. The obtained white solid was isolated by filtration and washed three times with dichloromethane. The crude product was dissolved in THF and precipitated by water. A fine white solid was obtained (80\%). Following the procedure reported previously Soft Matter 2007, 3, 1312-1317.

${ }^{1} \mathrm{H}$ NMR (400 MHz CDCl $\left.{ }_{3}\right) \delta 6.69(\mathrm{br}, 1 \mathrm{H}), 6.32(\mathrm{br}, 1 \mathrm{H}), 5.77$ (br, 1H), $4.08(\mathrm{br}, 1 \mathrm{H})$, 3.26-3.22 (m, 4H), 2.41-2.27 (m, 2H), 2.06-1.93 (m, 2H), 1.58-1.46 (m, 4H), $1.43(\mathrm{~s}$, $9 \mathrm{H}), 1.25(\mathrm{~s}, 60 \mathrm{H}), 0.87(\mathrm{t}, 6 \mathrm{H}) \mathrm{ppm}$.

(S,S)-Dodecyl-3-[2(3-dodecyl-ureido)cyclohexyl]urea (G2).

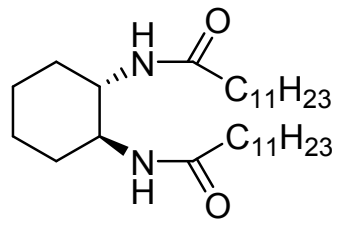

G2

A solution of dodecylisocyanate (15 mmol, 2.0 equiv) in toluene (20 $\mathrm{mL})$ was slowly added to a solution of $(S, S)-1,2-$ cyclohexyldiamine $(7 \mathrm{mmol}, 1.0$ equiv) in toluene $(100 \mathrm{~mL})$. The reaction mixture was stirred for $16 \mathrm{~h}$ at $\mathrm{RT}$ and $2 \mathrm{~h}$ at $100^{\circ} \mathrm{C}$. After cooling to $\mathrm{RT}$, the gel-like reaction mixture was filtered to give a white waxy solid. The waxy solid was further stirred for $16 \mathrm{~h}$ with dichloromethane $(50 \mathrm{~mL})$ and collected by filtration. This procedure was repeated with diethyl ether. After drying, a white solid was obtained (70\%). Following the procedure reported previously Chem. - Eur. J. 1999, 5, 937-950.

${ }^{1} \mathrm{H}$ NMR $\left(400 \mathrm{MHz}, \mathrm{CDCl}_{3}\right) \delta 5.18(\mathrm{br}, 2 \mathrm{H}), 4.67(\mathrm{br}, 2 \mathrm{H}), 3.42(\mathrm{~m}, 2 \mathrm{H}), 3.08(\mathrm{~m}, 4 \mathrm{H})$, $2.03(\mathrm{~d}, 2 \mathrm{H}), 1.72(\mathrm{~m}, 6 \mathrm{H}), 1.45(\mathrm{~m}, 4 \mathrm{H}), 1.25(\mathrm{~s}, 36 \mathrm{H}), 0.87(\mathrm{t}, 6 \mathrm{H}) \mathrm{ppm}$.

\section{1,3,2,4-Dibenzylidene-D-sorbitol (G3).}<smiles>OC[C@@H](O)[C@H]1OC(c2ccccc2)O[C@@H]2COC(c3ccccc3)OC21</smiles>

Commercially available. 
Searching the optimal conditions

Table S1. Optimizing the reaction conditions ${ }^{a}$

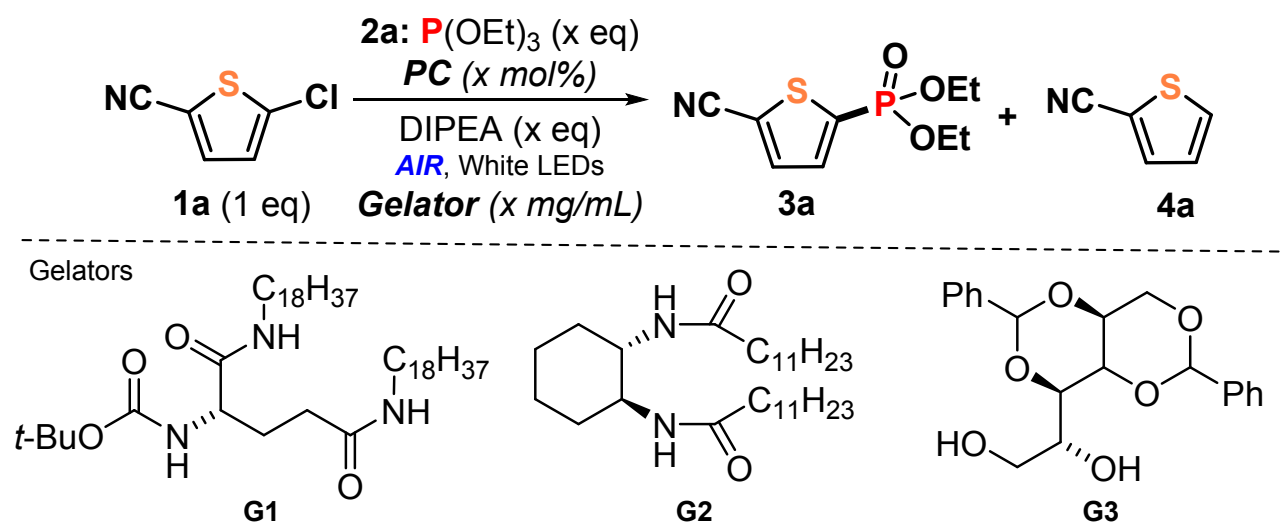

\begin{tabular}{|c|c|c|c|c|c|c|c|}
\hline Entry & $\begin{array}{l}2 a \\
(e q)\end{array}$ & $\begin{array}{c}\text { DIPEA } \\
\text { (eq) }\end{array}$ & $\begin{array}{c}\mathbf{P C}^{b} \\
(\mathrm{~mol} \%)\end{array}$ & $\begin{array}{c}\mathbf{G}^{c} \\
(\mathrm{mg} / \mathrm{mL})\end{array}$ & $\begin{array}{c}\text { Conversion } \\
\qquad(\%)\end{array}$ & $\begin{array}{l}\text { Yield }^{d} \\
(\%)\end{array}$ & $\begin{array}{c}3 \mathbf{a} / \mathbf{4} \mathbf{a}^{d} \\
\text { ratio }\end{array}$ \\
\hline 1 & 5 & 1.2 & 10 & - & 14 & 10 & $71 / 29$ \\
\hline 2 & 5 & 1.2 & 10 & 10 & 100 & 90 & $90 / 10$ \\
\hline 3 & 5 & 1.2 & 10 & 15 & 85 & 74 & $87 / 13$ \\
\hline 4 & 5 & 1.2 & 10 & 5 & 87 & 75 & $86 / 14$ \\
\hline 5 & $20^{e}$ & 1.2 & 10 & 10 & 100 & 92 & $92 / 8$ \\
\hline 6 & 5 & 1.5 & 10 & 10 & 92 & 80 & $87 / 13$ \\
\hline 7 & 5 & 1 & 10 & 10 & 77 & 66 & $85 / 15$ \\
\hline 8 & 5 & 1.2 & 20 & 10 & 100 & 84 & $84 / 16$ \\
\hline 9 & 5 & 1.2 & 5 & 10 & 57 & 50 & $87 / 13$ \\
\hline 10 & 5 & 1.2 & $10^{e}$ & 10 & 40 & 32 & $80 / 20$ \\
\hline 11 & 5 & 1.2 & $10^{f}$ & 10 & 50 & 42 & $84 / 16$ \\
\hline 12 & 5 & 1.2 & $10^{g}$ & 10 & 55 & 48 & $87 / 13$ \\
\hline 13 & 5 & 1.2 & 10 & $10^{h}$ & 81 & 70 & $86 / 14$ \\
\hline 14 & 5 & 1.2 & 10 & $40^{i}$ & 73 & 56 & $76 / 24$ \\
\hline 15 & 5 & - & 10 & 10 & 0 & 0 & 0 \\
\hline $16^{j}$ & 5 & 1.2 & 10 & 10 & 0 & 0 & 0 \\
\hline 17 & 5 & 1.2 & 10 & 22 in $2 \mathrm{~mL}$ & 55 & 51 & $93 / 7$ \\
\hline 18 & 5 & 1.2 & 10 & 45 in $4 \mathrm{~mL}$ & 24 & 22 & $92 / 8$ \\
\hline $19^{k}$ & 5 & 1.2 & 10 & 10 & 73 & 23 & $32 / 68$ \\
\hline $20^{\prime}$ & 5 & 1.2 & 10 & 10 & 63 & 17 & $27 / 73$ \\
\hline $21^{m}$ & 5 & 1.2 & 10 & 10 & 10 & 6 & $73 / 27$ \\
\hline $22^{n}$ & 5 & 1.2 & 10 & 10 & 46 & 12 & $27 / 73$ \\
\hline $23^{\circ}$ & 5 & 1.2 & 10 & 10 & 18 & 6 & $35 / 65$ \\
\hline $24^{p}$ & 5 & 1.2 & 10 & 10 & 12 & 0 & 0 \\
\hline $25^{q}$ & 5 & 1.2 & 10 & 10 & 34 & 20 & $61 / 39$ \\
\hline $26^{r}$ & 5 & 1.2 & 10 & 10 & 94 & 80 & $86 / 14$ \\
\hline $27^{s}$ & 5 & 1.2 & 10 & 10 & 11 & 7 & $64 / 36$ \\
\hline $28^{t}$ & 5 & 1.2 & 10 & 10 & 8 & 3 & $42 / 58$ \\
\hline $29 u$ & 5 & 1.2 & 10 & 10 & 57 & 15 & $27 / 63$ \\
\hline
\end{tabular}




$\begin{array}{cccccccc}30^{v} & 5 & 1.2 & 10 & 10 & 90 & 79 & 88 / 12 \\ 31^{w} & 5 & 1.2 & 10 & 10 & 67 & 60 & 89 / 11 \\ 32 & 5 & \mathrm{Et}_{3} \mathrm{~N} 1.2 & 10 & 10 & 63 & 57 & 91 / 9 \\ 33 & 5 & \mathrm{DIPA} 1.2 & 10 & 10 & 35 & 31 & 89 / 11 \\ 34 & 5 & \mathrm{DBU} 1.2 & 10 & 10 & 93 & 74 & 80 / 20 \\ 35 & 5 & \mathrm{DABCO}^{2} 1.2 & 10 & 10 & 7 & 5 & 73 / 27 \\ 36 & 5 & \mathrm{~K}_{2} \mathrm{CO}_{3} 1.2 & 10 & 10 & 26 & 20 & 79 / 21 \\ 37 & 5 & \mathrm{Et}_{3} \mathrm{~N} 1.2 & 10 & 10 & 63 & 57 & 91 / 9 \\ 38^{\mathrm{x}} & 5 & 1.2 & 10 & - & 37 & 33 & 90 / 10\end{array}$

a 1a (7.2 mg, $0.05 \mathrm{mmol})$ with $\mathbf{G} 1$ in $1 \mathrm{~mL}$ of anhydrous $A C N$; irradiation with cold white-light $(410-700 \mathrm{~nm})$ LEDs at $23^{\circ} \mathrm{C}$ for 4 hours unless otherwise indicated. ${ }^{b}$ DCA as photocatalyst unless otherwise indicated. ${ }^{c} \mathbf{G}$ : gelator. $\mathrm{H}-$ bonding and van der Waals forces trigger the self-assembly process of gelators in organic solvent, affording tangled fibrillar nanostructures over a wide concentration range. ${ }^{*}$ Conversions, yields and ratios were calculated from quantitative GC analysis vs. internal 1-dodecanonitrile. ${ }^{e} 2$ hours of irradiation. ${ }^{f}$ $\mathrm{N}, \mathrm{N}$-bis(2,6-diisopropylphenyl)perylene-3,4,9,10-bis(dicarboximide) (PDI).

Rhodamine 6G (Rh6G). ${ }^{g}$ Sulforhodamine B (SRhB). ${ }^{h}$ G2. ${ }^{i}$ G3. ${ }^{j}$ Using Blue $(420 \mathrm{~nm})$ lamps or Green (520 nm) LEDs. ${ }^{\mathrm{D} D M F}$, 'DMA, ${ }^{\mathrm{m} A c O E t,}{ }^{n} \mathrm{EtOH}$, oDioxane, ${ }^{p}$ Toluene, ${ }^{\circ} \mathrm{DCM}$, rAcetone, ${ }^{s}$ Ether, ${ }^{t}$ Hexane, uDMSO, ${ }^{v} \mathrm{ACN}$, ${ }^{w} \mathrm{ACN} / \mathrm{H}_{2} \mathrm{O}(9 / 1, \mathrm{v} / \mathrm{v})$, ${ }^{\mathrm{x}}$ under anaerobic conditions.

\footnotetext{
* (a) Li, Y.; Wang, T.; Liu, M. Gelating-induced supramolecular chirality of achiral porphyrins: chiroptical switch between achiral molecules and chiral assemblies. Soft Matter 2007, 3, 1312-1317. (b) Hanabusa, K.; Yamada, M.; Kimura, M.; Shirai, H. Prominent Gelation and Chiral Aggregation of Alkylamides Derived from trans- 1,2-Diaminocyclohexane. Angew. Chem., Int. Ed. Engl. 1996, 35, 1949-1951. (c) Watase, M.; Nakatani, Y.; Itagaki, H. On the Origin of the Formation and Stability of Physical Gels of Di-Obenzylidene-D-sorbitol. J. Phys. Chem. B 1999, 103, 13, 2366-2373
} 


\section{Cyclic Voltammetry}

The redox potentials were measured by cyclic voltammetry with an Solartron 1284 potentiostat. All measurements were made in deaerated acetonitrile containing tetrabutylammonium tetrafluoroborate $(0.1 \mathrm{M})$ as supporting electrolyte, a glassy carbon as working electrode, a platinum wire as counter electrode, a silver wire as pseudo reference and ferrocene $(0.01 \mathrm{M})$ as internal standard. The scan rate was $100 \mathrm{mV} \cdot \mathrm{s}^{-1}$. Potentials are reported with respect to the saturated calomel electrode (SCE) as reference.
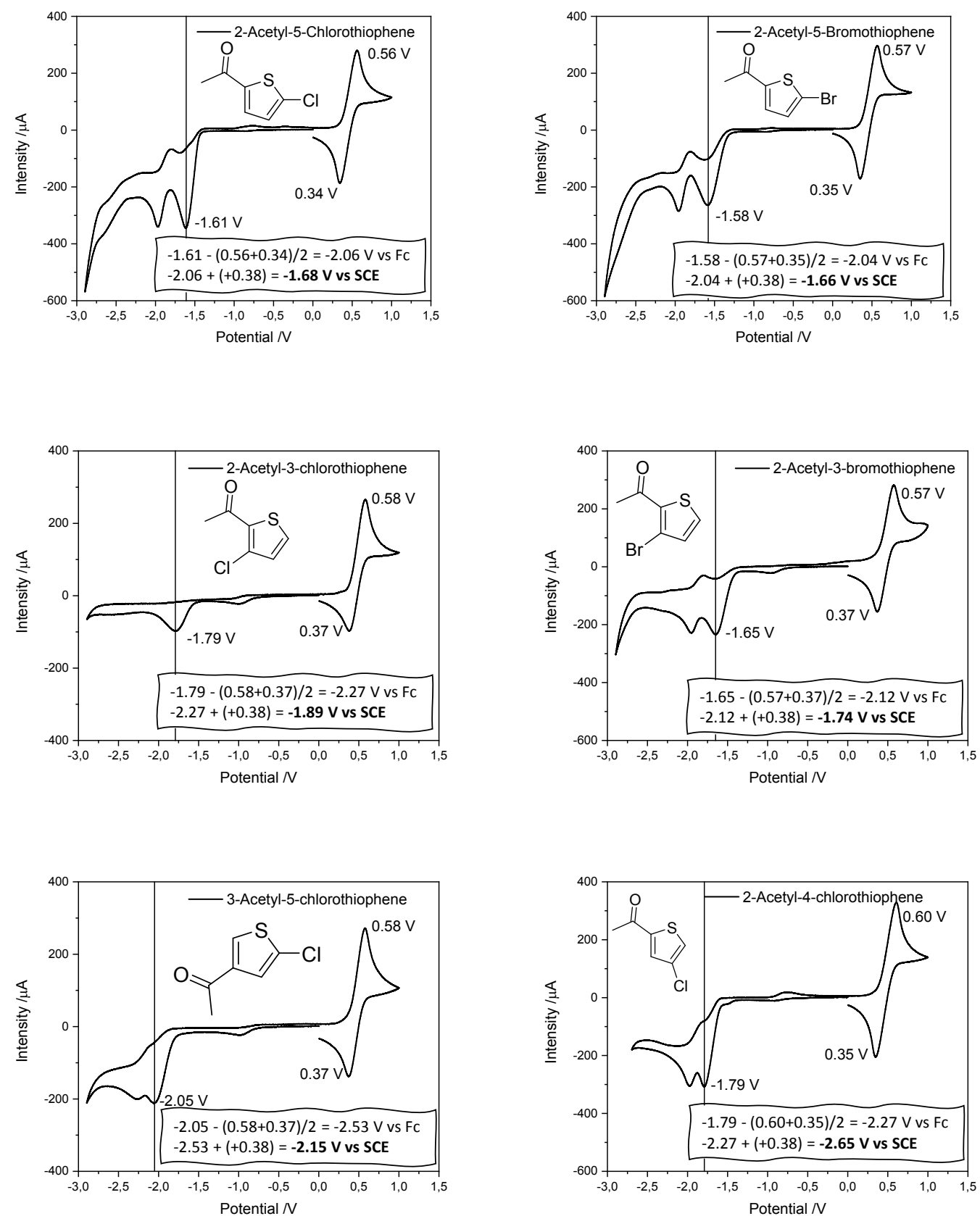

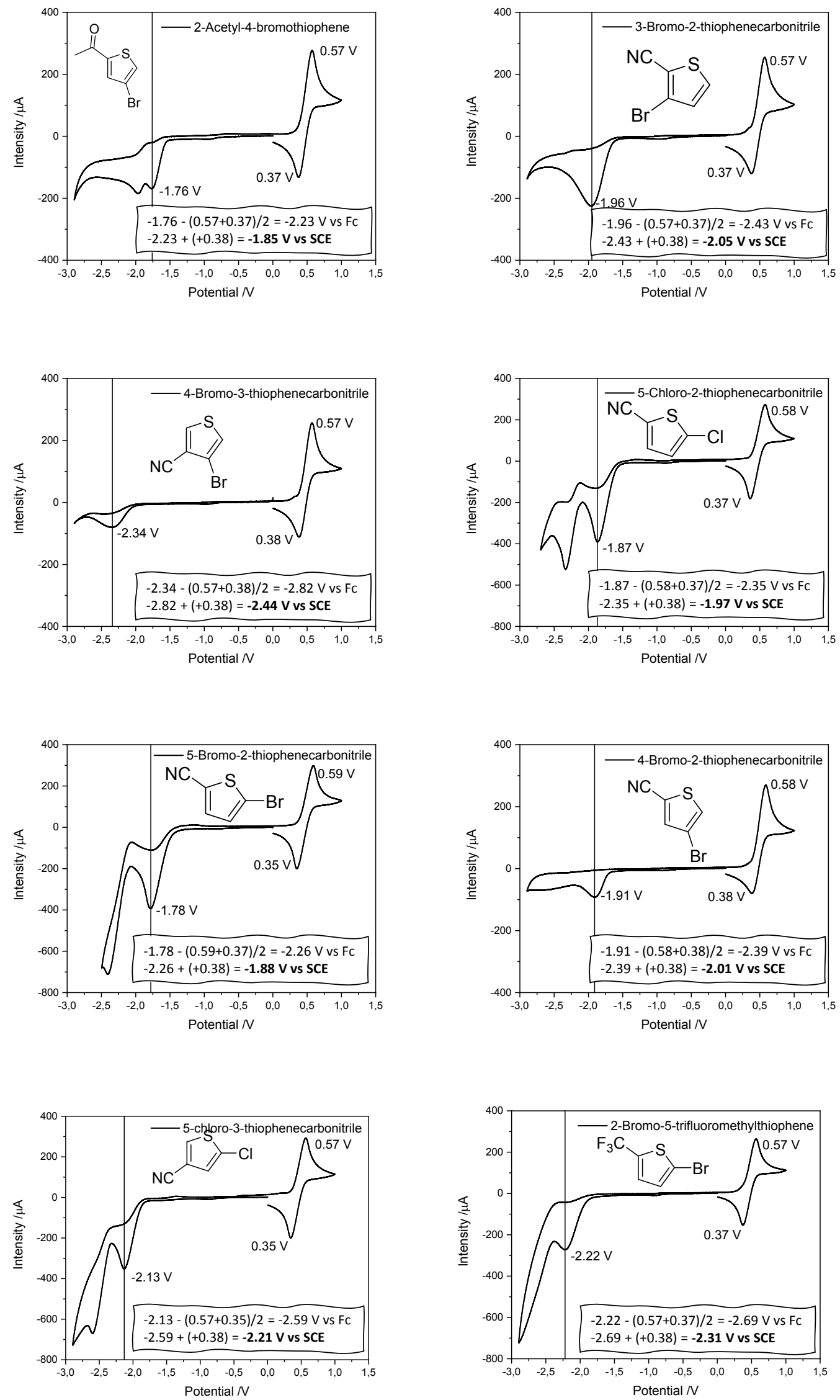

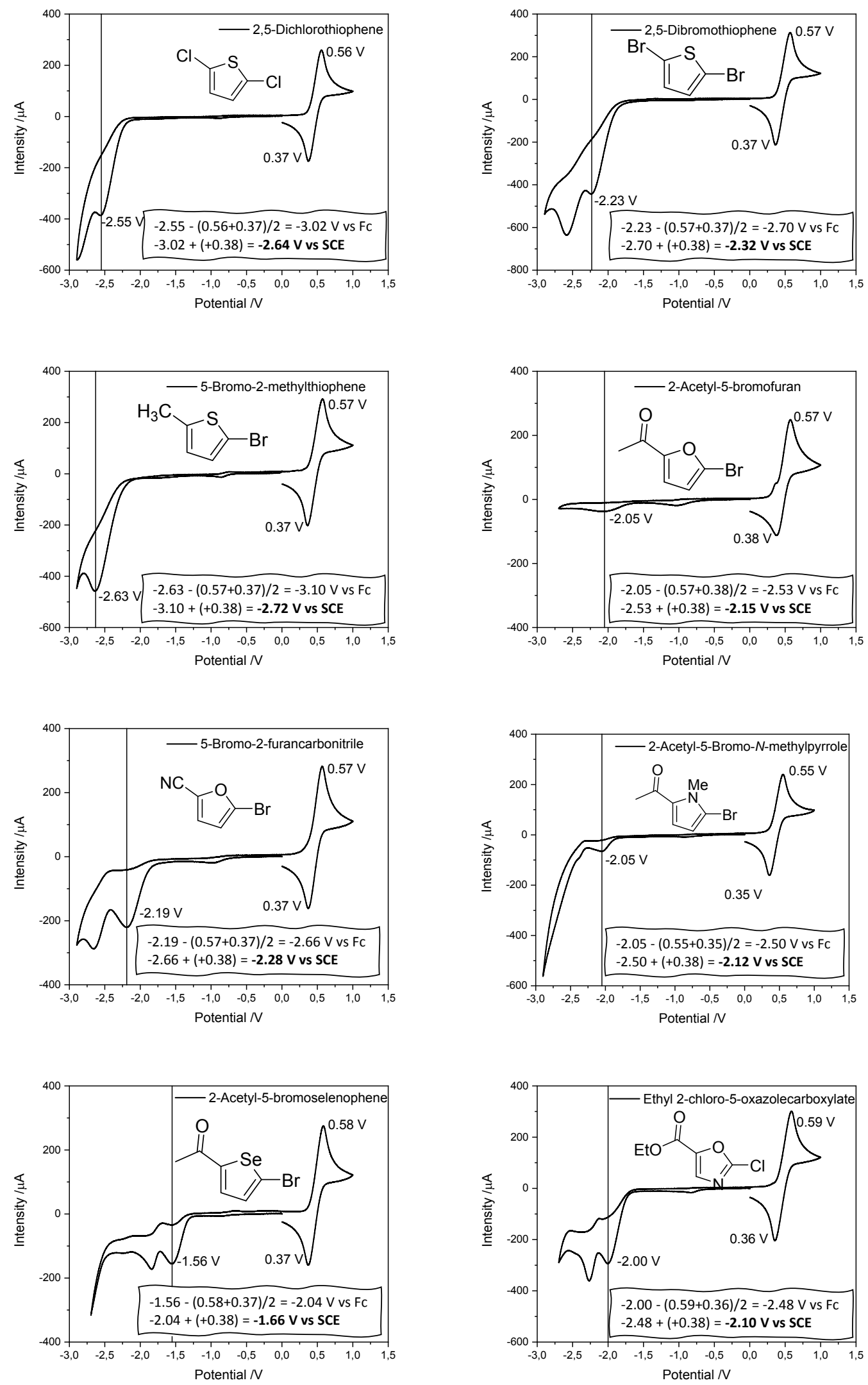

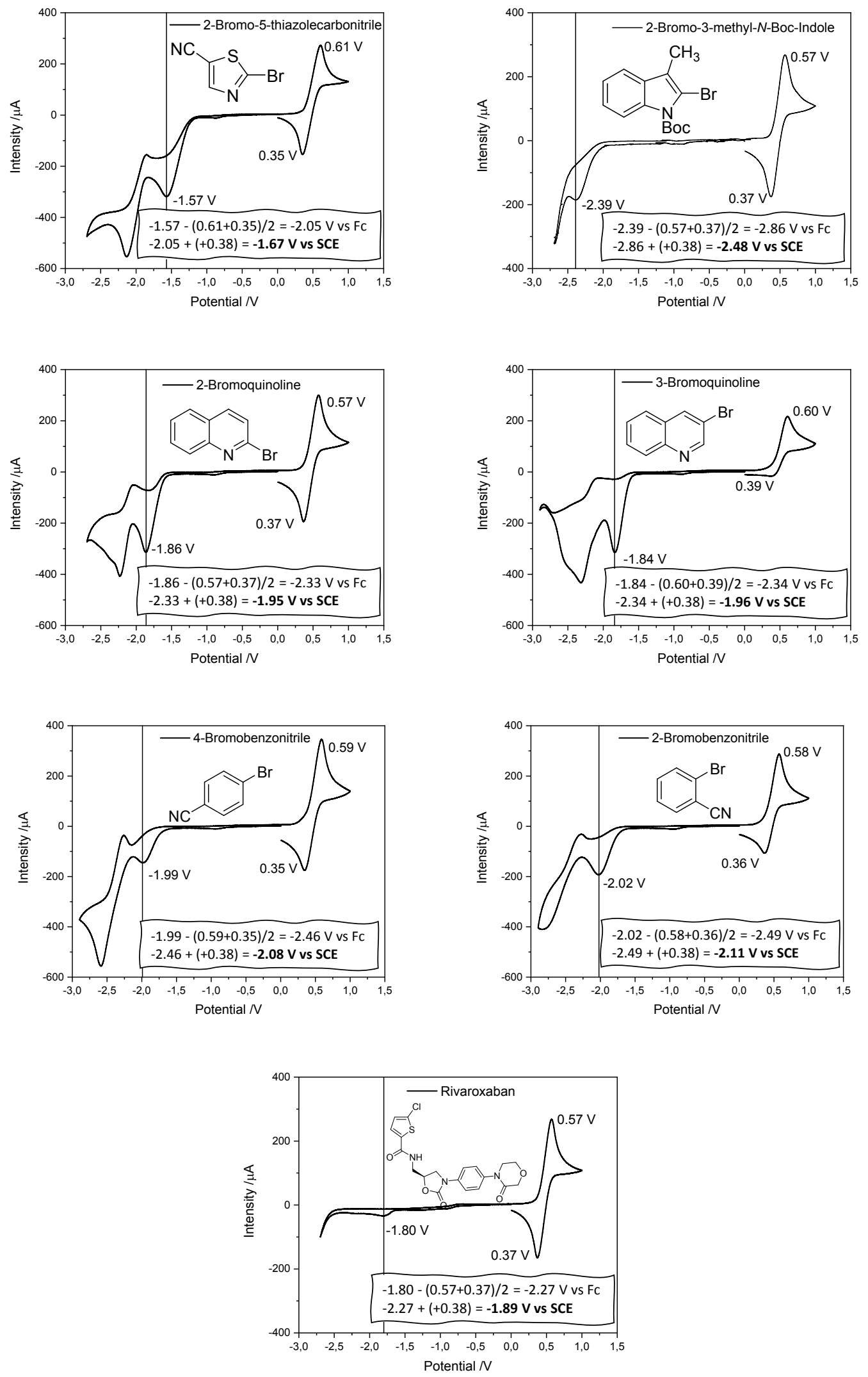

Figure S1. Cyclic voltammetry spectra of the corresponding (hetero)arene halides. 


\section{Kinetic studies}
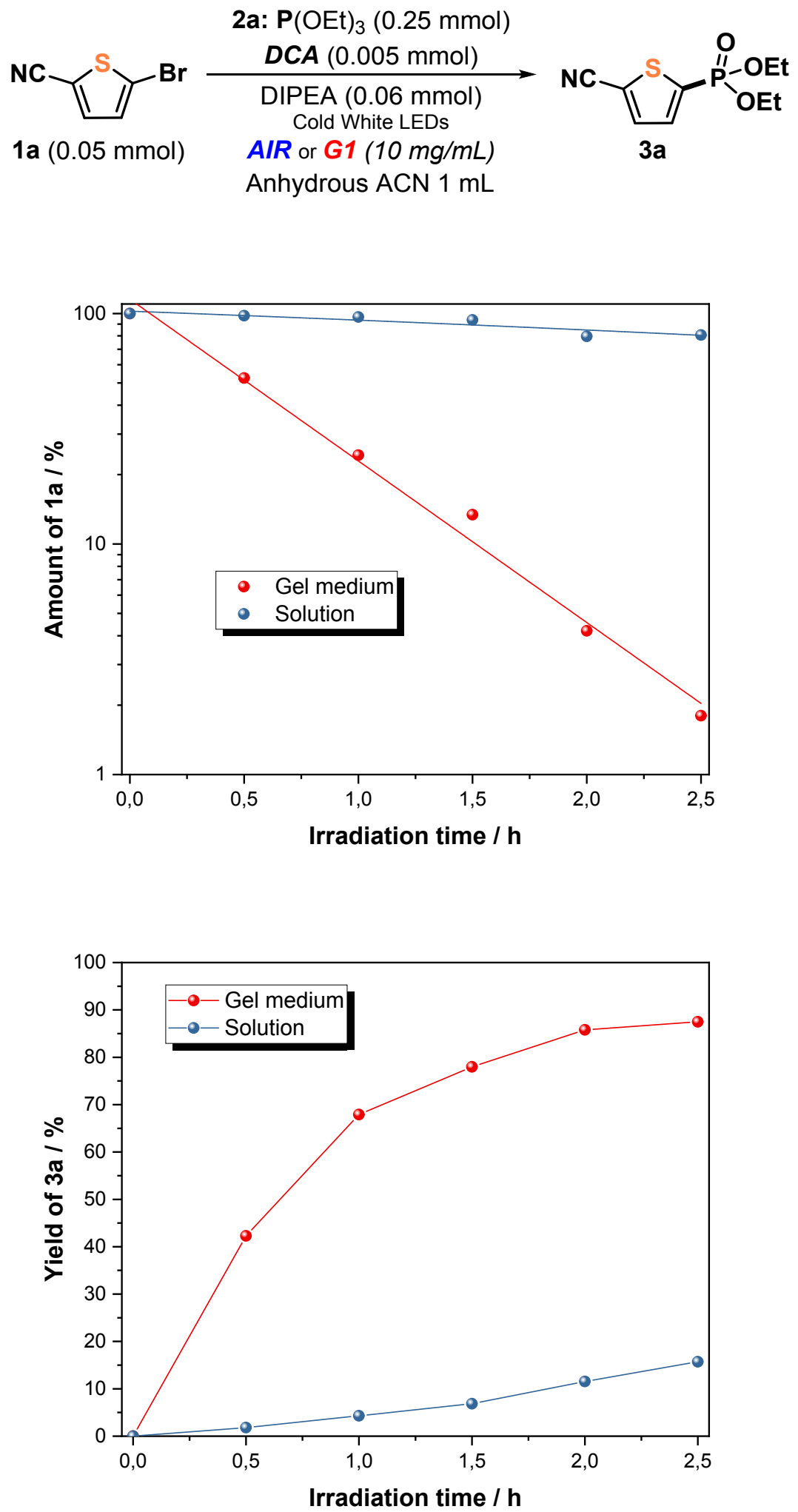

Figure S2. Top: Relationship between conversion of 1a versus irradiation time. Bottom: Relationship between production of $\mathbf{3 a}$ versus irradiation time. 


\section{Frozen model reaction procedure}

Scheme S1. Frozen reaction conditions for the phosphorylation of 1a.

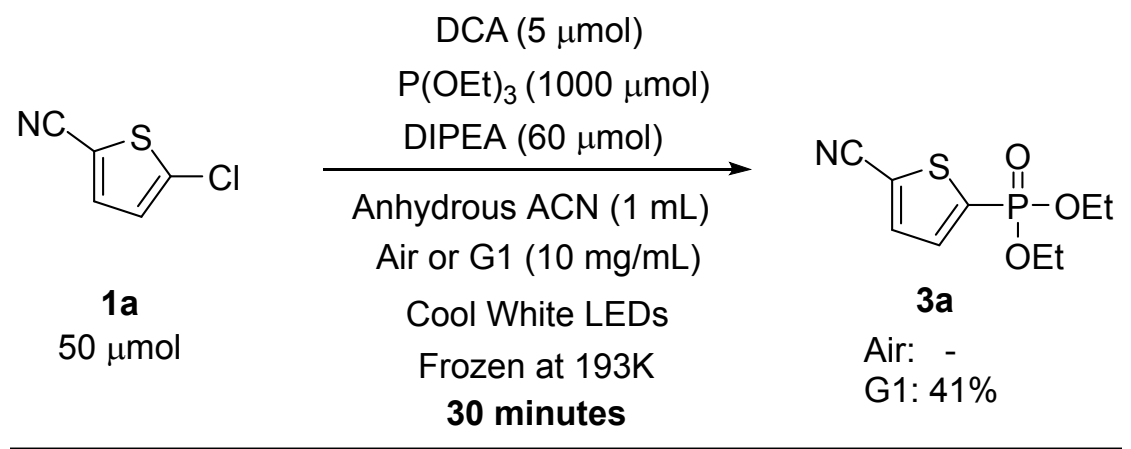

A vial $(10 \mathrm{~mL})$ was charged with 9,10-anthracenedicarbonitrile $(1.2 \mathrm{mg}, 5 \mu \mathrm{mol}, 10 \mathrm{~mol} \%$ and the correspondent gelator (G1, $10 \mathrm{mg})$. Anhydrous acetonitrile $(1.0 \mathrm{~mL})$ was poured and 5-chloro-2-thiophenecarbonitrile $(5.4 \mu \mathrm{L}, 50 \mu \mathrm{mol}, 1.0$ equiv.) and triethylphosphite (180 $\mu \mathrm{L}, 1000 \mu \mathrm{mol}, 20.0$ equiv.) were added. Then, DIPEA $(10.5 \mu \mathrm{L}, 60 \mu \mathrm{mol}, 1.2$ equiv.) and dodecanenitrile $(12.0 \mu \mathrm{L}, 50 \mu \mathrm{mol}, 1.0$ equiv.) were added with $25 \mu \mathrm{L}$ Hamilton syringe. Quickly, the vial was sealed with a septum. It was heated to $150{ }^{\circ} \mathrm{C}$ with heatgun for 1.5 minutes with manual stirring until complete clear solution. The vial cooled to room temperature until gel formation was observed. The vial was frozen at $193 \mathrm{~K}$ for 2 hours. Later, the frozen vials were irradiated with an external LED through the plain bottom side of the vial at $23{ }^{\circ} \mathrm{C}$ for 30 minutes. Finally, the reactions were monitored by GC-FID analysis. The gel was broken with ethyl acetate $(1 \mathrm{~mL})$, and it was clean with brine (2 $\mathrm{mL}$ ). The organic phase was dried over anhydrous sodium sulfate, filtered from the drying agent, and concentrated in vacuo. 


\section{UV-vis absorption spectra and DCA fluorescence quenching}

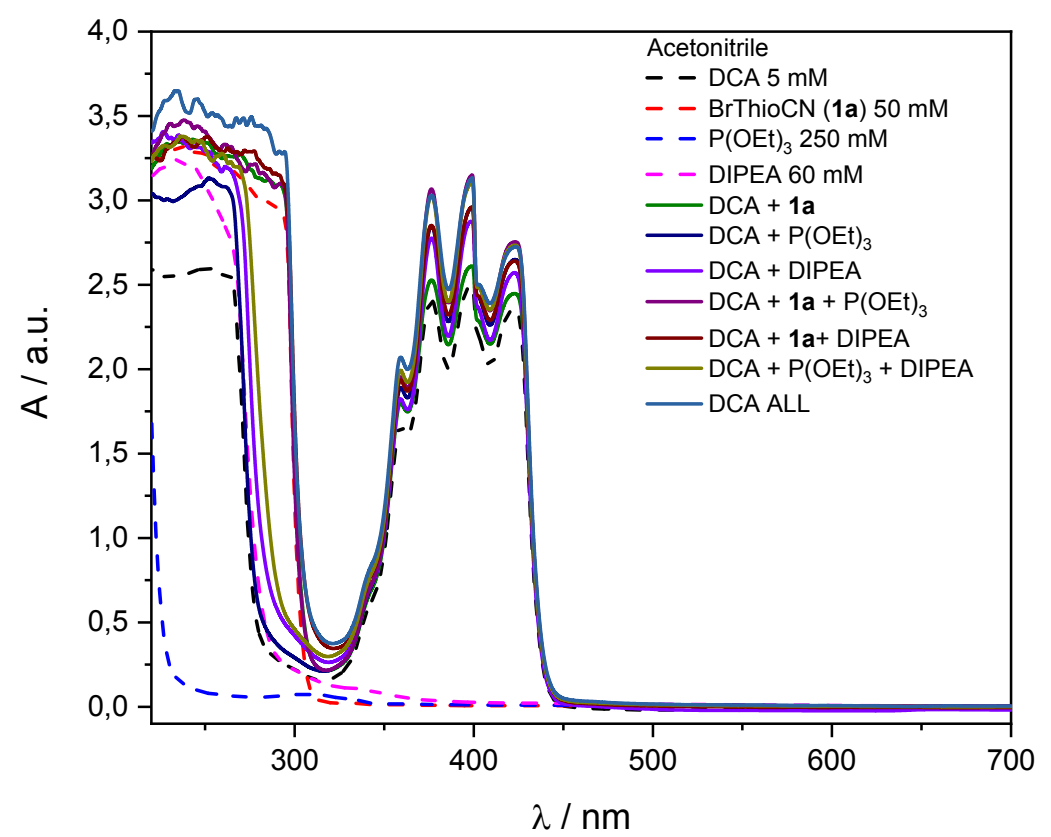

Figure S3. Absorption spectra of the corresponding photocatalyst and substrates in acetonitrile.

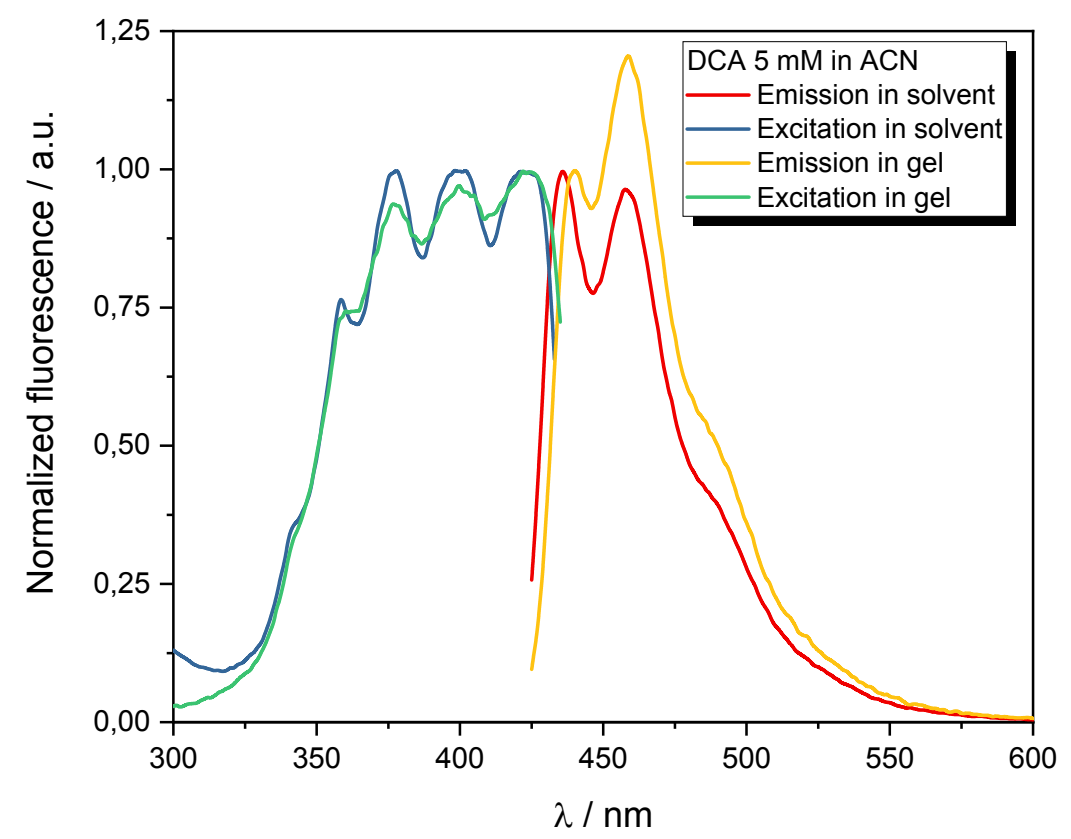

Figure S4. Emission and excitation spectra of DCA. 


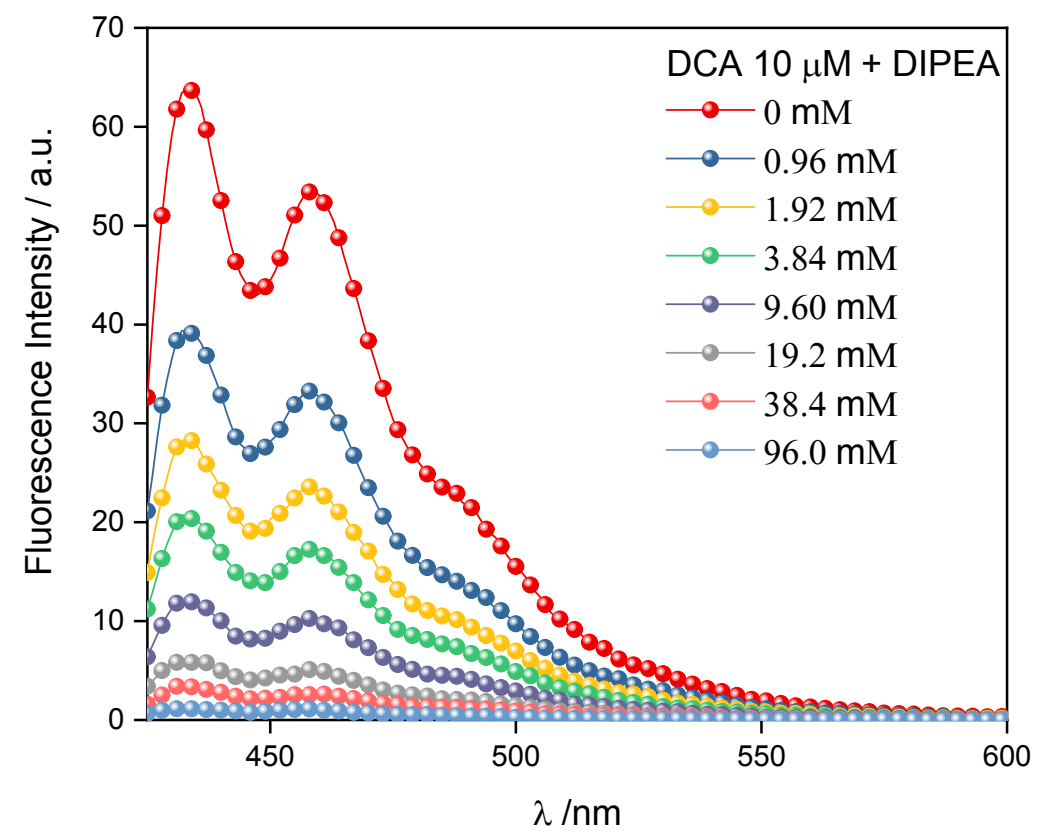

Figure S5. Emission spectra of DCA $(10 \mu \mathrm{M})$ in the presence of increasing amounts of DIPEA in acetonitrile under aerobic conditions.

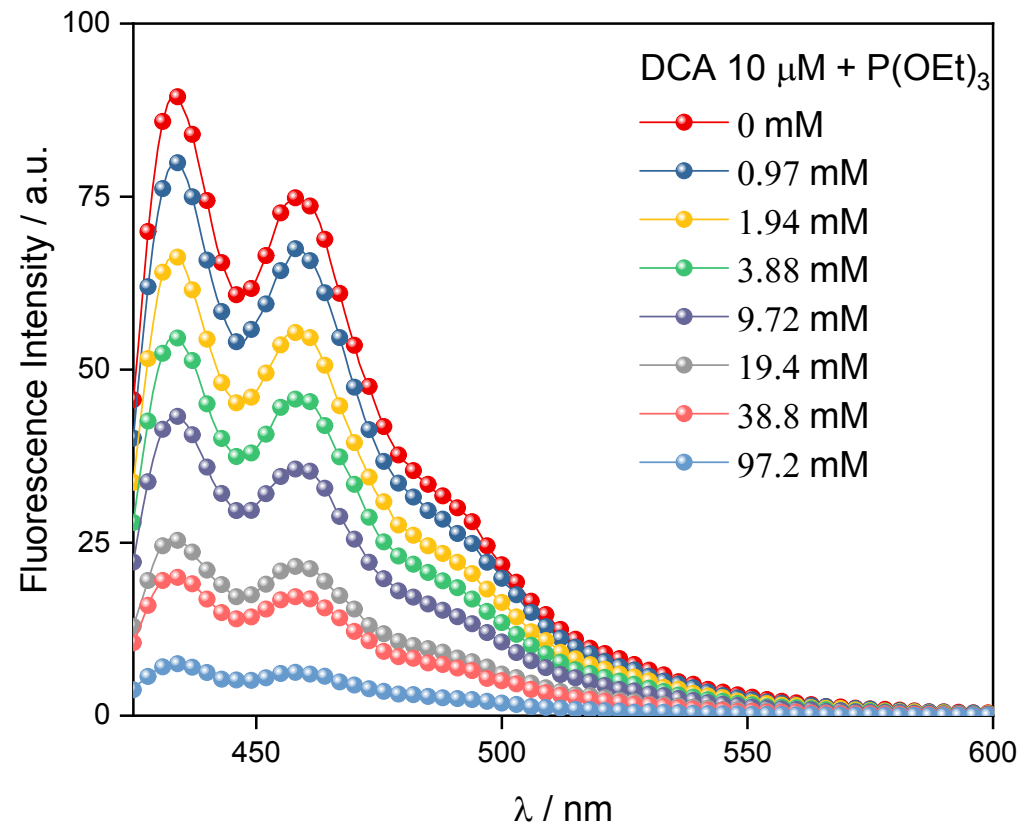

Figure S6. Emission spectra of DCA $(10 \mu \mathrm{M})$ in the presence of increasing amounts of triethyl phosphite in acetonitrile under aerobic conditions. 


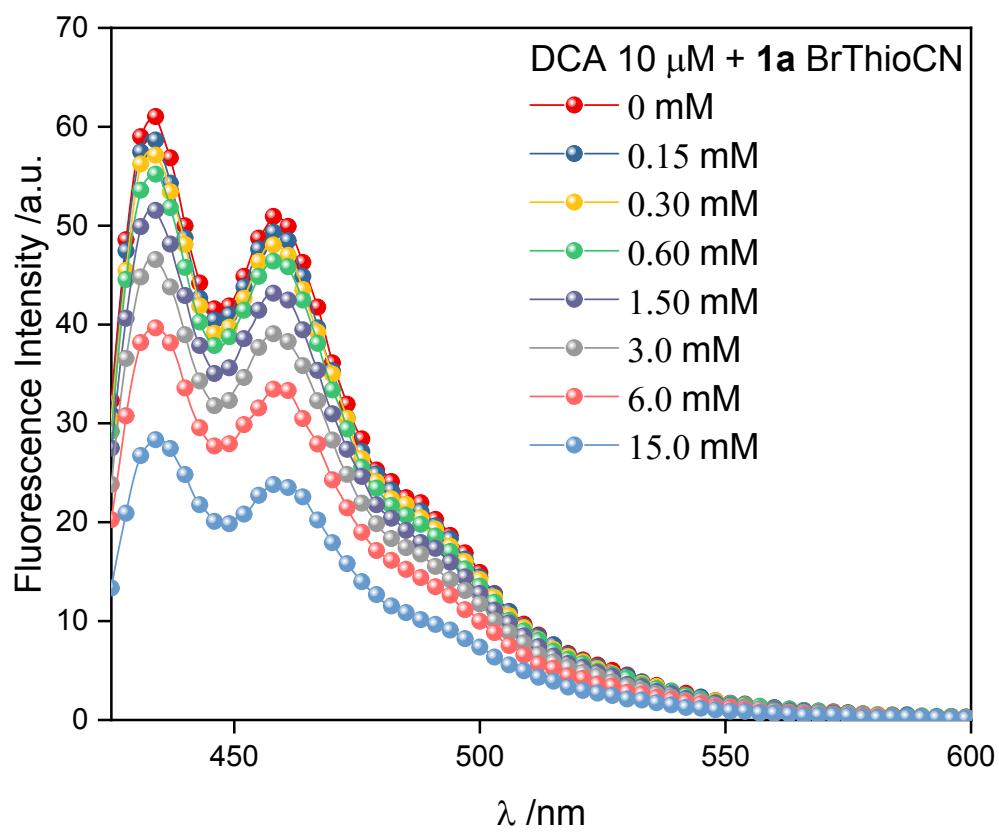

Figure S7. Emission spectra of DCA $(10 \mu \mathrm{M})$ in the presence of increasing amounts of 2-bromo-5-thiophenecarbonitrile in acetonitrile under aerobic conditions.

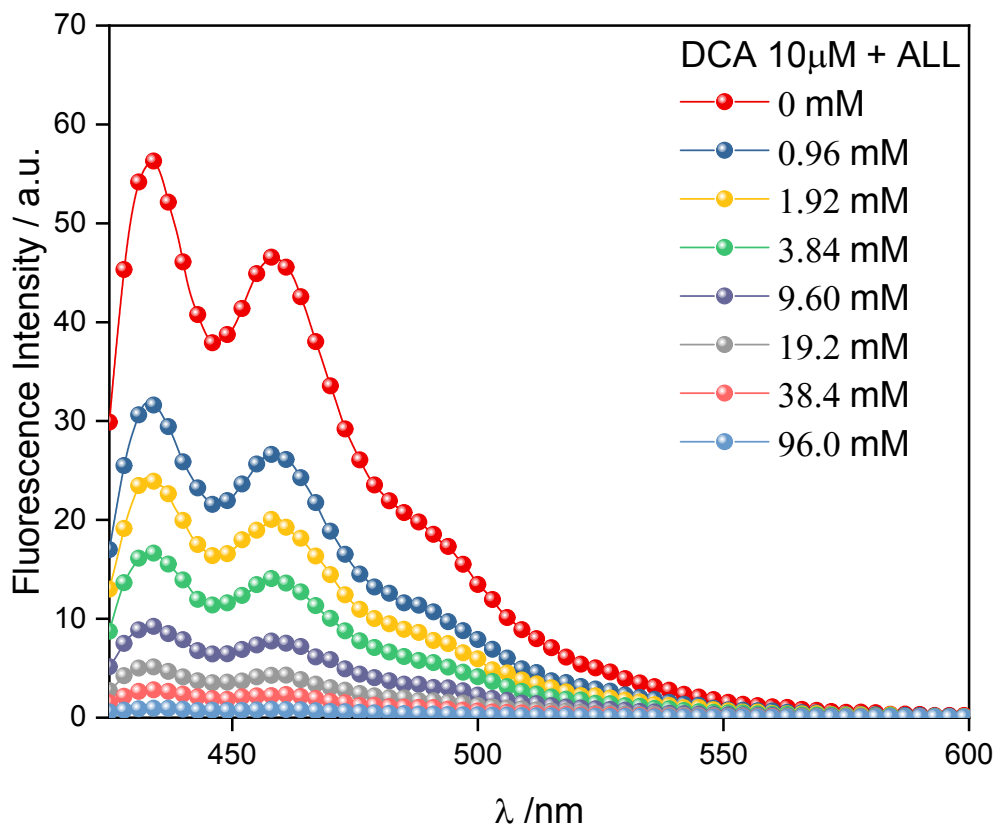

Figure S8. Emission spectra of DCA $(10 \mu \mathrm{M})$ in the presence of increasing amounts of DIPEA + triethyl phosphite + 2-bromo-5-thiophenecarbonitrile in acetonitrile under aerobic conditions. 


\section{Trapping reaction}

Scheme S2. Trapping reaction of 1 a by diphenyl sulfide.

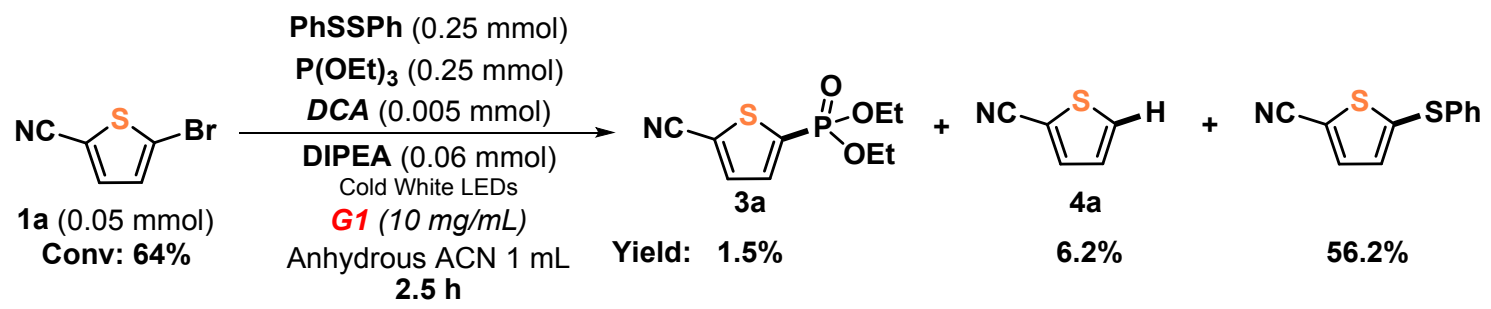

A vial $(10 \mathrm{~mL})$ was charged with 9,10-anthracenedicarbonitrile $(1.2 \mathrm{mg}, 5 \mu \mathrm{mol}, 10$ mol\%), diphenyl sulfide ( $47.5 \mathrm{mg}, 250 \mu \mathrm{mol}, 5.0$ equiv.) and the correspondent gelator (G1, $10 \mathrm{mg})$. Anhydrous acetonitrile $(1.0 \mathrm{~mL})$ was poured and 5-bromo-2thiophenecarbonitrile $(5.7 \mu \mathrm{L}, 50 \mu \mathrm{mol}, 1.0$ equiv.) and triethylphosphite (45 $\mu \mathrm{L}, 250$ $\mu \mathrm{mol}, 5.0$ equiv.) were added. Then, DIPEA (10.5 $\mu \mathrm{L}, 60 \mu \mathrm{mol}, 1.2$ equiv.) and dodecanenitrile (12.0 $\mu \mathrm{L}, 50 \mu \mathrm{mol}, 1.0$ equiv.) were added with $25 \mu \mathrm{L}$ Hamilton syringe. Quickly, the vial was sealed with a septum. It was heated to $150^{\circ} \mathrm{C}$ with heatgun for 1.5 minutes with manual stirring until complete clear solution. The vial cooled to room temperature until gel formation was observed. The reaction was irradiated with an external LED through the plain bottom side of the vial at $23^{\circ} \mathrm{C}$ for 2.5 hours. Then, brine $(2 \mathrm{~mL})$ was added, and the aqueous phase was extracted with ethyl acetate $(1 \mathrm{~mL})$. The reaction was monitored by GC-FID analysis obtaining. The organic phase was dried over anhydrous sodium sulfate, filtered from the drying agent, and concentrated in vacuo. The crude was purified via flash column chromatography using a hexane/ethyl acetate mixture as the mobile phase. 


\section{Sunlight irradiation and $1 \mathrm{mmol}$ scale}

Sunlight irradiation

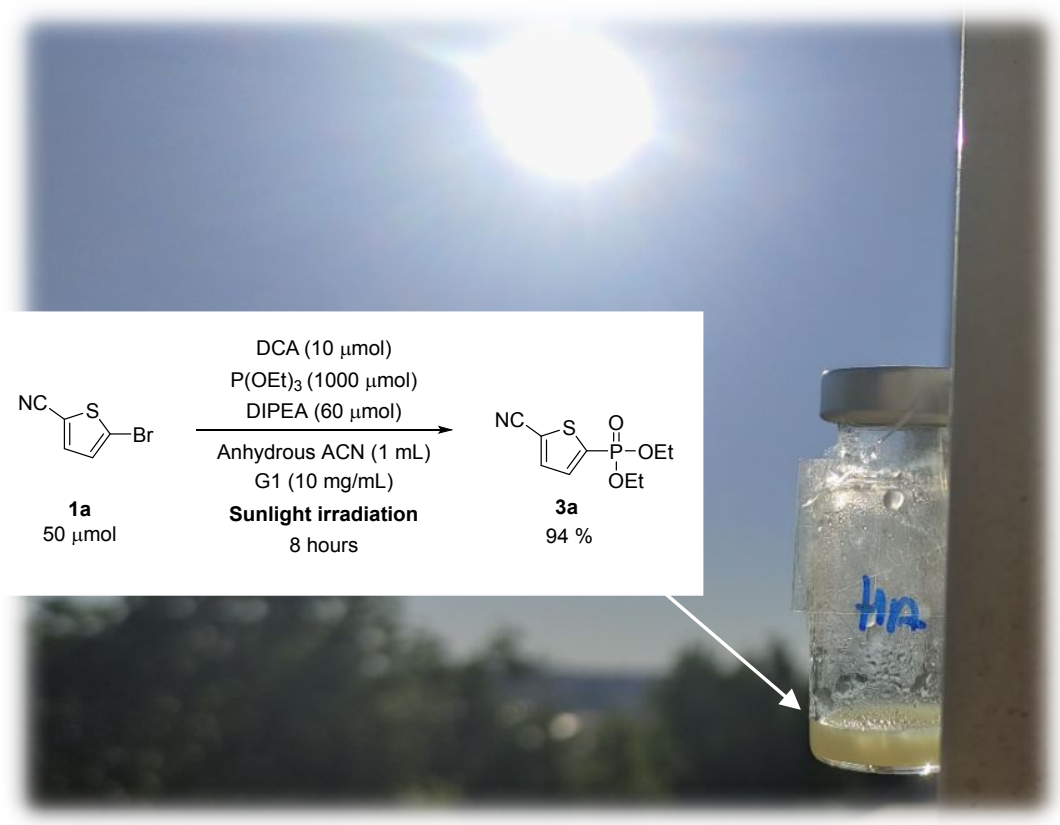

Figure S9. Photograph of the reaction performed by external sunlight irradiation.

The compound 3a was prepared according to the general procedure using 9-10dicyanoanthracene $(2.4 \mathrm{mg}, 10 \mu \mathrm{mol}, 20 \mathrm{~mol} \%)$ as photocatalyst, 5-bromo-2thiophenecarbonitrile $(5.7 \mu \mathrm{L}, 50 \mu \mathrm{mol}, 1.0$ equiv. $)$, triethylphosphite $(180 \mu \mathrm{L}, 1000 \mu \mathrm{mol}$, 20.0 equiv.), dodecanenitrile (12.0 $\mu \mathrm{L}, 50 \mu \mathrm{mol}, 1.0$ equiv.) as internal standard and DIPEA ( $10.5 \mu \mathrm{L}, 60 \mu \mathrm{mol}, 1.2$ equiv.) and $\mathrm{G} 1(10 \mathrm{mg} / \mathrm{mL})$. The gel mixture was irradiated with sunlight for 8 hours, obtaining 94\% product yield according to GC-FID analysis.

Location: Camí de Vera S/N, Chemistry Department, Universitat Politècnica de València (UPV), Valencia, Spain (coordinate: $39.482917,-0.341642$ ), temperature: $16-23^{\circ} \mathrm{C}$, from 9:00 to $17: 00$. Date: $24 / 05 / 2021$ 
$1 \mathrm{mmol}$ scale

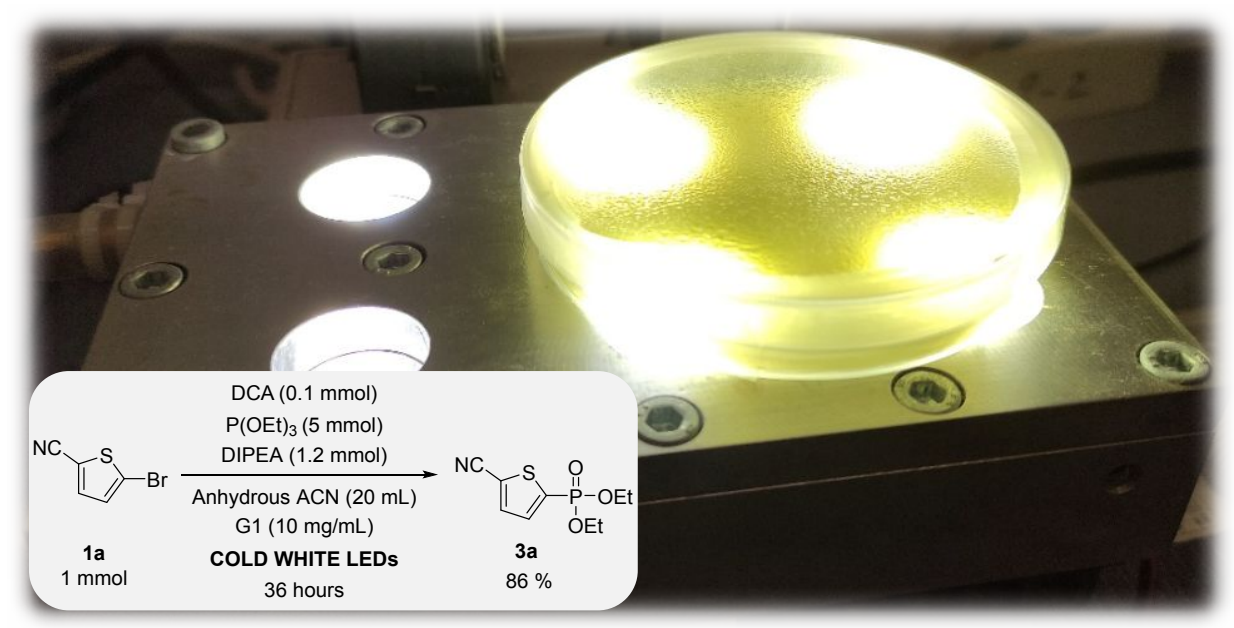

Figure S10. Photograph of the reaction performed by white light LEDs for the scaling-up conditions.

To scale-up the procedure (from $0.05 \mathrm{mmol}$ to $1 \mathrm{mmol}$ ), we performed the reaction following the general procedure inside a covered petri dish using 9-10dicyanoanthracene (24 mg, $0.1 \mathrm{mmol}, 10 \mathrm{~mol} \%$ ) as photocatalyst, 5-bromo-2thiophenecarbonitrile $(114 \mu \mathrm{L}, 1 \mathrm{mmol}, 1.0$ equiv.), triethylphosphite $(900 \mu \mathrm{L}, 5 \mathrm{mmol}$, 5.0 equiv.), dodecanenitrile ( $240 \mu \mathrm{L}, 1 \mathrm{mmol}, 1.0$ equiv.) as internal standard and DIPEA (209 $\mu \mathrm{L}, 1.2 \mathrm{mmol}, 1.2$ equiv.) and $\mathrm{G} 1(200 \mathrm{mg} / \mathrm{mL})$ in $20 \mathrm{~mL}$ of anhydrous acetonitrile. The reaction mixture was irradiated for 36 hours with cold white LEDs. Then, brine (30 $\mathrm{mL}$ ) was added, and the aqueous phase was extracted with ethyl acetate $(3 \times 30 \mathrm{~mL})$. The reaction was monitored by GC-FID analysis obtaining $86 \%$ product yield. The organic phase was dried over anhydrous sodium sulfate, filtered from the drying agent, and concentrated in vacuo. The crude was purified via flash column chromatography using a hexane/ethyl acetate mixture as the mobile phase obtaining $161.7 \mathrm{mg}, 66 \%$ isolated yield. 


\section{Oscillatory rheology}

Oscillatory rheology was performed with an AR 2000 Advanced rheometer (TA Instruments) equipped with a Julabo C cooling system. A $1000 \mu \mathrm{m}$ gap setting and a torque setting of 40,000 dynes $\mathrm{cm}^{-2}$ at $25^{\circ} \mathrm{C}$ were used for the measurements in a plainplate $(40 \mathrm{~mm}$, stainless steel).

The following experiments were performed using $2 \mathrm{~mL}$ total gel volume: a) Dynamic strain sweep (DSS): variation of G' and G" with strain (from 0.01 to $100 \%$ ); b) dynamic frequency sweep (DFS): variation of G' and G" with frequency (from 0.1 to $10 \mathrm{~Hz}$ ar $0.1 \%$ strain). 


\section{Field-emission scanning electron microscopy (FESEM)}

The equipment in operation in the UPV Microscopy Service is the ZEISS ULTRA 55 model, incorporating the following detectors:

-A Secondary Electron Detector (SE2), which provides an SEM topography image of the sample surface with a large depth of field.

- A Secondary Electron In-Lens Detector located inside the electron column, which works with low energy secondary electrons and provides images with a higher resolution.

- A Backscattered Electron Detector (AsB) which is sensitive to the variation of atomic number in the elements present in the sample; therefore, it is used to observe changes in the chemical composition of the specimen.

- A Backscattered Electron In-lens Detector (EsB), independent of the secondary In-lens detector, which provides a pure backscattered signal with no secondary electron contamination and very low acceleration potential.

- An X-Ray Dispersive Energy Detector, EDS, (Oxford Instruments) which receives Xrays from each surface point the electron beam passes over.

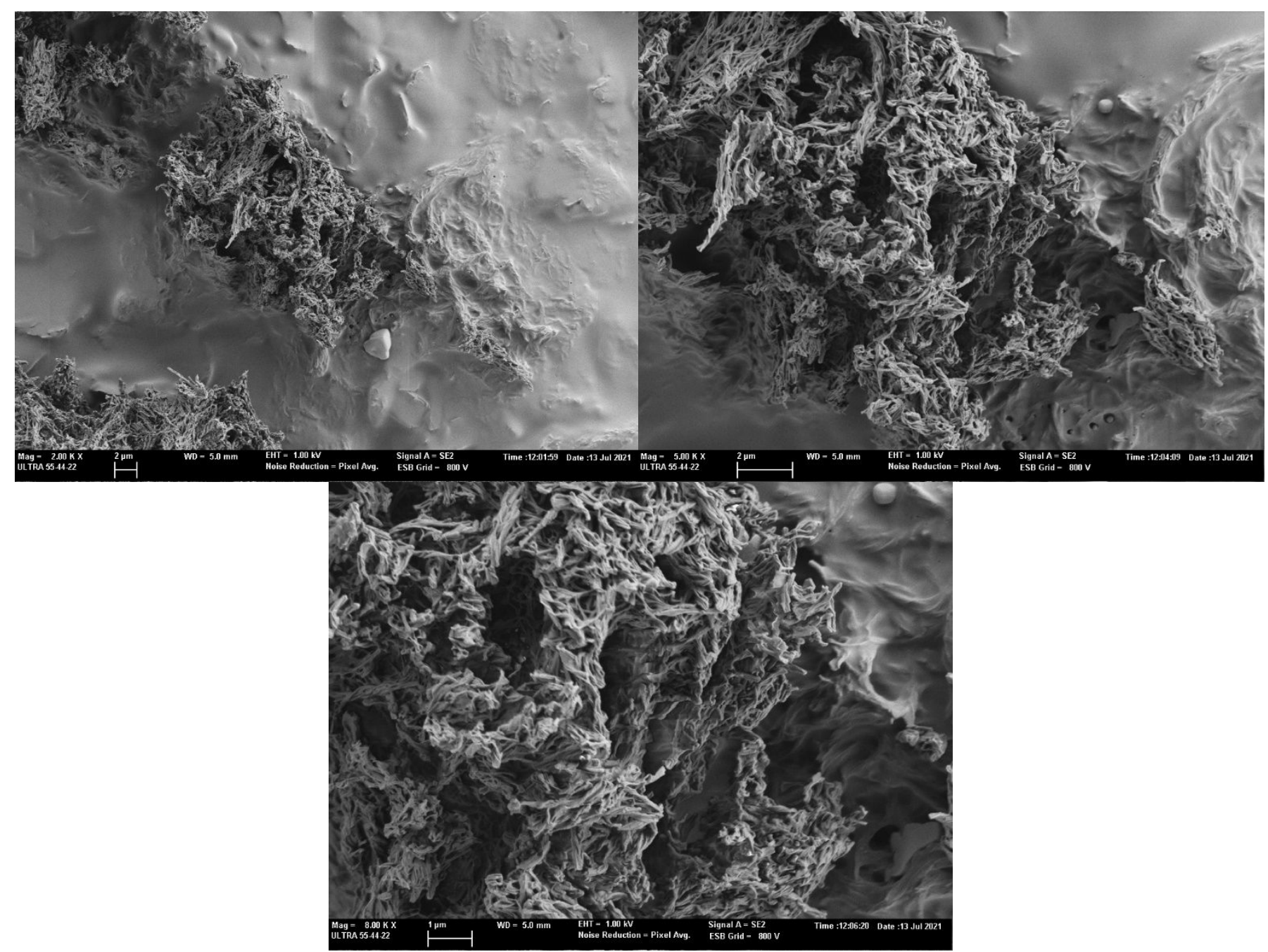

Figure S11. Representative images of FESEM of the unloaded G1 before irradiation. 

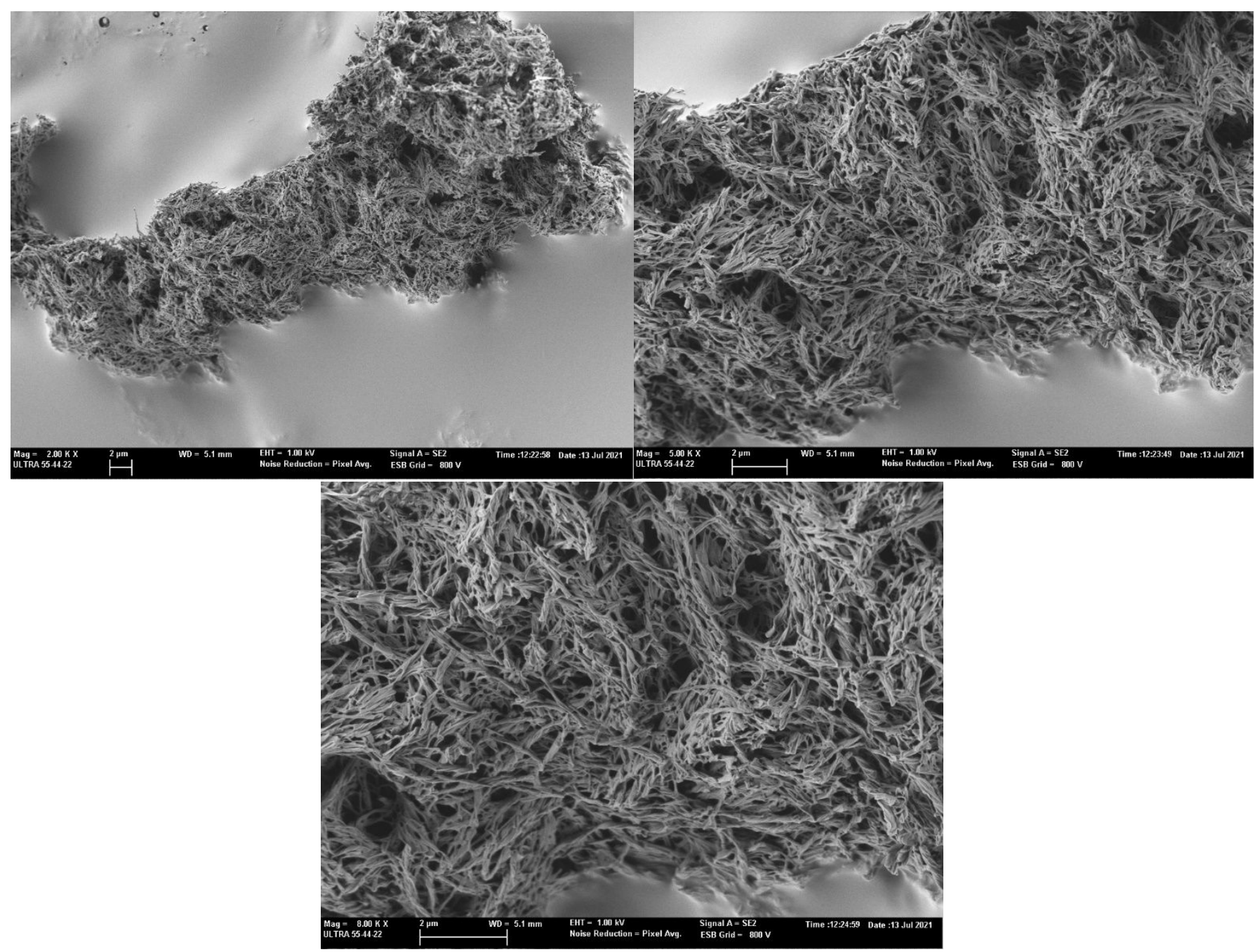

Figure S12. Representative images of FESEM of the unloaded G1 after 2.5 hours of irradiation. 


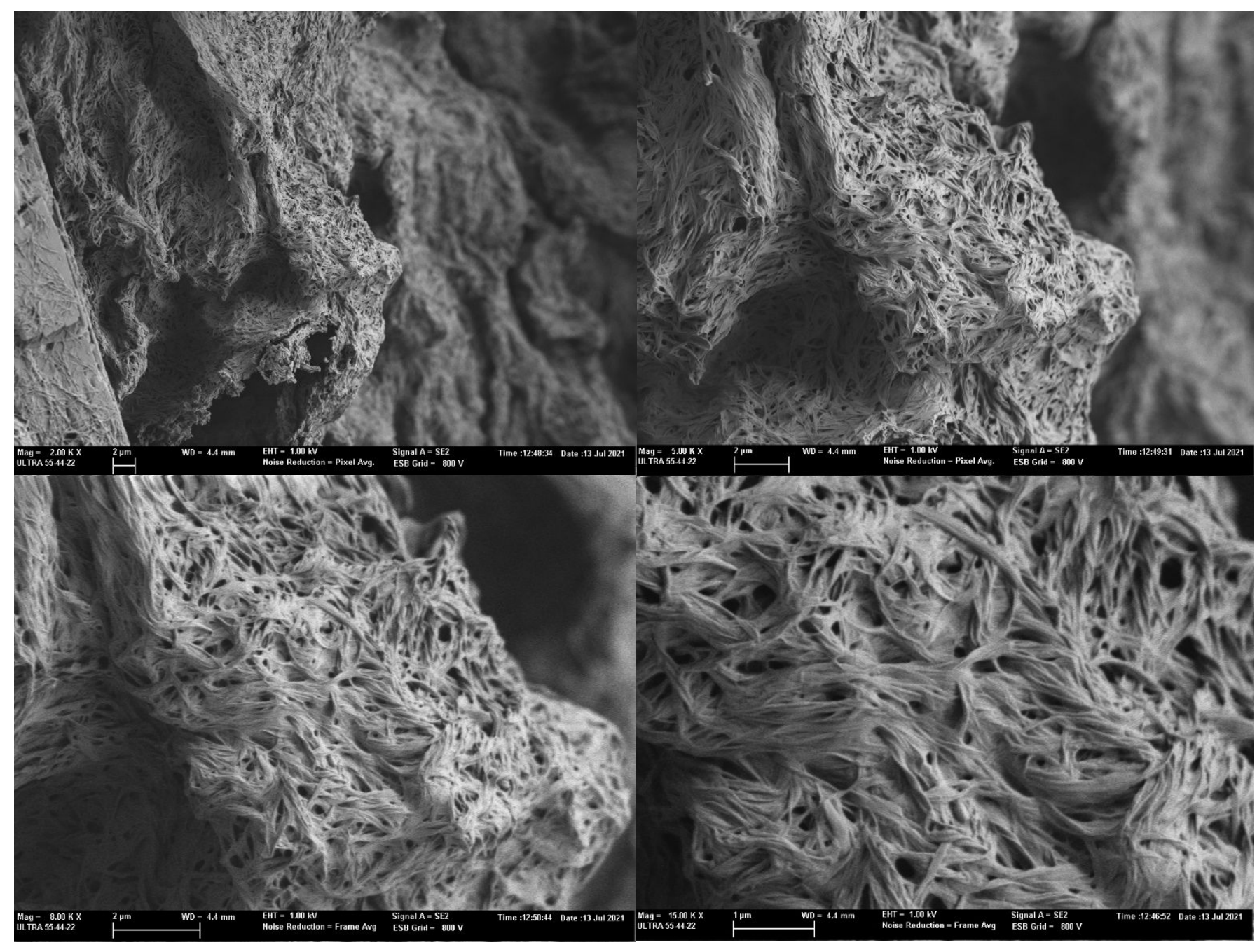

Figure S13. Representative images of FESEM of the loaded $\mathbf{G 1}$ before irradiation. 


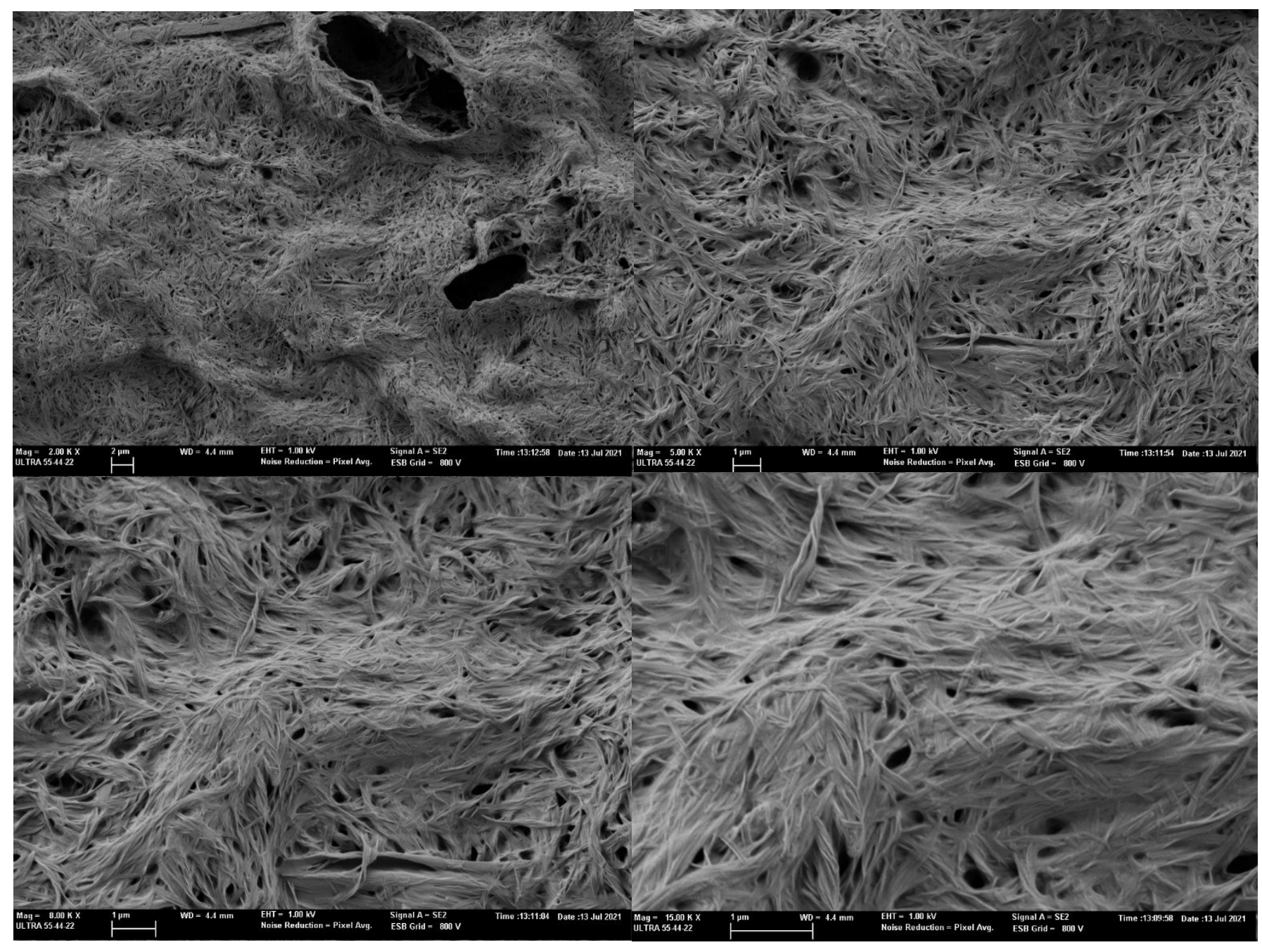

Figure S14. Representative images of FESEM of the loaded $\mathbf{G 1}$ after 2.5 hours of irradiation. 


\section{Characterization of compounds}

\section{THIOPHENES}

\section{3a. Diethyl (5-cyanothiophen-2-yl)phosphonate}<smiles>CCOP(=O)(OCC)c1ccc(C#N)s1</smiles>

The compound was prepared according to the general procedure using 9-10-dicyanoanthracene (1.2 mg, $5 \mu \mathrm{mol}, 0.1$ equiv.), 5bromo-2-thiophenecarbonitrile $(5.7 \mu \mathrm{L}, 50 \mu \mathrm{mol}, 1.0$ equiv.), triethylphosphite $(45 \mu \mathrm{L}, 250 \mu \mathrm{mol}, 5$ equiv.), dodecanenitrile (12 $\mu \mathrm{L}, 50 \mu \mathrm{mol}, 1.0$ equiv.) as internal standard and DIPEA (10.5 $\mu \mathrm{L}, 60 \mu \mathrm{mol}, 1.2$ equiv.) and $\mathrm{G} 1(10 \mathrm{mg} / \mathrm{mL})$. The gel mixture was irradiated for 2.5 hours, obtaining $90 \%$ product yield according to GC-FID analysis (75\% isolated yield as yellow oil). Using 5-chloro-2thiophenecarbonitrile, the gel mixture was irradiated for 4 hours, obtaining $87 \%$ product yield according to GC-FID analysis.

${ }^{1} \mathrm{H}$ NMR $\left(400 \mathrm{MHz}, \mathrm{CDCl}_{3}\right) \delta 7.64(\mathrm{t}, J=3.5 \mathrm{~Hz}, 1 \mathrm{H}), 7.59(\mathrm{dd}, J=8.1,3.8 \mathrm{~Hz}, 1 \mathrm{H}), 4.26$ $-4.07(\mathrm{~m}, 4 \mathrm{H}), 1.35(\mathrm{t}, J=7.1 \mathrm{~Hz}, 6 \mathrm{H}) \mathrm{ppm}$.

${ }^{13} \mathrm{C}$ NMR $\left(101 \mathrm{MHz}, \mathrm{CDCl}_{3}\right) \delta 137.6(\mathrm{CH}, \mathrm{d}, J=16.8 \mathrm{~Hz}), 136.8(\mathrm{C}, \mathrm{d}, J=204.5 \mathrm{~Hz})$, $135.7(\mathrm{CH}, \mathrm{d}, J=10.7 \mathrm{~Hz}), 116.8(\mathrm{C}, \mathrm{d}, J=10.0 \mathrm{~Hz}), 113.1(\mathrm{C}, \mathrm{d}, J=3.0 \mathrm{~Hz}), 63.6\left(\mathrm{CH}_{2}\right.$, $\mathrm{d}, J=5.6 \mathrm{~Hz}), 16.4\left(\mathrm{CH}_{3}, \mathrm{~d}, J=6.5 \mathrm{~Hz}\right) \mathrm{ppm}$.

31P NMR (162 MHz, $\left.\mathrm{CDCl}_{3}\right) \delta 7.48$ (s) ppm.

HRMS (EI): $\mathrm{m} / \mathrm{z}(\mathrm{M}+\mathrm{H})^{+}=$calcd. for $\mathrm{C}_{9} \mathrm{H}_{12} \mathrm{NO}_{3}$ PS: 246.0348 , found: 246.0347 .

\section{3b. Dimethyl (5-cyanothiophen-2-yl)phosphonate}<smiles>COP(=O)(OC)c1ccc(C#N)s1</smiles>

The compound was prepared according to the general procedure using 9-10-dicyanoanthracene (1.2 mg, $5 \mu \mathrm{mol}, 0.1$ equiv.), 5 bromo-2-thiophenecarbonitrile $(5.7 \mu \mathrm{L}, 50 \mu \mathrm{mol}, 1.0$ equiv.), trimethylphosphite $(30.4 \mu \mathrm{L}, 250 \mu \mathrm{mol}, 5$ equiv.), dodecanenitrile (12 $\mu \mathrm{L}, 50 \mu \mathrm{mol}, 1.0$ equiv.) as internal standard and DIPEA $(10.5 \mu \mathrm{L}, 60 \mu \mathrm{mol}, 1.2$ equiv.) and $\mathrm{G} 1(10 \mathrm{mg} / \mathrm{mL})$. The gel mixture was irradiated for 2 hours, obtaining $97 \%$ product yield according to GC-FID analysis ( $80 \%$ isolated yield as yellow oil).

${ }^{1} \mathrm{H}$ NMR $\left(400 \mathrm{MHz}, \mathrm{CDCl}_{3}\right) \delta 7.68-7.64(\mathrm{~m}, 1 \mathrm{H}), 7.61$ (dd, J = 8.1, 3.8 Hz, 1H), 3.84 (s, $3 \mathrm{H}), 3.81(\mathrm{~s}, 3 \mathrm{H}) \mathrm{ppm}$.

${ }^{13} \mathrm{C}$ NMR $\left(101 \mathrm{MHz}, \mathrm{CDCl}_{3}\right) \delta 137.7(\mathrm{CH}, \mathrm{d}, J=17.0 \mathrm{~Hz}), 136.2(\mathrm{CH}, \mathrm{d}, J=10.8 \mathrm{~Hz})$, $135.0(\mathrm{C}, \mathrm{d}, J=206.0 \mathrm{~Hz}), 53.7\left(\mathrm{CH}_{3}, \mathrm{dd}, J=5.5,2.2 \mathrm{~Hz}\right) \mathrm{ppm}$.

${ }^{31} \mathrm{P}$ NMR (162 MHz, $\left.\mathrm{CDCl}_{3}\right) \delta 10.47$ (s) ppm.

HRMS (El): $\mathrm{m} / \mathrm{z}(\mathrm{M}+\mathrm{H})^{+}=$calcd. for $\mathrm{C}_{7} \mathrm{H}_{8} \mathrm{NO}_{3} \mathrm{PS}: 218.0035$, found: 218.0036 .

\section{3c. Diphenyl (5-cyanothiophen-2-yl)phosphonate}<smiles>N#Cc1ccc(P(=O)(Oc2ccccc2)Oc2ccccc2)s1</smiles>

The compound was prepared according to the general procedure using 9-10-dicyanoanthracene (1.2 mg, $5 \mu \mathrm{mol}, 0.1$ equiv.), 5bromo-2-thiophenecarbonitrile $(5.7 \mu \mathrm{L}, 50 \mu \mathrm{mol}, 1.0$ equiv.), triphenylphosphite $(67.5 \mu \mathrm{L}, 250 \mu \mathrm{mol}, 5$ equiv.), dodecanenitrile $(12 \mu \mathrm{L}, 50 \mu \mathrm{mol}, 1.0$ equiv.) as internal standard and DIPEA (10.5 $\mu \mathrm{L}, 60 \mu \mathrm{mol}, 1.2$ equiv.) and $\mathrm{G} 1$ (10 $\mathrm{mg} / \mathrm{mL}$ ). The gel mixture was irradiated for 10 hours, obtaining $60 \%$ product yield according to GC-FID analysis ( $45 \%$ isolated yield as yellow powder).

${ }^{1} \mathrm{H}$ NMR $\left(400 \mathrm{MHz}, \mathrm{CDCl}_{3}\right) \delta 7.71(\mathrm{dd}, J=8.4,3.8 \mathrm{~Hz}, 1 \mathrm{H}), 7.64(\mathrm{t}, J=3.7 \mathrm{~Hz}, 1 \mathrm{H}), 7.35$ (dd, $J=11.1,4.7 \mathrm{~Hz}, 4 \mathrm{H}$ ), 7.21 (dd, $J=11.4,4.0 \mathrm{~Hz}, 6 \mathrm{H}$ ) ppm. 
${ }^{13} \mathrm{C}$ NMR (101 MHz, CDCl $\left.)_{3}\right) \delta 149.9(\mathrm{C}, \mathrm{d}, J=9.7 \mathrm{~Hz}), 137.6(\mathrm{CH}, \mathrm{d}, J=15.6 \mathrm{~Hz}), 137.3$ $(\mathrm{CH}, \mathrm{d}, J=11.3 \mathrm{~Hz}), 134.2(\mathrm{C}, \mathrm{d}, J=212.8 \mathrm{~Hz}), 130.2(\mathrm{CH}, \mathrm{s}), 126.1(\mathrm{CH}, \mathrm{d}, J=1.2 \mathrm{~Hz})$, $120.57(\mathrm{CH}, \mathrm{d}, J=4.7 \mathrm{~Hz}), 118.2(\mathrm{C}, \mathrm{d}, J=10.7 \mathrm{~Hz}), 112(\mathrm{C}, \mathrm{d}, J=3.0 \mathrm{~Hz}) \mathrm{ppm}$.

${ }^{31} \mathrm{P}$ NMR $\left(162 \mathrm{MHz}, \mathrm{CDCl}_{3}\right) \delta-0.18$ (s) ppm.

HRMS (El): $\mathrm{m} / \mathrm{z}(\mathrm{M}+\mathrm{H})^{+}=$calcd. for $\mathrm{C}_{17} \mathrm{H}_{12} \mathrm{NO}_{3} \mathrm{PS}: 342.0348$, found: 342.0344 .

\section{3d. Diphenyl (5-acetylthiophen-2-yl)phosphonate}<smiles>CCOP(=O)(OCC)c1ccc(C(C)=O)s1</smiles>

The compound (CAS: 1119779-20-2) was prepared according to the general procedure using 9-10-dicyanoanthracene $(1.2 \mathrm{mg}, 5$ $\mu \mathrm{mol}, 0.1$ equiv.), 2-acetyl-5-bromothiophene ( $4 \mathrm{mg}, 50 \mu \mathrm{mol}, 1.0$ equiv.), triethylphosphite (45 $\mu \mathrm{L}, 250 \mu \mathrm{mol}, 5$ equiv.), dodecanenitrile (12 $\mu \mathrm{L}, 50 \mu \mathrm{mol}, 1.0$ equiv.) as internal standard and DIPEA (10.5 $\mu \mathrm{L}, 60 \mu \mathrm{mol}, 1.2$ equiv.) and $\mathrm{G} 1(10 \mathrm{mg} / \mathrm{mL})$. The gel mixture was irradiated for 4 hours, obtaining $91 \%$ product yield according to GC-FID analysis $(78 \%$ isolated yield as yellow oil). Using 2-acetyl-5-chlorothiophene and 20 equivalents of triethylphosphite, the gel mixture was irradiated for 24 hours, obtaining $58 \%$ product yield according to GC-FID analysis.

${ }^{1} \mathrm{H}$ NMR $\left(400 \mathrm{MHz}, \mathrm{CDCl}_{3}\right) \delta 7.68(\mathrm{t}, J=3.3 \mathrm{~Hz}, 1 \mathrm{H}), 7.63(\mathrm{dd}, J=7.9,3.7 \mathrm{~Hz}, 1 \mathrm{H}), 4.27$ $-4.06(\mathrm{~m}, 4 \mathrm{H}), 2.59(\mathrm{~s}, 3 \mathrm{H}), 1.35(\mathrm{t}, J=7.1 \mathrm{~Hz}, 6 \mathrm{H}) \mathrm{ppm}$.

${ }^{13} \mathrm{C}$ NMR (101 MHz, $\left.\mathrm{CDCl}_{3}\right) \delta 190.6(\mathrm{C}, \mathrm{d}, J=1.6 \mathrm{~Hz}), 150.6(\mathrm{C}, \mathrm{d}, J=7.2 \mathrm{~Hz}), 136.7$ $(\mathrm{CH}, \mathrm{d}, J=11.5 \mathrm{~Hz}), 136.4(\mathrm{C}, \mathrm{d}, J=203.1 \mathrm{~Hz}), 132.1(\mathrm{CH}, \mathrm{d}, J=17.0 \mathrm{~Hz}), 63.2\left(\mathrm{CH}_{2}\right.$, $\mathrm{d}, J=5.5 \mathrm{~Hz}), 27.3\left(\mathrm{CH}_{3}, \mathrm{~s}\right), 16.4\left(\mathrm{CH}_{3}, \mathrm{~d}, J=6.6 \mathrm{~Hz}\right) \mathrm{ppm}$.

31P NMR (162 MHz, $\left.\mathrm{CDCl}_{3}\right) \delta 9.61$ (s) ppm.

HRMS (EI): $\mathrm{m} / \mathrm{z}(\mathrm{M}+\mathrm{H})^{+}=$calcd. for $\mathrm{C}_{10} \mathrm{H}_{15} \mathrm{O}_{4} \mathrm{PS}: 263.0501$, found: 263.0505 .

\section{3e. Diethyl (5-(trifluoromethyl)thiophen-2-yl)phosphonate}<smiles>CCOP(=O)(OCC)c1ccc(C(F)(F)F)s1</smiles>

The compound was prepared according to the general procedure using 9-10-dicyanoanthracene (1.2 mg, 5 mol, 0.1 equiv.), 2bromo-5-(trifluoromethyl)thiophene $(7.2 \mu \mathrm{L}, 50 \mu \mathrm{mol}, 1.0$ equiv.), triethylphosphite $(45 \mu \mathrm{L}, 250 \mu \mathrm{mol}, 5$ equiv.), dodecanenitrile (12 $\mu \mathrm{L}, 50 \mu \mathrm{mol}, 1.0$ equiv.) as internal standard and DIPEA (10.5 $\mu \mathrm{L}, 60 \mu \mathrm{mol}, 1.2$ equiv.) and $\mathrm{G} 1(10 \mathrm{mg} / \mathrm{mL})$. The gel mixture was irradiated for 4 hours, obtaining $94 \%$ product yield according to GC-FID analysis ( $71 \%$ isolated yield as yellow oil).

${ }^{1} \mathrm{H}$ NMR $\left(400 \mathrm{MHz}, \mathrm{CDCl}_{3}\right) \delta 7.58$ (ddd, $\left.J=8.1,3.7,1.2 \mathrm{~Hz}, 1 \mathrm{H}\right), 7.48$ (td, $J=3.6,1.0$ $\mathrm{Hz}, 1 \mathrm{H}), 4.26-4.07(\mathrm{~m}, 4 \mathrm{H}), 1.35(\mathrm{td}, J=7.1,0.4 \mathrm{~Hz}, 6 \mathrm{H}) \mathrm{ppm}$.

${ }^{13} \mathrm{C}$ NMR $\left(101 \mathrm{MHz}, \mathrm{CDCl}_{3}\right) \delta 138.6(\mathrm{C}, \mathrm{dq}, J=38.6,8.5 \mathrm{~Hz}), 135.9(\mathrm{CH}, \mathrm{d}, J=11.3 \mathrm{~Hz})$, $132.9(\mathrm{C}, \mathrm{dd}, J=206.1,1.0 \mathrm{~Hz}), 129.7-128.9(\mathrm{CH}, \mathrm{m}), 122.0\left(\mathrm{CF}_{3}, \mathrm{dq}, J=269.8,2.9\right.$ $\mathrm{Hz}), 63.3(\mathrm{~d}, J=5.5 \mathrm{~Hz}), 16.4(\mathrm{~d}, J=6.6 \mathrm{~Hz}) \mathrm{ppm}$.

${ }^{19} \mathrm{~F}$ NMR $\left(376 \mathrm{MHz}, \mathrm{CDCl}_{3}\right) \delta-55.65$ (s) ppm.

${ }^{31} \mathrm{P}$ NMR $\left(162 \mathrm{MHz}, \mathrm{CDCl}_{3}\right) \delta 8.98(\mathrm{~s}) \mathrm{ppm}$.

HRMS (EI): $\mathrm{m} / \mathrm{z}(\mathrm{M}+\mathrm{H})^{+}=$calcd. for $\mathrm{C}_{9} \mathrm{H}_{12} \mathrm{~F}_{3} \mathrm{O}_{3} \mathrm{PS}: 289.0270$, found: 289.0271 .

\section{3f. Diethyl (5-methylthiophen-2-yl)phosphonate}<smiles>CCOP(=O)(OCC)c1ccc(C)s1</smiles>

The compound was prepared according to the general procedure using 9-10-dicyanoanthracene (2.4 mg, $10 \mu \mathrm{mol}, 0.2$ equiv.), 2bromo-5-methylthiophene $(6.0 \mu \mathrm{L}, 50 \mu \mathrm{mol}, 1.0$ equiv.), triethylphosphite $(180 \mu \mathrm{L}, 1000 \mu \mathrm{mol}, 20$ equiv.), dodecanenitrile (12 $\mu \mathrm{L}, 50 \mu \mathrm{mol}, 1.0$ equiv.) as internal standard and DIPEA (10.5 $\mu \mathrm{L}, 60 \mu \mathrm{mol}, 1.2$ 
equiv.) and $\mathrm{G} 1$ (10 $\mathrm{mg} / \mathrm{mL})$. The gel mixture was irradiated for 24 hours, obtaining $42 \%$ product yield according to GC-FID analysis (25\% isolated yield as yellow oil).

${ }^{1} \mathrm{H}$ NMR $\left(400 \mathrm{MHz}, \mathrm{CDCl}_{3}\right) \delta 7.47(\mathrm{dd}, J=8.5,3.5 \mathrm{~Hz}, 1 \mathrm{H}), 6.83(\mathrm{td}, J=3.5,1.0 \mathrm{~Hz}, 1 \mathrm{H})$, $4.21-4.03(\mathrm{~m}, 4 \mathrm{H}), 2.54(\mathrm{~d}, J=0.8 \mathrm{~Hz}, 3 \mathrm{H}), 1.33(\mathrm{t}, J=7.1 \mathrm{~Hz}, 6 \mathrm{H}) \mathrm{ppm}$.

${ }^{13} \mathrm{C} \mathrm{NMR}\left(101 \mathrm{MHz}, \mathrm{CDCl}_{3}\right) \delta 149.1(\mathrm{C}, \mathrm{d}, J=7.1 \mathrm{~Hz}), 137.5(\mathrm{CH}, \mathrm{d}, J=11.6 \mathrm{~Hz}), 126.8$ $(\mathrm{CH}, \mathrm{d}, J=17.1 \mathrm{~Hz}), 124.1(\mathrm{C}, \mathrm{d}, J=213.1 \mathrm{~Hz}), 62.6\left(\mathrm{CH}_{2}, \mathrm{~d}, J=5.3 \mathrm{~Hz}\right), 16.4\left(\mathrm{CH}_{3}, \mathrm{~d}\right.$, $J=6.7 \mathrm{~Hz}), 15.5\left(\mathrm{CH}_{3}, \mathrm{~s}\right)$.

${ }^{31}$ P NMR (162 MHz, $\left.\mathrm{CDCl}_{3}\right) \delta 12.09$ (s) ppm.

HRMS (El): $\mathrm{m} / \mathrm{z}(\mathrm{M}+\mathrm{H})^{+}=$calcd. for $\mathrm{C}_{9} \mathrm{H}_{15} \mathrm{O}_{3} \mathrm{PS}: 235.0552$, found: 235.0554 .

\section{3g. Tetraethyl thiophene-2,5-diylbis(phosphonate)}<smiles>CCOP(=O)(OCC)c1ccc(P(=O)(OCC)OCC)s1</smiles>

The compound (CAS: 100651-98-7) was prepared according to the general procedure using 9-10-dicyanoanthracene (1.2 $\mathrm{mg}, 5$ $\mu \mathrm{mol}, 0.1$ equiv.), 2,5-dibromothiophene $(5.9 \mu \mathrm{L}, 50 \mu \mathrm{mol}, 1.0$ equiv.), triethylphosphite (180 $\mu \mathrm{L}, 1000 \mu \mathrm{mol}, 20$ equiv.), dodecanenitrile (12 $\mu \mathrm{L}, 50 \mu \mathrm{mol}, 1.0$ equiv.) as internal standard and DIPEA (10.5 $\mu \mathrm{L}$, $60 \mu \mathrm{mol}, 1.2$ equiv.) and $\mathrm{G} 1(10 \mathrm{mg} / \mathrm{mL})$. The gel mixture was irradiated for 10 hours, obtaining $96 \%$ product yield according to GC-FID analysis ( $81 \%$ isolated yield as colorless oil). Using 2,5-dichlorothiophene (5.4 $\mu \mathrm{L}, 50 \mu \mathrm{mol}, 1.0$ equiv.) and 9-10dicyanoanthracene $(2.4 \mathrm{mg}, 10 \mu \mathrm{mol}, 0.2$ equiv.), the gel mixture was irradiated for 10 hours, obtaining $73 \%$ product yield according to GC-FID analysis.

${ }^{1} \mathrm{H}$ NMR $\left(400 \mathrm{MHz}, \mathrm{CDCl}_{3}\right) \delta 7.65(\mathrm{~d}, J=4.8 \mathrm{~Hz}, 1 \mathrm{H}), 7.64(\mathrm{~d}, J=4.8 \mathrm{~Hz}, 1 \mathrm{H}), 4.27-$ $4.06(\mathrm{~m}, 8 \mathrm{H}), 1.35(\mathrm{t}, J=7.1 \mathrm{~Hz}, 12 \mathrm{H}) \mathrm{ppm}$.

${ }^{13} \mathrm{C}$ NMR (101 MHz, CDCl $)_{3} \delta 136.8-136.4(\mathrm{CH}, \mathrm{m}), 136.5(\mathrm{C}, \mathrm{dd}, J=203.2,6.2 \mathrm{~Hz})$, $63.3\left(\mathrm{CH}_{2}\right.$, dd, $\left.J=4.1,1.0 \mathrm{~Hz}\right), 16.4\left(\mathrm{CH}_{3}\right.$, dd, $\left.J=4.8,1.7 \mathrm{~Hz}\right) \mathrm{ppm}$.

31P NMR (162 MHz, $\left.\mathrm{CDCl}_{3}\right) \delta 9.55$ (s) ppm.

HRMS (EI): $\mathrm{m} / \mathrm{z}(\mathrm{M}+\mathrm{H})^{+}=$calcd. for $\mathrm{C}_{12} \mathrm{H}_{22} \mathrm{O}_{6} \mathrm{P}_{2} \mathrm{~S}: 357.0685$, found: 357.0683 .

\section{3h. Diethyl (5-cyanothiophen-3-yl)phosphonate}<smiles>CCOP(=O)(OCC)c1csc(C#N)c1</smiles>

The compound was prepared according to the general procedure using 9-10-dicyanoanthracene (2.4 mg, $10 \mu \mathrm{mol}, 0.2$ equiv.), 4-bromo-2thiophenecarbonitrile $(9.4 \mathrm{mg}, 50 \mu \mathrm{mol}, 1.0$ equiv.), triethylphosphite (180 $\mu \mathrm{L}, 1000 \mu \mathrm{mol}, 20$ equiv.), dodecanenitrile $(12 \mu \mathrm{L}, 50 \mu \mathrm{mol}, 1.0$ equiv.) as internal standard and DIPEA (10.5 $\mu \mathrm{L}, 60 \mu \mathrm{mol}, 1.2$ equiv.) and $\mathrm{G} 1(10 \mathrm{mg} / \mathrm{mL})$. The gel mixture was irradiated for 21 hours, obtaining $\mathbf{8 9} \%$ product yield according to GC-FID analysis ( $72 \%$ isolated yield as colorless oil).

${ }^{1} \mathrm{H}$ NMR $\left(400 \mathrm{MHz}, \mathrm{CDCl}_{3}\right) \delta 8.16(\mathrm{dd}, J=8.3,1.3 \mathrm{~Hz}, 1 \mathrm{H}), 7.81(\mathrm{dd}, J=4.5,1.3 \mathrm{~Hz}$, $1 \mathrm{H}), 4.25-4.04(\mathrm{~m}, 4 \mathrm{H}), 1.39-1.29(\mathrm{~m}, 6 \mathrm{H}) \mathrm{ppm}$.

${ }^{13} \mathrm{C}$ NMR $\left(101 \mathrm{MHz}, \mathrm{CDCl}_{3}\right) \delta 140.7(\mathrm{CH}, \mathrm{d}, J=16.7 \mathrm{~Hz}), 139.2(\mathrm{CH}, \mathrm{d}, J=16.3 \mathrm{~Hz})$, $132.0(\mathrm{C}, \mathrm{d}, J=199.5 \mathrm{~Hz}), 113.1(\mathrm{C}, \mathrm{s}), 112.3(\mathrm{C}, \mathrm{d}, J=21.9 \mathrm{~Hz}), 63.0\left(\mathrm{CH}_{2}, \mathrm{~d}, J=5.6\right.$ $\mathrm{Hz}), 16.5\left(\mathrm{CH}_{3}, \mathrm{~d}, J=6.4 \mathrm{~Hz}\right) \mathrm{ppm}$.

${ }^{31}$ P NMR (162 MHz, $\left.\mathrm{CDCl}_{3}\right) \delta 9.25$ (s) ppm.

HRMS (EI): $\mathrm{m} / \mathrm{z}(\mathrm{M}+\mathrm{H})^{+}=$calcd. for $\mathrm{C}_{9} \mathrm{H}_{12} \mathrm{NO}_{3} \mathrm{PS}: 246.0348$, found: 246.0344 .

\section{3i. Diethyl (5-cyanothiophen-3-yl)phosphonate}

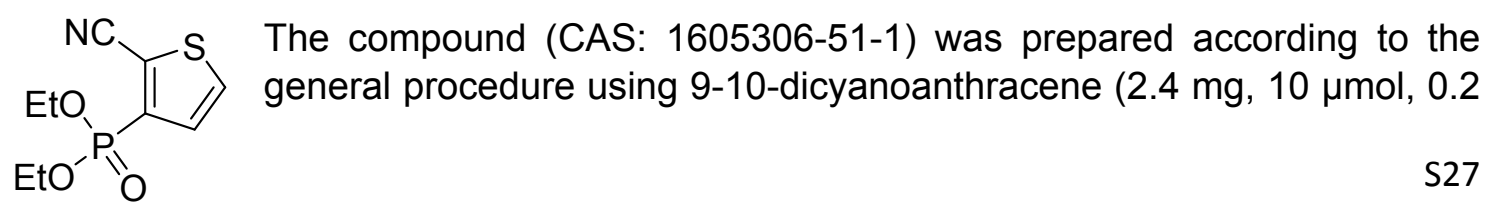


equiv.), 3-bromo-2-thiophenecarbonitrile ( $5.7 \mu \mathrm{L}, 50 \mu \mathrm{mol}, 1.0$ equiv.), triethylphosphite (180 $\mu \mathrm{L}, 1000 \mu \mathrm{mol}, 20$ equiv.), dodecanenitrile (12 $\mu \mathrm{L}, 50 \mu \mathrm{mol}, 1.0$ equiv.) as internal standard and DIPEA (10.5 $\mu \mathrm{L}, 60 \mu \mathrm{mol}, 1.2$ equiv.) and $\mathrm{G} 1(10 \mathrm{mg} / \mathrm{mL})$. The gel mixture was irradiated for 2.5 hours, obtaining $96 \%$ product yield according to GC-FID analysis ( $81 \%$ isolated yield as pale-yellow oil).

${ }^{1} \mathrm{H}$ NMR $\left(400 \mathrm{MHz}, \mathrm{CDCl}_{3}\right) \delta 7.64(\mathrm{dd}, J=5.1,3.0 \mathrm{~Hz}, 1 \mathrm{H}), 7.48(\mathrm{t}, J=5.0 \mathrm{~Hz}, 1 \mathrm{H}), 4.31$ -4.08 (m, 4H), 1.38 (td, $J=7.1,0.5 \mathrm{~Hz}, 6 \mathrm{H}) \mathrm{ppm}$.

${ }^{13} \mathrm{C}$ NMR $\left(101 \mathrm{MHz}, \mathrm{CDCl}_{3}\right) \delta 139.7(\mathrm{C}, \mathrm{d}, J=193.4 \mathrm{~Hz}), 132.4(\mathrm{CH}, \mathrm{d}, J=19.3 \mathrm{~Hz})$, $132.0(\mathrm{C}, \mathrm{d}, J=14.6 \mathrm{~Hz}), 116.1(\mathrm{C}, \mathrm{d}, J=11.3 \mathrm{~Hz}), 112.6(\mathrm{C}, \mathrm{d}, J=3.8 \mathrm{~Hz}), 63.4\left(\mathrm{CH}_{2}\right.$, $\mathrm{d}, J=5.9 \mathrm{~Hz}), 16.4\left(\mathrm{CH}_{3}, \mathrm{~d}, J=6.4 \mathrm{~Hz}\right) \mathrm{ppm}$.

${ }^{31} \mathrm{P}$ NMR (162 MHz, $\left.\mathrm{CDCl}_{3}\right) \delta 7.14$ (s) ppm.

HRMS (EI): $\mathrm{m} / \mathrm{z}(\mathrm{M}+\mathrm{H})^{+}=$calcd. for $\mathrm{C}_{9} \mathrm{H}_{12} \mathrm{NO}_{3} \mathrm{PS}: 246.0348$, found: 246.0344 .

\section{3j. Diethyl (4-cyanothiophen-2-yl)phosphonate}<smiles>CCOP(=O)(OCC)c1cc(C#N)cs1</smiles>

The compound was prepared according to the general procedure using 9-10-dicyanoanthracene (1.2 $\mathrm{mg}, 5 \mu \mathrm{mol}, 0.1$ equiv.), 5chloro-3-thiophenecarbonitrile (7.6 mg, $50 \mu \mathrm{mol}, 1.0$ equiv.), triethylphosphite $(45 \mu \mathrm{L}, 250 \mu \mathrm{mol}, 5$ equiv.), dodecanenitrile (12 $\mu \mathrm{L}, 50 \mu \mathrm{mol}, 1.0$ equiv.) as internal standard and DIPEA (10.5 $\mu \mathrm{L}, 60 \mu \mathrm{mol}, 1.2$ equiv.) and $\mathrm{G} 1(10 \mathrm{mg} / \mathrm{mL})$. The gel mixture was irradiated for 24 hours, obtaining $66 \%$ product yield according to GC-FID analysis ( $47 \%$ isolated yield as colorless oil).

${ }^{1} \mathrm{H}$ NMR $\left(400 \mathrm{MHz}, \mathrm{CDCl}_{3}\right) \delta 8.21$ (dd, $\left.J=5.6,1.2 \mathrm{~Hz}, 1 \mathrm{H}\right), 7.77$ (dd, $J=8.1,1.2 \mathrm{~Hz}$, $1 \mathrm{H}), 4.30-4.08(\mathrm{~m}, 4 \mathrm{H}), 1.36(\mathrm{td}, J=7.1,0.5 \mathrm{~Hz}, 6 \mathrm{H}) \mathrm{ppm}$.

${ }^{13} \mathrm{C}$ NMR $\left(101 \mathrm{MHz}, \mathrm{CDCl}_{3}\right) \delta 141.6(\mathrm{CH}, \mathrm{d}, J=6.4 \mathrm{~Hz}), 137.1(\mathrm{CH}, \mathrm{d}, J=12.4 \mathrm{~Hz})$, $132.2(\mathrm{C}, \mathrm{d}, J=208.5 \mathrm{~Hz}), 114.1$ (C, d, $J=1.3 \mathrm{~Hz}), 112.2$ (C, d, $J=21.2 \mathrm{~Hz}), 63.5\left(\mathrm{CH}_{2}\right.$, $\mathrm{d}, J=5.6 \mathrm{~Hz}), 16.4\left(\mathrm{CH}_{3}, \mathrm{~d}, J=6.4 \mathrm{~Hz}\right) \mathrm{ppm}$.

31P NMR (162 MHz, $\left.\mathrm{CDCl}_{3}\right) \delta 7.87$ (s) ppm.

HRMS (El): $\mathrm{m} / \mathrm{z}(\mathrm{M}+\mathrm{H})^{+}=$calcd. for $\mathrm{C}_{9} \mathrm{H}_{12} \mathrm{NO}_{3}$ PS: 246.0348 , found: 246.0344 .

\section{3k. Diethyl (4-cyanothiophen-3-yl)phosphonate}<smiles>CCOP(=O)(OCC)c1cscc1C#N</smiles>

The compound was prepared according to the general procedure using 9-10-dicyanoanthracene (1.2 mg, $5 \mu \mathrm{mol}, 0.1$ equiv.), 4-bromo-3thiophenecarbonitrile $(9.7 \mathrm{mg}, 50 \mu \mathrm{mol}, 1.0$ equiv.), triethylphosphite (180 $\mu \mathrm{L}, 250 \mu \mathrm{mol}, 5$ equiv.), dodecanenitrile (12 $\mu \mathrm{L}, 50 \mu \mathrm{mol}, 1.0$ equiv.) as internal standard and DIPEA (10.5 $\mu \mathrm{L}, 60 \mu \mathrm{mol}, 1.2$ equiv.) and $\mathrm{G} 1(10 \mathrm{mg} / \mathrm{mL})$. The gel mixture was irradiated for 8 hours, obtaining $63 \%$ product yield according to GC-FID analysis ( $51 \%$ isolated yield as colorless oil).

${ }^{1} \mathrm{H}$ NMR $\left(400 \mathrm{MHz}, \mathrm{CDCl}_{3}\right) \delta 8.13(\mathrm{dd}, J=8.5,3.1 \mathrm{~Hz}, 1 \mathrm{H}), 8.05(\mathrm{t}, J=3.1 \mathrm{~Hz}, 1 \mathrm{H}), 4.32$ $-4.06(\mathrm{~m}, 4 \mathrm{H}), 1.37$ (td, $J=7.1,0.4 \mathrm{~Hz}, 6 \mathrm{H}) \mathrm{ppm}$.

${ }^{13} \mathrm{C}$ NMR $\left(101 \mathrm{MHz}, \mathrm{CDCl}_{3}\right) \delta 138.3(\mathrm{CH}, \mathrm{d}, J=15.2 \mathrm{~Hz}), 138.0(\mathrm{CH}, \mathrm{d}, J=16.2 \mathrm{~Hz})$, 132.08 (C, d, $J=197.8 \mathrm{~Hz}$ ), 113.67 (C, s), 112.55 (C, d, $J=12.4 \mathrm{~Hz}), 63.27\left(\mathrm{CH}_{2}, \mathrm{~d}, J=\right.$ $5.9 \mathrm{~Hz}), 16.38\left(\mathrm{CH}_{3}, \mathrm{~d}, J=6.4 \mathrm{~Hz}\right)$.

${ }^{31} \mathrm{P}$ NMR (162 MHz, $\left.\mathrm{CDCl}_{3}\right) \delta 7.14$ (s) ppm.

HRMS (EI): $\mathrm{m} / \mathrm{z}(\mathrm{M}+\mathrm{H})^{+}=$calcd. for $\mathrm{C}_{9} \mathrm{H}_{12} \mathrm{NO}_{3} \mathrm{PS}: 246.0348$, found: 246.0346 .

\section{I. Diethyl (5-acetylthiophen-3-yl)phosphonate}

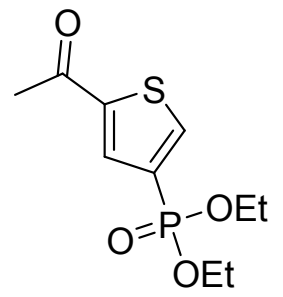

The compound was prepared according to the general procedure using 9-10-dicyanoanthracene (2.4 mg, $10 \mu \mathrm{mol}, 0.2$ equiv.), 2-acetyl-4- 
bromothiophene $(6.4 \mu \mathrm{L}, 50 \mu \mathrm{mol}, 1.0$ equiv.), triethylphosphite $(180 \mu \mathrm{L}, 1000 \mu \mathrm{mol}, 20$ equiv.), dodecanenitrile (12 $\mu \mathrm{L}, 50 \mu \mathrm{mol}, 1.0$ equiv.) as internal standard and DIPEA $(10.5 \mu \mathrm{L}, 60 \mu \mathrm{mol}, 1.2$ equiv.) and $\mathrm{G} 1(10 \mathrm{mg} / \mathrm{mL})$. The gel mixture was irradiated for 21 hours, obtaining $29 \%$ product yield according to GC-FID analysis ( $22 \%$ isolated yield as white powder). Using 2-acetyl-4-chlorothiophene, the gel mixture was irradiated for 24 hours, obtaining $12 \%$ product yield according to GC-FID analysis.

${ }^{1} \mathrm{H}$ NMR $\left(400 \mathrm{MHz}, \mathrm{CDCl}_{3}\right) \delta 8.32(\mathrm{dd}, J=5.7,1.3 \mathrm{~Hz}, 1 \mathrm{H}), 8.02(\mathrm{dd}, J=8.2,1.3 \mathrm{~Hz}$, $1 \mathrm{H}), 4.25-4.10(\mathrm{~m}, 4 \mathrm{H}), 2.55(\mathrm{~s}, 3 \mathrm{H}), 1.35(\mathrm{td}, J=7.1,0.5 \mathrm{~Hz}, 6 \mathrm{H}) \mathrm{ppm}$.

${ }^{13} \mathrm{C}$ NMR $\left(101 \mathrm{MHz}, \mathrm{CDCl}_{3}\right) \delta 190.6(\mathrm{C}, \mathrm{s}), 146.7$ (C, d, $\left.J=15.6 \mathrm{~Hz}\right), 141.8(\mathrm{CH}, \mathrm{d}, J=$ $16.2 \mathrm{~Hz}), 134.0(\mathrm{CH}, \mathrm{d}, J=15.8 \mathrm{~Hz}), 131.4(\mathrm{C}, \mathrm{d}, J=197.0 \mathrm{~Hz}), 62.7\left(\mathrm{CH}_{2}, \mathrm{~d}, J=5.6\right.$ $\mathrm{Hz}), 27.0\left(\mathrm{CH}_{3}, \mathrm{~s}\right), 16.5\left(\mathrm{CH}_{3}, \mathrm{~d}, J=6.5 \mathrm{~Hz}\right) \mathrm{ppm}$.

${ }^{31}$ P NMR (162 MHz, $\left.\mathrm{CDCl}_{3}\right) \delta 9.80$ (s) ppm.

HRMS (EI): $\mathrm{m} / \mathrm{z}(\mathrm{M}+\mathrm{H})^{+}=$calcd. for $\mathrm{C}_{10} \mathrm{H}_{15} \mathrm{O}_{4} \mathrm{PS}: 263.0501$, found: 263.0497 .

\section{3m. Diethyl (2-acetylthiophen-3-yl)phosphonate}<smiles>CCOP(=O)(OCC)c1ccsc1C(C)=O</smiles>

The compound was prepared according to the general procedure using 9-10-dicyanoanthracene (1.2 mg, $5 \mu \mathrm{mol}, 0.1$ equiv.), 2-acetyl-3bromothiophene $(10.6 \mathrm{mg}, 50 \mu \mathrm{mol}, 1.0$ equiv.), triethylphosphite $(45 \mu \mathrm{L}$, $250 \mu \mathrm{mol}, 5$ equiv.), dodecanenitrile $(12 \mu \mathrm{L}, 50 \mu \mathrm{mol}, 1.0$ equiv.) as internal standard and DIPEA (10.5 $\mu \mathrm{L}, 60 \mu \mathrm{mol}, 1.2$ equiv.) and G1 (10 $\mathrm{mg} / \mathrm{mL}$ ). The gel mixture was irradiated for 8 hours, obtaining $89 \%$ product yield according to GC-FID analysis (70\% isolated yield as yellow oil). Using 2acetyl-3-chlorothiophene, the gel mixture was irradiated for 24 hours, obtaining $\mathbf{4 8 \%}$ product yield according to GC-FID analysis.

${ }^{1} \mathrm{H}$ NMR $\left(400 \mathrm{MHz}, \mathrm{CDCl}_{3}\right) \delta 7.54(\mathrm{dd}, J=5.0,2.8 \mathrm{~Hz}, 1 \mathrm{H}), 7.51(\mathrm{t}, J=4.9 \mathrm{~Hz}, 1 \mathrm{H}), 4.32$ $-4.19(\mathrm{~m}, 4 \mathrm{H}), 2.71(\mathrm{~s}, 3 \mathrm{H}), 1.36(\mathrm{td}, J=7.1,0.5 \mathrm{~Hz}, 6 \mathrm{H}) \mathrm{ppm}$.

${ }^{13} \mathrm{C}$ NMR $\left(101 \mathrm{MHz}, \mathrm{CDCl}_{3}\right) \delta 190.4(\mathrm{C}, \mathrm{d}, J=2.6 \mathrm{~Hz}), 149.1(\mathrm{C}, \mathrm{d}, J=15.9 \mathrm{~Hz}), 134.9$ $(\mathrm{CH}, \mathrm{d}, J=14.2 \mathrm{~Hz}), 132.2(\mathrm{C}, \mathrm{d}, J=194.0 \mathrm{~Hz}), 130.31(\mathrm{CH}, \mathrm{d}, J=19.6 \mathrm{~Hz}), 63.02\left(\mathrm{CH}_{2}\right.$, $\mathrm{d}, J=6.0 \mathrm{~Hz}), 29.4\left(\mathrm{CH}_{3}, \mathrm{~d}, J=1.6 \mathrm{~Hz}\right), 16.5\left(\mathrm{CH}_{3}, \mathrm{~d}, J=6.5 \mathrm{~Hz}\right) \mathrm{ppm}$.

${ }^{31} \mathrm{P}$ NMR $\left(162 \mathrm{MHz}, \mathrm{CDCl}_{3}\right) \delta 7.14$ (s) ppm.

HRMS (EI): $\mathrm{m} / \mathrm{z}(\mathrm{M}+\mathrm{H})^{+}=$calcd. for $\mathrm{C}_{10} \mathrm{H}_{15} \mathrm{O}_{4} \mathrm{PS}: 263.0501$, found: 263.0498 .

\section{3n. Diethyl (4-acetylthiophen-2-yl)phosphonate}<smiles>CCOP(=O)(OCC)c1cc(C(C)=O)cs1</smiles>

The compound was prepared according to the general procedure using 9-10-dicyanoanthracene ( $1.2 \mathrm{mg}, 5 \mu \mathrm{mol}, 0.1$ equiv.), 3acetyl-5-chlorothiophene $(8.0 \mathrm{mg}, 50 \mu \mathrm{mol}, 1.0$ equiv. $)$, triethylphosphite $(180 \mu \mathrm{L}, 1000 \mu \mathrm{mol}, 20$ equiv.), dodecanenitrile (12 $\mu \mathrm{L}, 50 \mu \mathrm{mol}, 1.0$ equiv.) as internal standard and DIPEA (10.5 $\mu \mathrm{L}, 60 \mu \mathrm{mol}, 1.2$ equiv.) and $\mathrm{G} 1(10 \mathrm{mg} / \mathrm{mL})$. The gel mixture was irradiated for 24 hours, obtaining $\mathbf{2 6 \%}$ product yield according to GC-FID analysis (19\% isolated yield as white powder).

${ }^{1} \mathrm{H}$ NMR $\left(400 \mathrm{MHz}, \mathrm{CDCl}_{3}\right) \delta 8.17(\mathrm{dd}, \mathrm{J}=8.0,1.2 \mathrm{~Hz}, 1 \mathrm{H}), 7.87(\mathrm{dd}, \mathrm{J}=4.5,1.2 \mathrm{~Hz}$, $1 \mathrm{H}), 4.21-4.06(\mathrm{~m}, 4 \mathrm{H}), 2.58(\mathrm{~s}, 3 \mathrm{H}), 1.35(\mathrm{t}, \mathrm{J}=7.2 \mathrm{~Hz}, 6 \mathrm{H}) \mathrm{ppm}$.

${ }^{13} \mathrm{C}$ NMR $\left(101 \mathrm{MHz}, \mathrm{CDCl}_{3}\right) \delta 191.7(\mathrm{C}, \mathrm{s}), 143.4(\mathrm{C}, \mathrm{d}, J=17.4 \mathrm{~Hz}), 138.7(\mathrm{CH}, \mathrm{d}, J=$ $6.8 \mathrm{~Hz}), 136.0(\mathrm{CH}, \mathrm{d}, J=12.0 \mathrm{~Hz}), 129.1(\mathrm{C}, \mathrm{d}, J=211.2 \mathrm{~Hz}), 63.2\left(\mathrm{CH}_{2}, \mathrm{~d}, J=5.4 \mathrm{~Hz}\right)$, $27.9\left(\mathrm{CH}_{3}, \mathrm{~s}\right), 16.4\left(\mathrm{CH}_{3}, \mathrm{~d}, J=6.6 \mathrm{~Hz}\right) \mathrm{ppm}$.

${ }^{31} \mathrm{P}$ NMR $\left(162 \mathrm{MHz}, \mathrm{CDCl}_{3}\right) \delta 10.98$ (s) ppm.

HRMS (EI): $\mathrm{m} / \mathrm{z}(\mathrm{M}+\mathrm{H})^{+}=$calcd. for $\mathrm{C}_{10} \mathrm{H}_{15} \mathrm{O}_{4} \mathrm{PS}: 263.0501$, found: 263.0502 
30. yl)methyl)carbamoyl)thiophen-2-yl)phosphonate<smiles>CCOP(=O)(OCC)c1ccc(C(=O)NC[C@H]2CN(c3ccc(N4CCOCC4=O)cc3)C(=O)O2)s1</smiles>

The compound was prepared according to the general procedure using 9-10-dicyanoanthracene (2.4 $\mathrm{mg}, \quad 10 \mu \mathrm{mol}, \quad 0.2$ equiv.), rivaroxaban $(21.8 \mathrm{mg}, 50 \mu \mathrm{mol}, 1.0$ equiv.), triethylphosphite $(180 \mu \mathrm{L}$, $1000 \mu \mathrm{mol}, 20$ equiv.), DIPEA (10.5 $\mu \mathrm{L}, 60 \mu \mathrm{mol}, 1.2$ equiv.) and $\mathrm{G} 1(10 \mathrm{mg} / \mathrm{mL})$. The gel mixture was irradiated for 24 hours, obtaining $70 \%(18.9 \mathrm{mg})$ isolated product yield as pale-yellow oil.

${ }^{1} \mathrm{H}$ NMR $\left(400 \mathrm{MHz}, \mathrm{CDCl}_{3}\right) \delta 7.72(\mathrm{t}, J=6.0 \mathrm{~Hz}, 1 \mathrm{H}), 7.58(\mathrm{t}, J=3.5 \mathrm{~Hz}, 1 \mathrm{H}), 7.55-7.50$ $(\mathrm{m}, 2 \mathrm{H}), 7.48(\mathrm{dd}, J=8.1,3.8 \mathrm{~Hz}, 1 \mathrm{H}), 7.33-7.28(\mathrm{~m}, 2 \mathrm{H}), 4.83(\mathrm{td}, J=11.5,5.1 \mathrm{~Hz}$, $1 \mathrm{H}), 4.32(\mathrm{~s}, 2 \mathrm{H}), 4.23-4.06(\mathrm{~m}, 4 \mathrm{H}), 4.06-3.98(\mathrm{~m}, 3 \mathrm{H}), 3.83(\mathrm{dd}, J=9.2,6.6 \mathrm{~Hz}$, $1 \mathrm{H}), 3.79-3.66(\mathrm{~m}, 4 \mathrm{H}), 1.38-1.25(\mathrm{~m}, 6 \mathrm{H}) \mathrm{ppm}$.

${ }^{13} \mathrm{C}$ NMR (101 MHz, CDCl $)$ $) 167.2(\mathrm{C}, \mathrm{s}), 162.1$ (C, d, J = 2.2 Hz), 154.6 (C, s), 145.8 (C, d, J = 8.0 Hz), $137.4(\mathrm{C}, \mathrm{s}), 136.9(\mathrm{C}, \mathrm{s}), 136.6(\mathrm{CH}, \mathrm{d}, J=11.4 \mathrm{~Hz}), 132.9$ (C, d, J = $205.3 \mathrm{~Hz}), 129.0(\mathrm{CH}, \mathrm{d}, J=17.2 \mathrm{~Hz}), 126.5(\mathrm{CH}, \mathrm{s}), 119.2(\mathrm{CH}, \mathrm{s}), 71.7(\mathrm{CH}, \mathrm{s}), 68.6$ $\left(\mathrm{CH}_{2}, \mathrm{~s}\right), 64.2\left(\mathrm{CH}_{2}, \mathrm{~s}\right), 63.3\left(\mathrm{CH}_{2}, \mathrm{dd}, \mathrm{J}=5.4,2.4 \mathrm{~Hz}\right), 49.9\left(\mathrm{CH}_{2}, \mathrm{~s}\right), 47.8\left(\mathrm{CH}_{2}, \mathrm{~s}\right), 42.6$ $\left(\mathrm{CH}_{2}, \mathrm{~s}\right), 16.4\left(\mathrm{CH}_{3}, \mathrm{~d}, J=6.7 \mathrm{~Hz}\right) \mathrm{ppm}$.

${ }^{31} \mathrm{P}$ NMR (162 MHz, $\left.\mathrm{CDCl}_{3}\right) \delta 9.80$ (s) ppm.

HRMS (El): $\mathrm{m} / \mathrm{z}(\mathrm{M}+\mathrm{H})^{+}=$calcd. for $\mathrm{C}_{23} \mathrm{H}_{28} \mathrm{~N}_{3} \mathrm{O}_{8}$ PS: 538.1374 , found: 538.1375 .

\section{FURANS}

\section{0a. Diethyl (5-cyanofuran-2-yl)phosphonate}<smiles>CCOP(=O)(OCC)c1ccc(C#N)o1</smiles>

The compound was prepared according to the general procedure using 9-10-dicyanoanthracene (1.2 mg, $5 \mu \mathrm{mol}, 0.1$ equiv.), 5bromo-2-furancarbonitrile (5 $\mu \mathrm{L}, \quad 50 \mu \mathrm{mol}, \quad 1.0$ equiv.), triethylphosphite $(45 \mu \mathrm{L}, 250 \mu \mathrm{mol}, 5$ equiv.), dodecanenitrile (12 $\mu \mathrm{L}, 50 \mu \mathrm{mol}, 1.0$ equiv.) as internal standard, DIPEA (10.5 $\mu \mathrm{L}, 60 \mu \mathrm{mol}, 1.2$ equiv.) and G1 $(10 \mathrm{mg} / \mathrm{mL})$. The gel mixture was irradiated for 3 hours, obtaining $85 \%$ product yield according to GC-FID analysis ( $77 \%$ isolated yield as yellow oil).

${ }^{1} \mathrm{H}$ NMR $\left(400 \mathrm{MHz}, \mathrm{CDCl}_{3}\right) \delta 7.17$ (dd, $\left.J=3.6,2.0 \mathrm{~Hz}, 1 \mathrm{H}\right), 7.14$ (dd, $J=3.6,2.2 \mathrm{~Hz}$, $1 \mathrm{H}), 4.32-4.10(\mathrm{~m}, 4 \mathrm{H}), 1.37(\mathrm{td}, J=7.1,0.5 \mathrm{~Hz}, 6 \mathrm{H}) \mathrm{ppm}$.

${ }^{13} \mathrm{C}$ NMR $\left(101 \mathrm{MHz}, \mathrm{CDCl}_{3}\right) \delta 150.4(\mathrm{C}, \mathrm{d}, J=232.0 \mathrm{~Hz}), 130.4(\mathrm{C}, \mathrm{d}, J=13.4 \mathrm{~Hz}), 122.4$ $(\mathrm{d}, J=23.2 \mathrm{~Hz}), 121.8(\mathrm{~d}, J=10.4 \mathrm{~Hz}), 110.5(\mathrm{C}, \mathrm{d}, J=2.5 \mathrm{~Hz}), 63.9(\mathrm{~d}, J=5.7 \mathrm{~Hz})$, $16.4(\mathrm{~d}, J=6.3 \mathrm{~Hz}) \mathrm{ppm}$.

${ }^{31} \mathrm{P}$ NMR (162 MHz, $\left.\mathrm{CDCl}_{3}\right) \delta 0.19$ (s) ppm.

HRMS (El): $\mathrm{m} / \mathrm{z}(\mathrm{M}+\mathrm{H})^{+}=$calcd. for $\mathrm{C}_{9} \mathrm{H}_{12} \mathrm{NO}_{4} \mathrm{P}: 230.0577$, found: 230.0574 .

\section{0b. Diethyl (5-acetylfuran-2-yl)phosphonate}

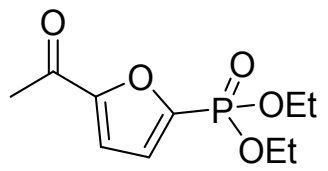

The compound (CAS: 261365-06-4) was prepared according to the general procedure using 9-10-dicyanoanthracene $(1.2 \mathrm{mg}, 5 \mu \mathrm{mol}$, 0.1 equiv.), 2-acetyl-5-bromofuran ( $9.9 \mathrm{mg}, 50 \mu \mathrm{mol}, 1.0$ equiv.), triethylphosphite $(45 \mu \mathrm{L}, 250 \mu \mathrm{mol}, 5$ equiv.), dodecanenitrile (12 $\mu \mathrm{L}, 50 \mu \mathrm{mol}, 1.0$ equiv.) as internal standard, DIPEA (10.5 $\mu \mathrm{L}, 60 \mu \mathrm{mol}, 1.2$ equiv.) and G1 $(10 \mathrm{mg} / \mathrm{mL})$. The gel mixture was irradiated for 4 hours, obtaining $96 \%$ product yield according to GC-FID analysis ( $82 \%$ isolated yield as pale-yellow oil). 
${ }^{1} \mathrm{H}$ NMR $\left(400 \mathrm{MHz}, \mathrm{CDCl}_{3}\right) \delta 7.16(\mathrm{dd}, J=3.6,2.4 \mathrm{~Hz}, 1 \mathrm{H}), 7.14(\mathrm{dd}, J=3.6,1.8 \mathrm{~Hz}$, $1 \mathrm{H}), 4.26-4.10(\mathrm{~m}, 4 \mathrm{H}), 2.51(\mathrm{~s}, 3 \mathrm{H}), 1.34(\mathrm{q}, J=6.9 \mathrm{~Hz}, 6 \mathrm{H}) \mathrm{ppm}$.

${ }^{13} \mathrm{C}$ NMR $\left(101 \mathrm{MHz}, \mathrm{CDCl}_{3}\right) \delta 187.3(\mathrm{C}, \mathrm{d}, J=1.0 \mathrm{~Hz}), 156.4(\mathrm{C}, \mathrm{d}, J=9.5 \mathrm{~Hz}), 147.9$ $(\mathrm{C}, \mathrm{d}, J=236.5 \mathrm{~Hz}), 123.3(\mathrm{CH}, \mathrm{d}, J=24.0 \mathrm{~Hz}), 116.0(\mathrm{CH}, \mathrm{d}, J=10.7 \mathrm{~Hz}), 63.5\left(\mathrm{CH}_{2}\right.$, $\mathrm{d}, J=5.6 \mathrm{~Hz}), 26.5\left(\mathrm{CH}_{3}, \mathrm{~d}, J=1.6 \mathrm{~Hz}\right), 16.4\left(\mathrm{CH}_{3}, \mathrm{~d}, J=6.2 \mathrm{~Hz}\right) \mathrm{ppm}$.

31P NMR (162 MHz, $\mathrm{CDCl}_{3}$ ) $\delta 2.15$ (s) ppm.

HRMS (El): $\mathrm{m} / \mathrm{z}(\mathrm{M}+\mathrm{H})^{+}=$calcd. for $\mathrm{C}_{10} \mathrm{H}_{15} \mathrm{O}_{5} \mathrm{P}: 247.0730$, found: 247.0729 .

Spectral data were consistent with the literature. ${ }^{1}$

\section{0c. Diethyl (5-methylfuran-2-yl)phosphonate}

$\mathrm{H}_{3} \mathrm{C} \quad \mathrm{O}$ The compound (CAS: 70519-53-8) was prepared according to the general procedure using 9-10-dicyanoanthracene $(2.4 \mathrm{mg}, 10$ OEt $\mu \mathrm{mol}, 0.2$ equiv.), 2-bromo-5-methylfuran $(5.5 \mu \mathrm{L}, 50 \mu \mathrm{mol}, 1.0$ equiv.), triethylphosphite (180 $\mu \mathrm{L}, 1000 \mu \mathrm{mol}, 20$ equiv.), dodecanenitrile (12 $\mu \mathrm{L}, 50$ $\mu \mathrm{mol}, 1.0$ equiv.) as internal standard, DIPEA (10.5 $\mu \mathrm{L}, 60 \mu \mathrm{mol}, 1.2$ equiv.) and $\mathrm{G} 1$ (10 $\mathrm{mg} / \mathrm{mL}$ ). The gel mixture was irradiated for 24 hours, obtaining $30 \%$ product yield according to GC-FID analysis ( $19 \%$ isolated yield as white powder).

${ }^{1} \mathrm{H}$ NMR $\left(400 \mathrm{MHz}, \mathrm{CDCl}_{3}\right) \delta 7.05$ (dd, $\left.J=2.9,2.2 \mathrm{~Hz}, 1 \mathrm{H}\right), 6.08$ (ddd, $J=3.4,2.7,0.9$ $\mathrm{Hz}, 1 \mathrm{H}), 4.26-4.16(\mathrm{~m}, 4 \mathrm{H}), 2.36(\mathrm{~s}, 3 \mathrm{H}), 1.34$ (td, $J=7.1,0.5 \mathrm{~Hz}, 6 \mathrm{H}) \mathrm{ppm}$.

${ }^{13} \mathrm{C}$ NMR $\left(101 \mathrm{MHz}, \mathrm{CDCl}_{3}\right) \delta 161.8(\mathrm{C}, \mathrm{d}, J=242.8 \mathrm{~Hz}), 143.2(\mathrm{C}, \mathrm{s}), 124.4(\mathrm{~d}, J=26.2$ $\mathrm{Hz}), 107.2(\mathrm{CH}, \mathrm{d}, J=11.8 \mathrm{~Hz}), 62.8\left(\mathrm{CH}_{2}, \mathrm{~d}, J=3.0 \mathrm{~Hz}\right), 16.4\left(\mathrm{CH}_{3}, \mathrm{~d}, J=7.04 \mathrm{~Hz}\right)$, $14.2\left(\mathrm{CH}_{3}, \mathrm{~s}\right) \mathrm{ppm}$.

${ }^{31}$ P NMR (162 MHz, $\left.\mathrm{CDCl}_{3}\right) \delta 5.02$ (s) ppm.

HRMS (EI): $\mathrm{m} / \mathrm{z}(\mathrm{M}+\mathrm{H})^{+}=$calcd. for $\mathrm{C}_{9} \mathrm{H}_{15} \mathrm{O}_{4} \mathrm{P}: 219.0781$, found 219.0779 .

\section{PYRROLE}

11a. Diethyl (5-acetyl-1-methyl-1H-pyrrol-2-yl)phosphonate<smiles></smiles>

The compound (CAS: 1394991-86-6) was prepared according to the general procedure using 9-10-dicyanoanthracene $(1.2 \mathrm{mg}, 5$ $\mu$ mol, 0.1 equiv.), 2-acetyl-5-bromo- $N$-methylpyrrole $(10.1 \mathrm{mg}, 50$ $\mu \mathrm{mol}, 1.0$ equiv.), triethylphosphite $(45 \mu \mathrm{L}, 250 \mu \mathrm{mol}, 5$ equiv.), dodecanenitrile (12 $\mu \mathrm{L}, 50 \mu \mathrm{mol}, 1.0$ equiv.) as internal standard, DIPEA (10.5 $\mu \mathrm{L}, 60$ $\mu \mathrm{mol}, 1.2$ equiv.) and $\mathrm{G} 1(10 \mathrm{mg} / \mathrm{mL})$. The gel mixture was irradiated for 4 hours, obtaining $92 \%$ product yield according to GC-FID analysis ( $76 \%$ isolated yield as paleyellow oil).

${ }^{1} \mathrm{H}$ NMR $\left(400 \mathrm{MHz}, \mathrm{CDCl}_{3}\right) \delta 6.91(\mathrm{t}, J=4.1 \mathrm{~Hz}, 1 \mathrm{H}), 6.76(\mathrm{dd}, J=4.1,3.2 \mathrm{~Hz}, 1 \mathrm{H}), 4.21$ $-4.10(\mathrm{~m}, 4 \mathrm{H}), 4.09(\mathrm{~d}, J=0.8 \mathrm{~Hz}, 3 \mathrm{H}), 2.48(\mathrm{~s}, 3 \mathrm{H}), 1.34(\mathrm{t}, J=7.1 \mathrm{~Hz}, 6 \mathrm{H}) \mathrm{ppm}$.

${ }^{13} \mathrm{C}$ NMR (101 MHz, CDCl $\left.)_{3}\right) \delta 190.0(\mathrm{C}, \mathrm{d}, J=1.3 \mathrm{~Hz}), 135.7$ (C, d, J = $\left.11.5 \mathrm{~Hz}\right), 128.2$ $(\mathrm{C}, \mathrm{d}, J=219.2 \mathrm{~Hz}), 119.6(\mathrm{CH}, \mathrm{d}, J=17.0 \mathrm{~Hz}), 118.1(\mathrm{CH}, \mathrm{d}, J=13.9 \mathrm{~Hz}), 62.8\left(\mathrm{CH}_{2}\right.$, $\mathrm{dd}, J=5.3,2.1 \mathrm{~Hz}), 36.1-36.0\left(\mathrm{CH}_{3}, \mathrm{~m}\right), 28.6-28.1\left(\mathrm{CH}_{3}, \mathrm{~m}\right), 16.4\left(\mathrm{CH}_{3}, \mathrm{~d}, J=6.5\right.$ $\mathrm{Hz}) \mathrm{ppm}$.

31P NMR (162 MHz, $\left.\mathrm{CDCl}_{3}\right) \delta 8.33$ (s) ppm.

HRMS (EI): $\mathrm{m} / \mathrm{z}(\mathrm{M}+\mathrm{H})^{+}=$calcd. for $\mathrm{C}_{11} \mathrm{H}_{18} \mathrm{NO}_{4} \mathrm{P}: 260.1046$, found: 260.1046 .

\footnotetext{
${ }^{1}$ Qun Dang, Srinivas Rao Kasibhatla, K. Raja Reddy, Tao Jiang, M. Rami Reddy, Scott C. Potter, James M. Fujitaki, Paul D. van Poelje, Jingwei Huang, William N. Lipscomb, and Mark D. Erion. J. Am. Chem. Soc. 2007, 129, 50, 15491-15502
} 
Spectral data were consistent with the literature. ${ }^{2}$

\section{SELENOPHENE}

\section{2a. Diethyl (5-acetylselenophen-2-yl)phosphonate}<smiles>CCOP(=O)(OCC)c1ccc(C(C)=O)s1</smiles>

The compound was prepared according to the general procedure using 9-10-dicyanoanthracene (1.2 mg, $5 \mu \mathrm{mol}, 0.1$ equiv.), 2-acetyl-

5-bromoselenophene (12.6 $\mathrm{mg}, 50 \mu \mathrm{mol}, 1.0$ equiv.), triethylphosphite $(45 \mu \mathrm{L}, 250 \mu \mathrm{mol}, 5$ equiv. $)$, dodecanenitrile $(12 \mu \mathrm{L}$, $50 \mu \mathrm{mol}, 1.0$ equiv.) as internal standard, DIPEA (10.5 $\mu \mathrm{L}, 60 \mu \mathrm{mol}, 1.2$ equiv.) and $\mathrm{G} 1$ $(10 \mathrm{mg} / \mathrm{mL}$ ). The gel mixture was irradiated for 4 hours, obtaining $86 \%$ product yield according to GC-FID analysis ( $71 \%$ isolated yield as yellow oil).

${ }^{1} \mathrm{H}$ NMR $\left(400 \mathrm{MHz}, \mathrm{CDCl}_{3}\right) \delta 7.92(\mathrm{dd}, J=8.4,3.0 \mathrm{~Hz}, 1 \mathrm{H}), 7.89(\mathrm{t}, J=2.9 \mathrm{~Hz}, 1 \mathrm{H}), 4.24$ $-4.08(\mathrm{~m}, 4 \mathrm{H}), 2.59(\mathrm{~s}, 3 \mathrm{H}), 1.34(\mathrm{td}, J=7.1,0.5 \mathrm{~Hz}, 6 \mathrm{H}) \mathrm{ppm}$.

${ }^{13} \mathrm{C}$ NMR (101 MHz, $\mathrm{CDCl}_{3}$ ) $\delta 192.0(\mathrm{C}, \mathrm{d}, J=1.2 \mathrm{~Hz}), 158.4(\mathrm{C}, \mathrm{d}, J=5.5 \mathrm{~Hz}), 142.4$ (C, d, $J=197.7 \mathrm{~Hz}), 139.2(\mathrm{CH}, \mathrm{d}, J=10.5 \mathrm{~Hz}), 134.5(\mathrm{CH}, \mathrm{d}, J=18.7 \mathrm{~Hz}), 63.2\left(\mathrm{CH}_{2}\right.$, dd, $J=5.1,2.2 \mathrm{~Hz}), 26.5\left(\mathrm{CH}_{3}, \mathrm{~d}, J=2.1 \mathrm{~Hz}\right), 16.4\left(\mathrm{CH}_{3}, \mathrm{~d}, J=6.6 \mathrm{~Hz}\right) \mathrm{ppm}$.

31P NMR (162 MHz, $\left.\mathrm{CDCl}_{3}\right) \delta 12.02$ (s) ppm.

HRMS (EI): $\mathrm{m} / \mathrm{z}(\mathrm{M}+\mathrm{H})^{+}=$calcd. for $\mathrm{C}_{10} \mathrm{H}_{15} \mathrm{O}_{4} \mathrm{PSe}: 310.9946$, found: 310.9944 .

\section{OXAZOLE}

\section{3a. Ethyl 2-(diethoxyphosphoryl)oxazole-5-carboxylate}<smiles>CCOC(=O)c1cnc(P(=O)(OCC)OCC)o1</smiles>

The compound was prepared according to the general procedure using 9-10-dicyanoanthracene $(1.2 \mathrm{mg}, 5 \mu \mathrm{mol}, 0.1$ equiv.), ethyl 2-chloro-1,3-oxazole-5-carboxylate $(6.6 \mu \mathrm{L}, 50$ $\mu \mathrm{mol}, 1.0$ equiv.), triethylphosphite $(45 \mu \mathrm{L}, 250 \mu \mathrm{mol}, 5$ equiv. $)$, dodecanenitrile (12 $\mu \mathrm{L}, 50 \mu \mathrm{mol}, 1.0$ equiv.) as internal standard, DIPEA (10.5 $\mu \mathrm{L}, 60$ $\mu \mathrm{mol}, 1.2$ equiv.) and $\mathrm{G} 1(10 \mathrm{mg} / \mathrm{mL})$. The gel mixture was irradiated for 15 hours, obtaining $91 \%$ product yield according to GC-FID analysis ( $76 \%$ isolated yield as paleyellow oil).

${ }^{1} \mathrm{H}$ NMR $\left(400 \mathrm{MHz}, \mathrm{CDCl}_{3}\right) \delta 7.84(\mathrm{~d}, J=1.2 \mathrm{~Hz}, 1 \mathrm{H}), 4.41(\mathrm{q}, J=7.1 \mathrm{~Hz}, 2 \mathrm{H}), 4.38-$ $4.30(\mathrm{~m}, 4 \mathrm{H}), 1.42(\mathrm{td}, J=7.1,0.7 \mathrm{~Hz}, 6 \mathrm{H}), 1.40(\mathrm{t}, J=7.1 \mathrm{~Hz}, 3 \mathrm{H}) \mathrm{ppm}$.

${ }^{13} \mathrm{C}$ NMR (101 MHz, $\left.\mathrm{CDCl}_{3}\right) \delta 158.4(\mathrm{C}, \mathrm{d}, J=266.2 \mathrm{~Hz}), 157.2(\mathrm{C}, \mathrm{d}, J=0.5 \mathrm{~Hz}), 145.0$ $(\mathrm{C}, \mathrm{d}, J=5.6 \mathrm{~Hz}), 133.9(\mathrm{CH}, \mathrm{d}, J=14.6 \mathrm{~Hz}), 64.9\left(\mathrm{CH}_{2}, \mathrm{~d}, J=5.9 \mathrm{~Hz}\right), 62.2\left(\mathrm{CH}_{2}, \mathrm{~s}\right)$, $16.4\left(\mathrm{CH}_{3}, \mathrm{~d}, J=6.3 \mathrm{~Hz}\right), 14.3\left(\mathrm{CH}_{3}, \mathrm{~s}\right) \mathrm{ppm}$.

31P NMR (162 MHz, $\left.\mathrm{CDCl}_{3}\right) \delta-4.19$ (s) ppm.

HRMS (EI): $\mathrm{m} / \mathrm{z}(\mathrm{M}+\mathrm{H})^{+}=$calcd. for $\mathrm{C}_{10} \mathrm{H}_{16} \mathrm{NO}_{6} \mathrm{P}: 278.0788$, found: 278.0787 .

\section{THIAZOLE}

\section{4a. Diethyl (5-cyanothiazol-2-yl)phosphonate}<smiles>CCOP(=O)(OCC)c1ncc(C#N)s1</smiles>

The compound was prepared according to the general procedure using 9-10-dicyanoanthracene ( $1.2 \mathrm{mg}, 5 \mu \mathrm{mol}, 0.1$ equiv.), ethyl 2bromo-1,3-thiazole-5-carbonitrile (9.5 mg, $50 \mu \mathrm{mol}, 1.0$ equiv.), triethylphosphite $(45 \mu \mathrm{L}, 250 \mu \mathrm{mol}, 5$ equiv.), dodecanenitrile (12 $\mu \mathrm{L}, 50 \mu \mathrm{mol}, 1.0$ equiv.) as internal standard, DIPEA (10.5 $\mu \mathrm{L}, 60 \mu \mathrm{mol}, 1.2$ equiv.) and

\footnotetext{
${ }^{2}$ Chang-Bing Xiang, Yong-Jun Bian, Xue-Rong Mao, and Zhi-Zhen Huang. J. Org. Chem. 2012, 77, 17, 7706-7710
} 
G1 $(10 \mathrm{mg} / \mathrm{mL})$. The reaction mixture was irradiated for 15 hours, obtaining $\mathbf{8 0} \%$ product yield according to GC-FID analysis ( $72 \%$ isolated yield as pale-yellow oil).

${ }^{1} \mathrm{H}$ NMR $\left(400 \mathrm{MHz}, \mathrm{CDCl}_{3}\right) \delta 8.49(\mathrm{~d}, \mathrm{~J}=1.5 \mathrm{~Hz}, 1 \mathrm{H}), 4.39-4.22(\mathrm{~m}, 4 \mathrm{H}), 1.39(\mathrm{td}, \mathrm{J}=$ $7.1,0.7 \mathrm{~Hz}, 6 \mathrm{H}) \mathrm{ppm}$.

${ }^{13} \mathrm{C}$ NMR $\left(101 \mathrm{MHz}, \mathrm{CDCl}_{3}\right) \delta 165.6(\mathrm{C}, \mathrm{d}, J=237.3 \mathrm{~Hz}), 153.8(\mathrm{CH}, \mathrm{d}, J=26.0 \mathrm{~Hz})$, $111.5(\mathrm{C}, \mathrm{d}, J=1.7 \mathrm{~Hz}), 111.0(\mathrm{C}, \mathrm{d}, J=1.5 \mathrm{~Hz}), 64.9\left(\mathrm{CH}_{2}, \mathrm{~d}, J=6.1 \mathrm{~Hz}\right), 16.4\left(\mathrm{CH}_{3}, \mathrm{~d}\right.$, $J=6.1 \mathrm{~Hz})$ ppm.

${ }^{31} \mathrm{P}$ NMR (162 MHz, $\left.\mathrm{CDCl}_{3}\right) \delta 0.51$ (s) ppm.

HRMS (El): $\mathrm{m} / \mathrm{z}(\mathrm{M}+\mathrm{H})^{+}=$calcd. for $\mathrm{C}_{8} \mathrm{H}_{11} \mathrm{~N}_{2} \mathrm{O}_{3}$ PS: 247.0301 , found: 247.0300 .

\section{6-MEMBERS HETEROARENES}

15a. Diethyl (4-cyanophenyl)phosphonate<smiles>CCOP(=O)(OCC)c1ccc(C#N)cc1</smiles>

The compound (CAS: 28255-72-3) was prepared according to the general procedure using 9-10-dicyanoanthracene $(1.2 \mathrm{mg}, 5 \mu \mathrm{mol}$, 0.1 equiv.), 4-bromobenzonitrile ( $9.2 \mathrm{mg}, 50 \mu \mathrm{mol}, 1.0$ equiv.), triethylphosphite $(45 \mu \mathrm{L}, 250 \mu \mathrm{mol}, 5$ equiv.), dodecanenitrile $(12 \mu \mathrm{L}$, $50 \mu \mathrm{mol}, 1.0$ equiv. $)$ as internal standard, DIPEA $(10.5 \mu \mathrm{L}, 60 \mu \mathrm{mol}$, 1.2 equiv.) and $\mathrm{G} 1$ ( $10 \mathrm{mg} / \mathrm{mL})$. The reaction mixture was irradiated for 2 hours, obtaining $\mathbf{9 8 \%}$ product yield according to GC-FID analysis (90\% isolated yield as colorless oil).

${ }^{1} \mathrm{H}$ NMR $\left(400 \mathrm{MHz}, \mathrm{CDCl}_{3}\right) \delta 7.92(\mathrm{dd}, \mathrm{J}=13.1,8.5 \mathrm{~Hz}, 1 \mathrm{H}), 7.75$ (dd, J = 8.5, 3.6 Hz, $1 \mathrm{H}), 4.25-4.01(\mathrm{~m}, 4 \mathrm{H}), 1.34(\mathrm{t}, \mathrm{J}=7.1 \mathrm{~Hz}, 6 \mathrm{H}) \mathrm{ppm}$.

${ }^{13} \mathrm{C}$ NMR $\left(101 \mathrm{MHz}, \mathrm{CDCl}_{3}\right) \delta 134.1(\mathrm{C}, \mathrm{d}, \mathrm{J}=187.8 \mathrm{~Hz}), 132.4(\mathrm{CH}, \mathrm{d}, \mathrm{J}=9.8 \mathrm{~Hz}), 132.1$ $(\mathrm{CH}, \mathrm{d}, \mathrm{J}=15.0 \mathrm{~Hz}), 118.0(\mathrm{C}, \mathrm{s}), 116.2(\mathrm{C}, \mathrm{d}, \mathrm{J}=3.6 \mathrm{~Hz}), 62.8\left(\mathrm{CH}_{2}, \mathrm{~d}, \mathrm{~J}=5.6 \mathrm{~Hz}\right)$, $16.5\left(\mathrm{CH}_{3}, \mathrm{~d}, \mathrm{~J}=6.3 \mathrm{~Hz}\right) \mathrm{ppm}$.

Spectral data were consistent with the literature. ${ }^{3}$

\section{5b. Diethyl (2-cyanophenyl)phosphonate}<smiles>CCOP(=O)(OCC)c1ccccc1C#N</smiles>

The compound (CAS: $34595-07-8$ ) was prepared according to the general procedure using 9-10-dicyanoanthracene $(1.2 \mathrm{mg}, 5 \mu \mathrm{mol}, 0.1$ equiv.), 2-bromobenzonitrile (9.2 $\mathrm{mg}, 50 \mu \mathrm{mol}, 1.0$ equiv.), triethylphosphite $(45 \mu \mathrm{L}, 250 \mu \mathrm{mol}, 5$ equiv.), dodecanenitrile $(12 \mu \mathrm{L}, 50$ $\mu \mathrm{mol}, 1.0$ equiv.) as internal standard, DIPEA (10.5 $\mu \mathrm{L}, 60 \mu \mathrm{mol}, 1.2$ equiv.) and $\mathrm{G} 1(10 \mathrm{mg} / \mathrm{mL})$. The reaction mixture was irradiated for 2 hours, obtaining $\mathbf{9 2 \%}$ product yield according to GC-FID analysis ( $83 \%$ isolated yield as colorless oil).

${ }^{1} \mathrm{H}$ NMR $\left(400 \mathrm{MHz}, \mathrm{CDCl}_{3}\right) \delta 8.16-8.07(\mathrm{~m}, 1 \mathrm{H}), 7.83-7.77(\mathrm{~m}, 1 \mathrm{H}), 7.74-7.61(\mathrm{~m}$, $2 \mathrm{H}), 4.39-4.03(\mathrm{~m}, 4 \mathrm{H}), 1.48-1.31(\mathrm{~m}, 6 \mathrm{H}) \mathrm{ppm}$.

${ }^{13} \mathrm{C}$ NMR $\left(101 \mathrm{MHz}, \mathrm{CDCl}_{3}\right) \delta 134.7(\mathrm{CH}, \mathrm{d}, \mathrm{J}=6.6 \mathrm{~Hz}), 134.6(\mathrm{CH}, \mathrm{d}, \mathrm{J}=9.2 \mathrm{~Hz}), 132.5$ $(\mathrm{CH}, \mathrm{d}, \mathrm{J}=2.6 \mathrm{~Hz}), 132.5(\mathrm{C}, \mathrm{d}, \mathrm{J}=188.0 \mathrm{~Hz}), 132.3(\mathrm{CH}, \mathrm{d}, \mathrm{J}=14.0 \mathrm{~Hz}), 117.2(\mathrm{C}, \mathrm{d}$, $\mathrm{J}=5.7 \mathrm{~Hz}), 114.8(\mathrm{C}, \mathrm{d}, \mathrm{J}=5.0 \mathrm{~Hz}), 63.3\left(\mathrm{CH}_{2}, \mathrm{~d}, \mathrm{~J}=6.0 \mathrm{~Hz}\right), 16.4\left(\mathrm{CH}_{3}, \mathrm{~d}, \mathrm{~J}=6.3 \mathrm{~Hz}\right)$ ppm.

Spectral data were consistent with the literature. ${ }^{4}$

\footnotetext{
${ }^{3}$ Rongqiang Zhuang, Jian Xu, Zhenshi Cai, Guo Tang, Meijuan Fang, and Yufen Zhao. Org. Lett. 2011, 13, 8, 2110-2113.

${ }^{4}$ I. Ghosh, R. S. Shaikh, B. König. Angew. Chem. Int. Ed. 2017, 56, 8544.

${ }^{5}$ Ya Bai, Nian Liu, Shutao Wang, Siyu Wang, Shulin Ning, Lingling Shi, Lili Cui, Zhuoqi Zhang, and Jinbao Xiang. Organic Letters 201921 (17), 6835-6838
} 
15c. diethyl quinolin-2-ylphosphonate<smiles>CCOP(=O)(OCC)c1ccc2ccccc2n1</smiles>

The compound (CAS: $37175-36-3)$ was prepared according to the general procedure using 9-10-dicyanoanthracene $(2.4 \mathrm{mg}, 10 \mu \mathrm{mol}$, 0.2 equiv.), 2-bromoquinoline (10.4 mg, $50 \mu \mathrm{mol}, 1.0$ equiv.), triethylphosphite $(180 \mu \mathrm{L}, 1000 \mu \mathrm{mol}, 20$ equiv.), dodecanenitrile (12 $\mu \mathrm{L}, 50 \mu \mathrm{mol}, 1.0$ equiv.) as internal standard, DIPEA $(10.5 \mu \mathrm{L}$, $60 \mu \mathrm{mol}, 1.2$ equiv.) and $\mathrm{G} 1(10 \mathrm{mg} / \mathrm{mL})$. The reaction mixture was irradiated for 10 hours, obtaining $89 \%$ product yield according to GC-FID analysis ( $74 \%$ isolated yield as colorless oil).

${ }^{1} \mathrm{H}$ NMR $\left(400 \mathrm{MHz}, \mathrm{CDCl}_{3}\right.$ ) $\delta 8.27$ (dd, J = 7.9, $6.3 \mathrm{~Hz}, 2 \mathrm{H}$ ), 8.00 (dd, J = 8.4, $4.7 \mathrm{~Hz}$, $1 \mathrm{H}), 7.87(\mathrm{dd}, \mathrm{J}=8.2,1.1 \mathrm{~Hz}, 1 \mathrm{H}), 7.78(\mathrm{ddd}, \mathrm{J}=8.5,6.9,1.4 \mathrm{~Hz}, 1 \mathrm{H}), 7.64(\mathrm{dd}, \mathrm{J}=$ $11.1,4.1 \mathrm{~Hz}, 1 \mathrm{H}), 4.39-4.22(\mathrm{~m}, 4 \mathrm{H}), 1.38(\mathrm{t}, \mathrm{J}=7.1 \mathrm{~Hz}, 6 \mathrm{H}) \mathrm{ppm}$.

Spectral data were consistent with the literature. ${ }^{5}$

\section{5d. Diethyl quinolin-3-ylphosphonate}<smiles>CCOP(=O)(OCC)c1cnc2ccccc2c1</smiles>

The compound (CAS: 82594-50-1) was prepared according to the general procedure using 9-10-dicyanoanthracene $(2.4 \mathrm{mg}, 10 \mu \mathrm{mol}$, 0.2 equiv.), 3-bromoquinoline (6.9 $\mu \mathrm{L}, 50 \mu \mathrm{mol}, 1.0$ equiv.), triethylphosphite $(180 \mu \mathrm{L}, 1000 \mu \mathrm{mol}, 20$ equiv.), dodecanenitrile (12 $\mu \mathrm{L}, 50 \mu \mathrm{mol}, 1.0$ equiv.) as internal standard, DIPEA (10.5 $\mu \mathrm{L}, 60 \mu \mathrm{mol}, 1.2$ equiv.) and $\mathrm{G} 1(10 \mathrm{mg} / \mathrm{mL})$. The reaction mixture was irradiated for 10 hours, obtaining $\mathbf{8 3} \%$ product yield according to GC-FID analysis (69\% isolated yield as pale-yellow oil).

${ }^{1} \mathrm{H}$ NMR $\left(400 \mathrm{MHz}, \mathrm{CDCl}_{3}\right) \delta 9.15$ (dd, J = 4.5, 2.0 Hz, $\left.1 \mathrm{H}\right), 8.71$ (dd, J = 15.2, $1.3 \mathrm{~Hz}$, $1 \mathrm{H}), 8.16(\mathrm{~d}, \mathrm{~J}=8.0 \mathrm{~Hz}, 1 \mathrm{H}), 7.92(\mathrm{dd}, J=8.2,0.9 \mathrm{~Hz}, 1 \mathrm{H}), 7.85(\mathrm{ddd}, \mathrm{J}=8.4,7.0,1.3$ $\mathrm{Hz}, 1 \mathrm{H}), 7.64(\mathrm{ddd}, \mathrm{J}=8.1,7.0,1.0 \mathrm{~Hz}, 1 \mathrm{H}), 4.37-4.06(\mathrm{~m}, 4 \mathrm{H}), 1.36(\mathrm{td}, \mathrm{J}=7.1,0.4$ $\mathrm{Hz}, 6 \mathrm{H}) \mathrm{ppm}$.

${ }^{13} \mathrm{C}$ NMR (101 MHz, CDCl 3 ) $\delta 150.7(\mathrm{CH}, \mathrm{d}, \mathrm{J}=12.2 \mathrm{~Hz}), 149.5(\mathrm{C}, \mathrm{d}, \mathrm{J}=1.2 \mathrm{~Hz}), 142.2$ $(\mathrm{CH}, \mathrm{d}, \mathrm{J}=8.6 \mathrm{~Hz}), 132.0(\mathrm{CH}, \mathrm{s}), 129.7(\mathrm{CH}, \mathrm{d}, \mathrm{J}=1.1 \mathrm{~Hz}), 128.8(\mathrm{CH}, \mathrm{s}), 127.8(\mathrm{CH}$, $\mathrm{d}, \mathrm{J}=1.1 \mathrm{~Hz}), 126.9(\mathrm{C}, \mathrm{d}, \mathrm{J}=13.5 \mathrm{~Hz}), 122.1(\mathrm{C}, \mathrm{d}, \mathrm{J}=189.3 \mathrm{~Hz}), 62.8\left(\mathrm{CH}_{2}, \mathrm{~d}, \mathrm{~J}=5.5\right.$ $\mathrm{Hz}), 16.5\left(\mathrm{CH}_{3}, \mathrm{~d}, \mathrm{~J}=6.4 \mathrm{~Hz}\right) \mathrm{ppm}$.

Spectral data were consistent with the literature. ${ }^{4}$

\section{5e. Tert-butyl 2-(diethoxyphosphoryl)-3-methyl-1H-indole-1-carboxylate}<smiles>CCOP(=O)(OCC)c1[nH]c2ccccc2c1C</smiles>

The compound (CAS: 2035049-93-3) was prepared according to the general procedure using 9-10-dicyanoanthracene $(1.2 \mathrm{mg}, 5$ umol, 0.1 equiv.), $\mathrm{N}$-Boc-2-bromo-3-methylindole $(16.0 \mathrm{mg}, 50$ $\mu \mathrm{mol}, 1.0$ equiv.), triethylphosphite $(45 \mu \mathrm{L}, 250 \mu \mathrm{mol}, 5$ equiv.), dodecanenitrile (12 $\mu \mathrm{L}, 50 \mu \mathrm{mol}, 1.0$ equiv.) as internal standard, DIPEA (10.5 $\mu \mathrm{L}, 60 \mu \mathrm{mol}, 1.2$ equiv.) and $\mathrm{G} 1(10 \mathrm{mg} / \mathrm{mL})$. The reaction mixture was irradiated for 16 hours, obtaining $\mathbf{8 1} \%$ product yield according to GC-FID analysis (65\% isolated yield as yellow oil).

${ }^{1} \mathrm{H}$ NMR $\left(400 \mathrm{MHz}, \mathrm{CDCl}_{3}\right) \delta 8.07(\mathrm{~d}, \mathrm{~J}=8.5 \mathrm{~Hz}, 1 \mathrm{H}), 7.61(\mathrm{~d}, \mathrm{~J}=7.8 \mathrm{~Hz}, 1 \mathrm{H}), 7.42$ (dd, $\mathrm{J}=8.2,7.4 \mathrm{~Hz}, 1 \mathrm{H}), 7.30-7.25(\mathrm{~m}, 1 \mathrm{H}), 4.32-4.01(\mathrm{~m}, 4 \mathrm{H}), 2.60(\mathrm{~d}, \mathrm{~J}=2.2 \mathrm{~Hz}, 3 \mathrm{H})$, $1.69(\mathrm{~s}, 9 \mathrm{H}), 1.38-1.33(\mathrm{~m}, 6 \mathrm{H}) \mathrm{ppm}$.

${ }^{13} \mathrm{C}$ NMR (101 MHz, $\left.\mathrm{CDCl}_{3}\right) \delta 149.9(\mathrm{C}, \mathrm{s}), 138.1$ (C, d, J = 8.8 Hz), 132.1 (C, d, J = 16.9 $\mathrm{Hz}), 130.0$ (C, d, J = 17.0 Hz), $127.3(\mathrm{CH}, \mathrm{s}), 122.8(\mathrm{CH}, \mathrm{s}), 122.3(\mathrm{C}, \mathrm{d}, \mathrm{J}=220.5 \mathrm{~Hz})$, 
$120.2(\mathrm{CH}, \mathrm{s}), 115.4(\mathrm{CH}, \mathrm{s}), 84.9(\mathrm{C}, \mathrm{s}), 62.5\left(\mathrm{CH}_{2}, \mathrm{~d}, \mathrm{~J}=5.5 \mathrm{~Hz}\right), 28.2\left(\mathrm{CH}_{3}, \mathrm{~s}\right), 16.5$ $\left(\mathrm{CH}_{3}, \mathrm{~d}, \mathrm{~J}=6.8 \mathrm{~Hz}\right), 11.1\left(\mathrm{CH}_{3}, \mathrm{~s}\right) \mathrm{ppm}$.

Spectral data were consistent with the literature. ${ }^{6}$

\section{RADICAL TRAPPING}

\section{Tert-butyl 2-(diethoxyphosphoryl)-3-methyl-1H-indole-1-carboxylate}<smiles>N#Cc1ccc([SbH]c2ccccc2)s1</smiles>

The compound (CAS: 1300106-13-1) was prepared according to the general procedure using 9-10-dicyanoanthracene $(1.2 \mathrm{mg}, 5 \mu \mathrm{mol}, 0.1$ equiv.), $N$-Boc-2-bromo-3-methylindole ( $16.0 \mathrm{mg}, 50 \mu \mathrm{mol}, 1.0$ equiv.), triethylphosphite $(45 \mu \mathrm{L}, 250 \mu \mathrm{mol}, 5$ equiv.), diphenyl disulfide $(47.5 \mathrm{mg}, 250 \mu \mathrm{mol}, 5$ equiv.) as trapping agent, dodecanenitrile $(12 \mu \mathrm{L}, 50 \mu \mathrm{mol}, 1.0$ equiv.) as internal standard, DIPEA $(10.5 \mu \mathrm{L}, 60 \mu \mathrm{mol}, 1.2$ equiv.) and $\mathrm{G} 1(10 \mathrm{mg} / \mathrm{mL})$. The reaction mixture was irradiated for 2.5 hours, obtaining $56 \%$ product yield according to GC-FID analysis ( $41 \%$ isolated yield as colorless oil).

${ }^{1} \mathrm{H}$ NMR $\left(400 \mathrm{MHz}, \mathrm{CDCl}_{3}\right) \delta 7.50(\mathrm{~d}, \mathrm{~J}=3.9 \mathrm{~Hz}, 1 \mathrm{H}), 7.41-7.31(\mathrm{~m}, 5 \mathrm{H}), 7.10(\mathrm{~d}, \mathrm{~J}=$ $3.9 \mathrm{~Hz}, 1 \mathrm{H}) \mathrm{ppm}$.

${ }^{13} \mathrm{C}$ NMR (101 MHz, $\left.\mathrm{CDCl}_{3}\right) \delta 144.4(\mathrm{C}, \mathrm{s}), 137.9(\mathrm{CH}, \mathrm{s}), 134.8(\mathrm{C}, \mathrm{s}), 131.9(\mathrm{CH}, \mathrm{s})$, $130.8(\mathrm{CH}, \mathrm{s}), 129.8(\mathrm{CH}, \mathrm{s}), 128.5(\mathrm{CH}, \mathrm{s}), 113.7(\mathrm{C}, \mathrm{s}), 112.1(\mathrm{C}, \mathrm{s}) \mathrm{ppm}$.

HRMS (EI): $\mathrm{m} / \mathrm{z}(\mathrm{M}+\mathrm{H})^{+}=$calcd. for $\mathrm{C}_{11} \mathrm{H}_{7} \mathrm{NS}_{2}: 218.0093$, found: 218.0089 .

Spectral data were consistent with the literature. ${ }^{7}$

\footnotetext{
${ }^{4}$ I. Ghosh, R. S. Shaikh, B. König. Angew. Chem. Int. Ed. 2017, 56, 8544.

${ }^{6}$ Shaikh, Rizwan S.; Duesel, Simon J. S.; Koenig, Burkhard. ACS Catalysis 2016, 6(12), 8410-8414.

${ }^{7}$ Mieko Arisawaa, Fumihiko Toriyama, Masahiko Yamaguchi. Tetrahedron Letters 52, 18, 2344-2347.
} 
Thiophene phosphonates

\section{NMR Spectra}

${ }^{1} \mathrm{H} 400 \mathrm{MHz}, \mathrm{CDCl}_{3}$

$3 a$

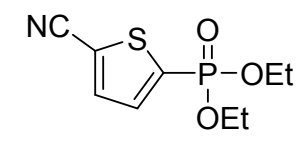

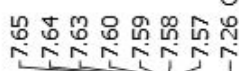

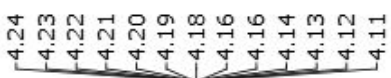

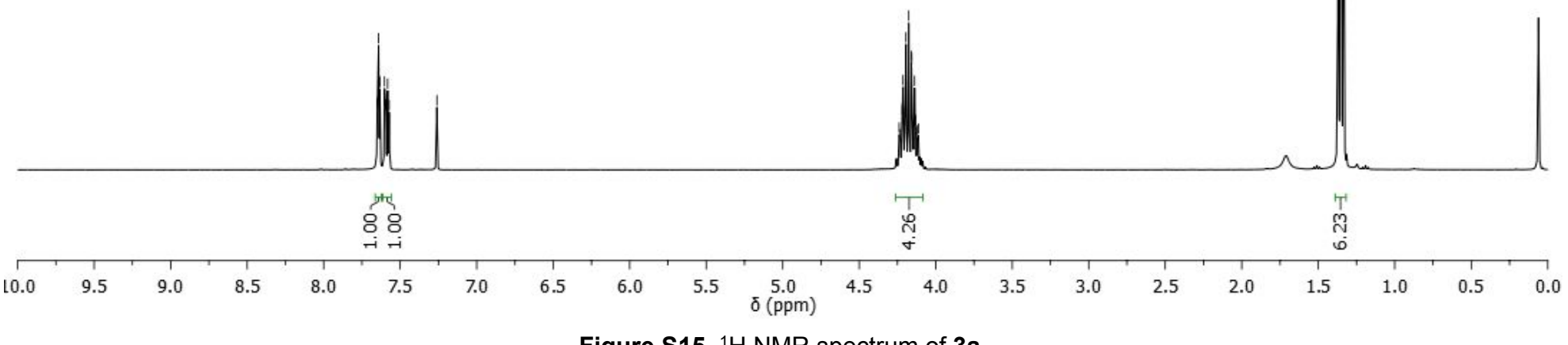

Figure S15. ${ }^{1} \mathrm{H}$ NMR spectrum of $\mathbf{3 a}$. 
Thiophene phosphonates

${ }^{13} \mathrm{C} 101 \mathrm{MHz}, \mathrm{CDCl}_{3}$

$3 a$
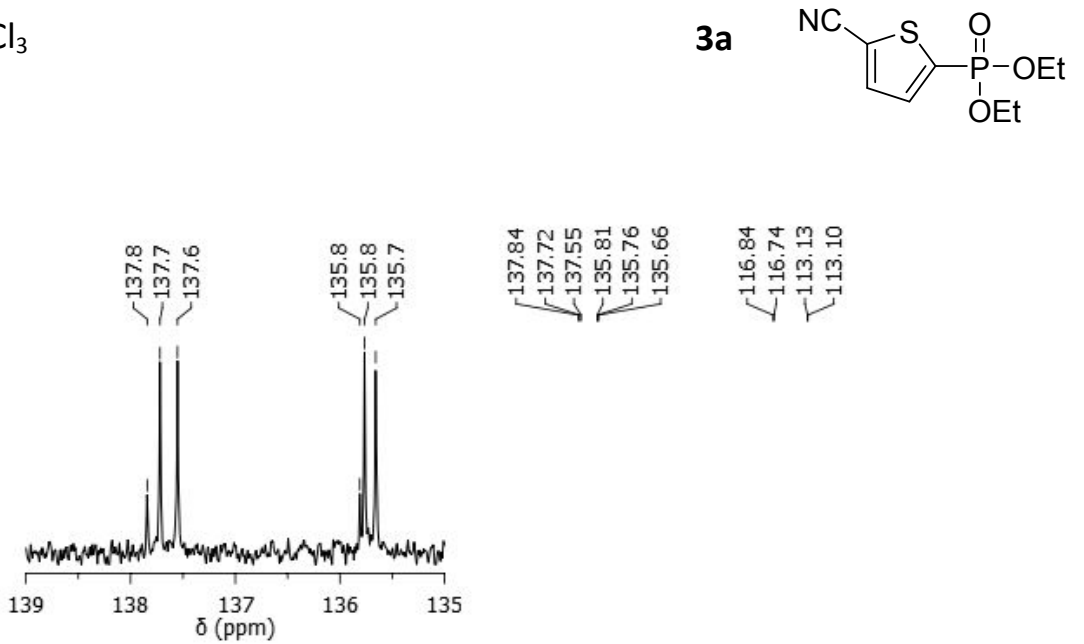

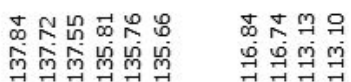

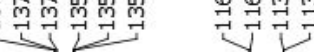

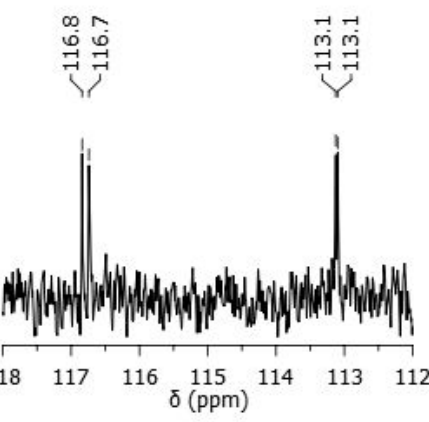

(n)

$\begin{array}{cccc}115 & 114 & 113 & 112\end{array}$

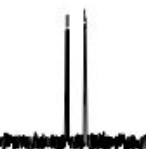

$200-190-180-170$

140

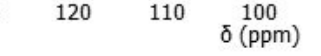

Figure S16. ${ }^{13} \mathrm{C}$ NMR spectrum of $\mathbf{3 a}$ 
Thiophene phosphonates

${ }^{31} \mathrm{P} 162 \mathrm{MHz}, \mathrm{CDCl}_{3}$

$3 a$
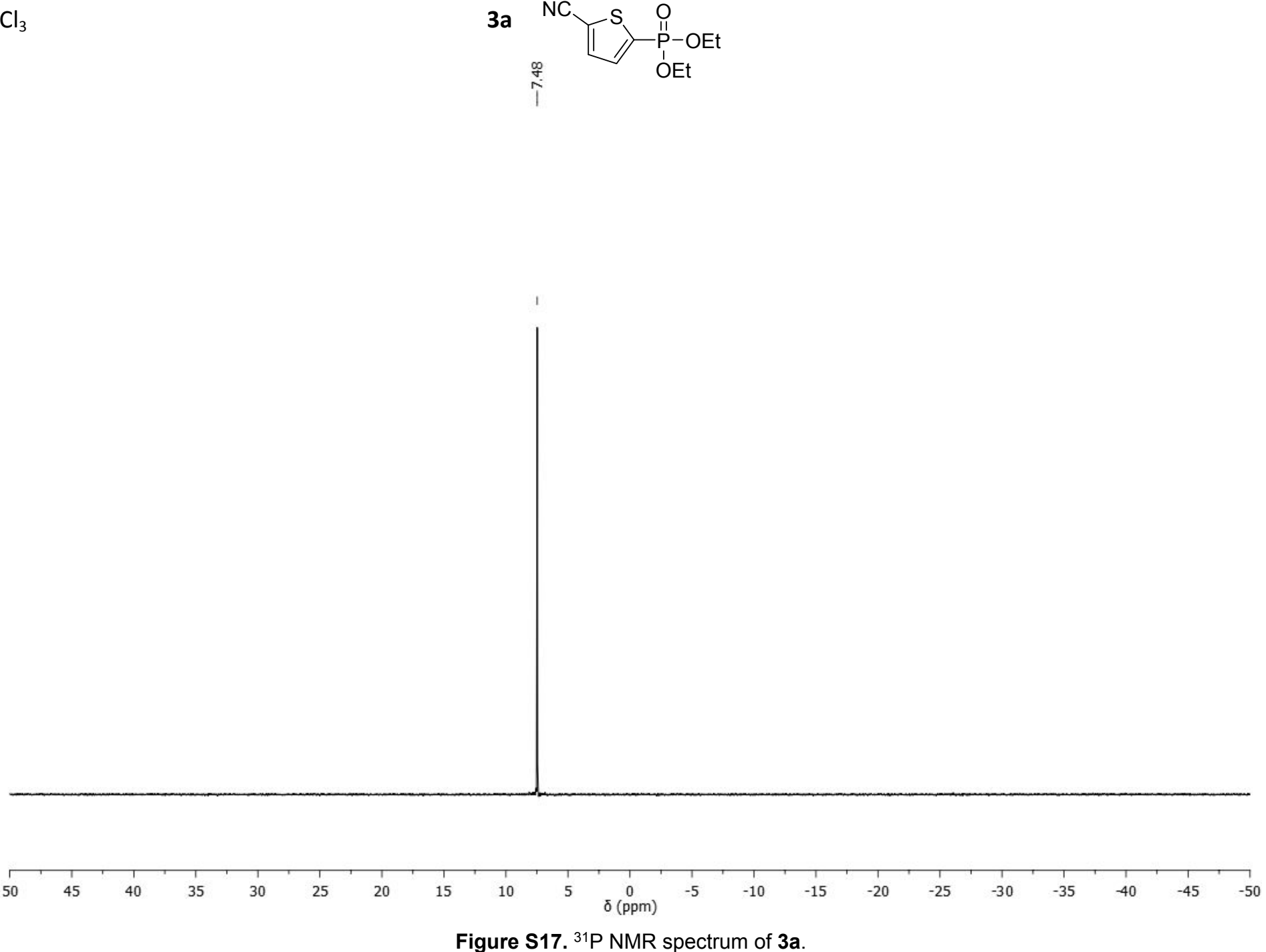
Thiophene phosphonates

${ }^{1} \mathrm{H} 400 \mathrm{MHz}, \mathrm{CDCl}_{3}$

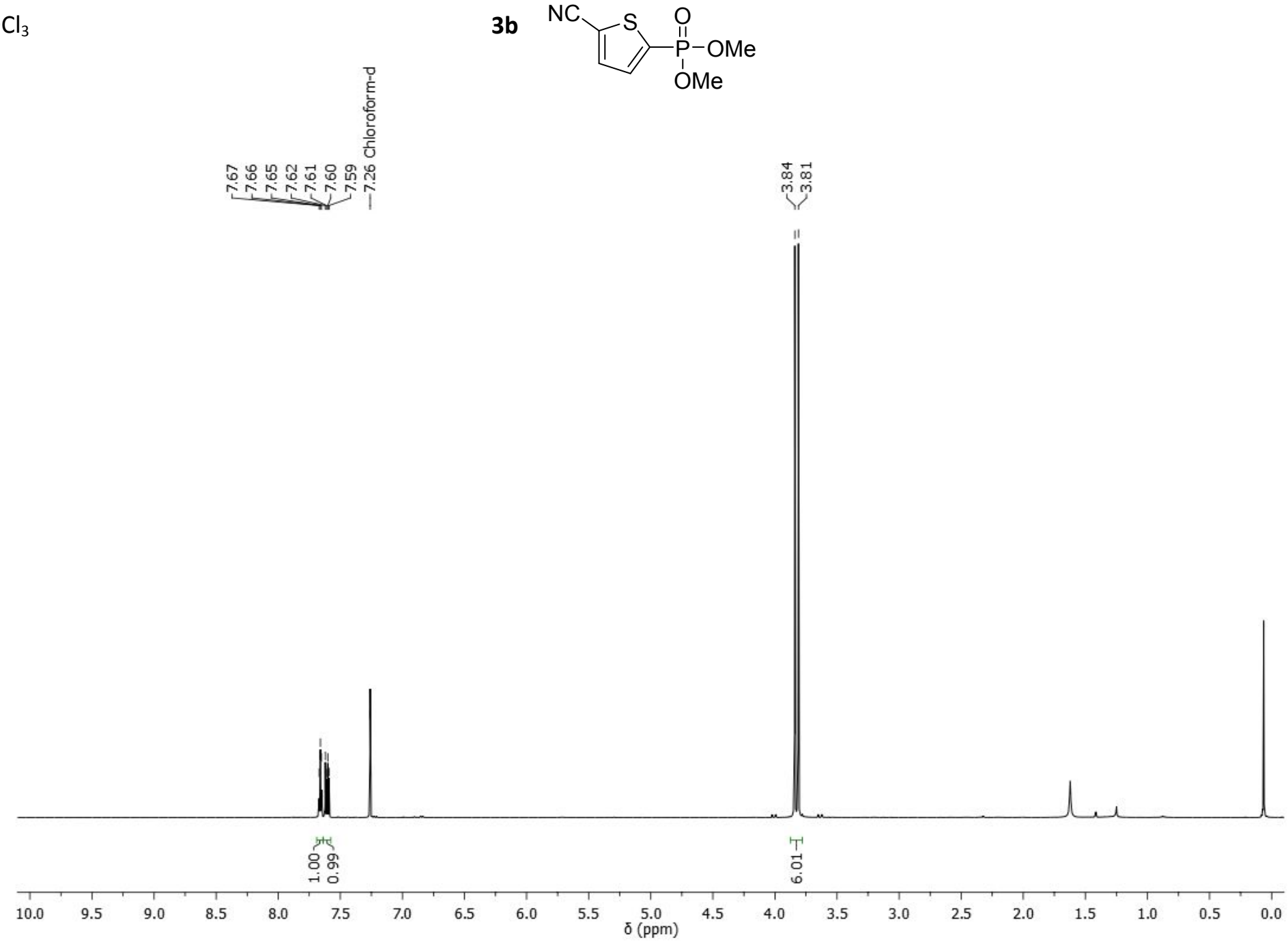

Figure S18. ${ }^{1} \mathrm{H}$ NMR spectrum of $\mathbf{3 b}$. 
Thiophene phosphonates

${ }^{13} \mathrm{C} 101 \mathrm{MHz}, \mathrm{CDCl}_{3}$

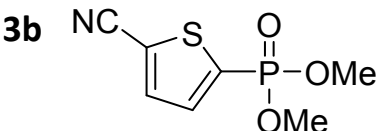

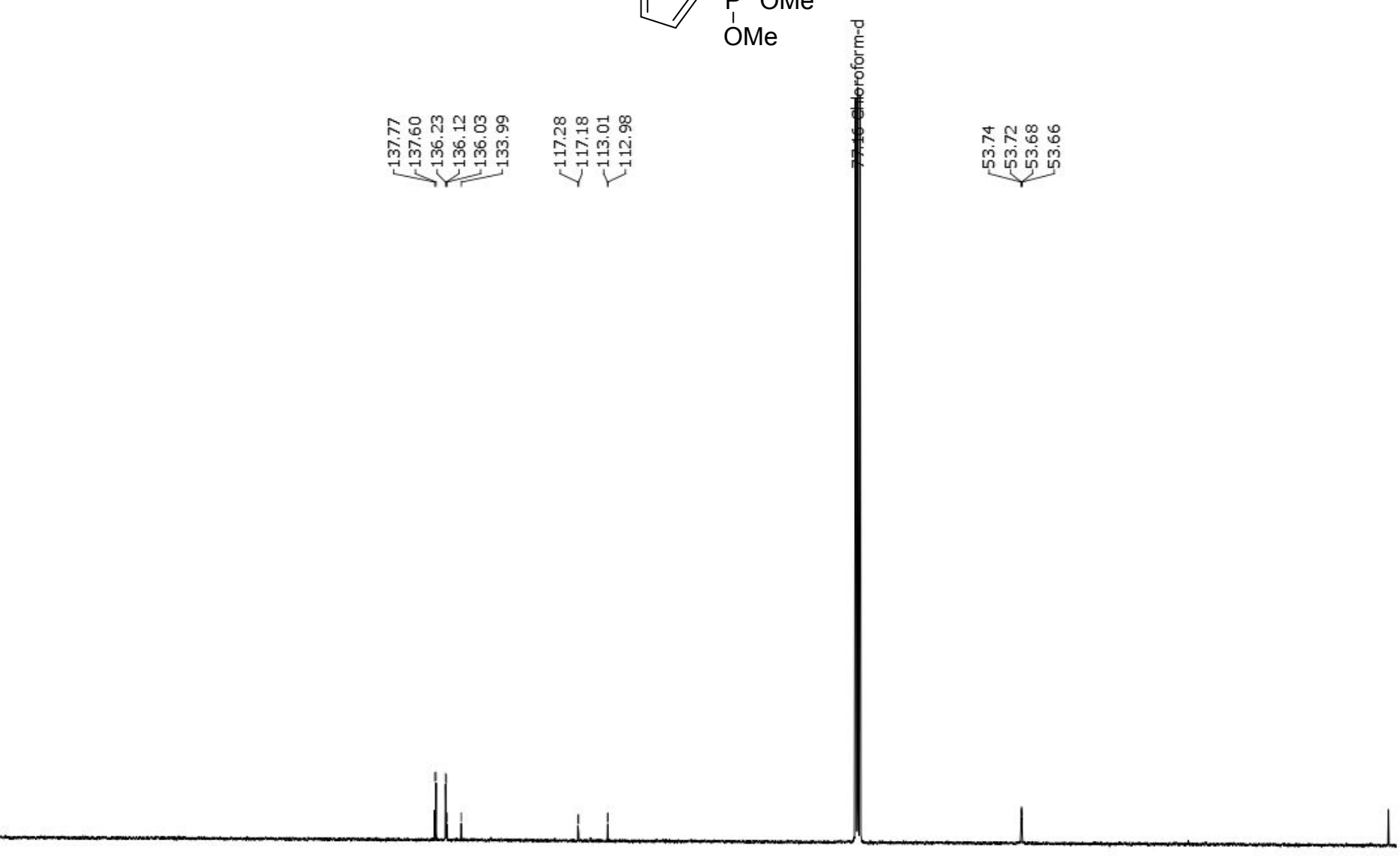

$\begin{array}{llllllll}200 & 190 & 180 & 170 & 160 & 150 & 140 & 130\end{array}$

Figure $\mathbf{S} 19 .{ }^{13} \mathrm{C}$ NMR spectrum of $\mathbf{3 b}$ 
${ }^{31} \mathrm{P} 162 \mathrm{MHz}, \mathrm{CDCl}_{3}$
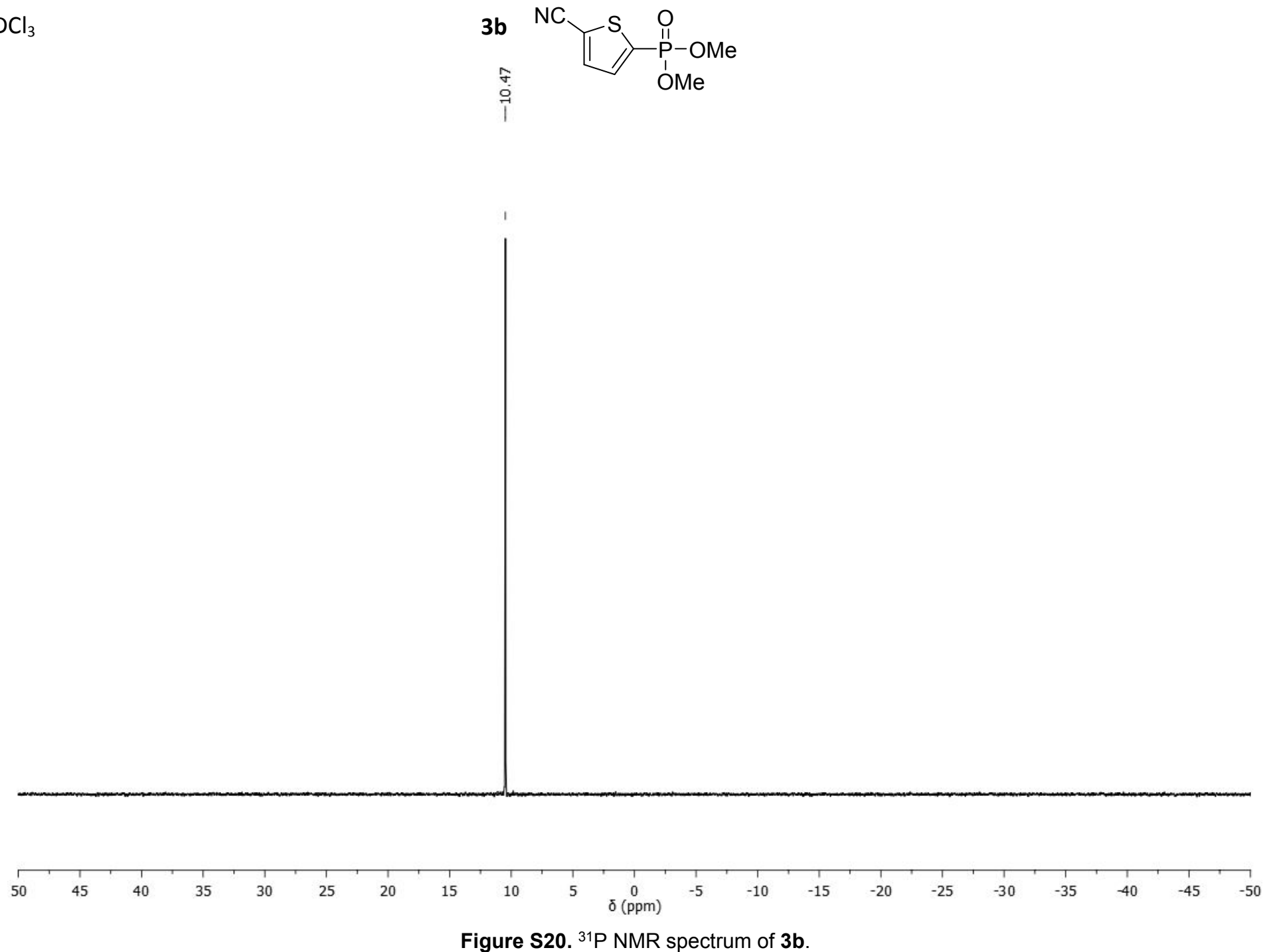
Thiophene phosphonates

${ }^{1} \mathrm{H} 400 \mathrm{MHz}, \mathrm{CDCl}_{3}$

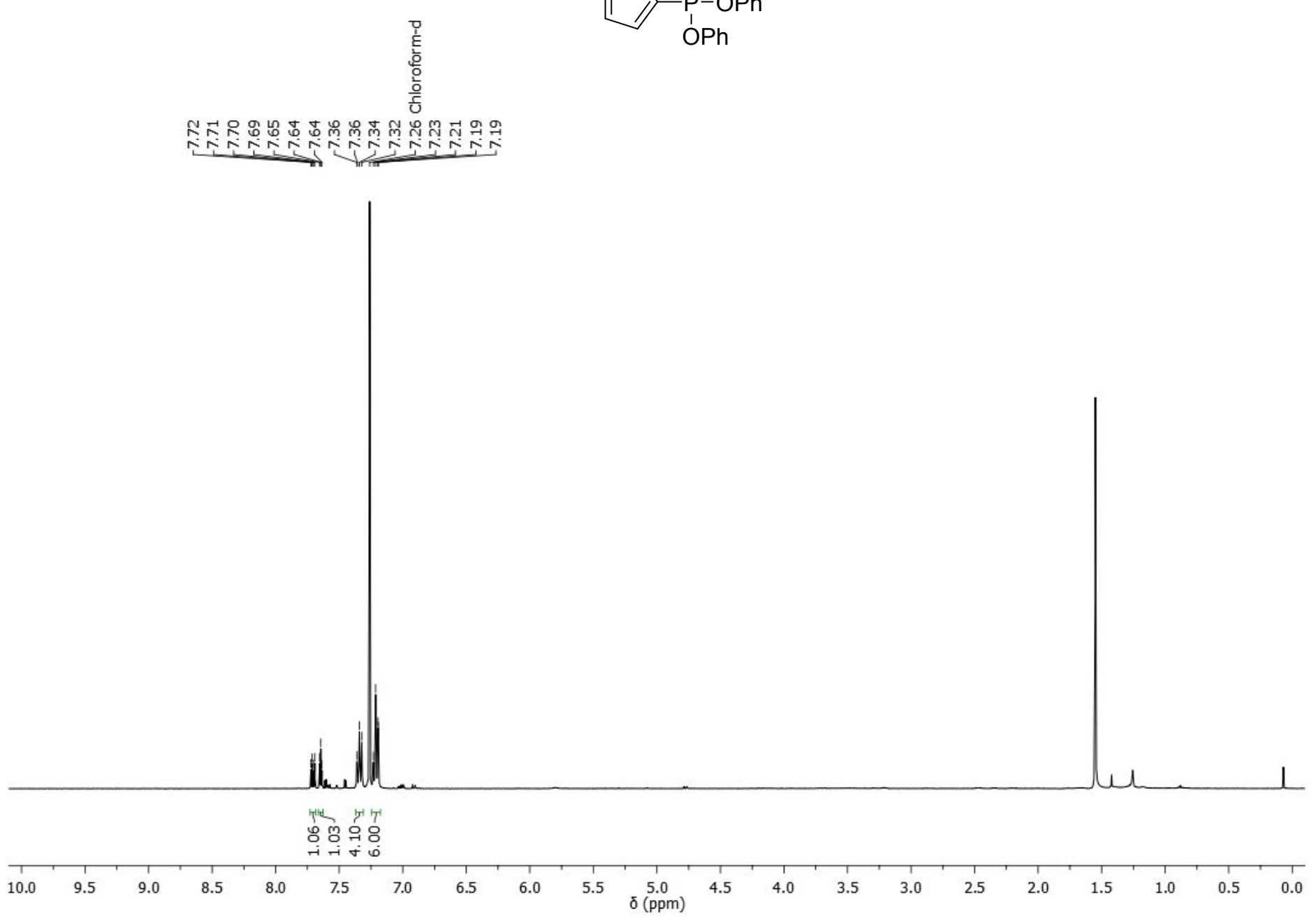

Figure S21. ${ }^{1} \mathrm{H}$ NMR spectrum of 3c.

S42 
Thiophene phosphonates

${ }^{13} \mathrm{C} 101 \mathrm{MHz}, \mathrm{CDCl}_{3}$

3c

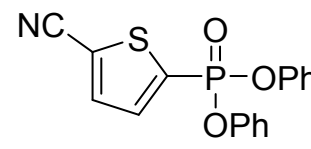

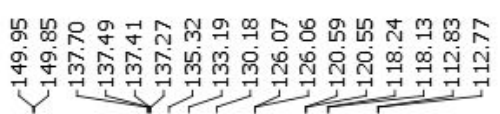

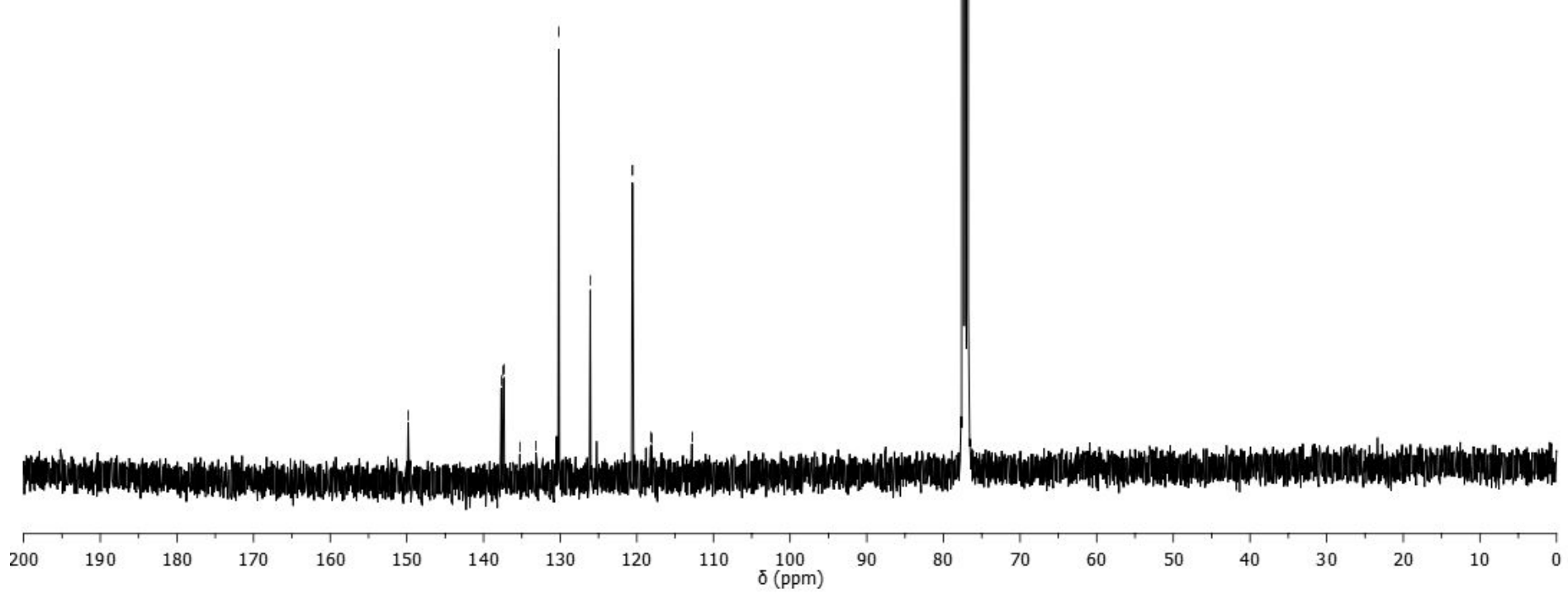

Figure S22. ${ }^{13} \mathrm{C}$ NMR spectrum of 3c.

S43 
${ }^{31} \mathrm{P} 162 \mathrm{MHz}, \mathrm{CDCl}_{3}$

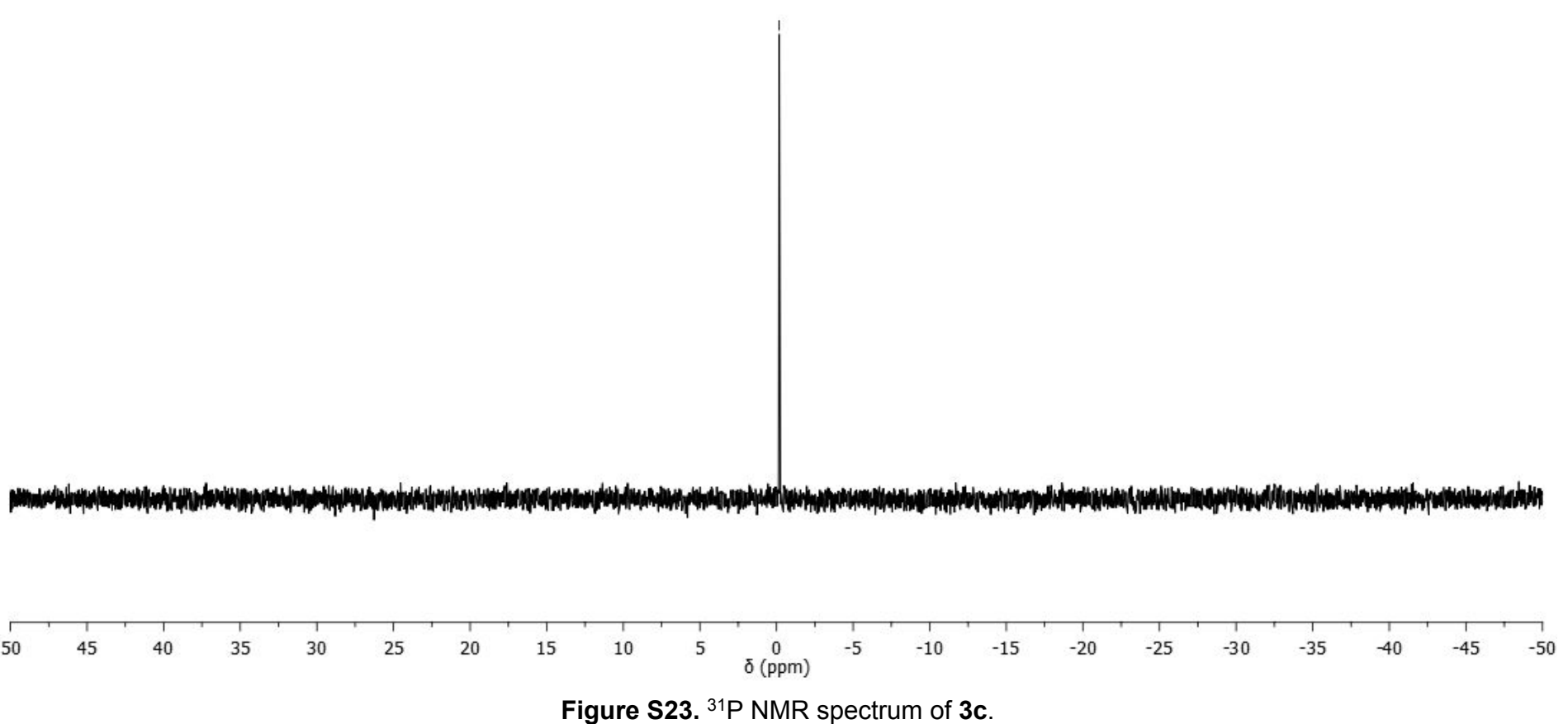


Thiophene phosphonates

${ }^{1} \mathrm{H} 400 \mathrm{MHz}, \mathrm{CDCl}_{3}$
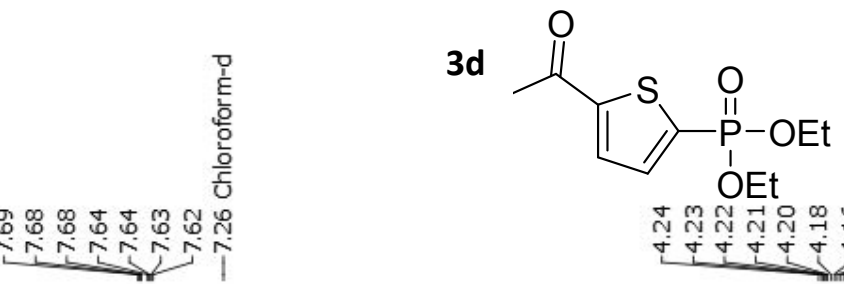

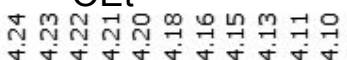
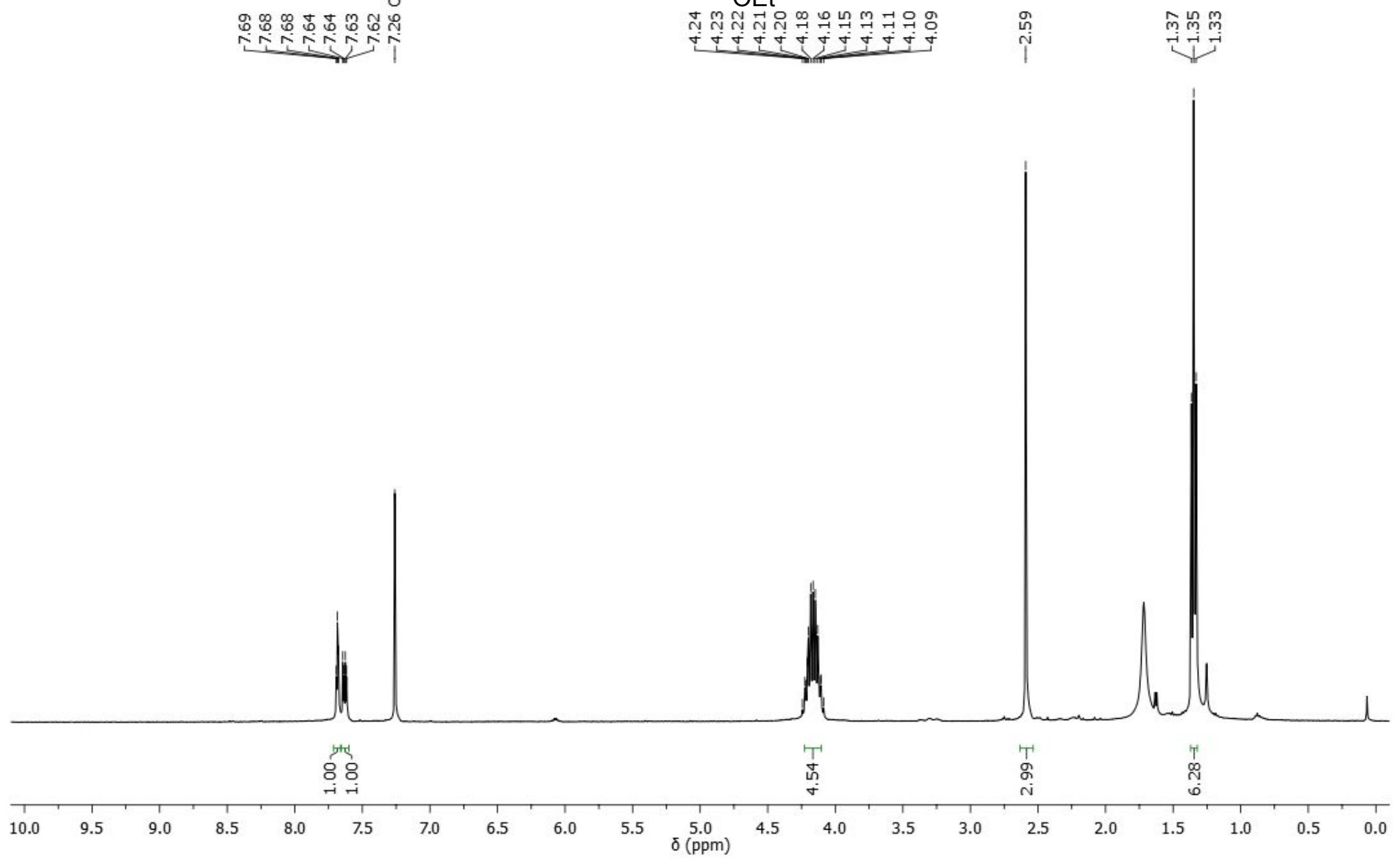

Figure S24. ${ }^{1} \mathrm{H}$ NMR spectrum of $\mathbf{3 d}$. 
Thiophene phosphonates

${ }^{13} \mathrm{C} 101 \mathrm{MHz}, \mathrm{CDCl}_{3}$

3d

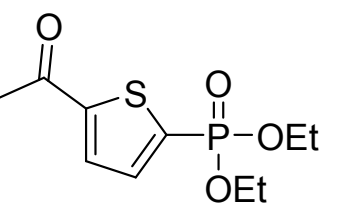

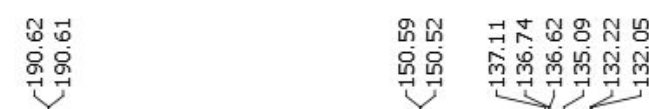

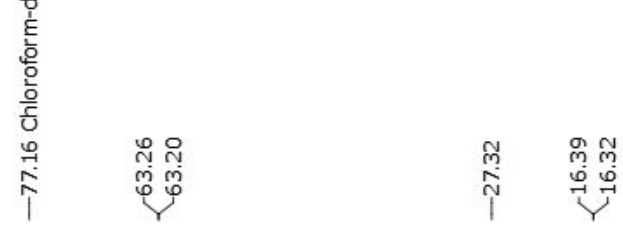

Figure S25. ${ }^{13} \mathrm{C}$ NMR spectrum of $\mathbf{3 d}$. 
${ }^{31} \mathrm{P} 162 \mathrm{MHz}, \mathrm{CDCl}_{3}$
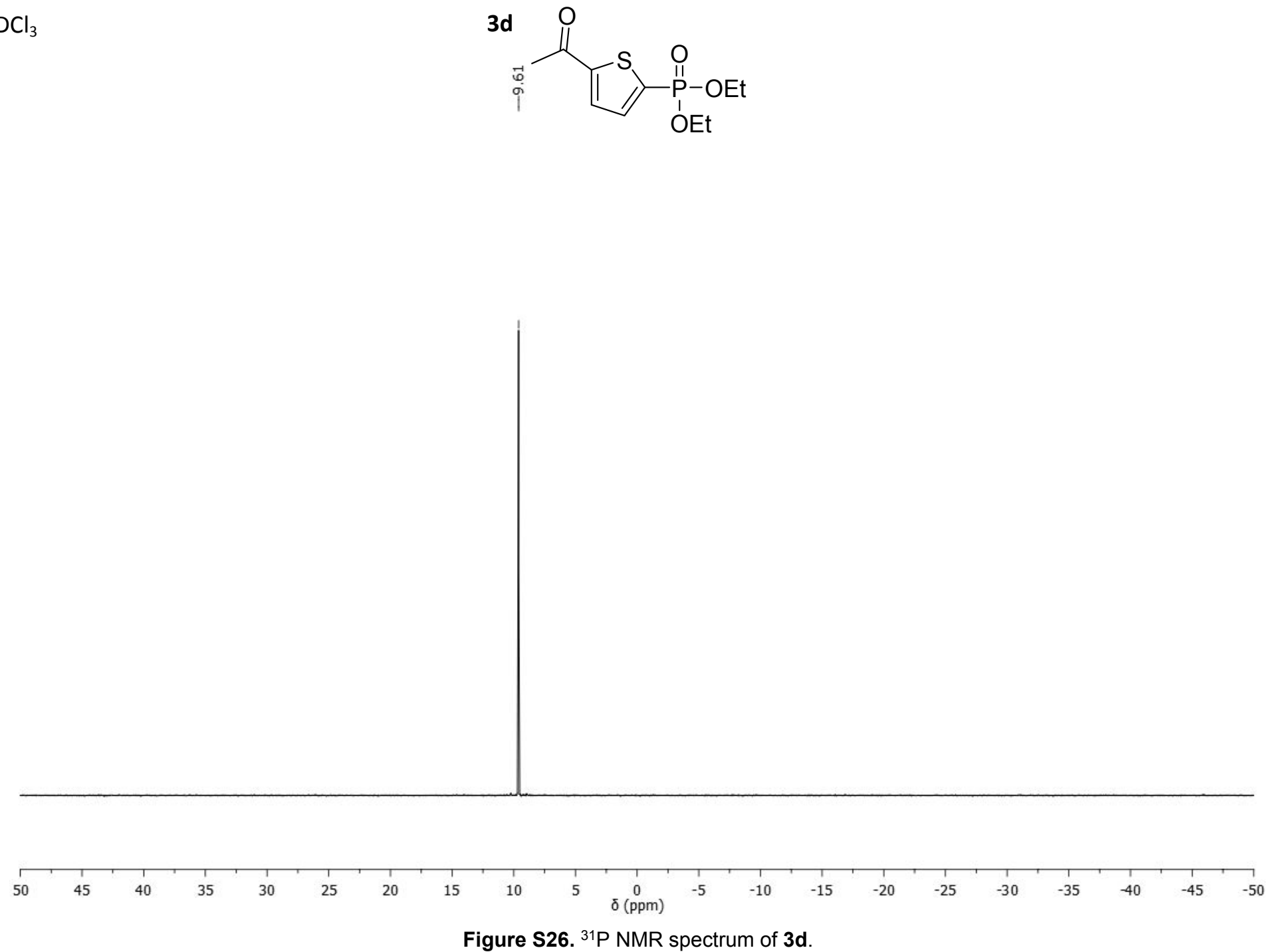
Thiophene phosphonates

${ }^{1} \mathrm{H} 400 \mathrm{MHz}, \mathrm{CDCl}_{3}$

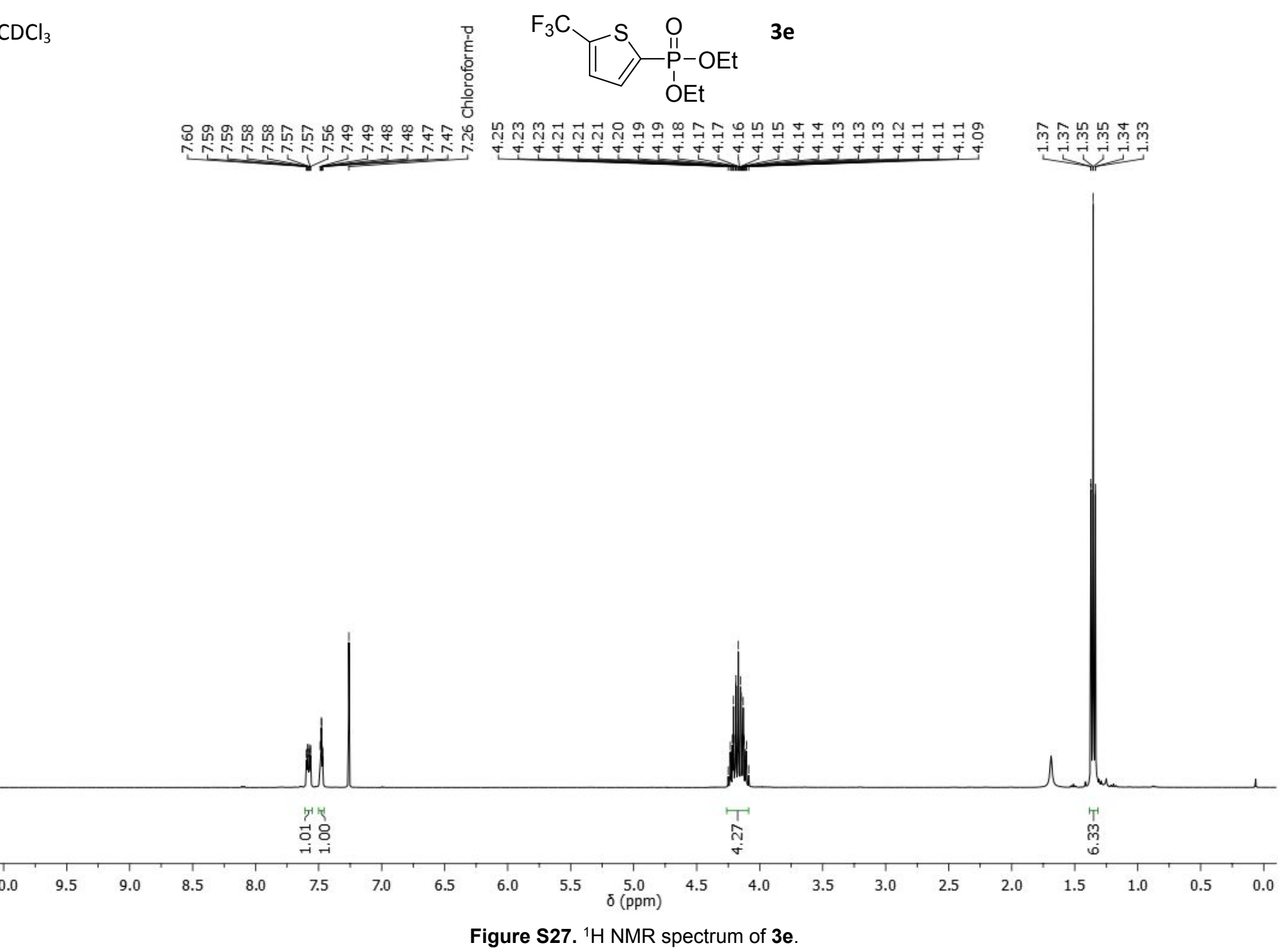


Thiophene phosphonates

${ }^{13} \mathrm{C} 101 \mathrm{MHz}, \mathrm{CDCl}_{3}$

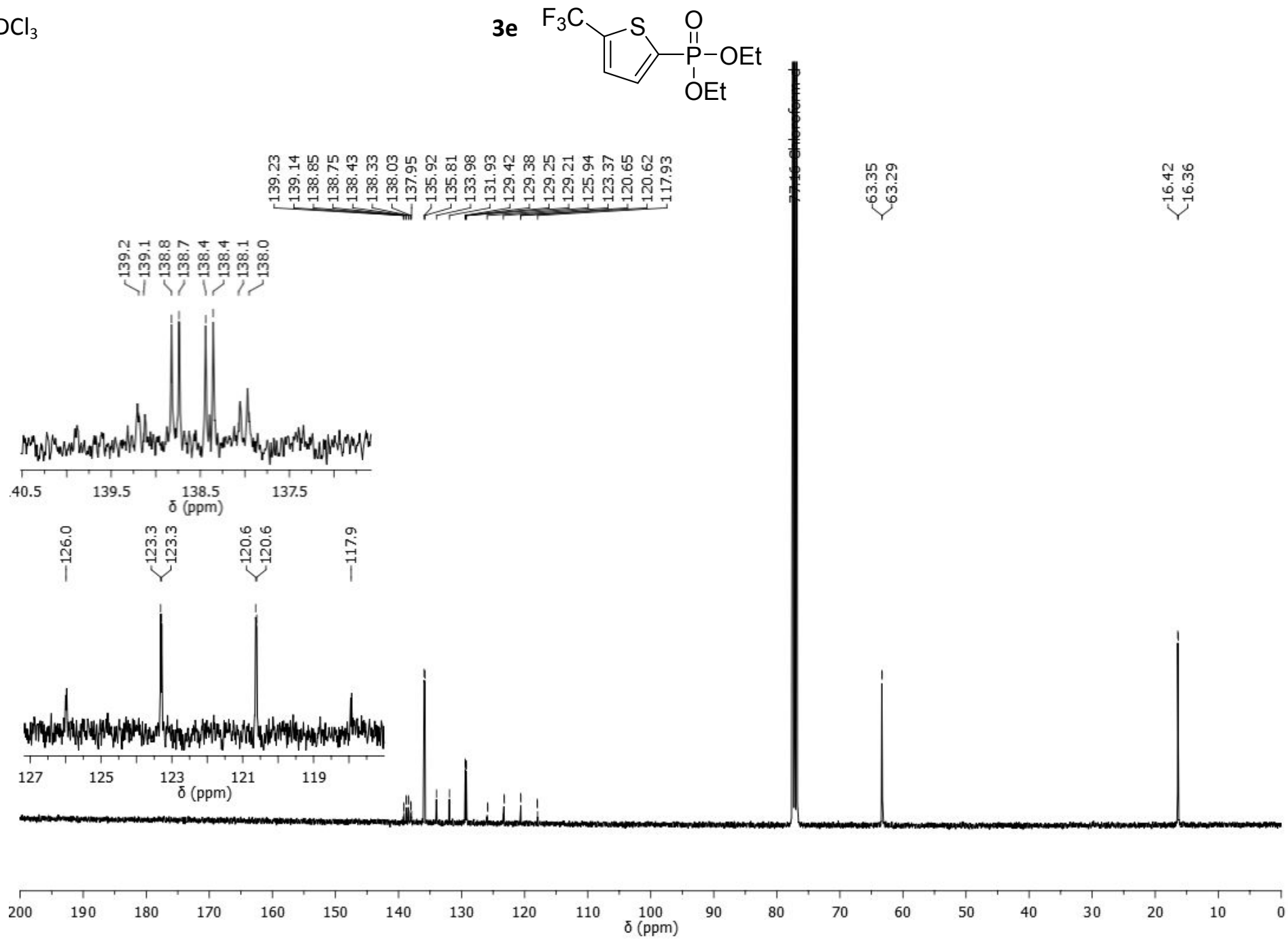

Figure S28. ${ }^{13} \mathrm{C}$ NMR spectrum of $\mathbf{3 e}$. 
${ }^{19} \mathrm{~F} 376 \mathrm{MHz} \mathrm{CDCl}_{3}$

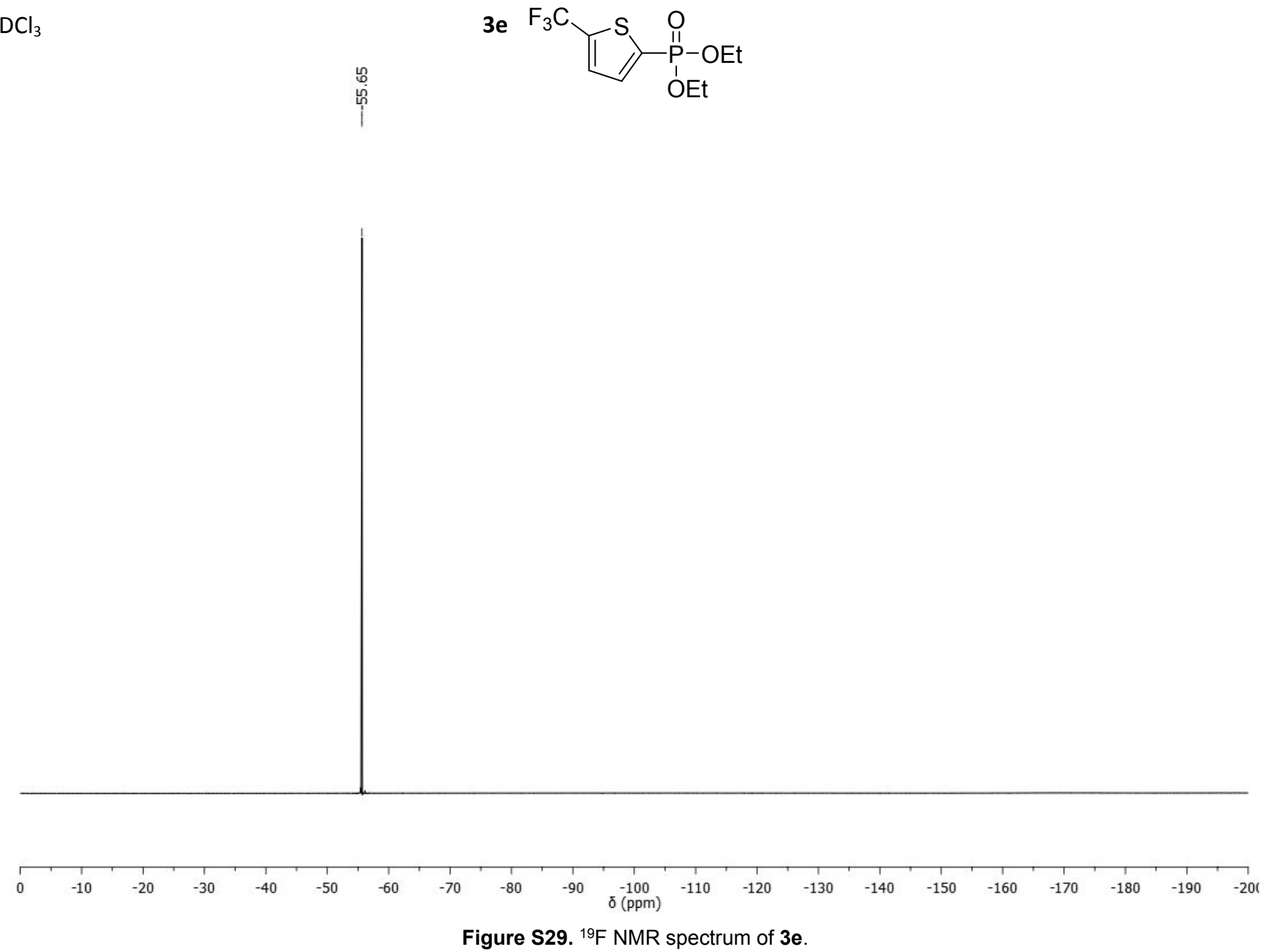


${ }^{31} \mathrm{P} 162 \mathrm{MHz}, \mathrm{CDCl}_{3}$

$3 e$

$$
\stackrel{\substack{O \\ \text { OEt }}}{\mathrm{P}_{3} \mathrm{C}-\mathrm{OEt}}
$$

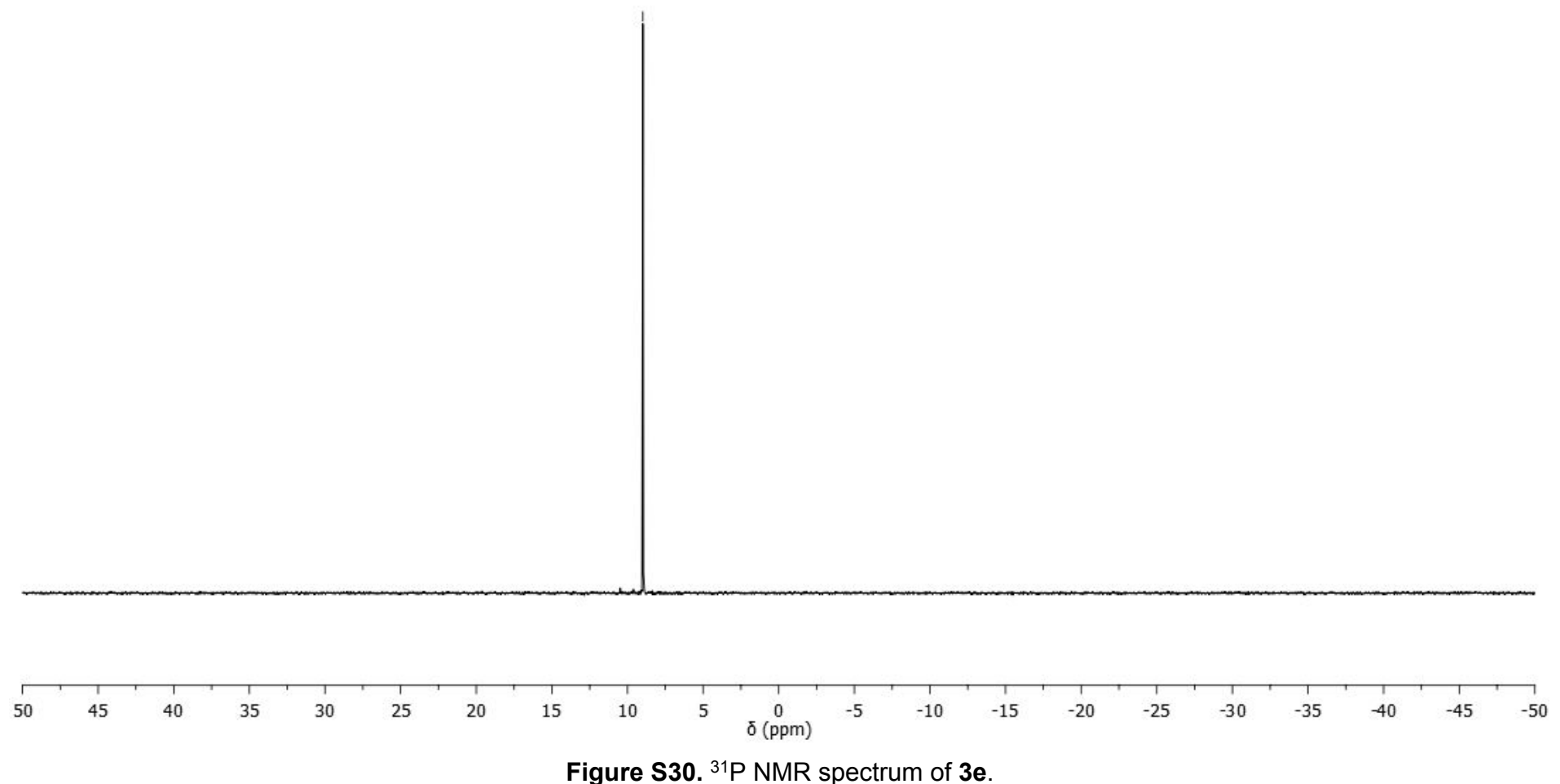


Thiophene phosphonates

${ }^{1} \mathrm{H} 400 \mathrm{MHz}, \mathrm{CDCl}_{3}$

$3 f \stackrel{\mathrm{H}_{3} \mathrm{C}}{\amalg S} \underset{\substack{\mathrm{O} \\ \mathrm{I} \\ \mathrm{O}-\mathrm{OEt}}}{\mathrm{O}}$

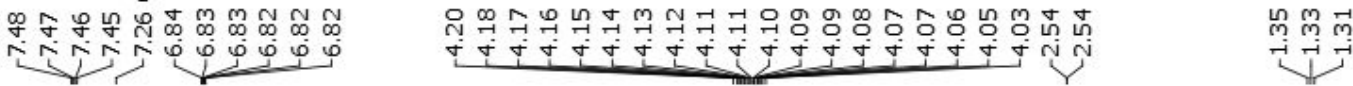

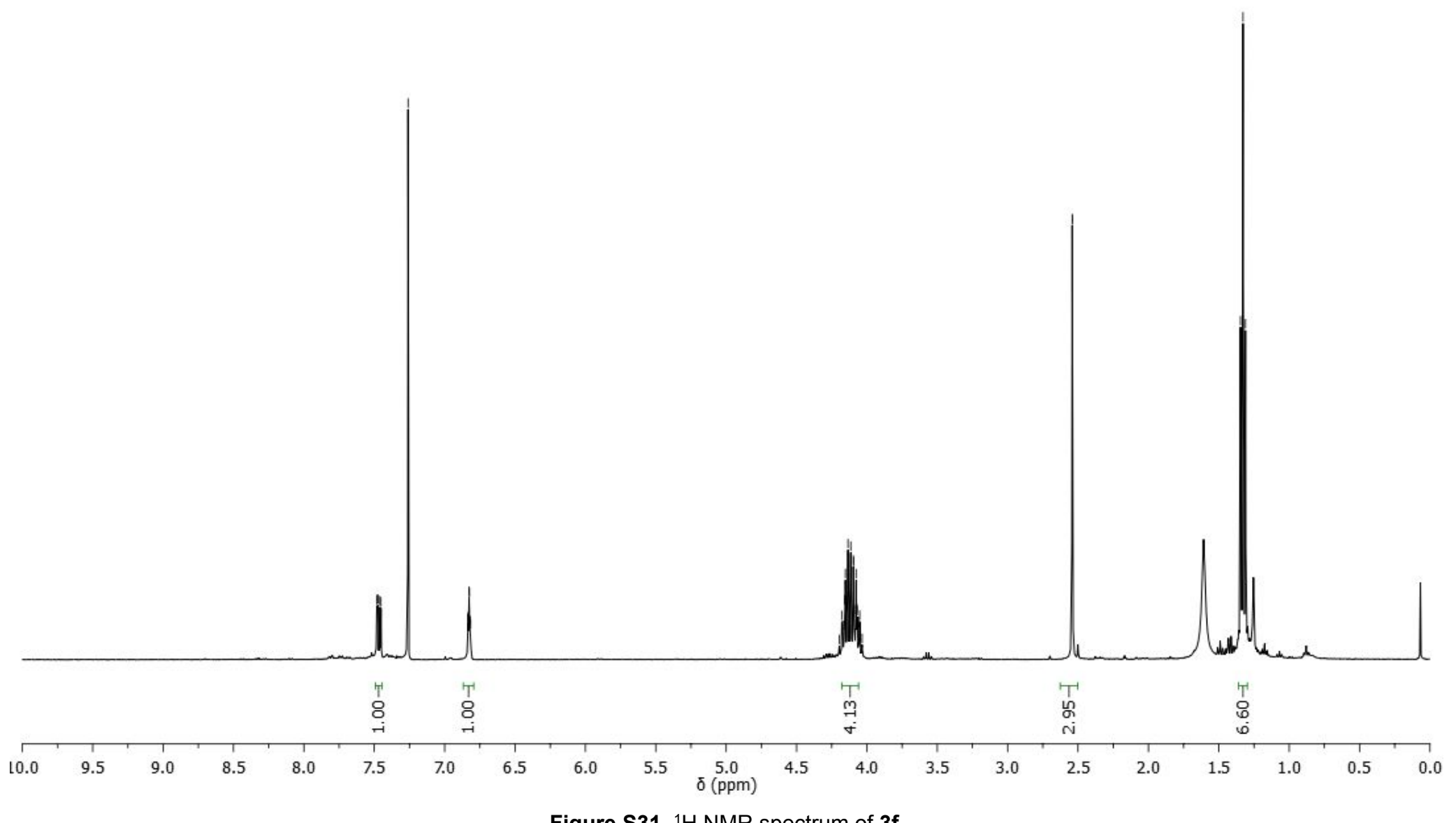

Figure S31. ${ }^{1} \mathrm{H}$ NMR spectrum of $3 \mathbf{f}$. 
Thiophene phosphonates

${ }^{13} \mathrm{C} 101 \mathrm{MHz}, \mathrm{CDCl}_{3}$

$3 f$

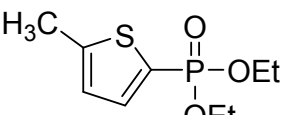

OEt

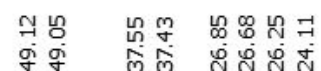

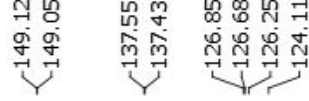

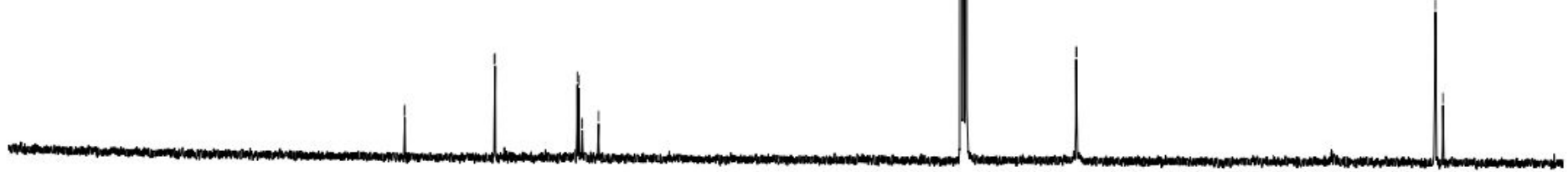

$\begin{array}{lllllll}200 & 190 & 180 & 170 & 160 & 150 & 140\end{array}$

$\begin{array}{ll}110 & 100 \\ \delta(\mathrm{ppm})\end{array}$

Figure S32. ${ }^{13} \mathrm{C}$ NMR spectrum of $\mathbf{3 f}$. 
${ }^{31} \mathrm{P} 162 \mathrm{MHz}, \mathrm{CDCl}_{3}$

$$
\begin{aligned}
& 3 f \stackrel{\mathrm{H}_{3} \mathrm{C}}{\mathrm{O}} \\
& \text { 궁 }
\end{aligned}
$$

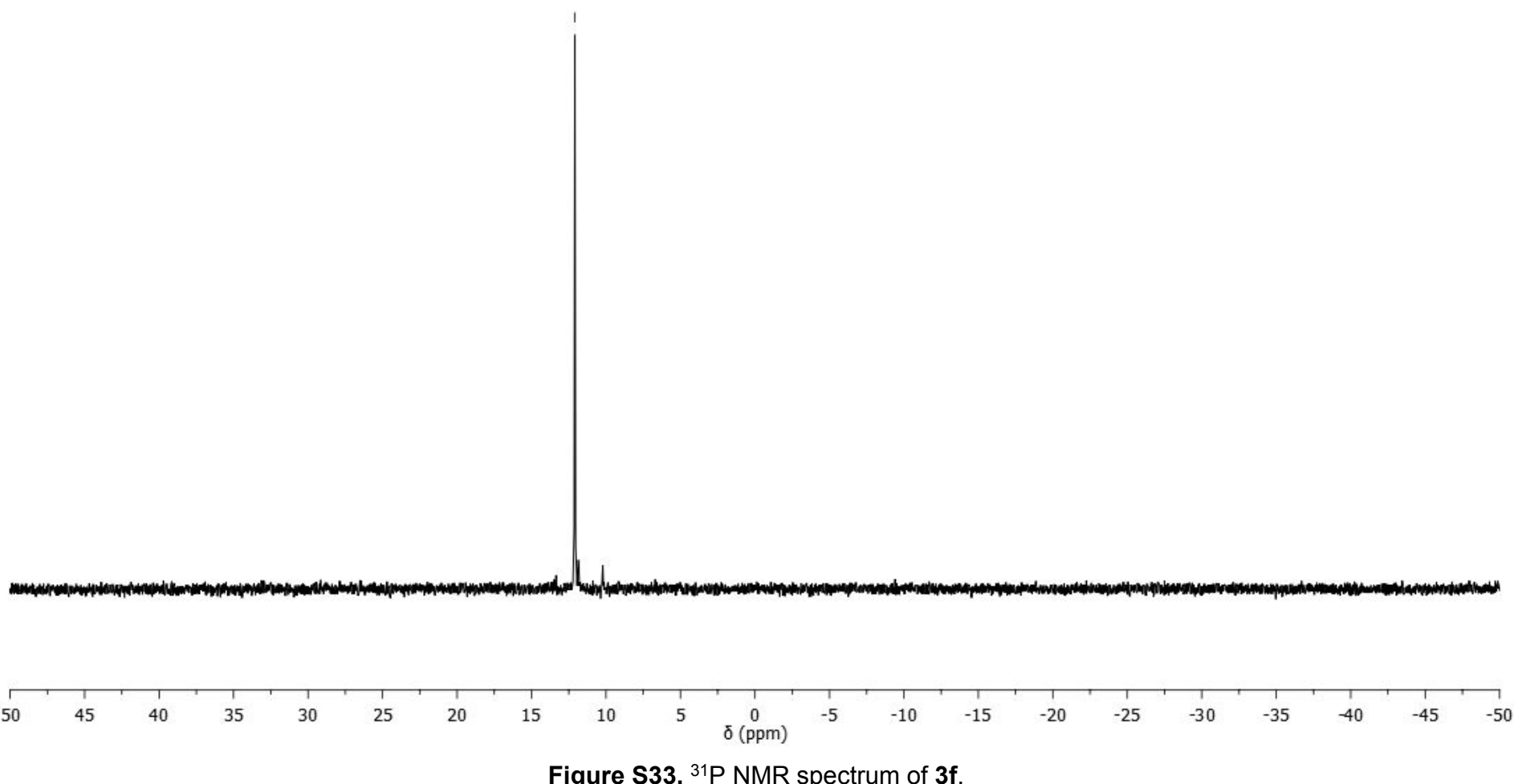

Figure S33. ${ }^{31} \mathrm{P}$ NMR spectrum of $\mathbf{3 f}$ 
Thiophene phosphonates

${ }^{1} \mathrm{H} 400 \mathrm{MHz}, \mathrm{CDCl}_{3}$

$3 g$ EtO, OEt

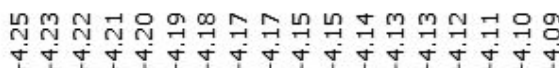

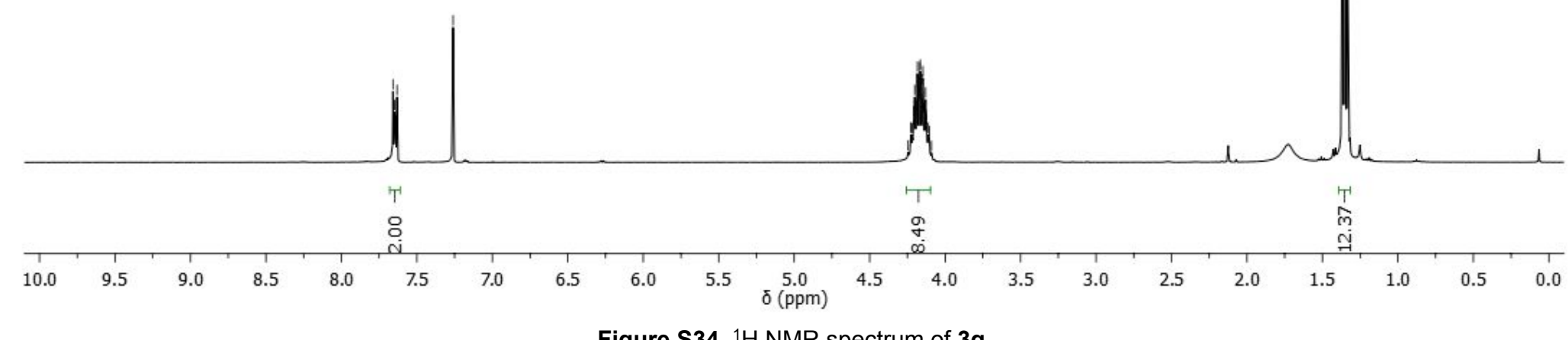

Figure S34. ${ }^{1} \mathrm{H}$ NMR spectrum of $\mathbf{3 g}$. 
Thiophene phosphonates

${ }^{13} \mathrm{C} 101 \mathrm{MHz}, \mathrm{CDCl}_{3}$

EtO、, OEt

$$
\text { O II }
$$

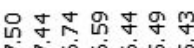

ming

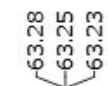

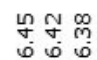

每

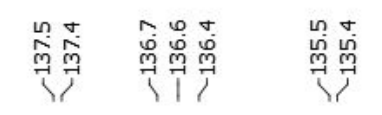

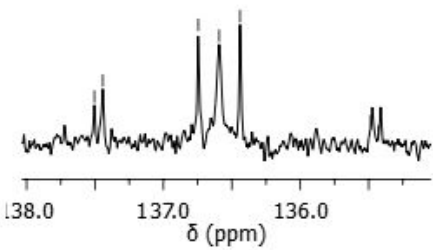

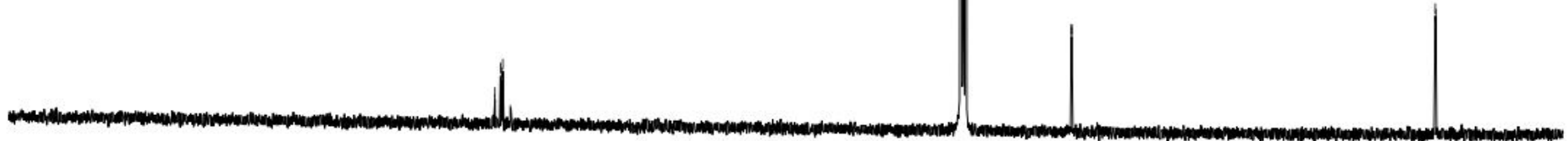

$\begin{array}{llllllll}200 & 190 & 180 & 170 & 160 & 150 & 140 & 130\end{array}$

Figure S35. ${ }^{13} \mathrm{C}$ NMR spectrum of $\mathbf{3 g}$. 
${ }^{31} \mathrm{P} 162 \mathrm{MHz}, \mathrm{CDCl}_{3}$

$$
\begin{aligned}
& 3 \text { EtO, OEt } \\
& \text { 出 O }
\end{aligned}
$$

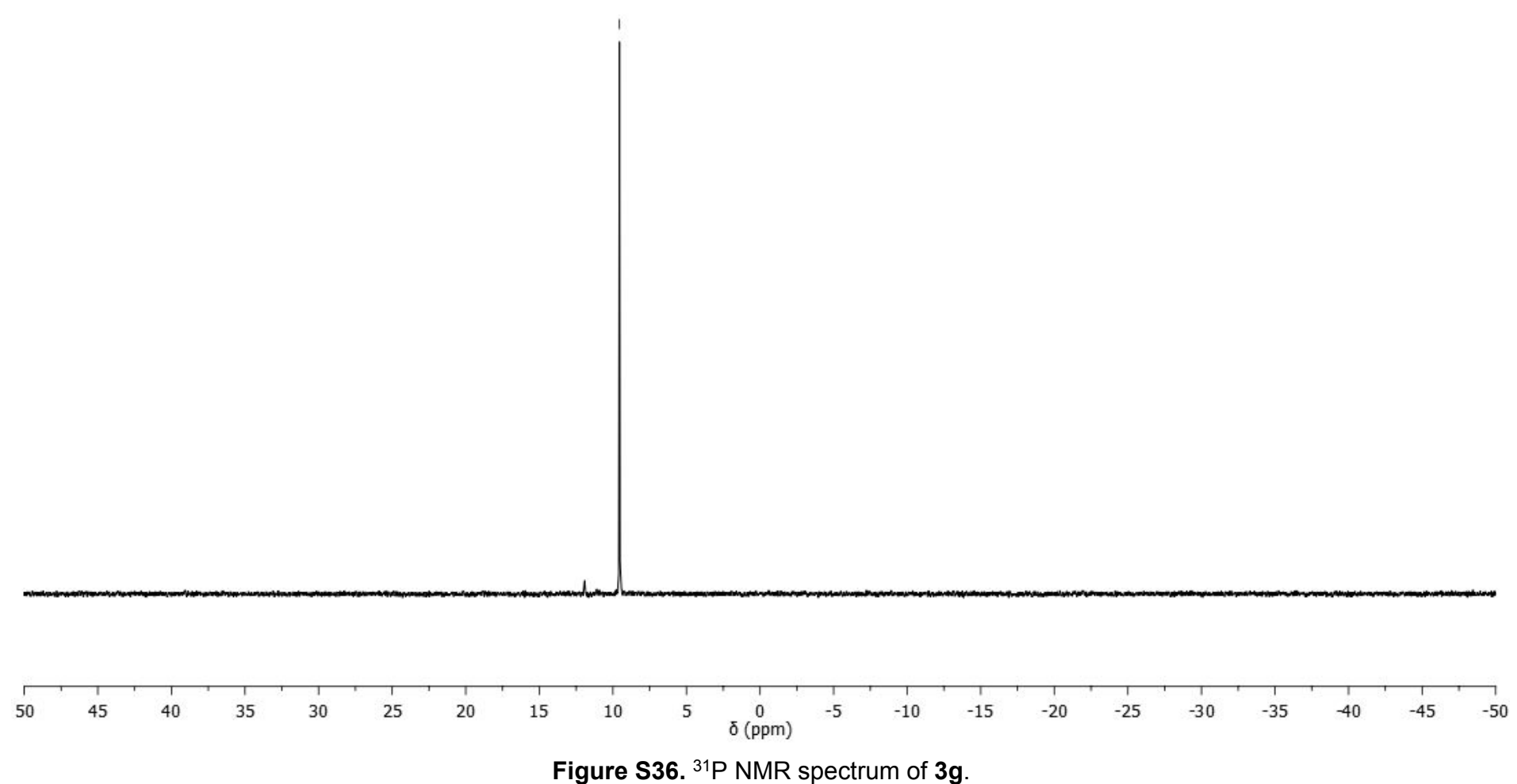


Thiophene phosphonates

${ }^{1} \mathrm{H} 400 \mathrm{MHz}, \mathrm{CDCl}_{3}$

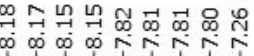

3h

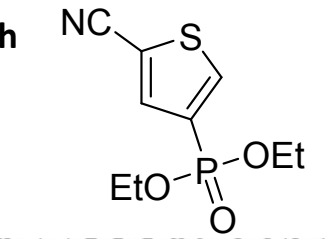

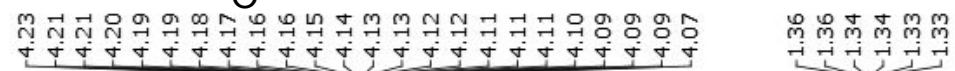

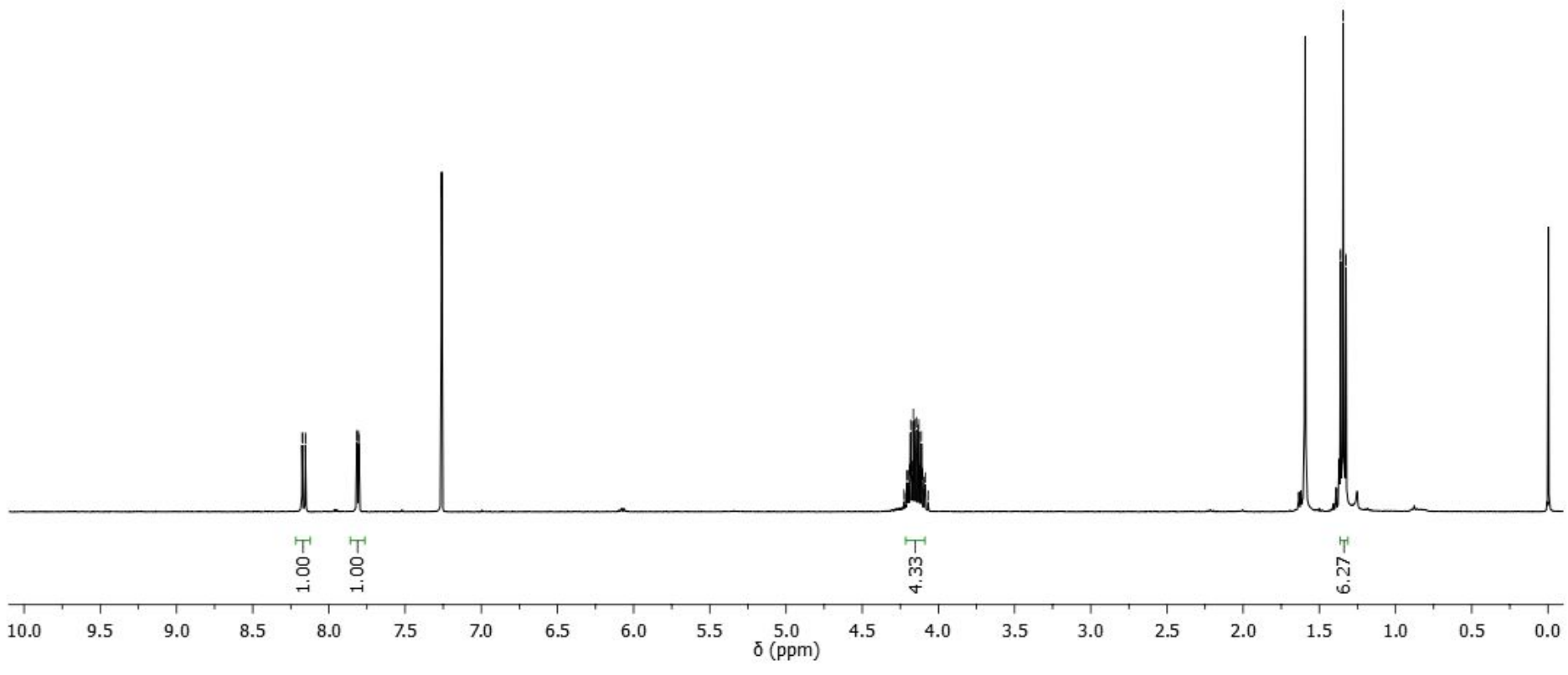

Figure S37. ${ }^{1} \mathrm{H}$ NMR spectrum of $3 \mathrm{~h}$. 
Thiophene phosphonates

${ }^{13} \mathrm{C} 101 \mathrm{MHz}, \mathrm{CDCl}_{3}$

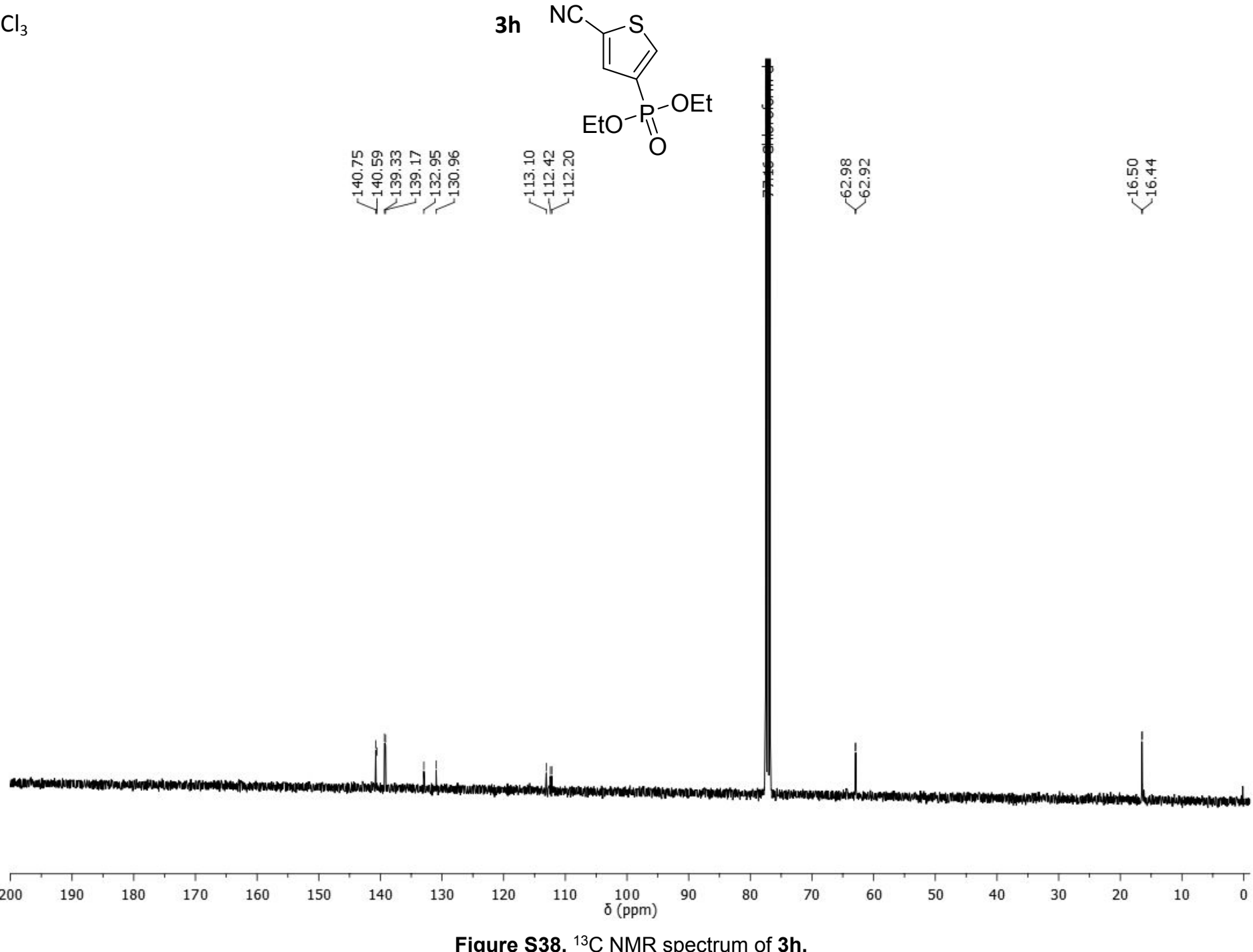

Figure S38. ${ }^{13} \mathrm{C}$ NMR spectrum of $\mathbf{3 h}$. 
${ }^{31} \mathrm{P} 162 \mathrm{MHz}, \mathrm{CDCl}_{3}$
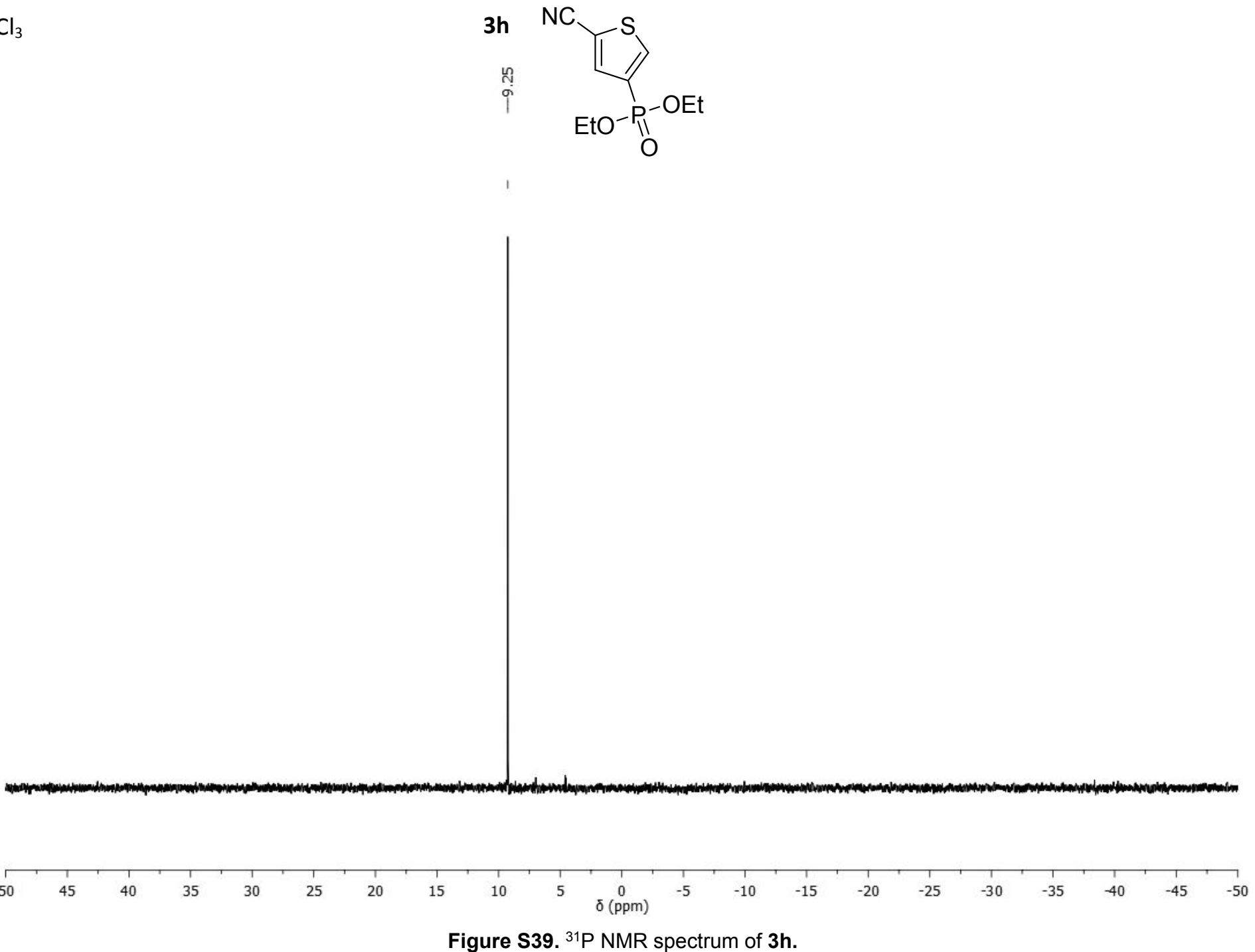

Figure S39. ${ }^{31} \mathrm{P}$ NMR spectrum of $3 \mathrm{~h}$ 
Thiophene phosphonates

${ }^{1} \mathrm{H} 400 \mathrm{MHz}, \mathrm{CDCl}_{3}$

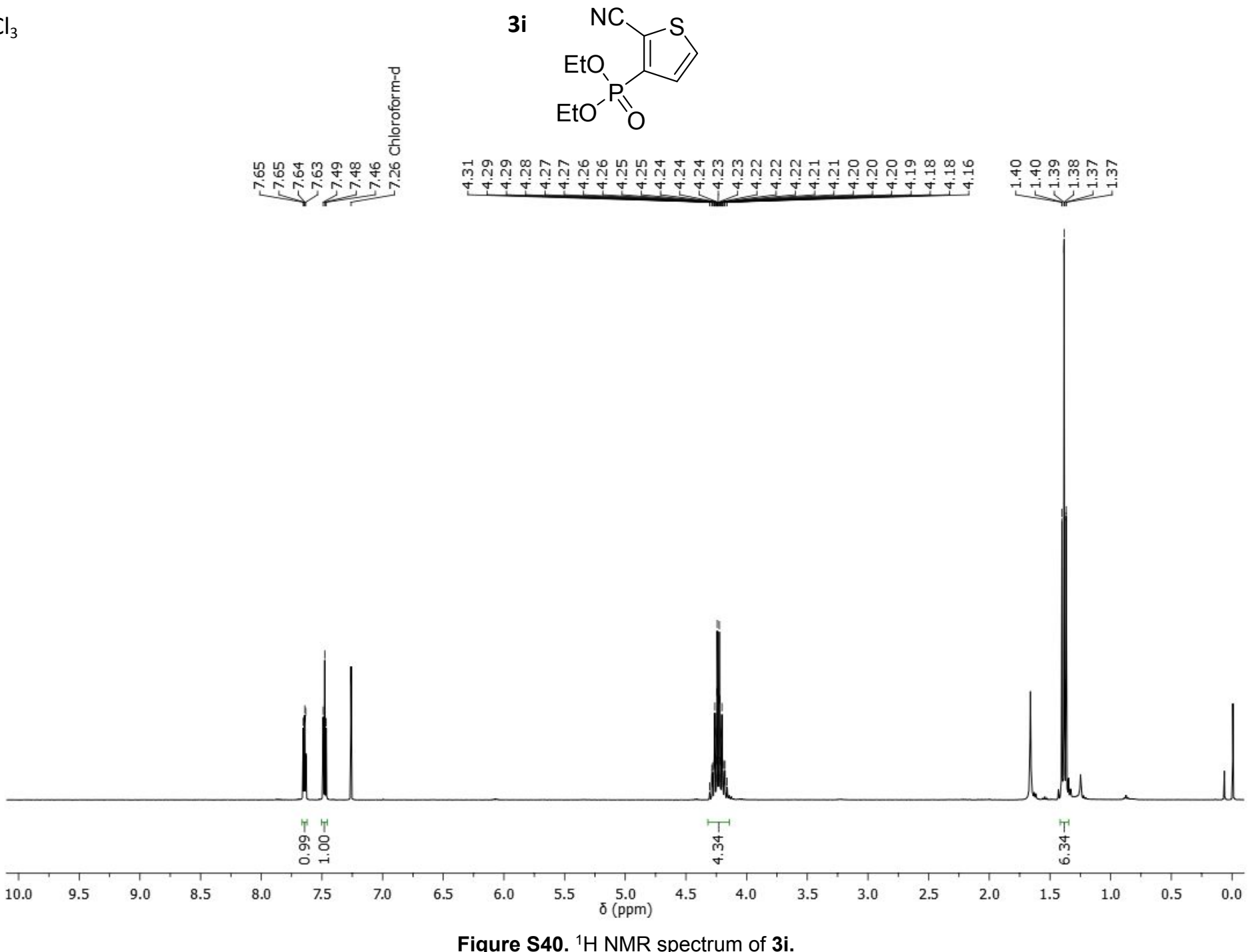


Thiophene phosphonates

${ }^{13} \mathrm{C} 101 \mathrm{MHz}, \mathrm{CDCl}_{3}$

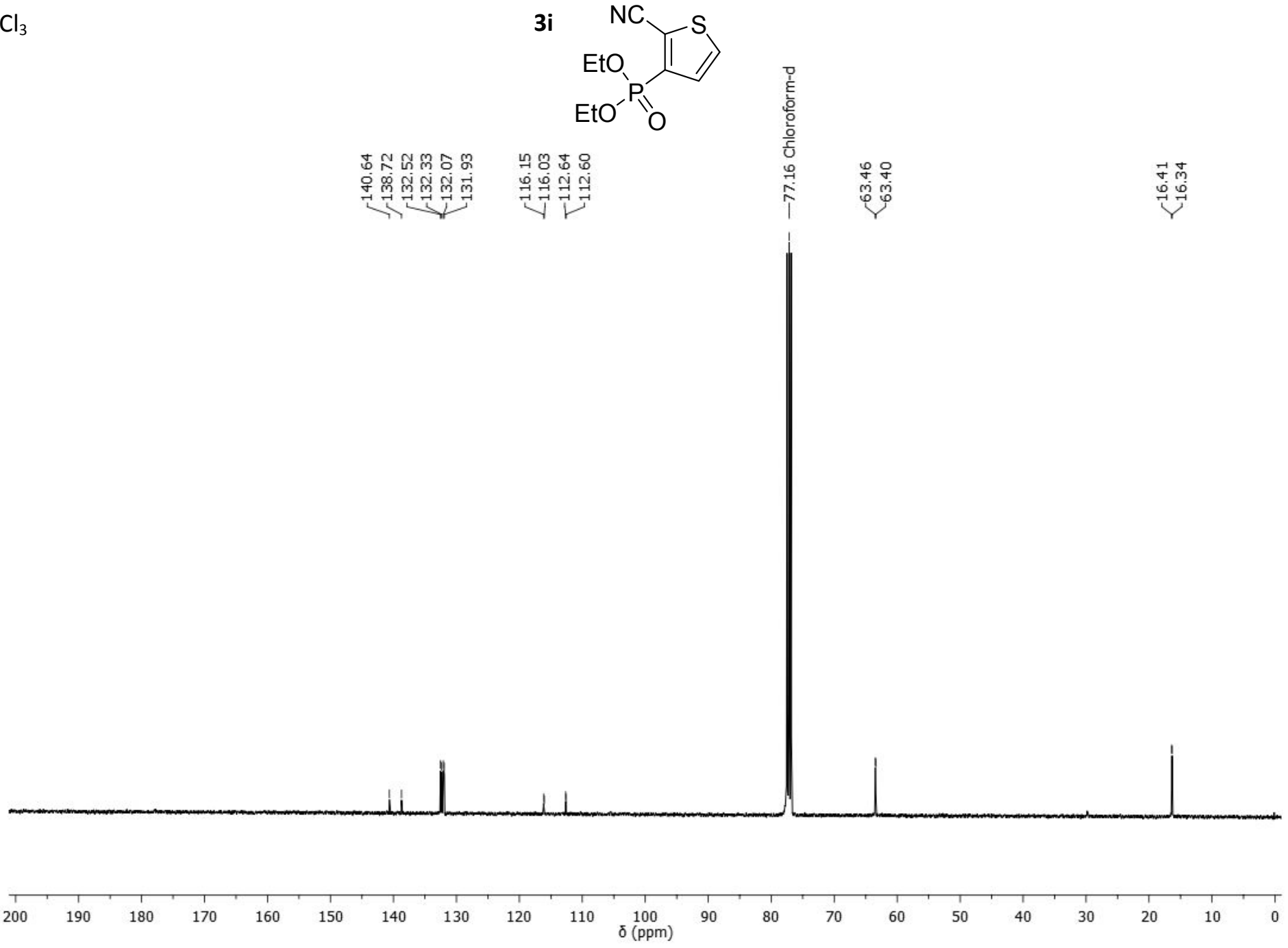

Figure $\mathbf{S} 41 .{ }^{13} \mathrm{C}$ NMR spectrum of $\mathbf{3 i}$. 
${ }^{31} \mathrm{P} 162 \mathrm{MHz}, \mathrm{CDCl}_{3}$
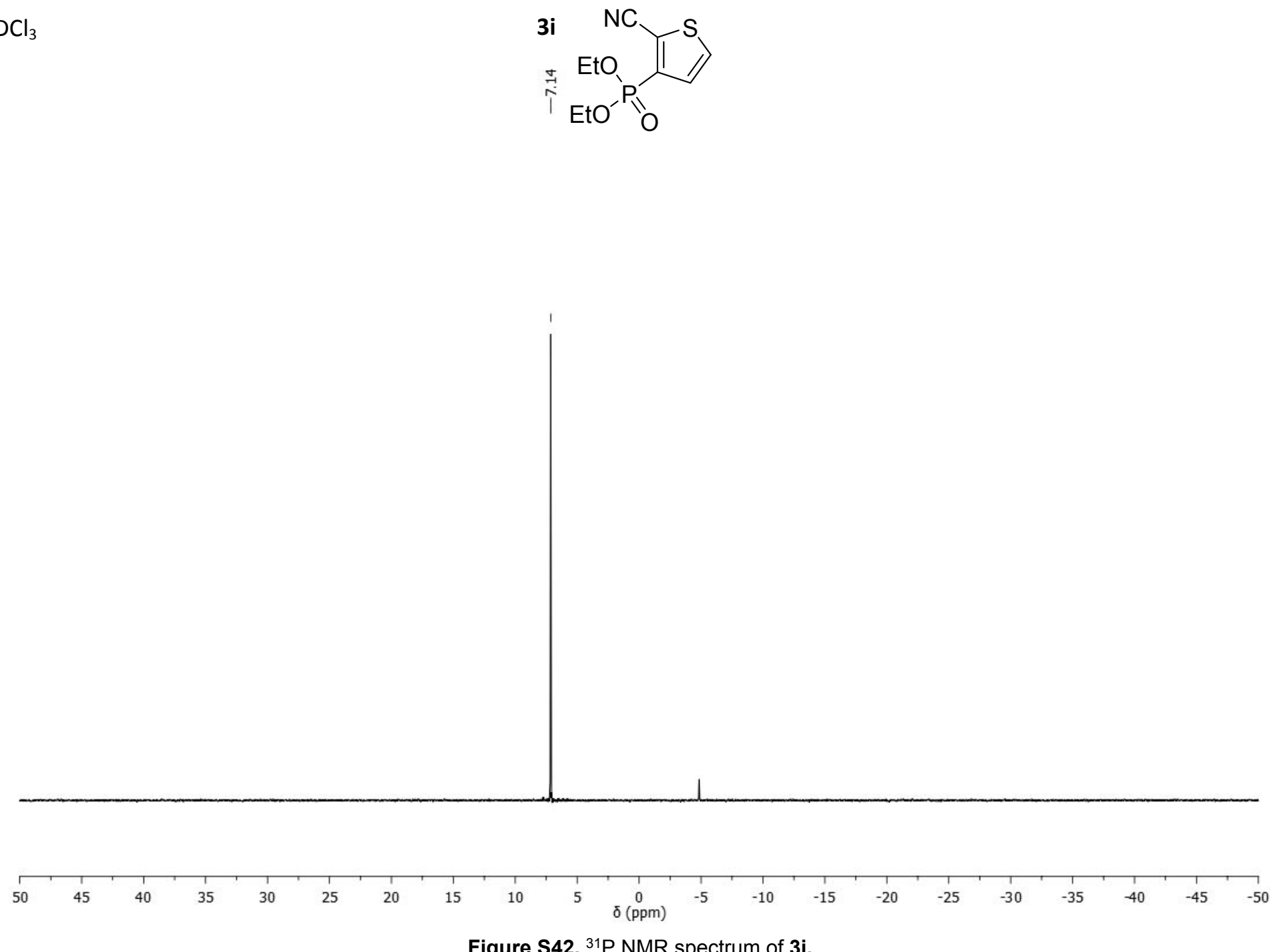

Figure S42. ${ }^{31} \mathrm{P}$ NMR spectrum of $\mathbf{3 i}$. 
Thiophene phosphonates

${ }^{1} \mathrm{H} 400 \mathrm{MHz}, \mathrm{CDCl}_{3}$

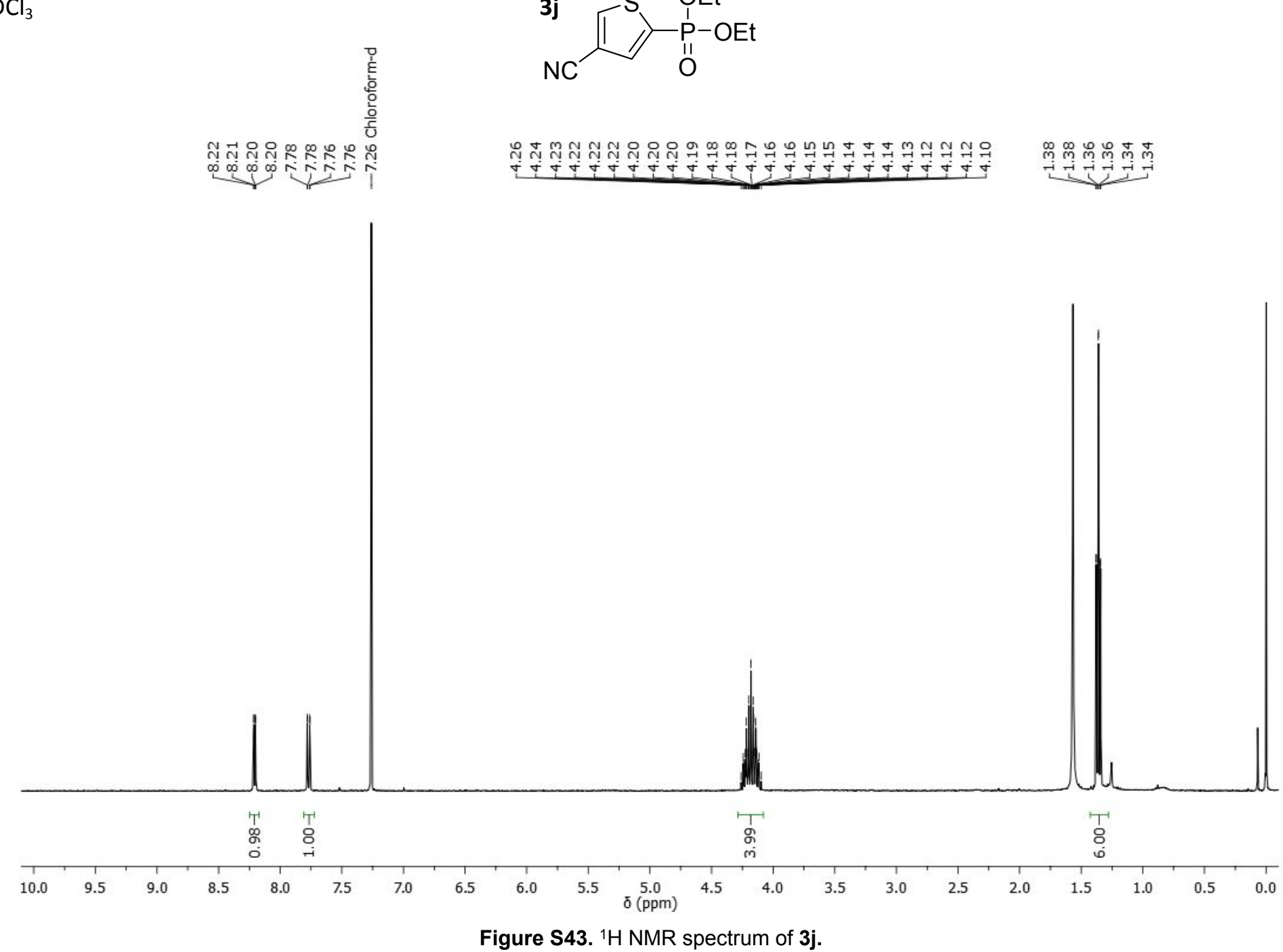


Thiophene phosphonates

${ }^{13} \mathrm{C} 101 \mathrm{MHz}, \mathrm{CDCl}_{3}$

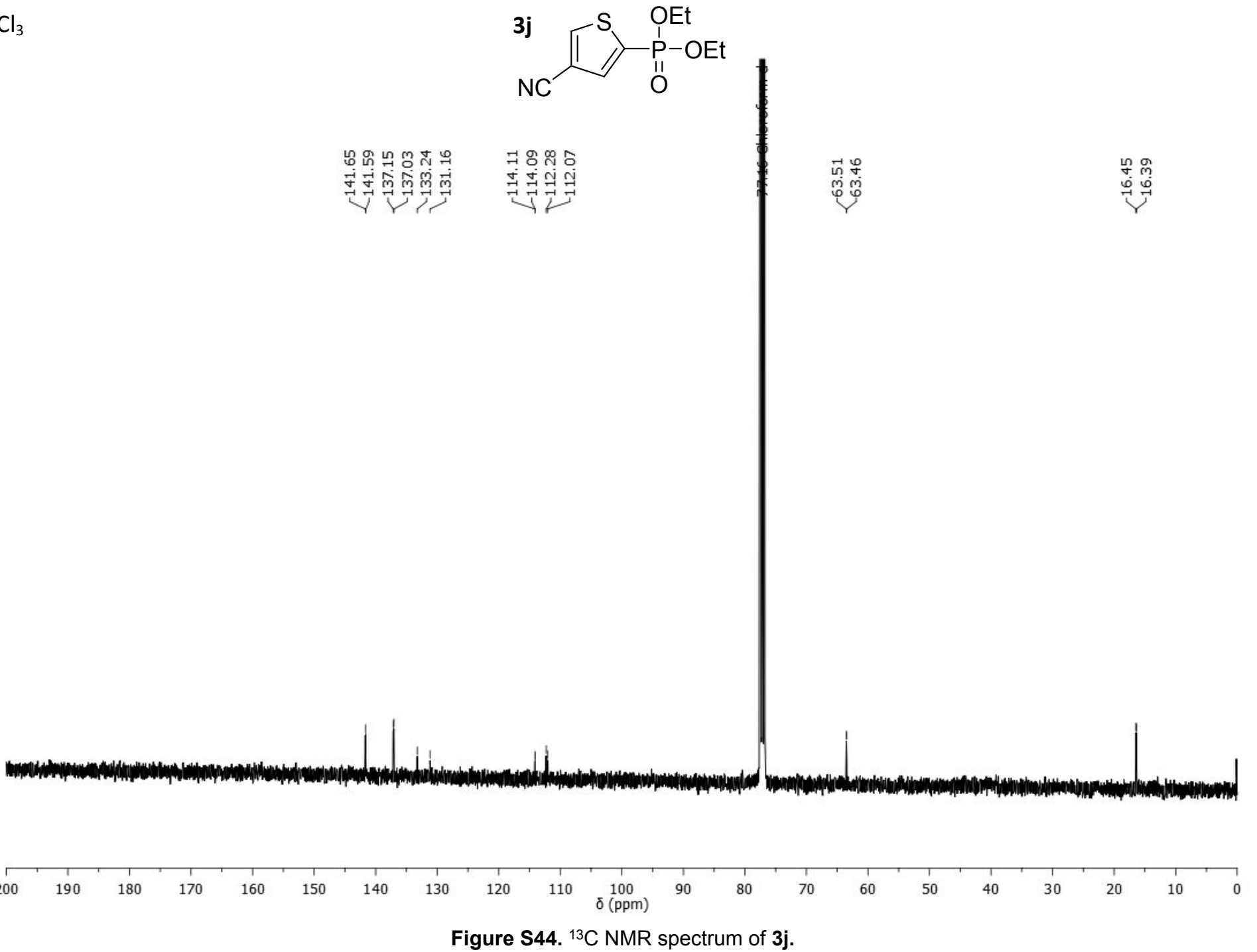

Figure $\mathbf{S 4 4 .}{ }^{13} \mathrm{C}$ NMR spectrum of $\mathbf{3 j}$. 
${ }^{31} \mathrm{P} 162 \mathrm{MHz}, \mathrm{CDCl}_{3}$

3j

$\mathrm{NC}_{\substack{\mathrm{O} \\ \mathrm{P}}}^{\mathrm{OEt}}$

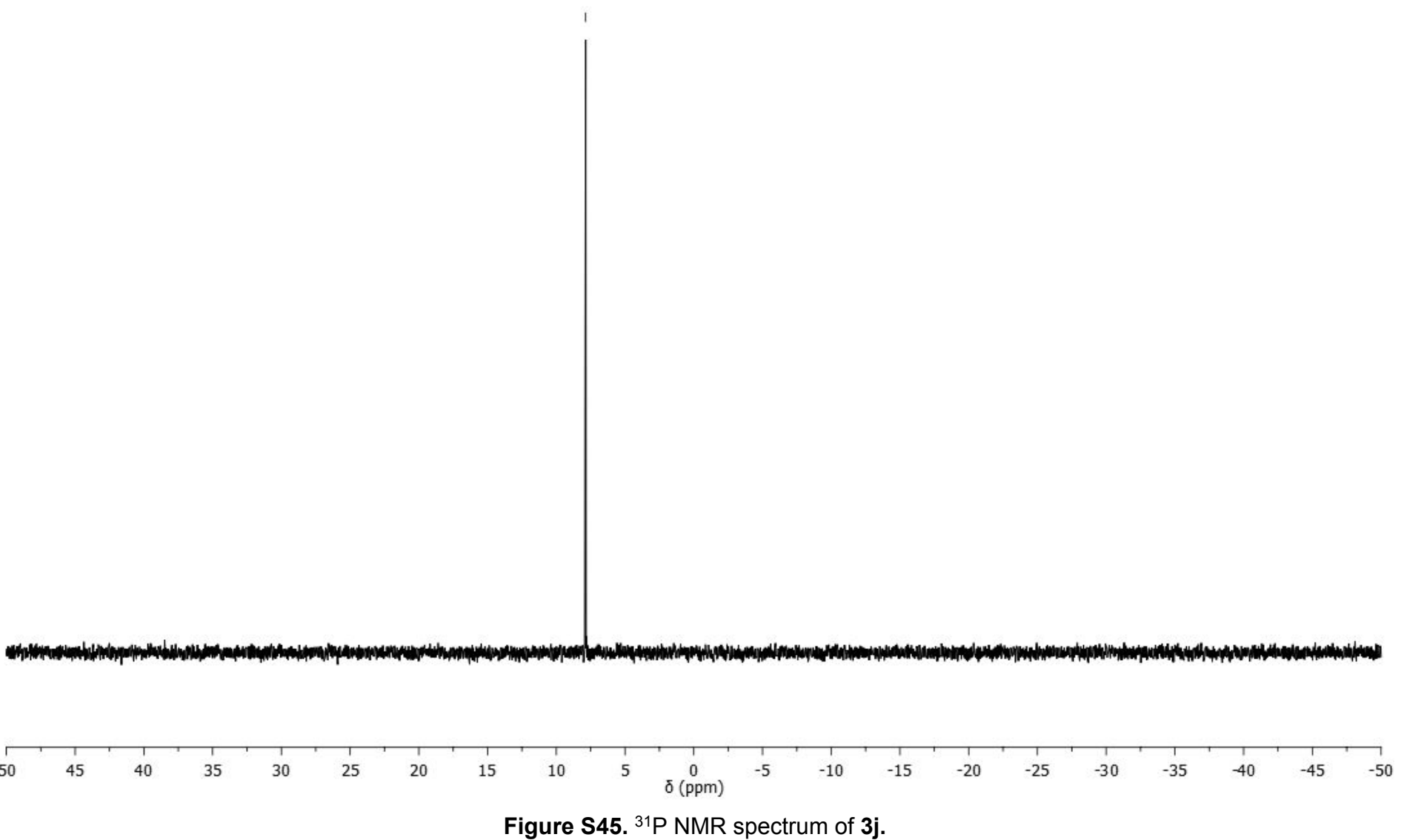

Figure S45. ${ }^{31} \mathrm{P}$ NMR spectrum of 3j. 
Thiophene phosphonates

${ }^{1} \mathrm{H} 400 \mathrm{MHz}, \mathrm{CDCl}_{3}$

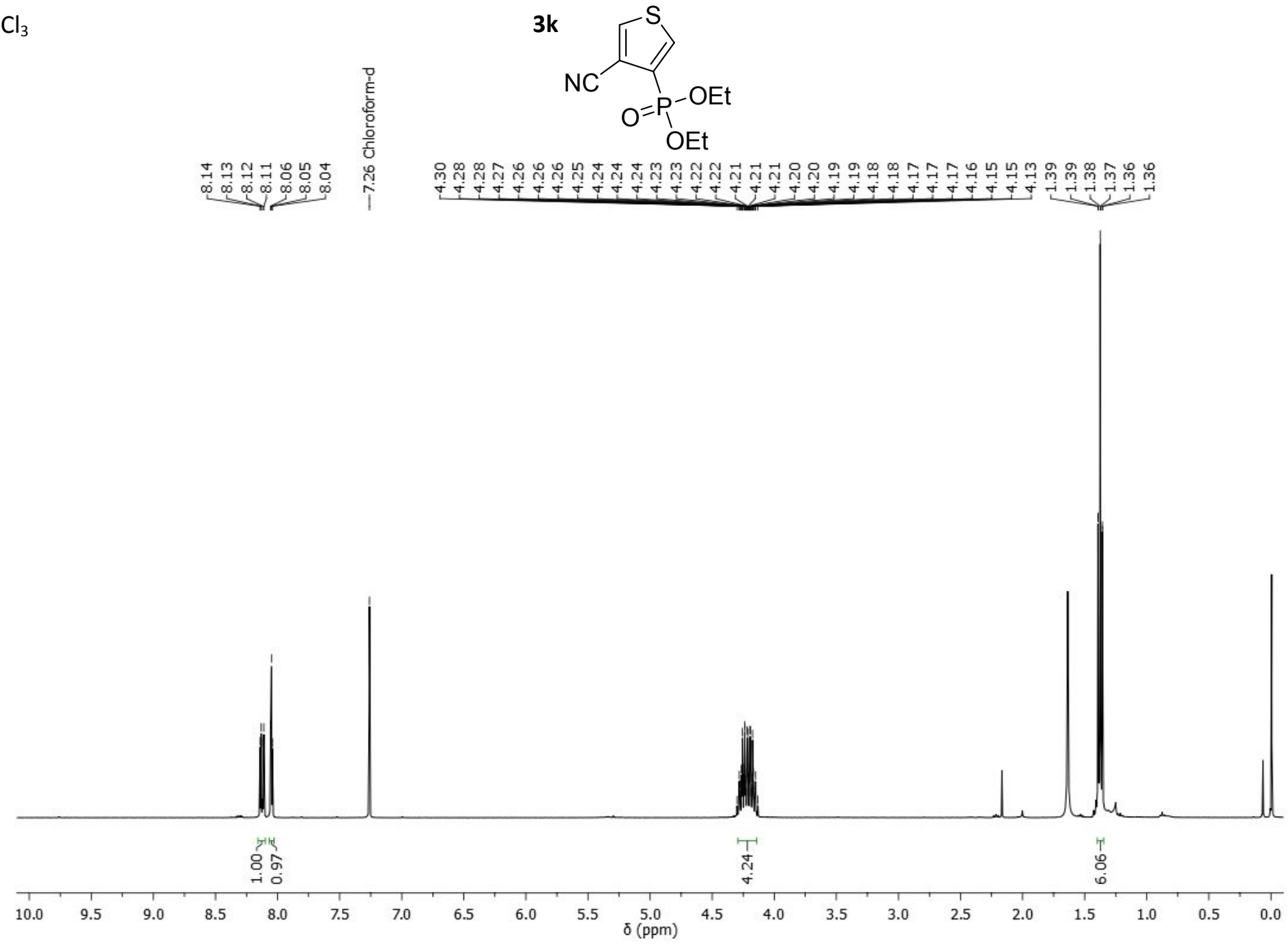

Figure S46. ${ }^{1} \mathrm{H}$ NMR spectrum of $\mathbf{3 k}$. 
Thiophene phosphonates

${ }^{13} \mathrm{C} 101 \mathrm{MHz}, \mathrm{CDCl}_{3}$

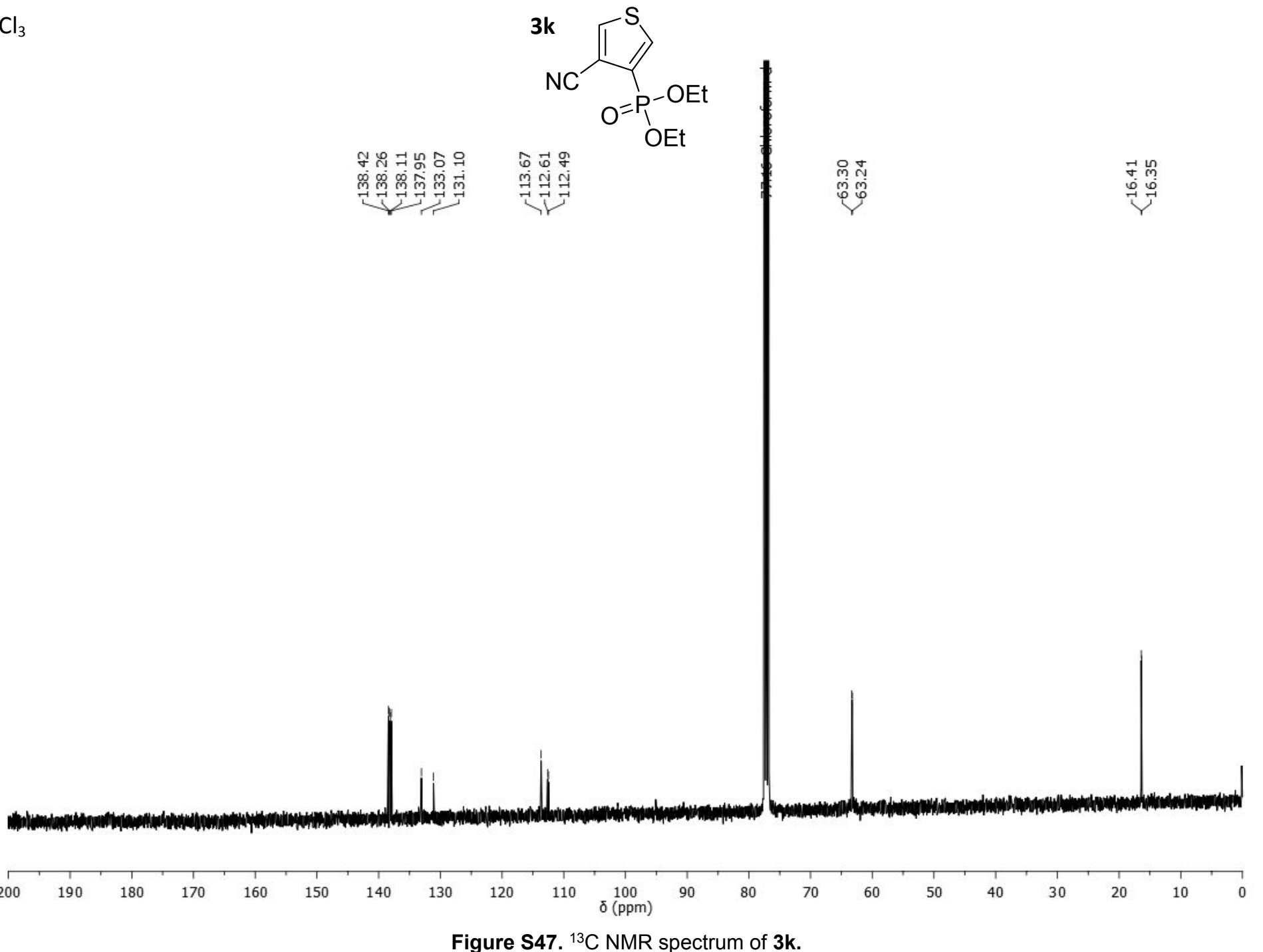


${ }^{31} \mathrm{P} 162 \mathrm{MHz}, \mathrm{CDCl}_{3}$
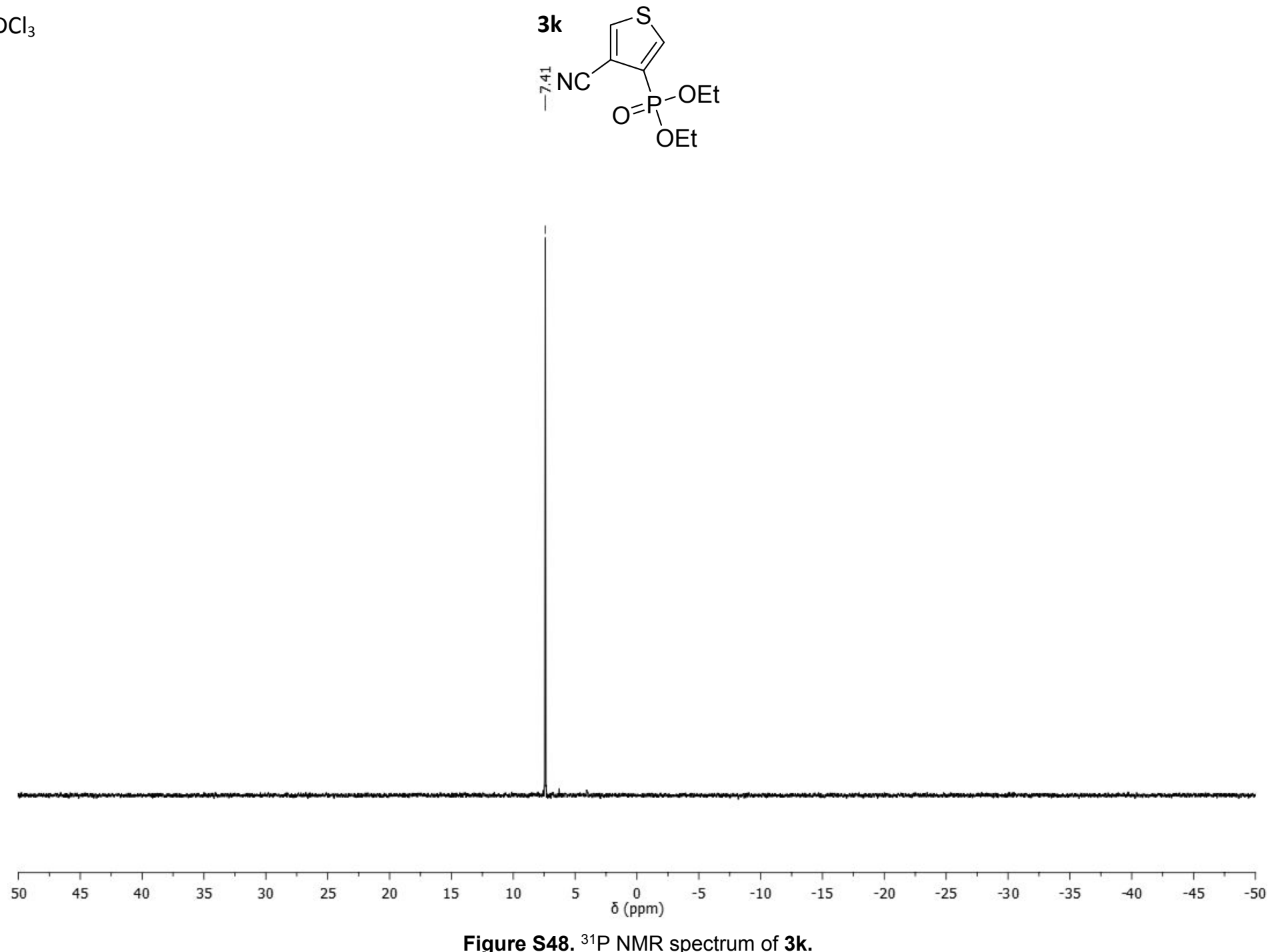
Thiophene phosphonates

${ }^{1} \mathrm{H} 400 \mathrm{MHz}, \mathrm{CDCl}_{3}$

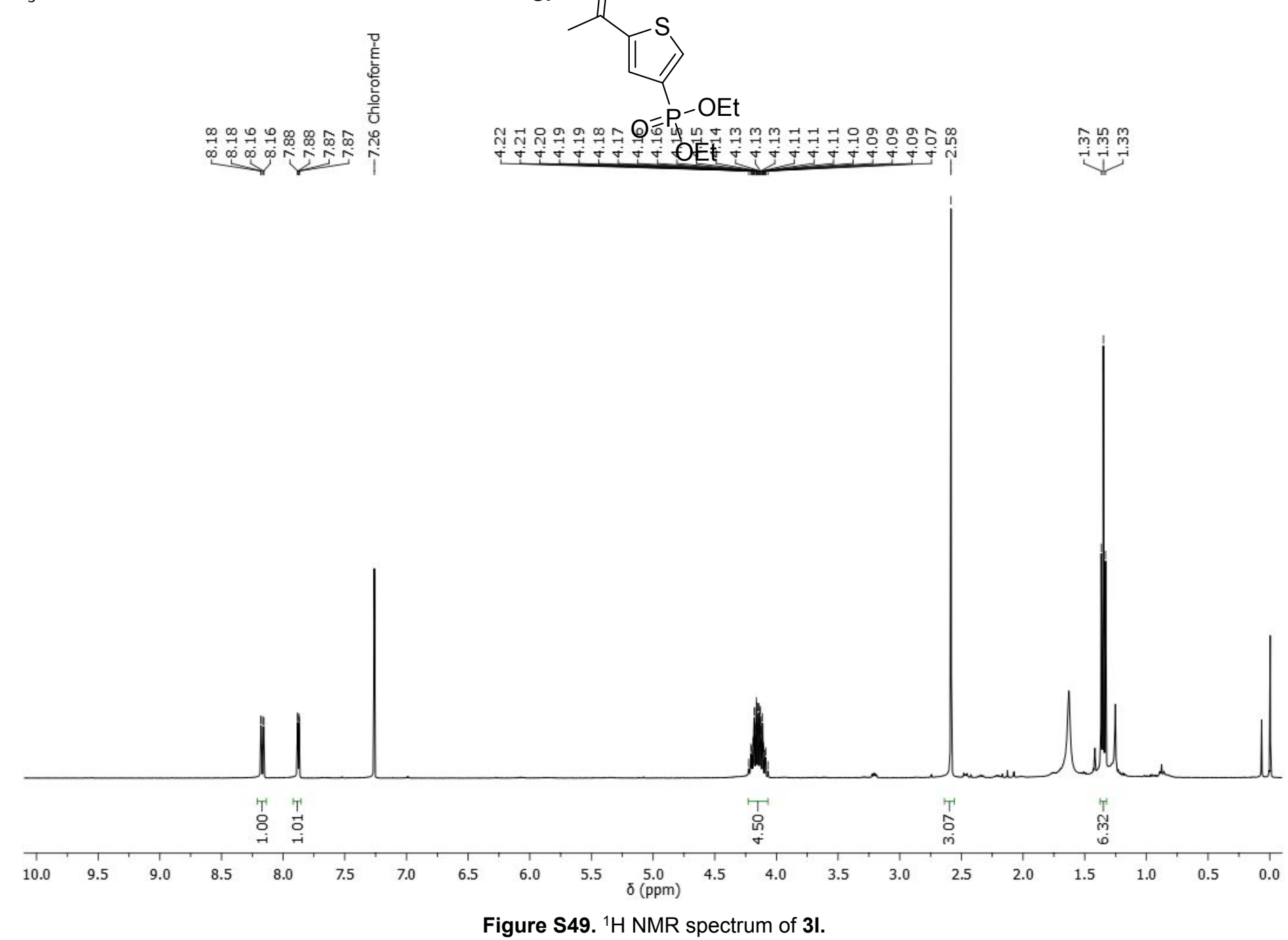

Figure S49. ${ }^{1} \mathrm{H}$ NMR spectrum of 31. 
Thiophene phosphonates

${ }^{13} \mathrm{C} 101 \mathrm{MHz}, \mathrm{CDCl}_{3}$

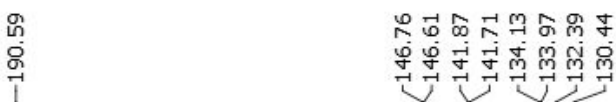

31

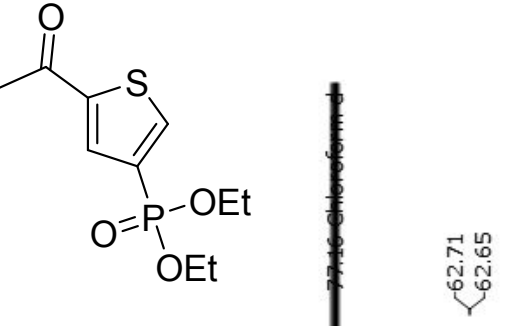

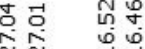

章

Figure S50. ${ }^{13} \mathrm{C}$ NMR spectrum of 3 I. 
${ }^{31} \mathrm{P} 162 \mathrm{MHz} \mathrm{CDCl}_{3}$
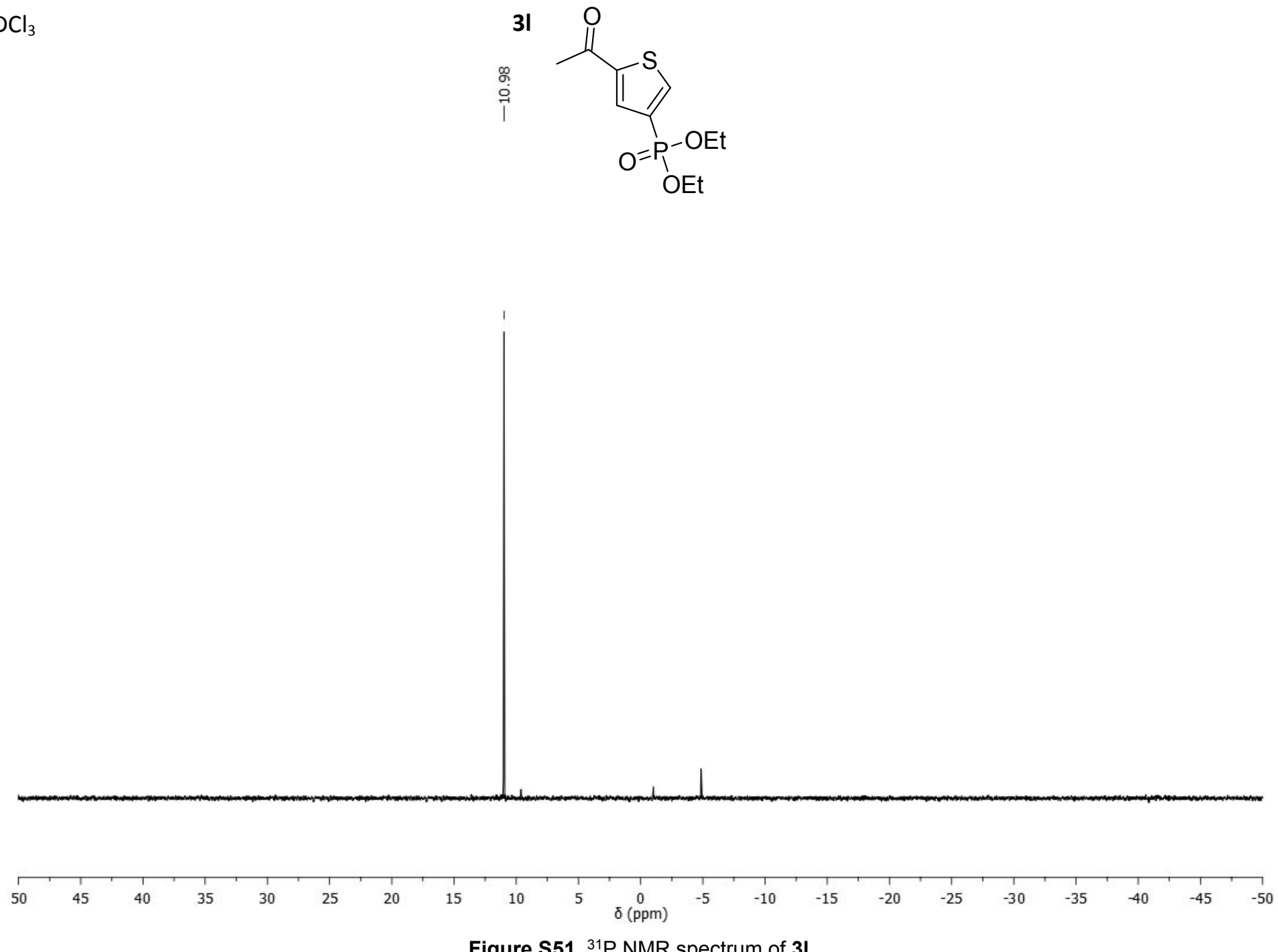

Figure S51. ${ }^{31} \mathrm{P}$ NMR spectrum of 31. 
Thiophene phosphonates

${ }^{1} \mathrm{H} 400 \mathrm{MHz}, \mathrm{CDCl}_{3}$

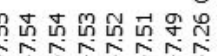

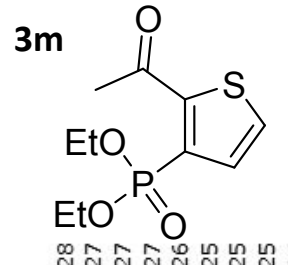

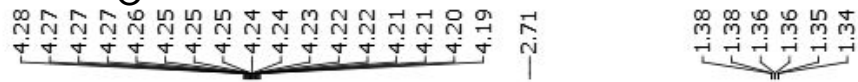

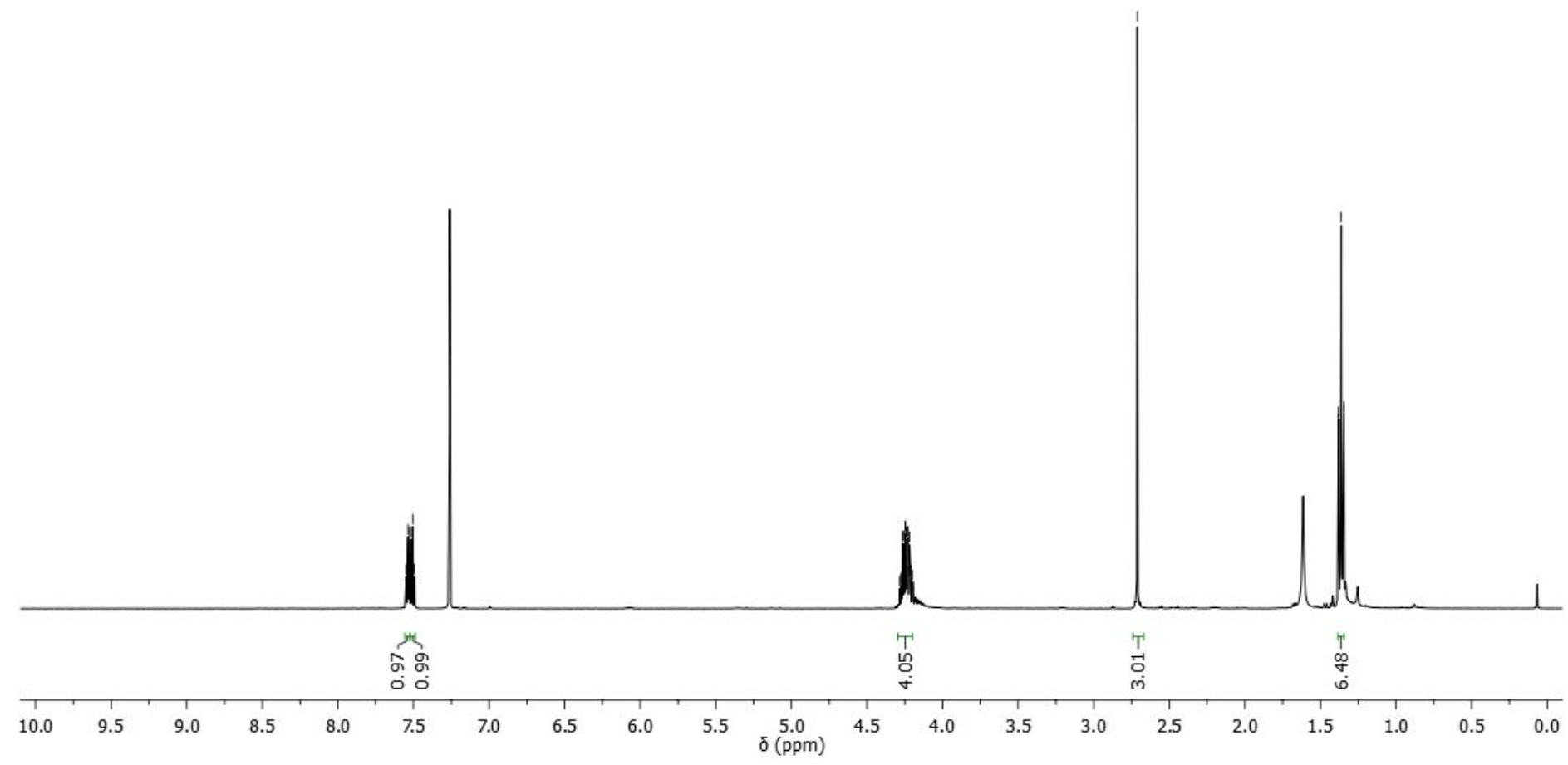

Figure S52. ${ }^{1} \mathrm{H}$ NMR spectrum of $3 \mathrm{~m}$. 
Thiophene phosphonates

${ }^{13} \mathrm{C} 101 \mathrm{MHz}, \mathrm{CDCl}_{3}$

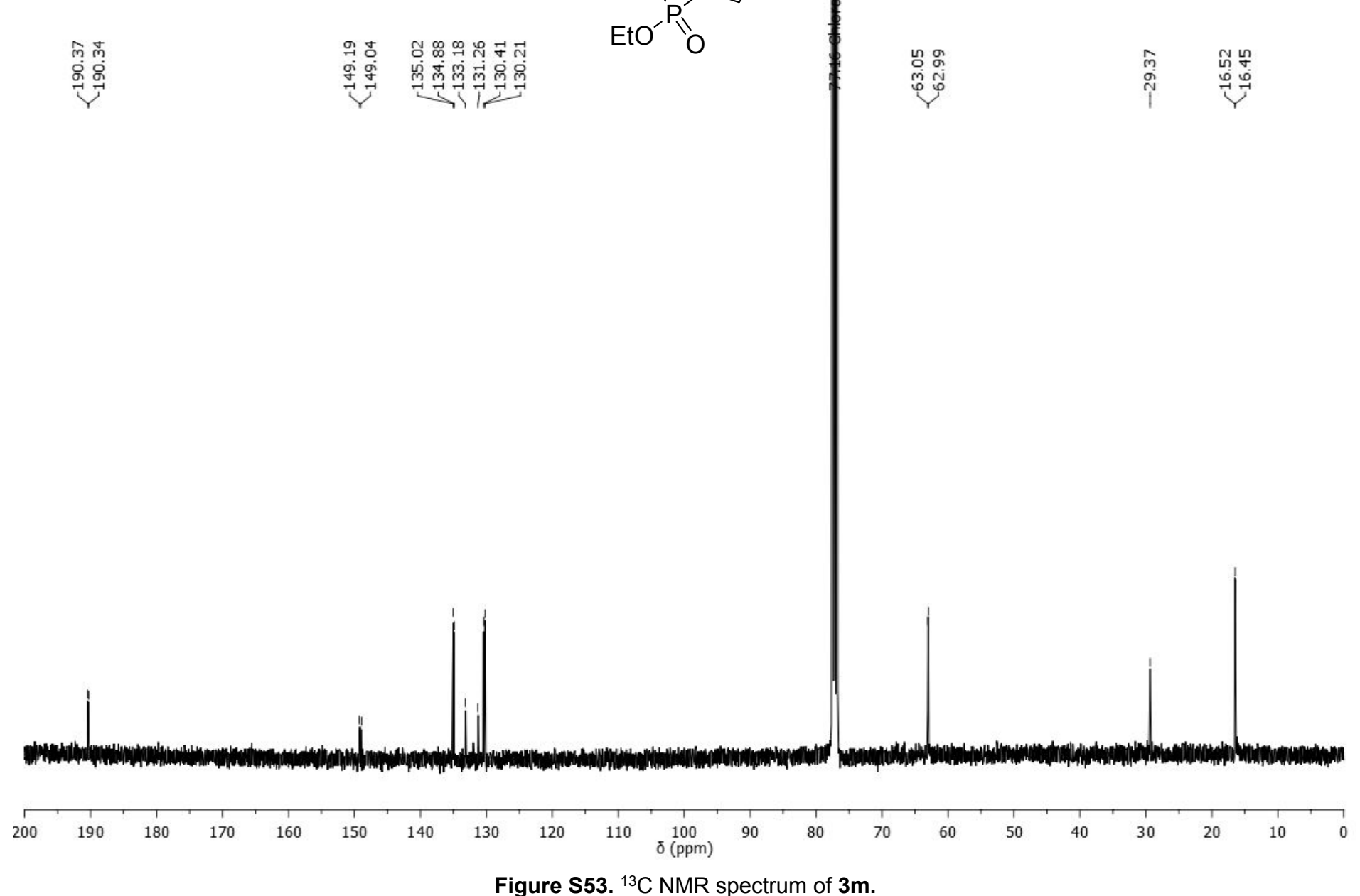


${ }^{31} \mathrm{P} 162 \mathrm{MHz}, \mathrm{CDCl}_{3}$
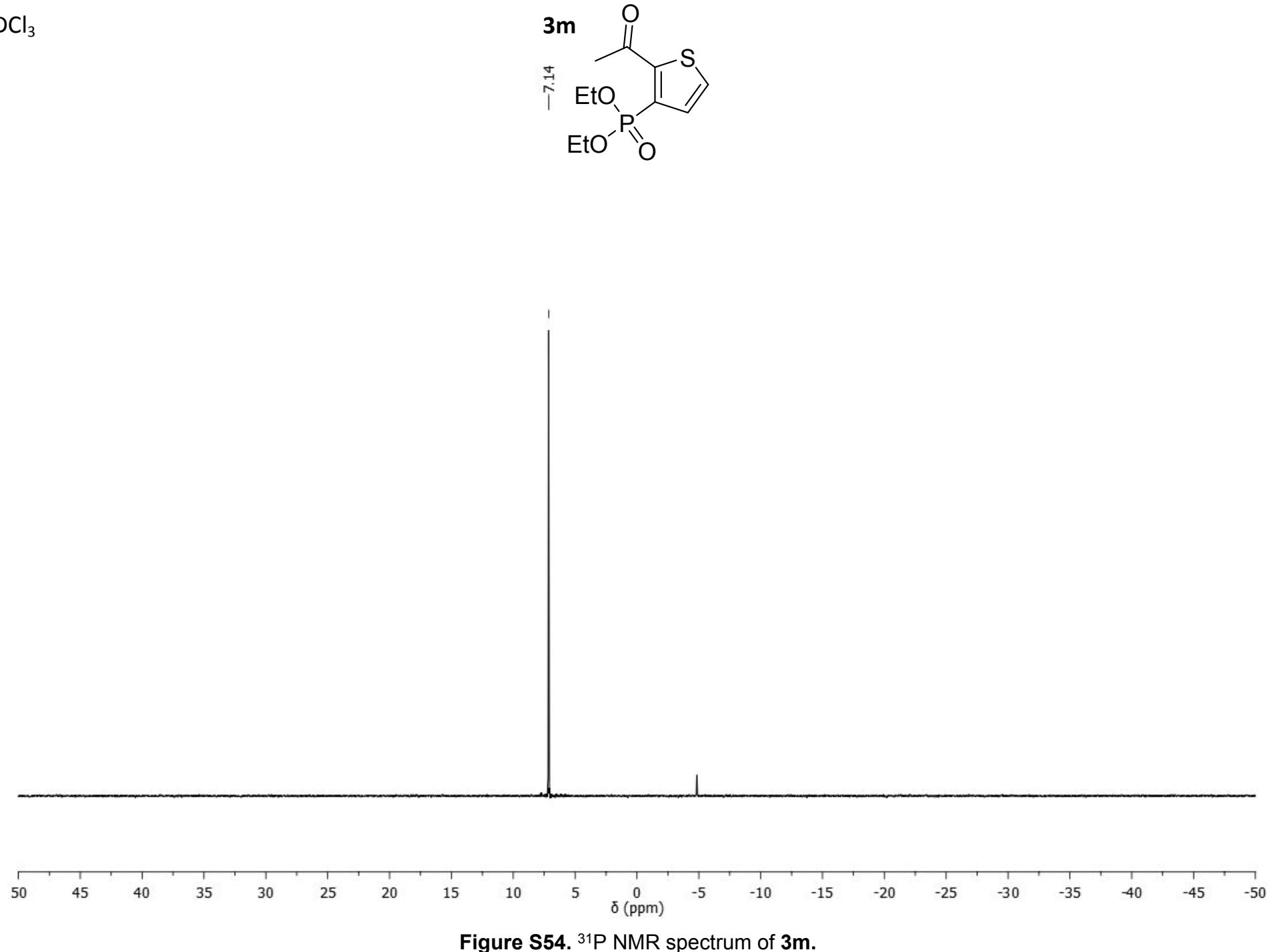
Thiophene phosphonates

${ }^{1} \mathrm{H} 400 \mathrm{MHz}, \mathrm{CDCl}_{3}$

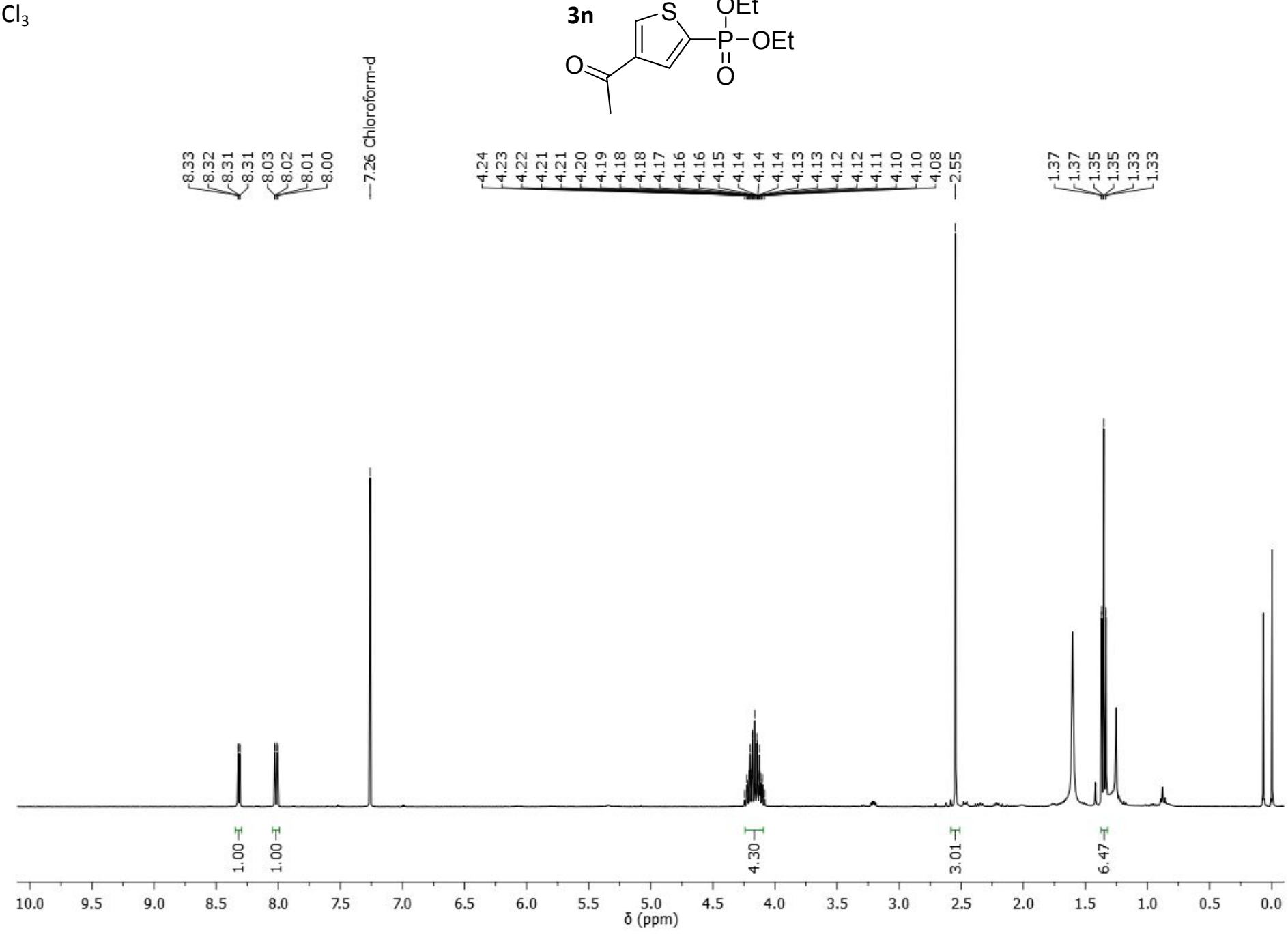

Figure S55. ${ }^{1} \mathrm{H}$ NMR spectrum of $\mathbf{3 n}$. 
Thiophene phosphonates

${ }^{13} \mathrm{C} 101 \mathrm{MHz}, \mathrm{CDCl}_{3}$
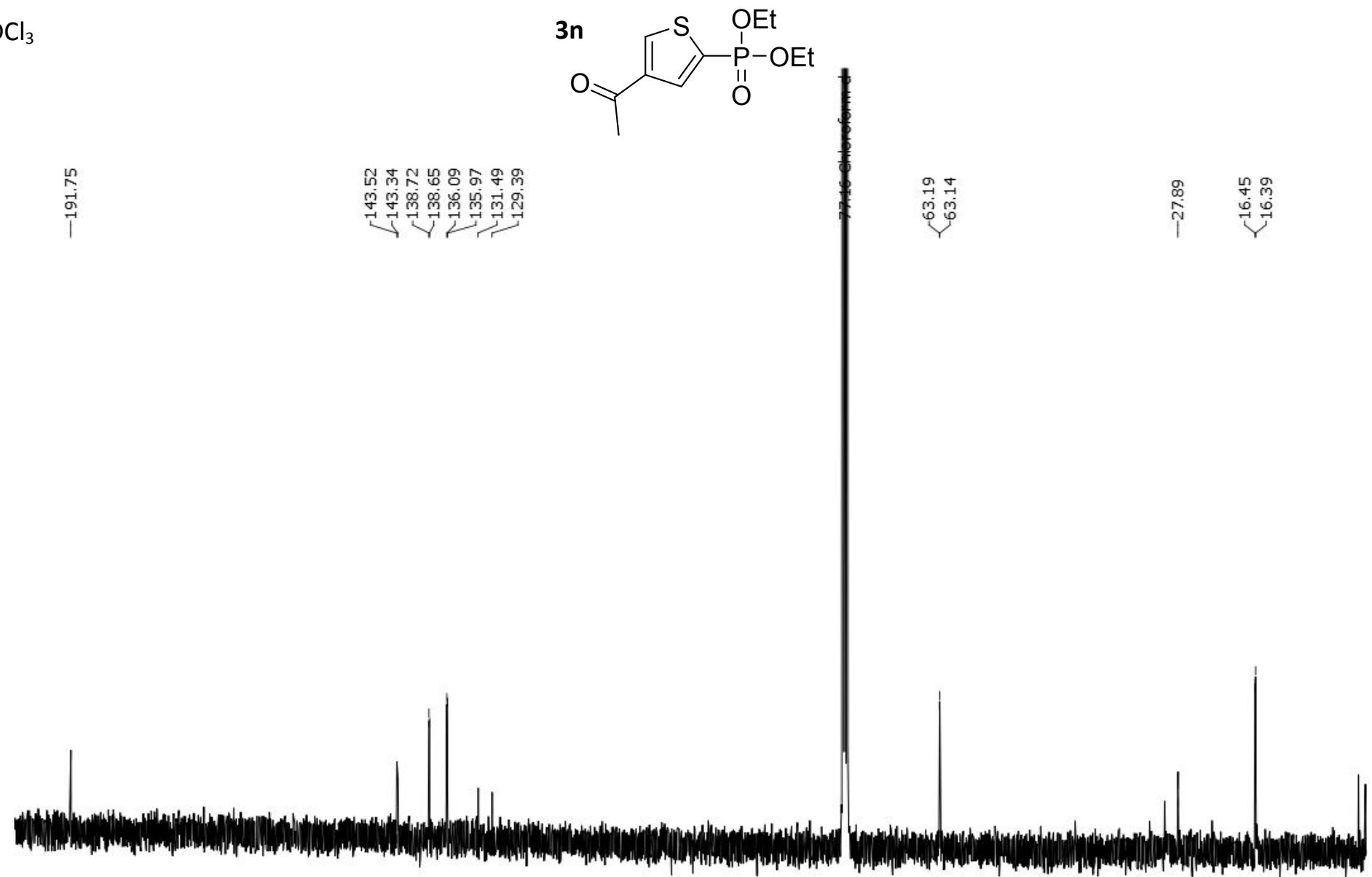

$200 \quad 190 \quad 180$

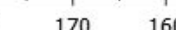

130

$\begin{array}{lll}120 & 110 & 100 \\ \delta(\mathrm{ppm})\end{array}$

Figure S56. ${ }^{13} \mathrm{C}$ NMR spectrum of $3 n$. 
Thiophene phosphonates

${ }^{31} \mathrm{P} 162 \mathrm{MHz}, \mathrm{CDCl}_{3}$

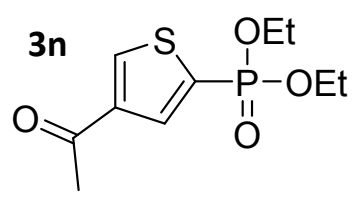


Thiophene phosphonates
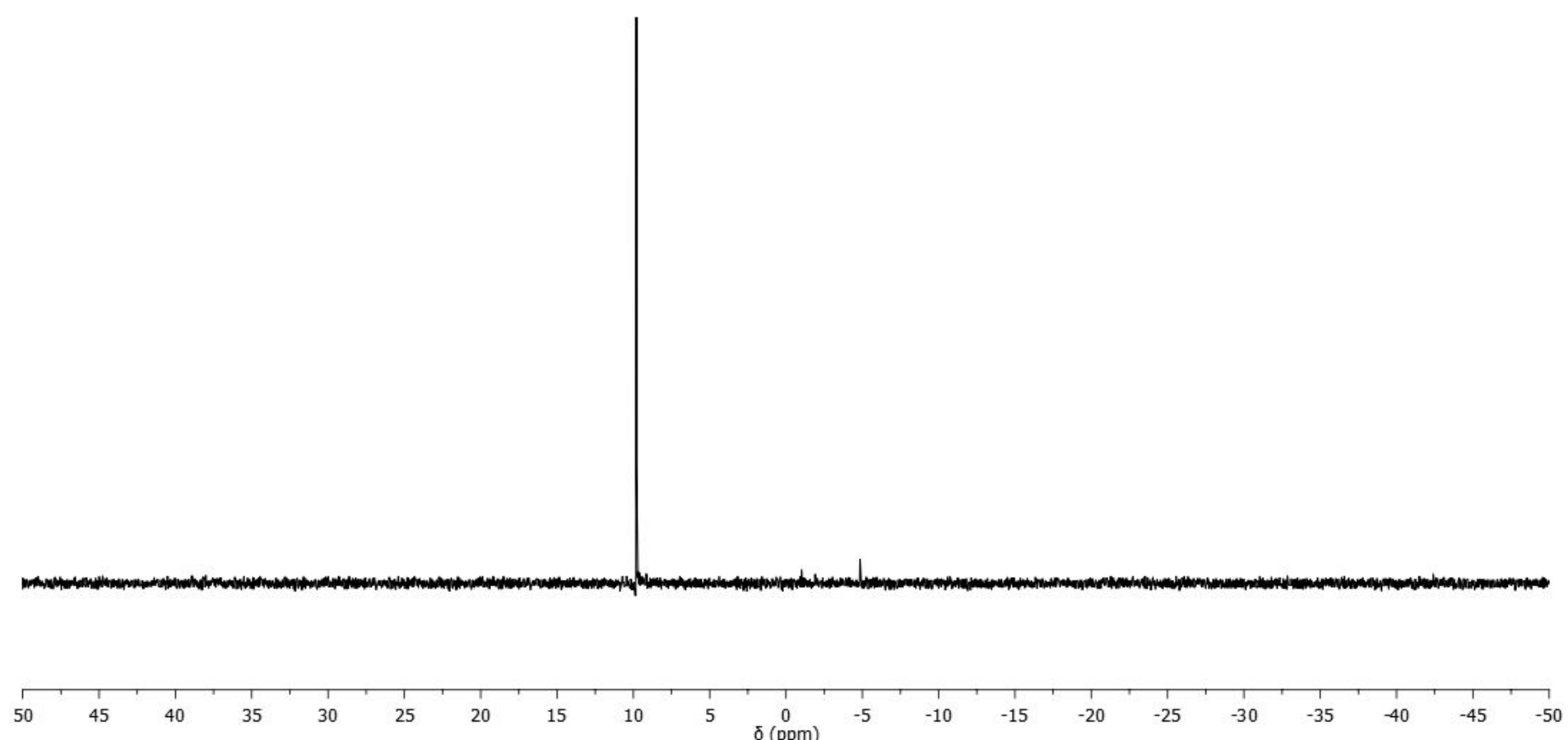

Figure S57. ${ }^{31} \mathrm{P}$ NMR spectrum of $\mathbf{3 n}$. 
Thiophene phosphonates

${ }^{1} \mathrm{H} 400 \mathrm{MHz}, \mathrm{CDCl}_{3}$

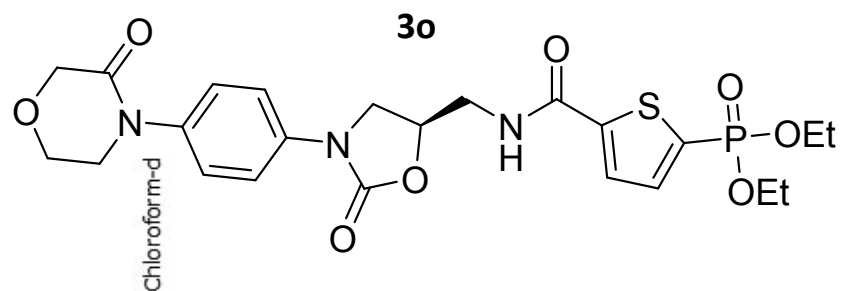

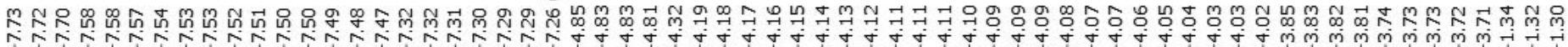

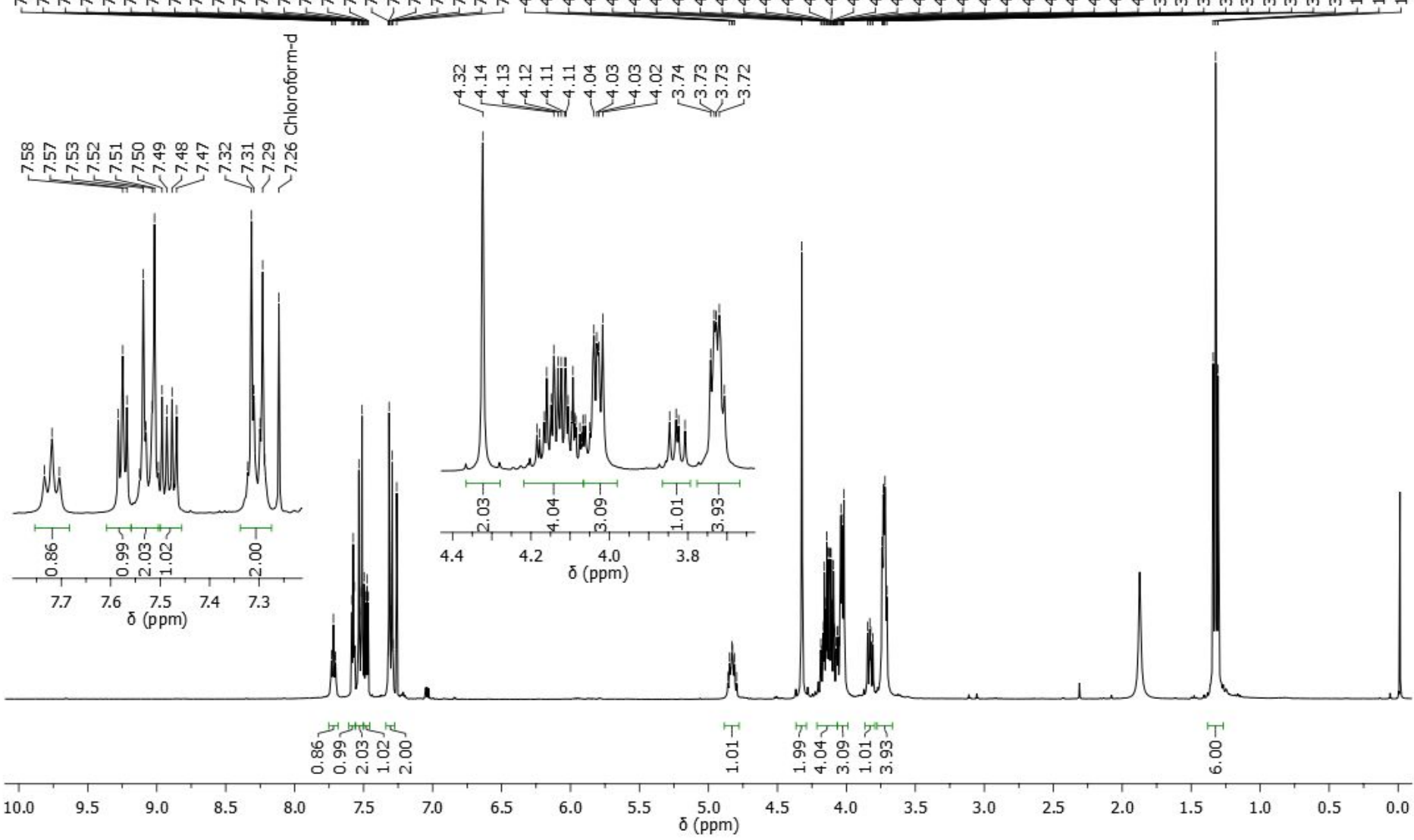

Figure S58. ${ }^{1} \mathrm{H}$ NMR spectrum of 30. 
${ }^{13} \mathrm{C}_{101 \mathrm{MHz}}, \mathrm{CDCl}_{3}$

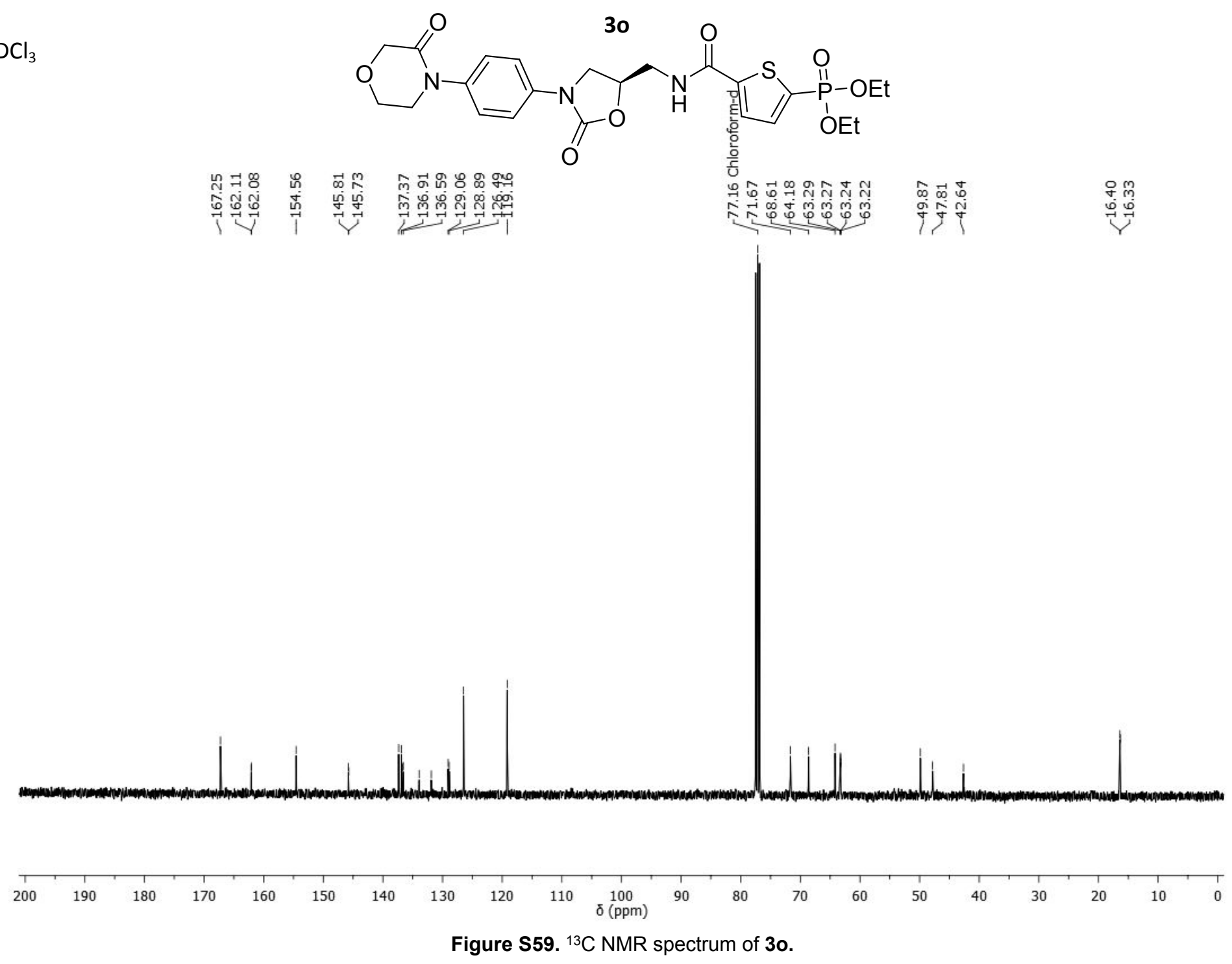


${ }^{31} \mathrm{P} 162 \mathrm{MHz}, \mathrm{CDCl}_{3}$
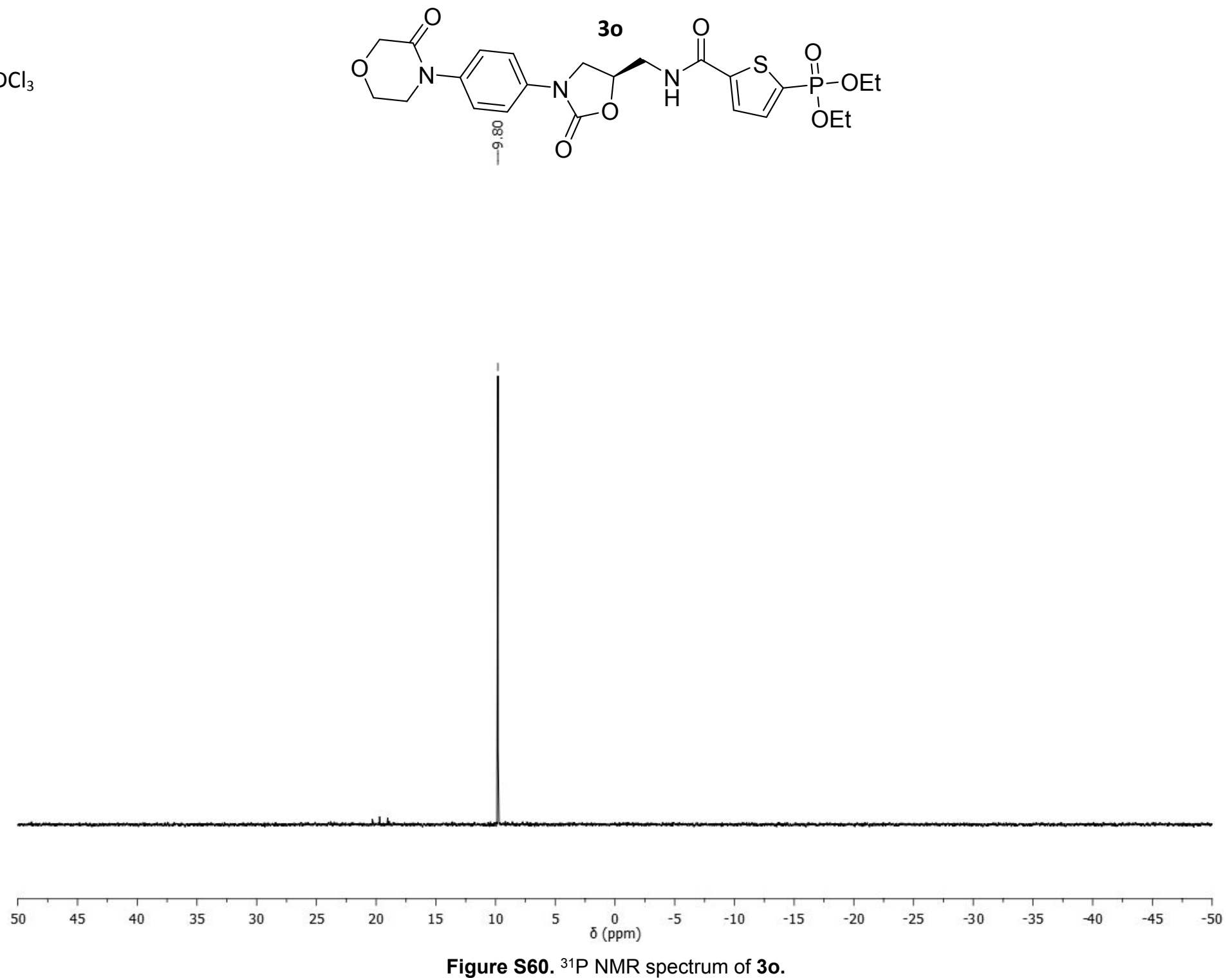
Furan phosphonates

${ }^{1} \mathrm{H} 400 \mathrm{MHz}, \mathrm{CDCl}_{3}$

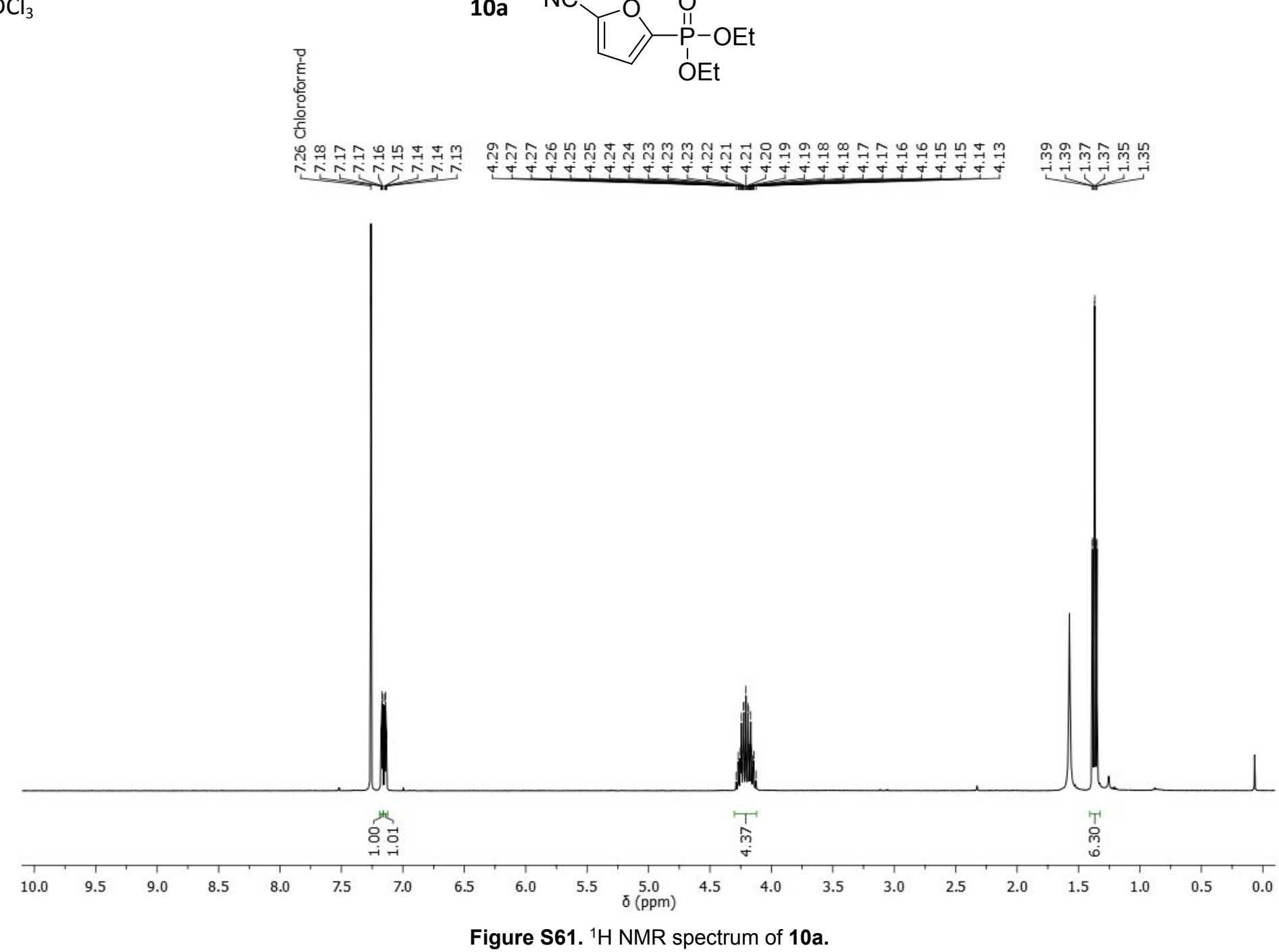


Furan phosphonates

${ }^{13} \mathrm{C} 101 \mathrm{MHz}, \mathrm{CDCl}_{3}$
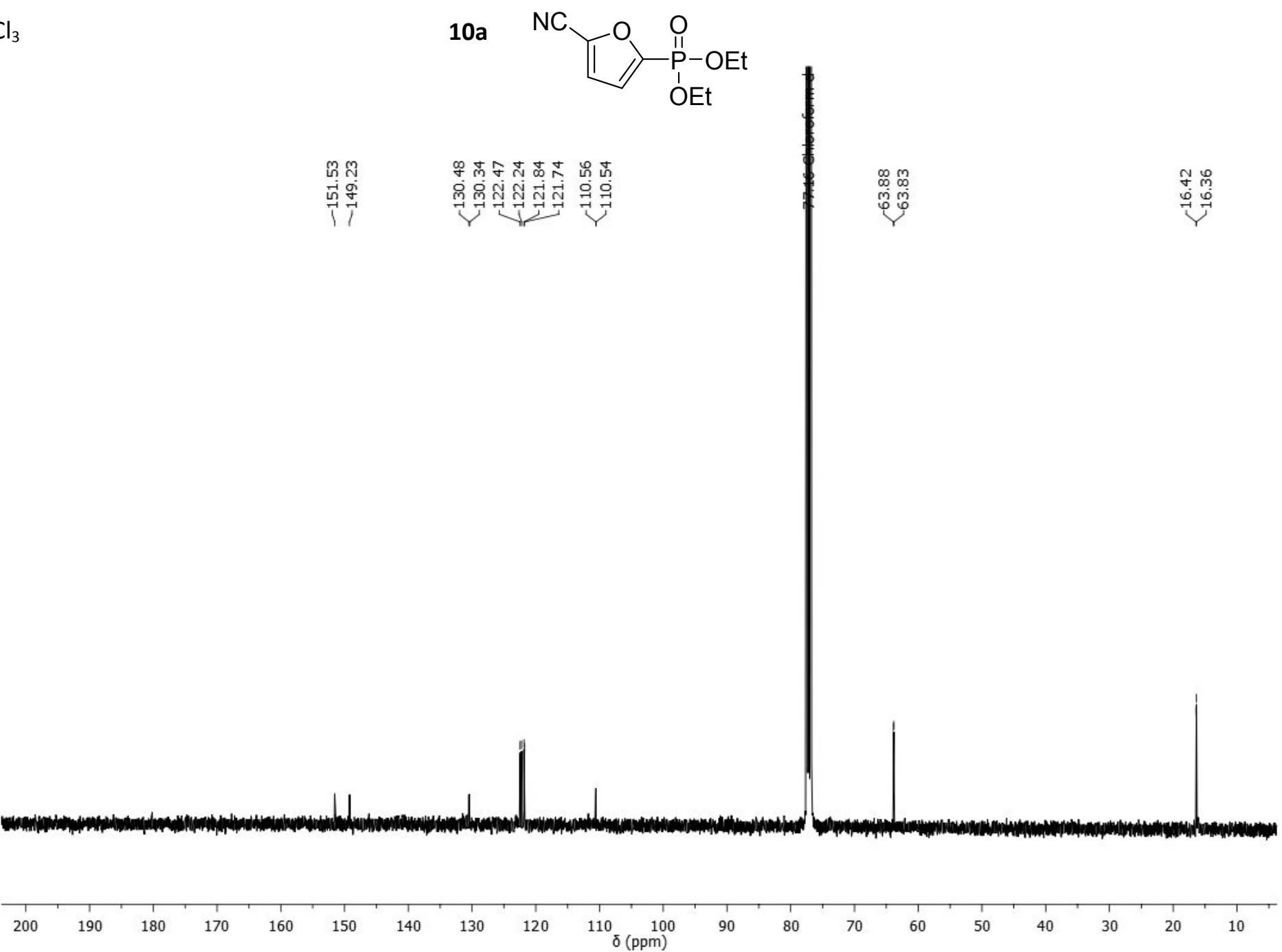

Figure S62. ${ }^{13} \mathrm{C}$ NMR spectrum of $10 \mathrm{a}$ 
Furan phosphonates

${ }^{31} \mathrm{P} 162 \mathrm{MHz}, \mathrm{CDCl}_{3}$

$10 a$
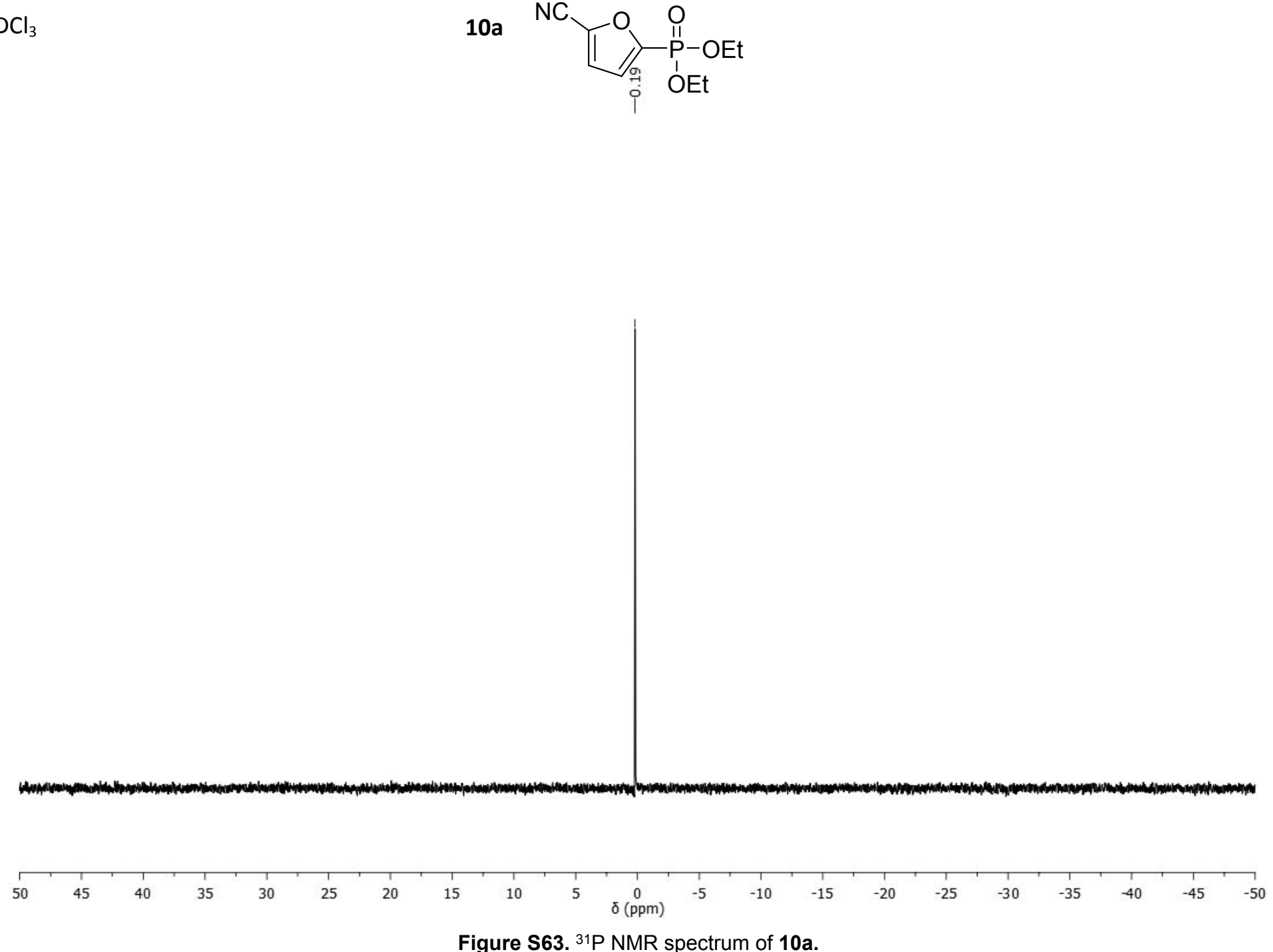

Figure S63. ${ }^{31} \mathrm{P}$ NMR spectrum of $10 a$. 
Furan phosphonates

${ }^{1} \mathrm{H} 400 \mathrm{MHz}, \mathrm{CDCl}_{3}$

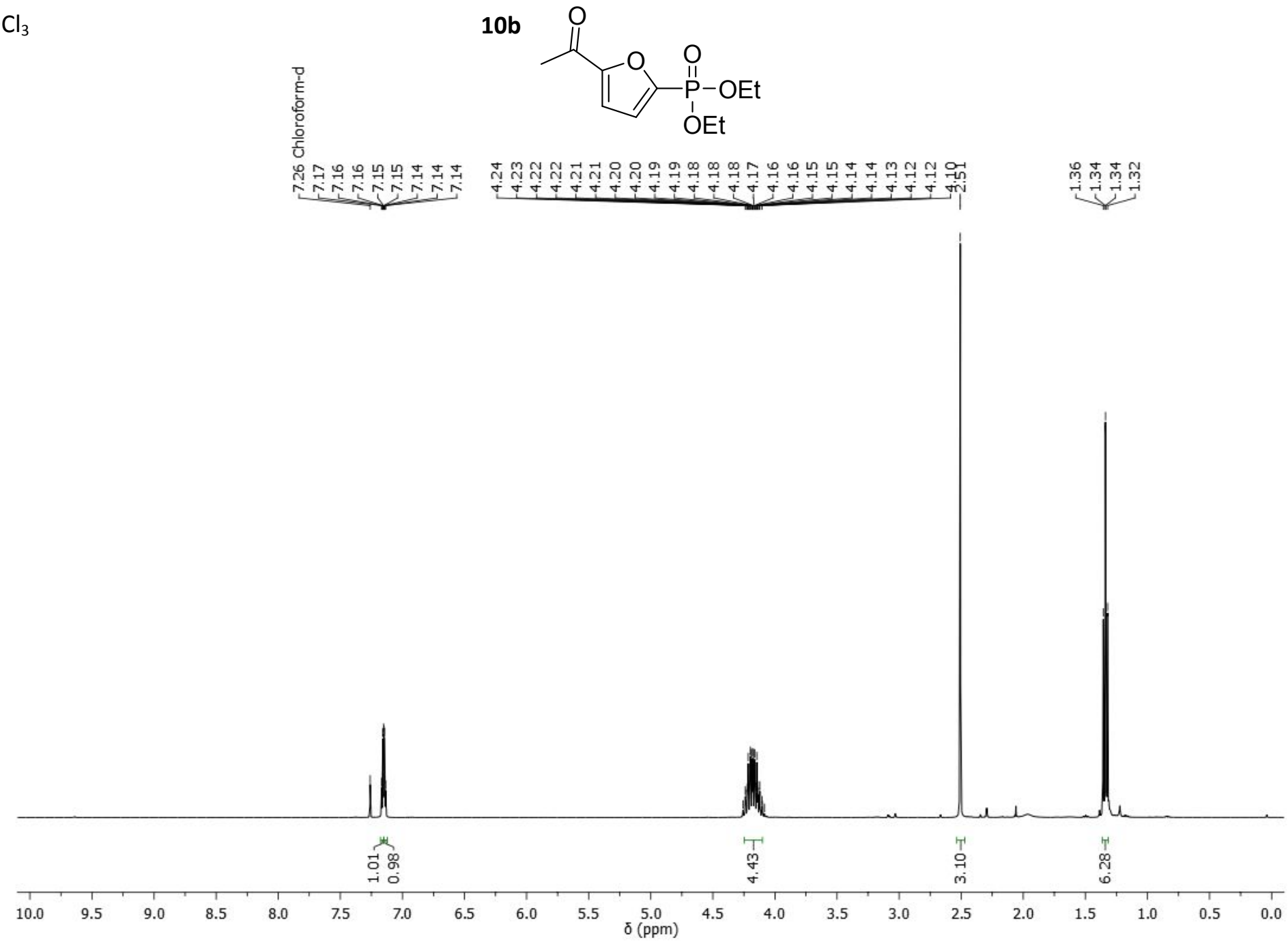

Figure S64. ${ }^{1} \mathrm{H}$ NMR spectrum of $10 \mathrm{~b}$. 
Furan phosphonates

${ }^{13} \mathrm{C} 101 \mathrm{MHz}, \mathrm{CDCl}_{3}$

$10 \mathrm{~b}$

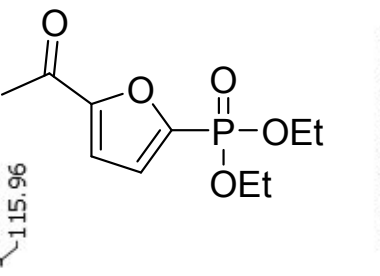

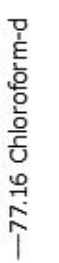

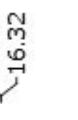

200

$190 \quad 180$

$180 \quad 170$

$160 \quad 150$

140

Figure S65. ${ }^{13} \mathrm{C}$ NMR spectrum of $10 \mathrm{~b}$. 


\section{Furan phosphonates}

${ }^{31} \mathrm{P} 162 \mathrm{MHz}, \mathrm{CDCl}_{3}$

$10 \mathrm{~b}$
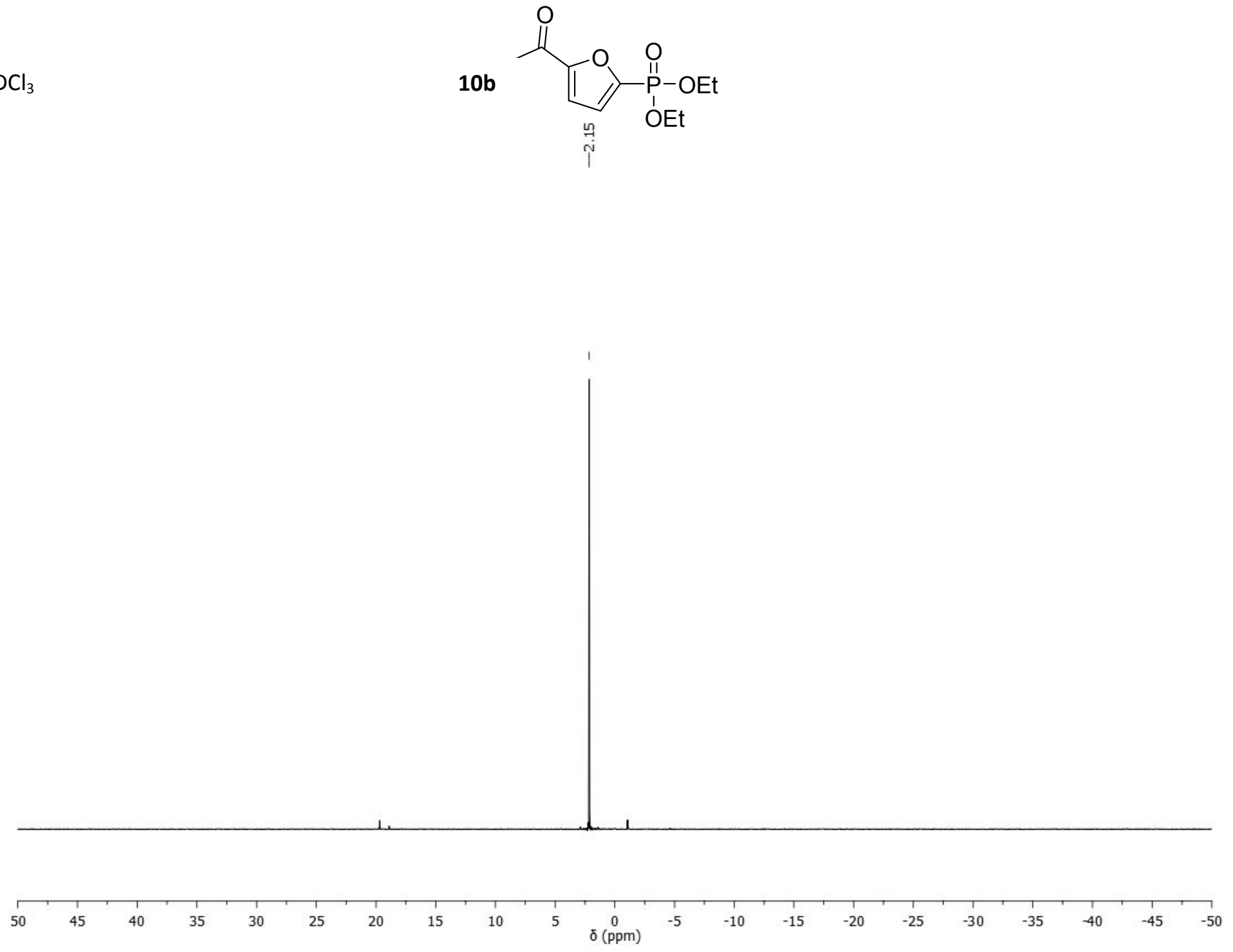

Figure S66. ${ }^{31} \mathrm{P}$ NMR spectrum of $10 \mathrm{~b}$. 
Furan phosphonates

${ }^{1} \mathrm{H} 400 \mathrm{MHz}, \mathrm{CDCl}_{3}$

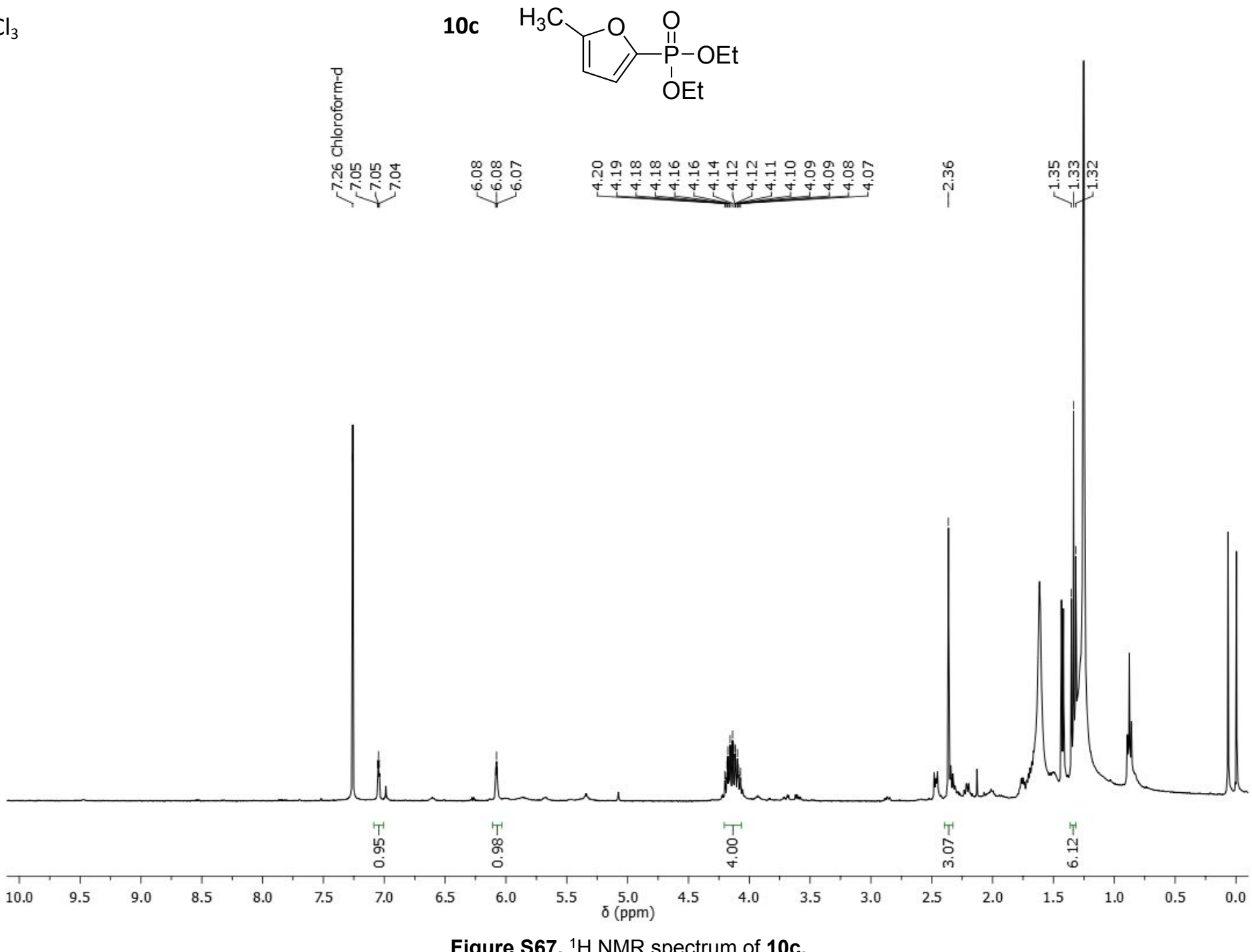

Figure S67. ${ }^{1} \mathrm{H}$ NMR spectrum of $10 \mathrm{c}$. 
${ }^{13} \mathrm{C} 101 \mathrm{MHz}, \mathrm{CDCl}_{3}$

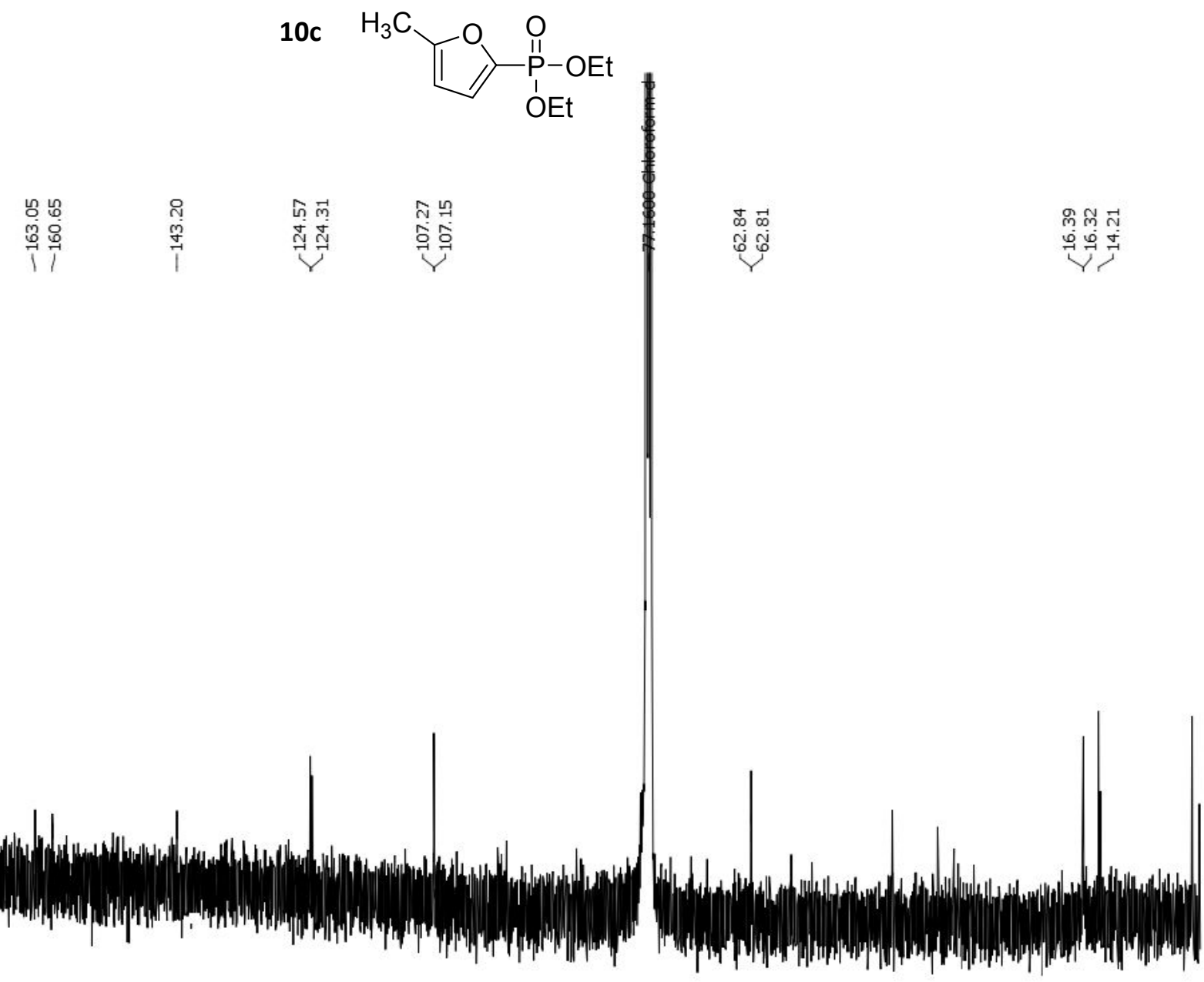

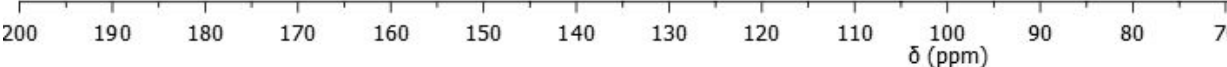

Figure S68. ${ }^{13} \mathrm{C}$ NMR spectrum of $10 \mathrm{c}$. 
${ }^{31} \mathrm{P} 162 \mathrm{MHz}, \mathrm{CDCl}_{3}$

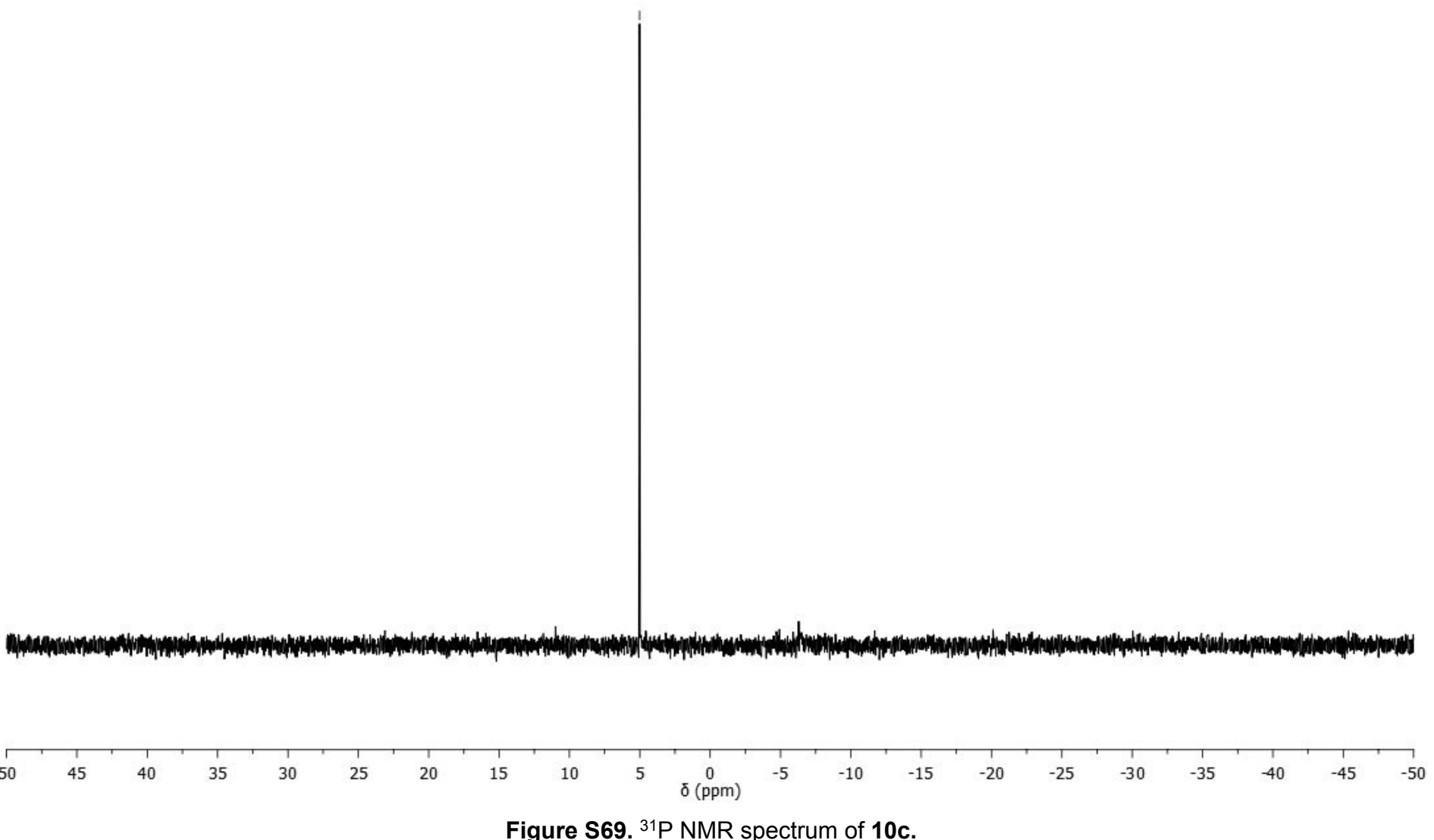

10c

$$
\stackrel{\substack{\mathrm{O} \\ \stackrel{\mathrm{O}}{\mathrm{O}}-\mathrm{OEt}}}{\mathrm{H}_{3} \mathrm{C}}
$$

Figure S69. ${ }^{31} \mathrm{P}$ NMR spectrum of 10c. 


\section{Pyrrole phosphonate}

${ }^{1} \mathrm{H} 400 \mathrm{MHz}, \mathrm{CDCl}_{3}$

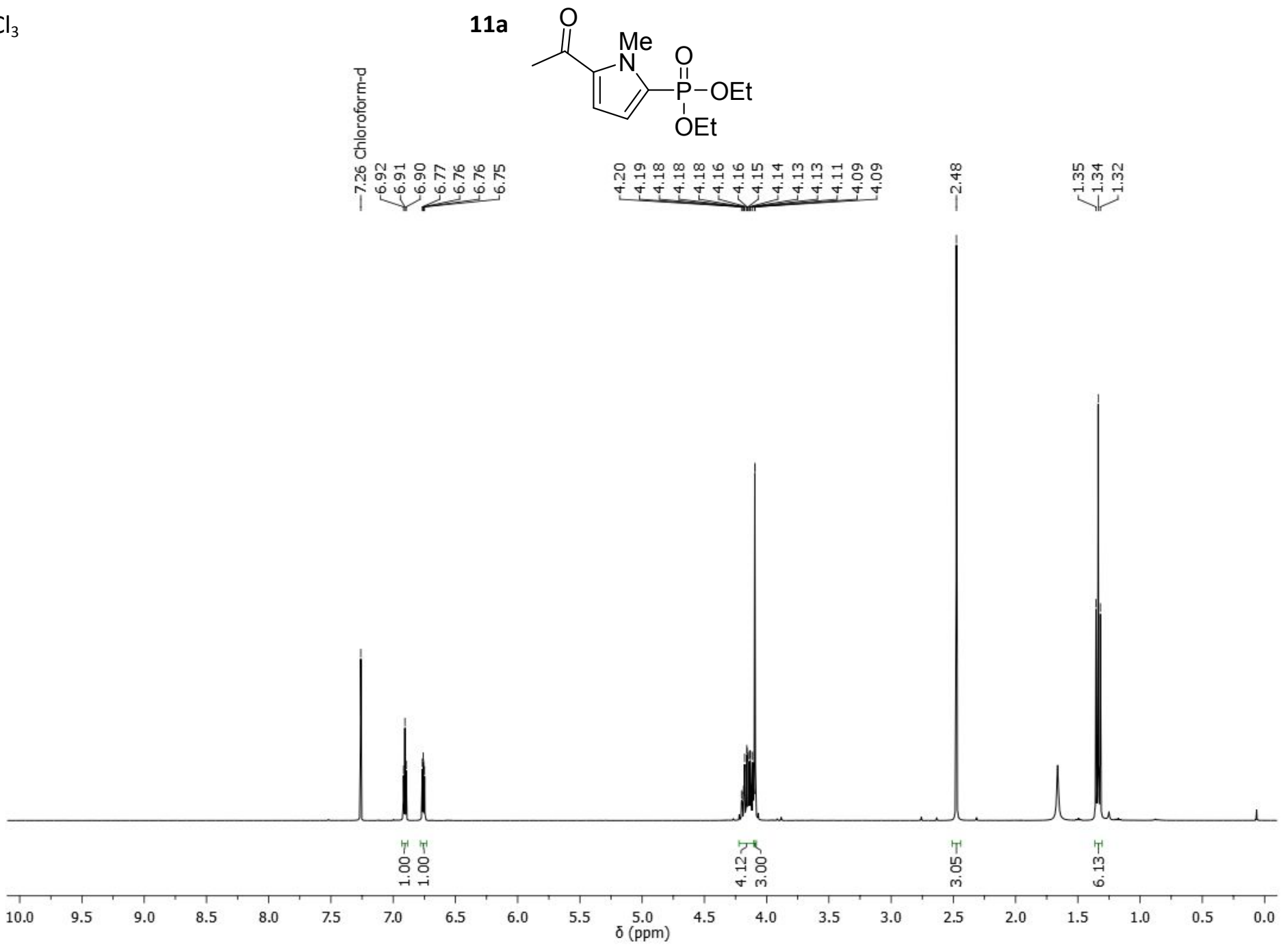

Figure S70. ${ }^{1} \mathrm{H}$ NMR spectrum of $11 \mathrm{a}$. 


\section{Pyrrole phosphonate}

${ }^{13} \mathrm{C} 101 \mathrm{MHz}, \mathrm{CDCl}_{3}$

$11 a$

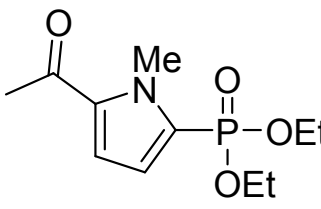

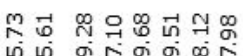

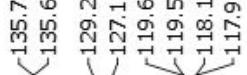

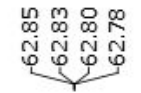

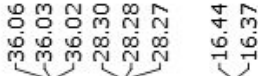

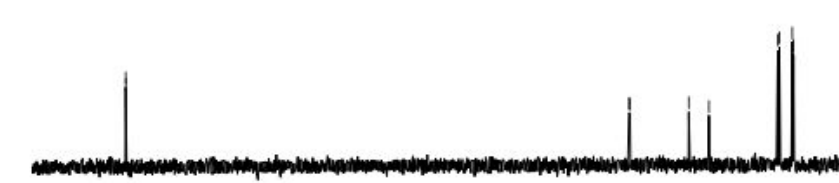

$200 \quad 190 \quad 180 \quad 170 \quad 160$

Figure S71. ${ }^{13} \mathrm{C}$ NMR spectrum of $11 \mathrm{a}$ 


\section{Pyrrole phosphonate}

${ }^{31} \mathrm{P} 162 \mathrm{MHz}, \mathrm{CDCl}_{3}$
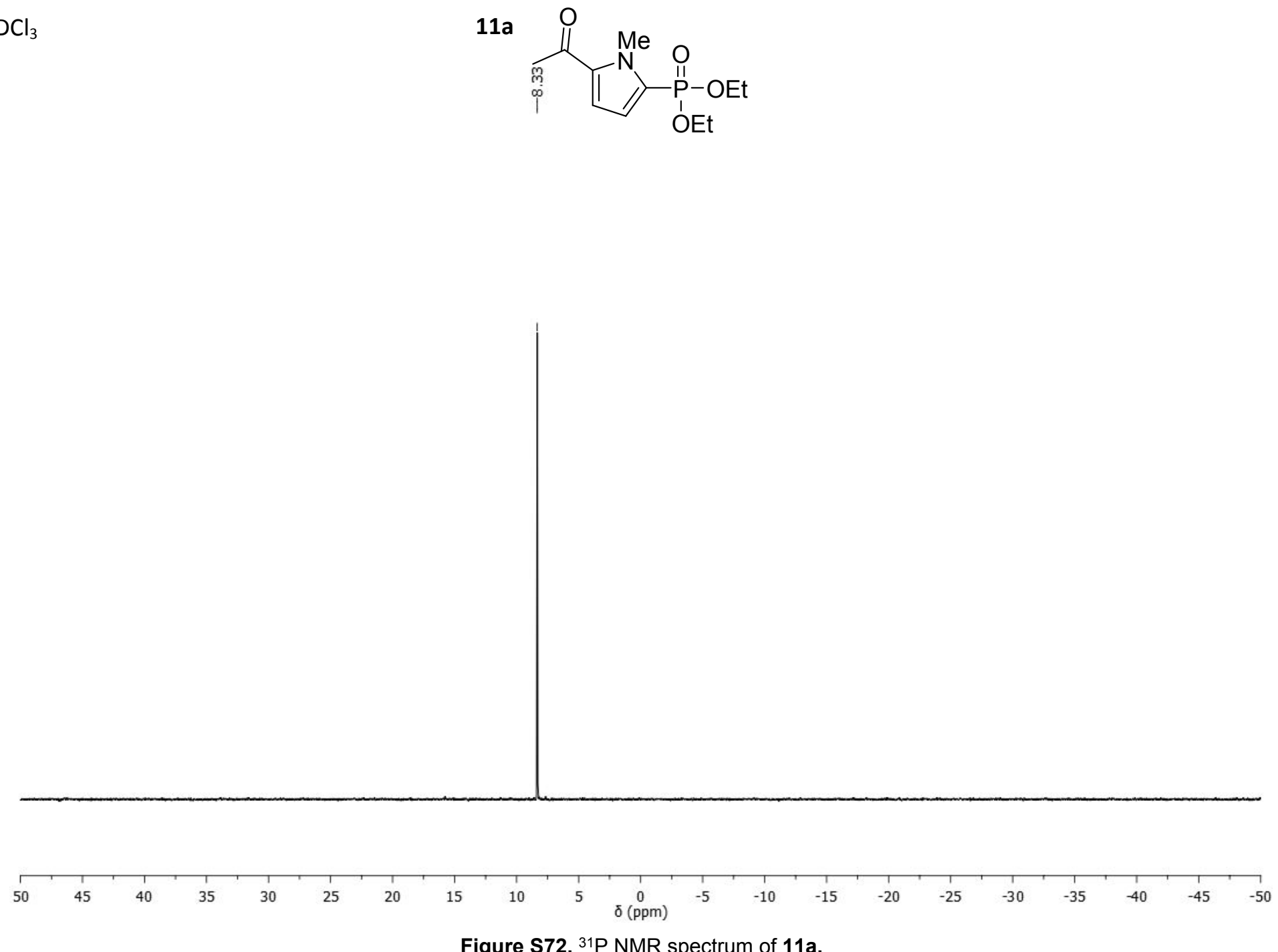

Figure S72. ${ }^{31} \mathrm{P}$ NMR spectrum of 11a. 
Selenophene phosphonate

${ }^{1} \mathrm{H} 400 \mathrm{MHz}, \mathrm{CDCl}_{3}$

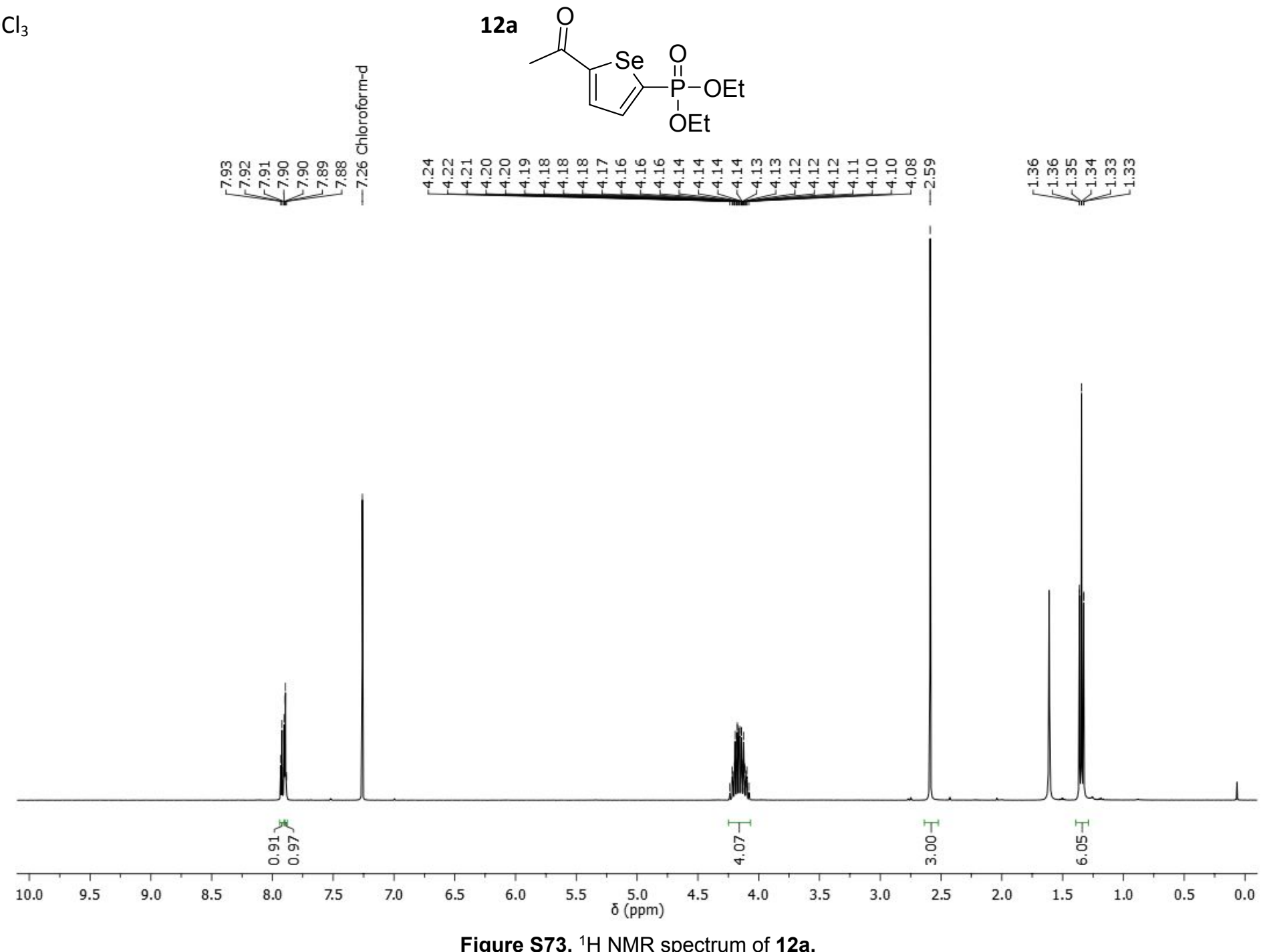

Figure S73. ${ }^{1} \mathrm{H}$ NMR spectrum of $12 \mathrm{a}$. 
Selenophene phosphonate

${ }^{13} \mathrm{C} 101 \mathrm{MHz}, \mathrm{CDCl}_{3}$

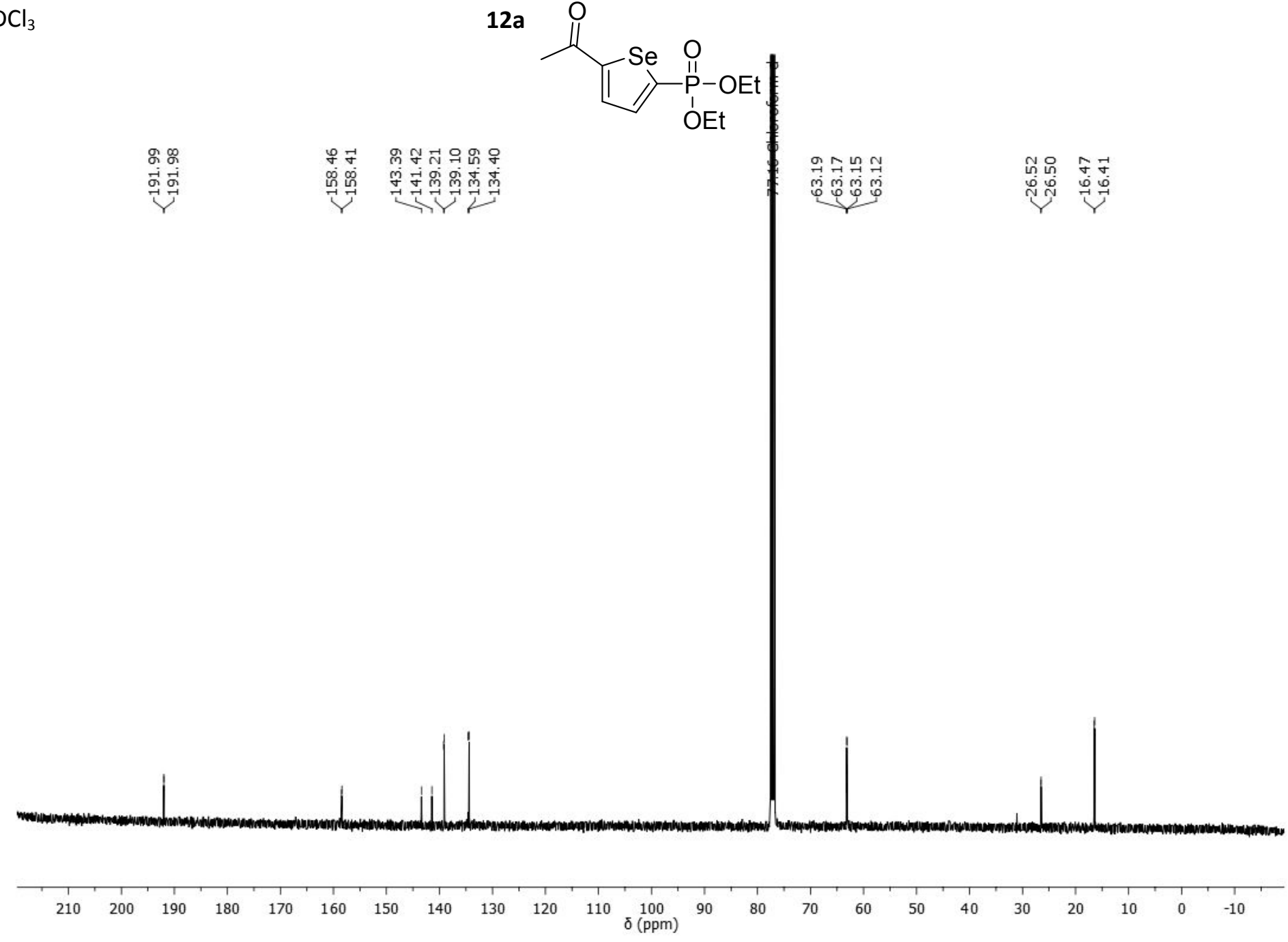

Figure $574 .{ }^{13} \mathrm{C}$ NMR spectrum of $12 \mathrm{a}$. 
${ }^{31} \mathrm{P} 162 \mathrm{MHz}, \mathrm{CDCl}_{3}$

$12 a$
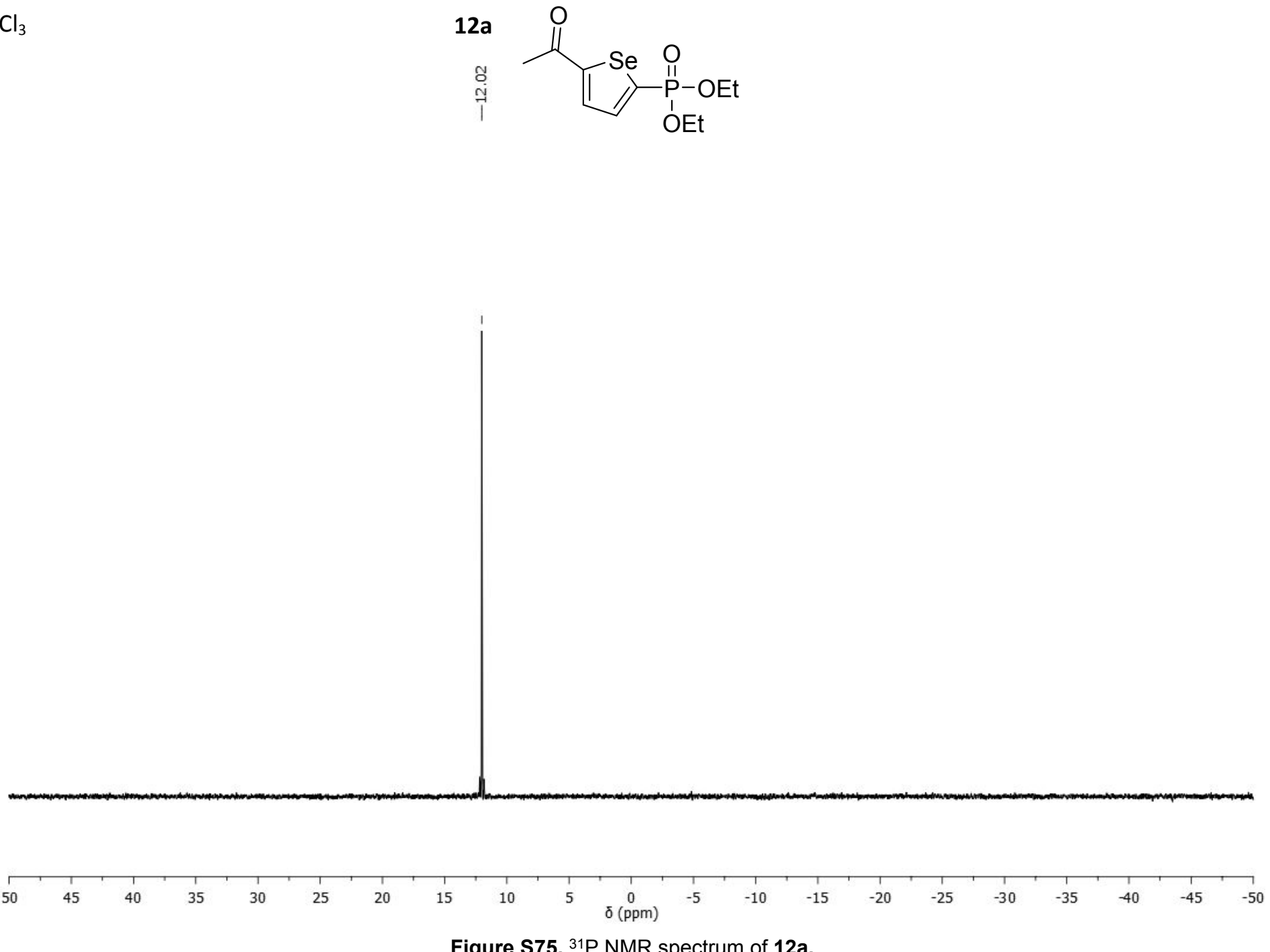

Figure S75. ${ }^{31} \mathrm{P}$ NMR spectrum of $12 \mathrm{a}$. 
${ }^{1} \mathrm{H} 400 \mathrm{MHz}, \mathrm{CDCl}_{3}$

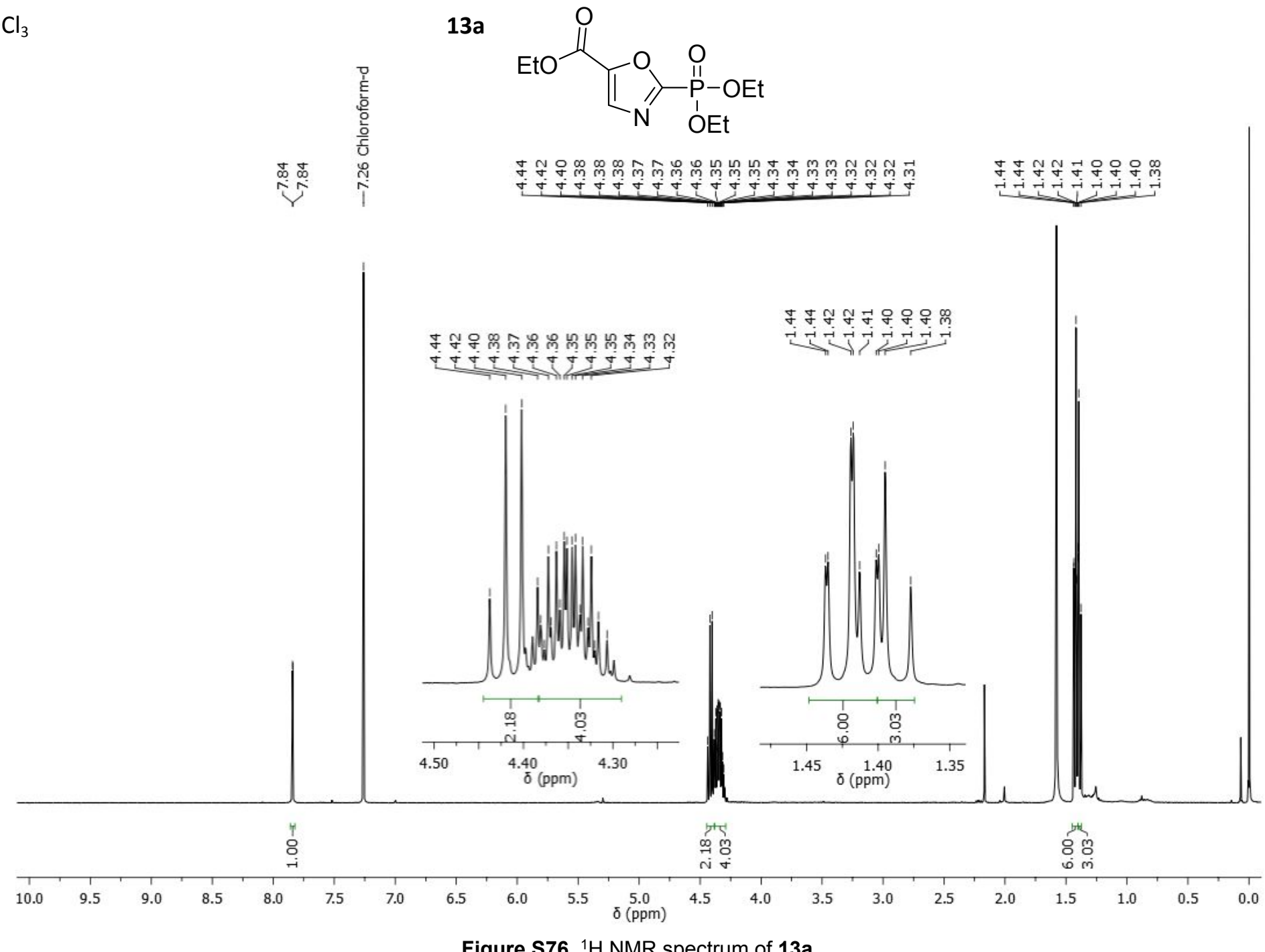

Figure S76. ${ }^{1} \mathrm{H}$ NMR spectrum of $13 a$. 
${ }^{13} \mathrm{C} 101 \mathrm{MHz}, \mathrm{CDCl}_{3}$

$13 a$

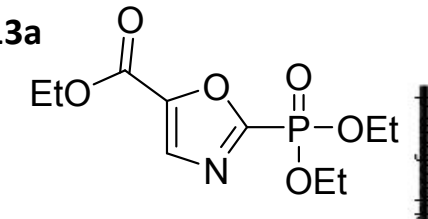

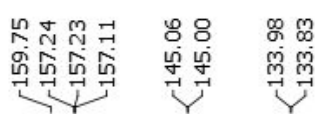

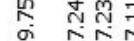

角鱼望

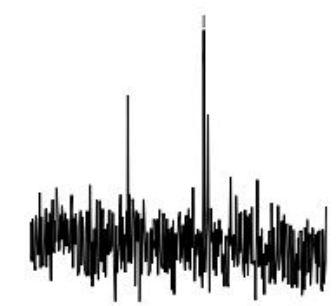

${ }^{160} \delta(\mathrm{ppm}) \quad 155$

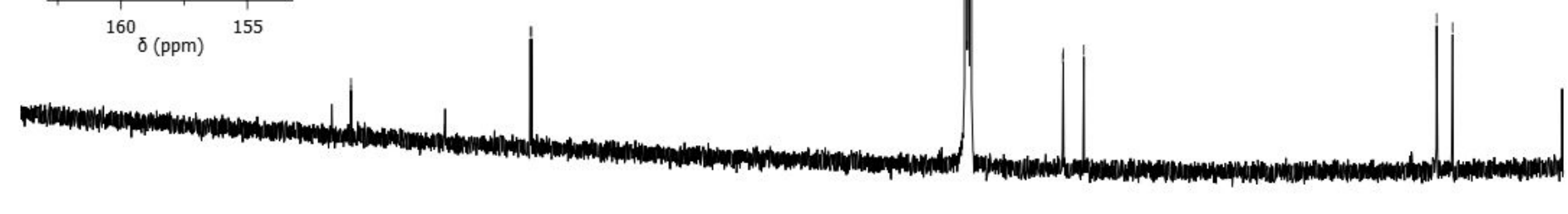

$\begin{array}{llllllll}200 & 190 & 180 & 170 & 160 & 150 & 140 & 130\end{array}$

Figure S77. ${ }^{13} \mathrm{C}$ NMR spectrum of $13 a$. 


\section{Oxazole phosphonate}

${ }^{31} \mathrm{P} 162 \mathrm{MHz}, \mathrm{CDCl}_{3}$

$13 a$

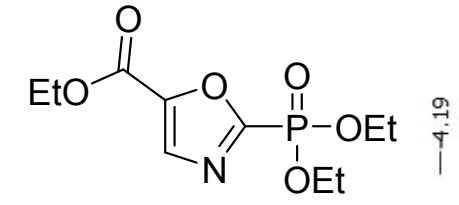

1.

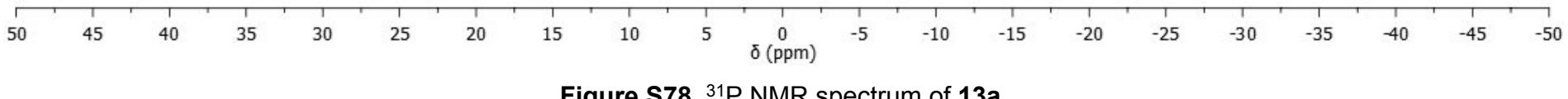

Figure S78. ${ }^{31} \mathrm{P}$ NMR spectrum of $13 \mathrm{a}$. 
${ }^{1} \mathrm{H} 400 \mathrm{MHz}, \mathrm{CDCl}_{3}$

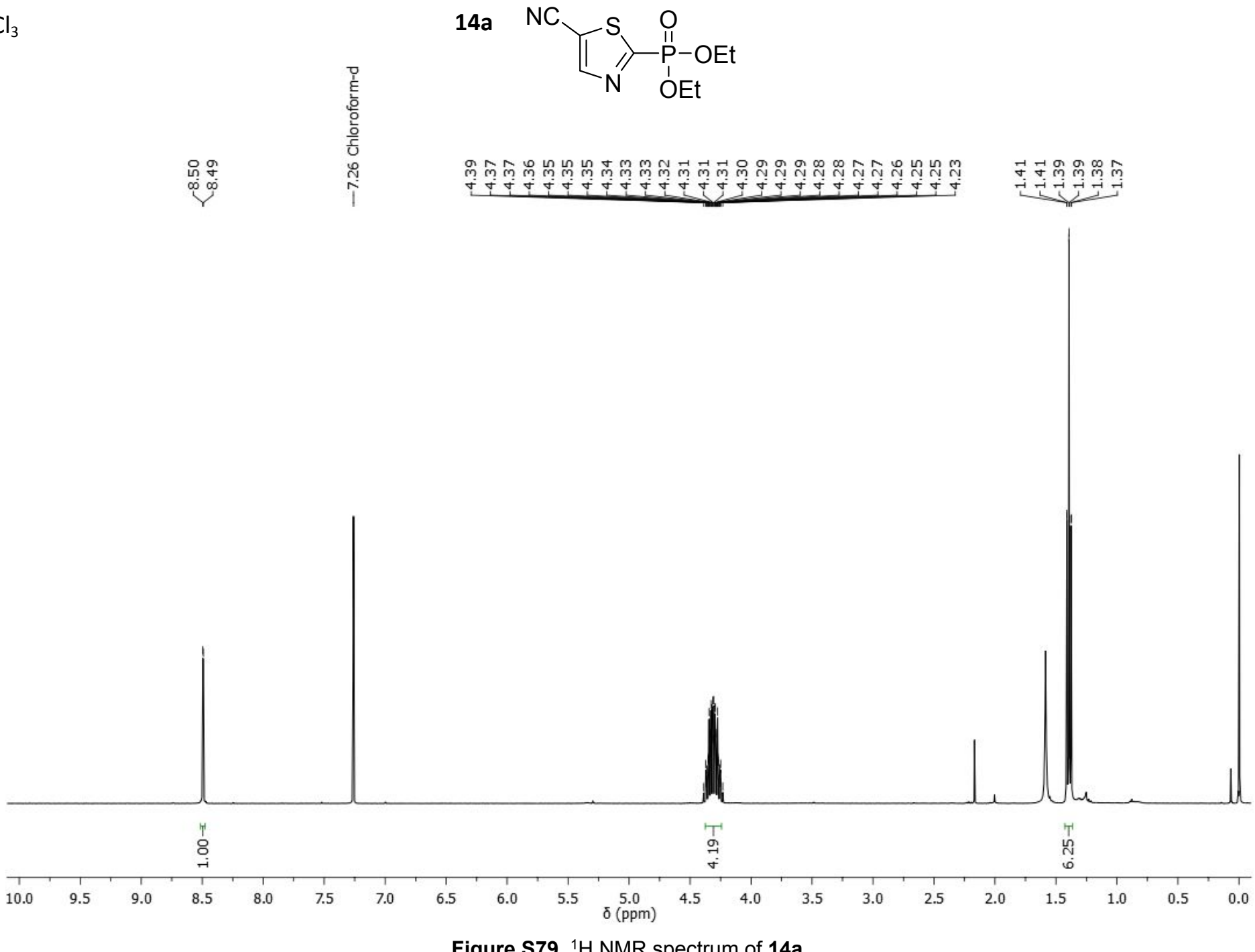


${ }^{13} \mathrm{C} 101 \mathrm{MHz}, \mathrm{CDCl}_{3}$

$14 a$
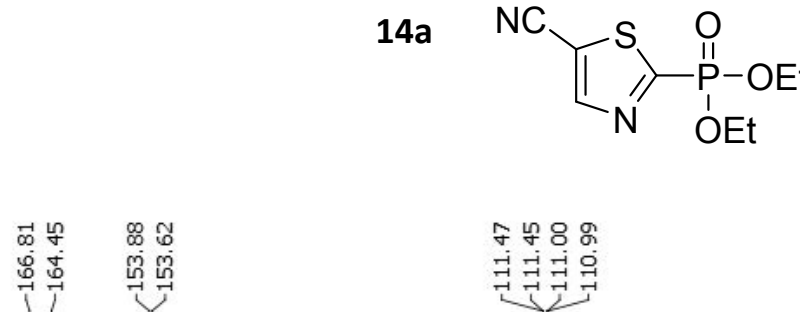

\&

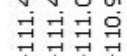

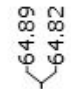

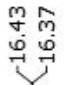

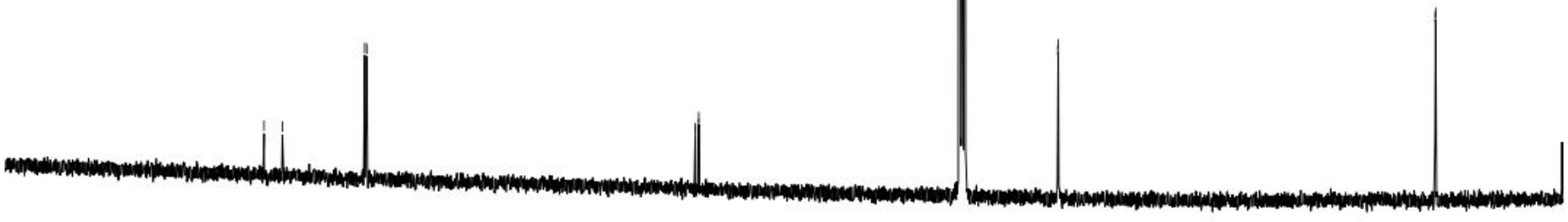

$\begin{array}{llllllllllllll}200 & 190 & 180 & 170 & 160 & 150 & 140 & 130 & 120 & 110 & \begin{array}{c}100 \\ \delta(\mathrm{ppm})\end{array} & 90 & 80 & 7\end{array}$

Figure S80. ${ }^{13} \mathrm{C}$ NMR spectrum of $14 a$. 
${ }^{31} \mathrm{P} 162 \mathrm{MHz}, \mathrm{CDCl}_{3}$

$14 a \stackrel{N C}{\text { II }}$

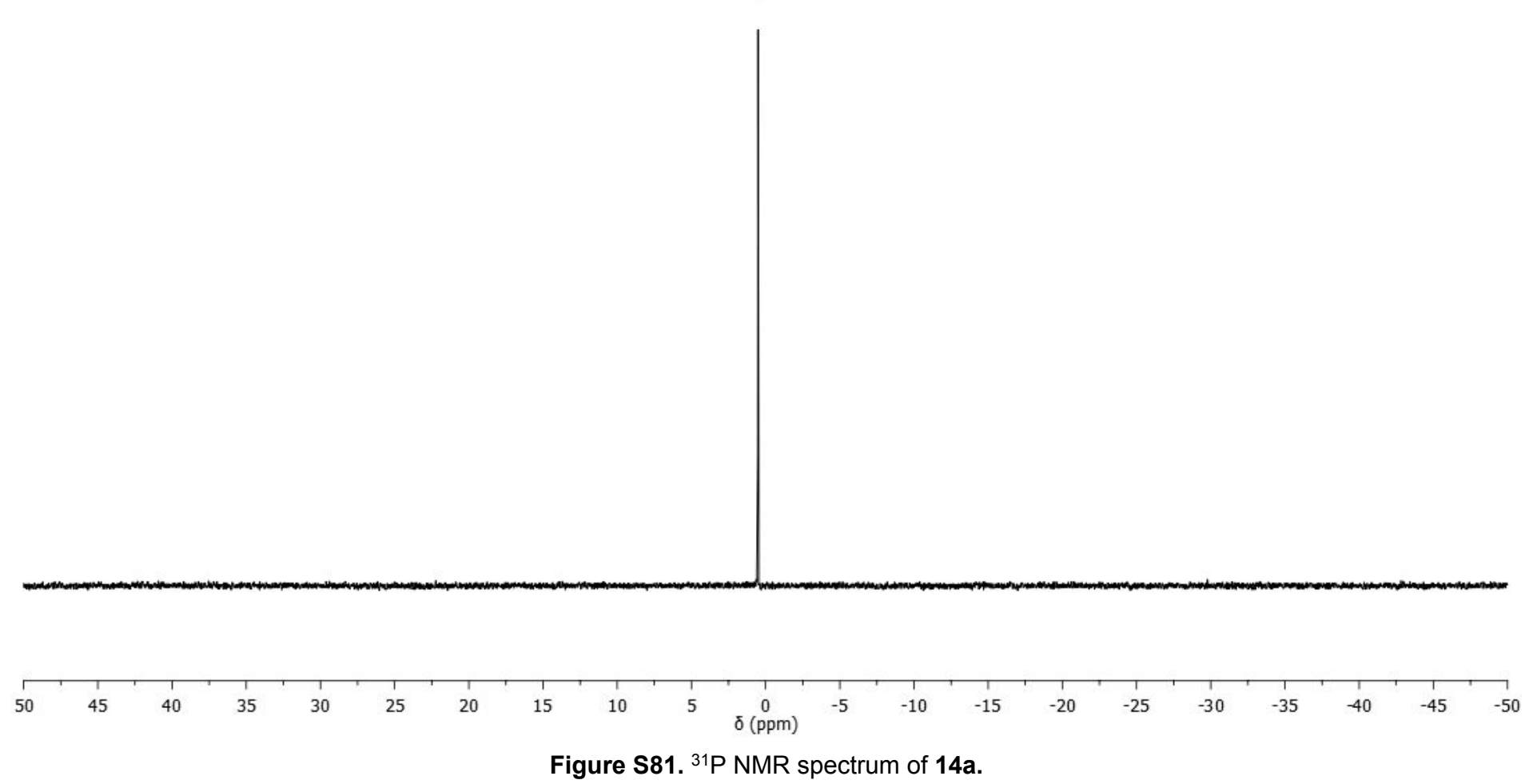


${ }^{1} \mathrm{H} 400 \mathrm{MHz}, \mathrm{CDCl}_{3}$

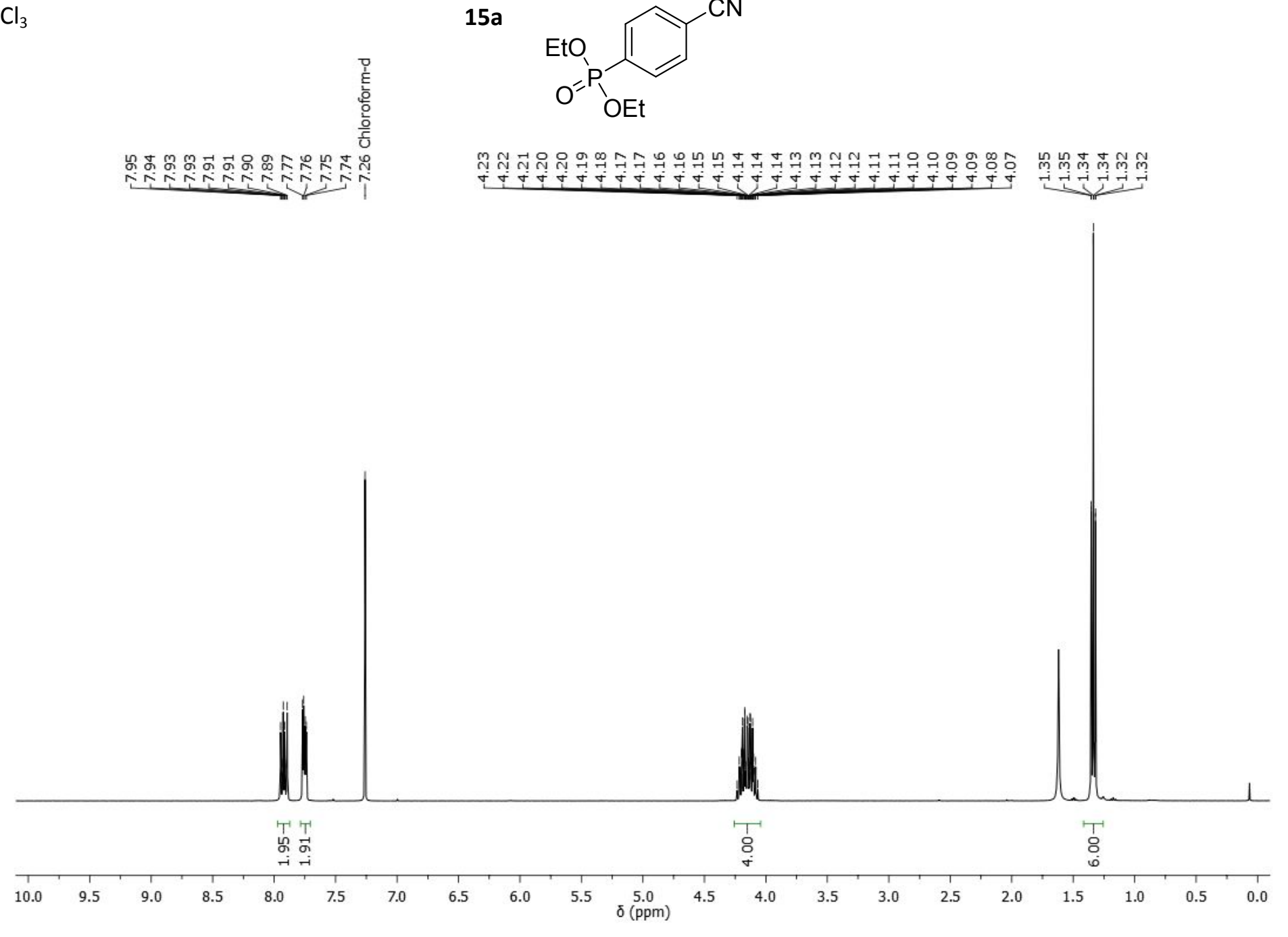

Figure S82. ${ }^{1} \mathrm{H}$ NMR spectrum of 15 a. 
Benzonitrile phosphonates

${ }^{13} \mathrm{C} 101 \mathrm{MHz}, \mathrm{CDCl}_{3}$

$15 a$

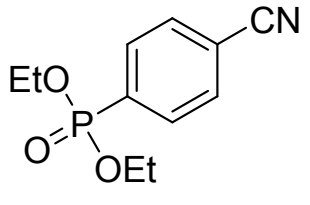

ํㅜㅇํำ

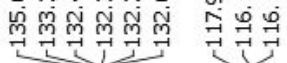

象

藏走

$\sqrt{0}$

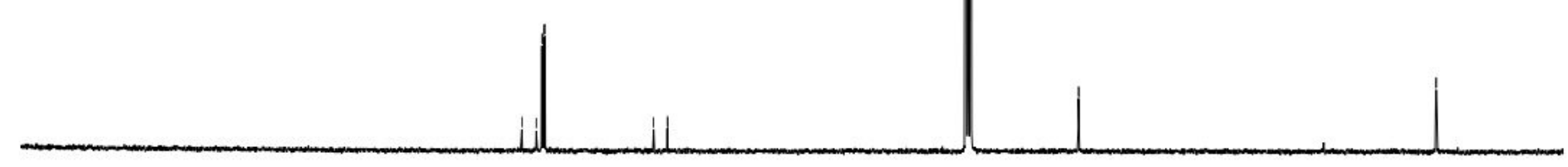

$200 \quad 190 \quad 180 \quad 170+160$

100
$\delta(\mathrm{ppm})$

Figure S83. ${ }^{13} \mathrm{C}$ NMR spectrum of $15 \mathrm{a}$. 
${ }^{1} \mathrm{H} 400 \mathrm{MHz}, \mathrm{CDCl}_{3}$

$15 b$

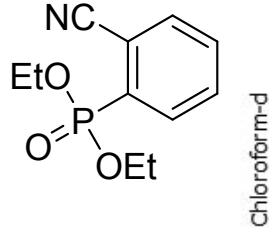

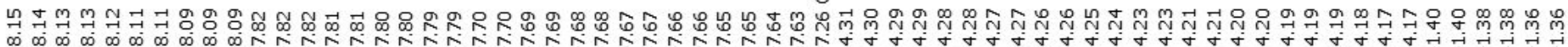

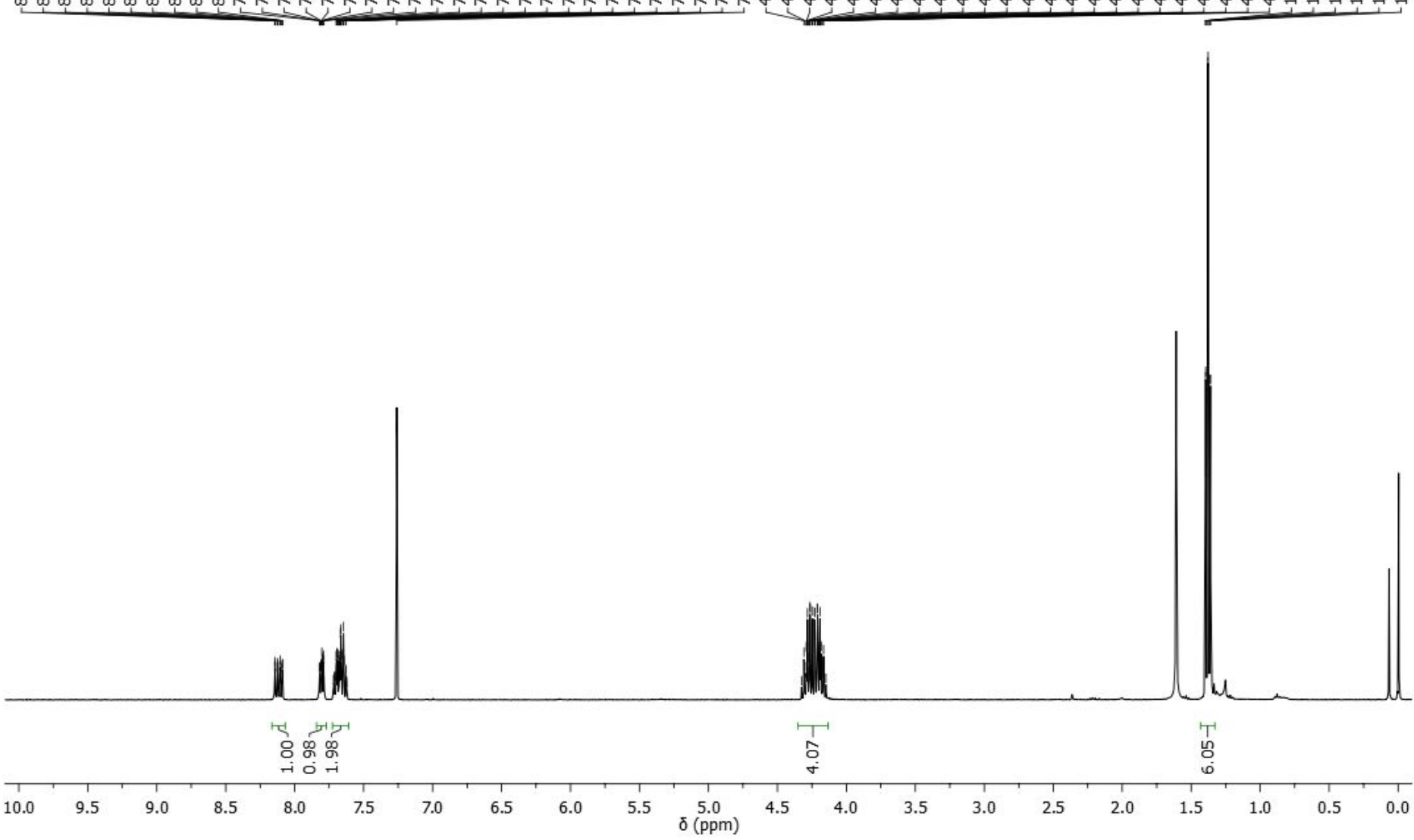

Figure S84. ${ }^{1} \mathrm{H}$ NMR spectrum of $15 b$. 
Benzonitrile phosphonates

${ }^{13} \mathrm{C} 101 \mathrm{MHz}, \mathrm{CDCl}_{3}$

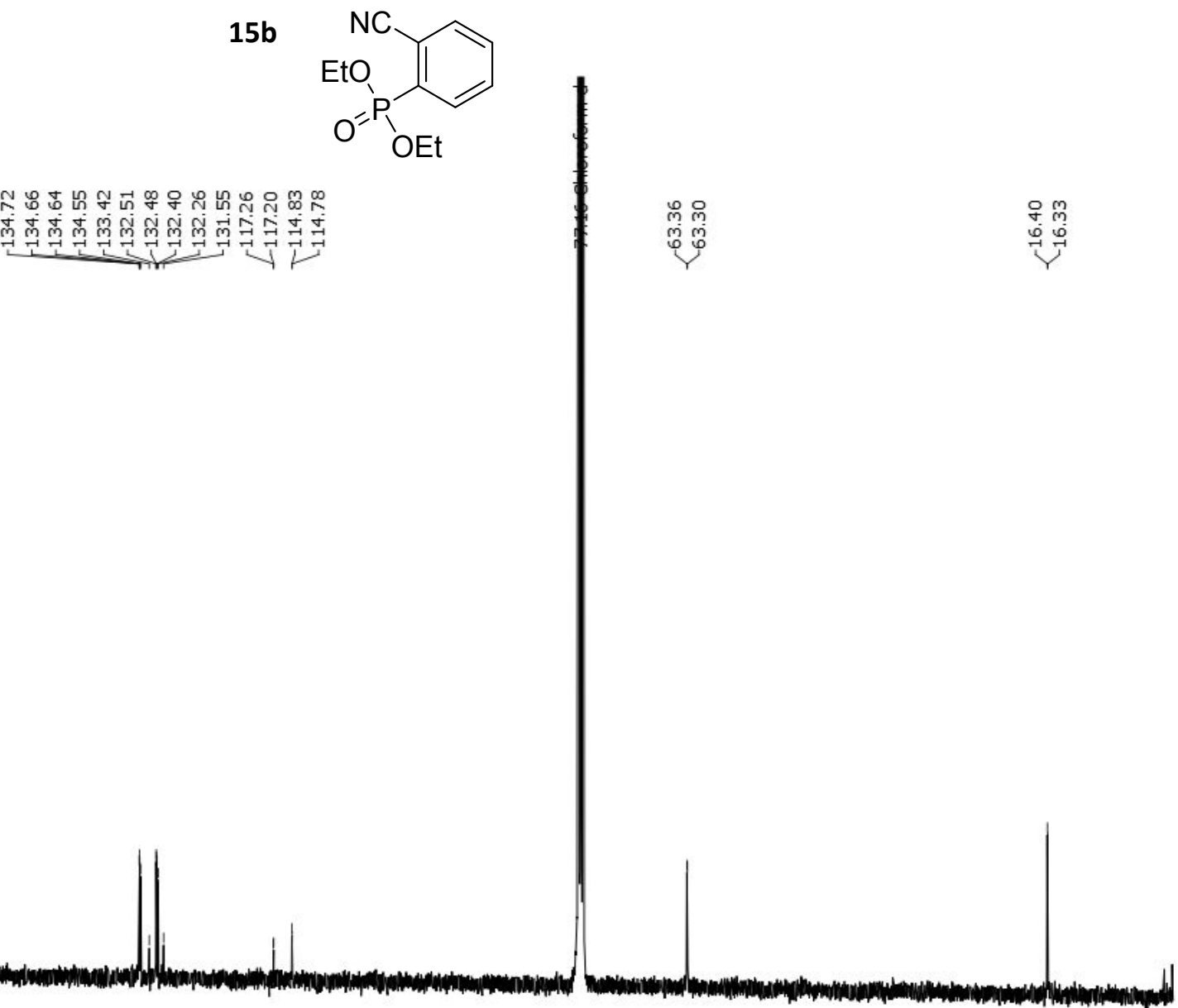

$\begin{array}{llllllll}200 & 190 & 180 & 170 & 160 & 150 & 140 & 130\end{array}$

Figure $585 .{ }^{13} \mathrm{C}$ NMR spectrum of $15 \mathrm{~b}$. 


\section{Quinoline phosphonates}

${ }^{1} \mathrm{H} 400 \mathrm{MHz}, \mathrm{CDCl}_{3}$

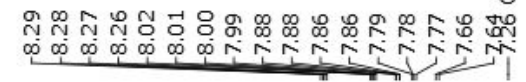

$15 c$

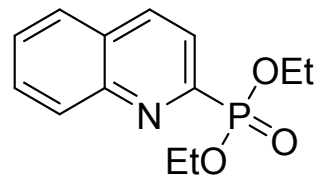

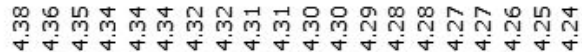

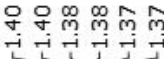

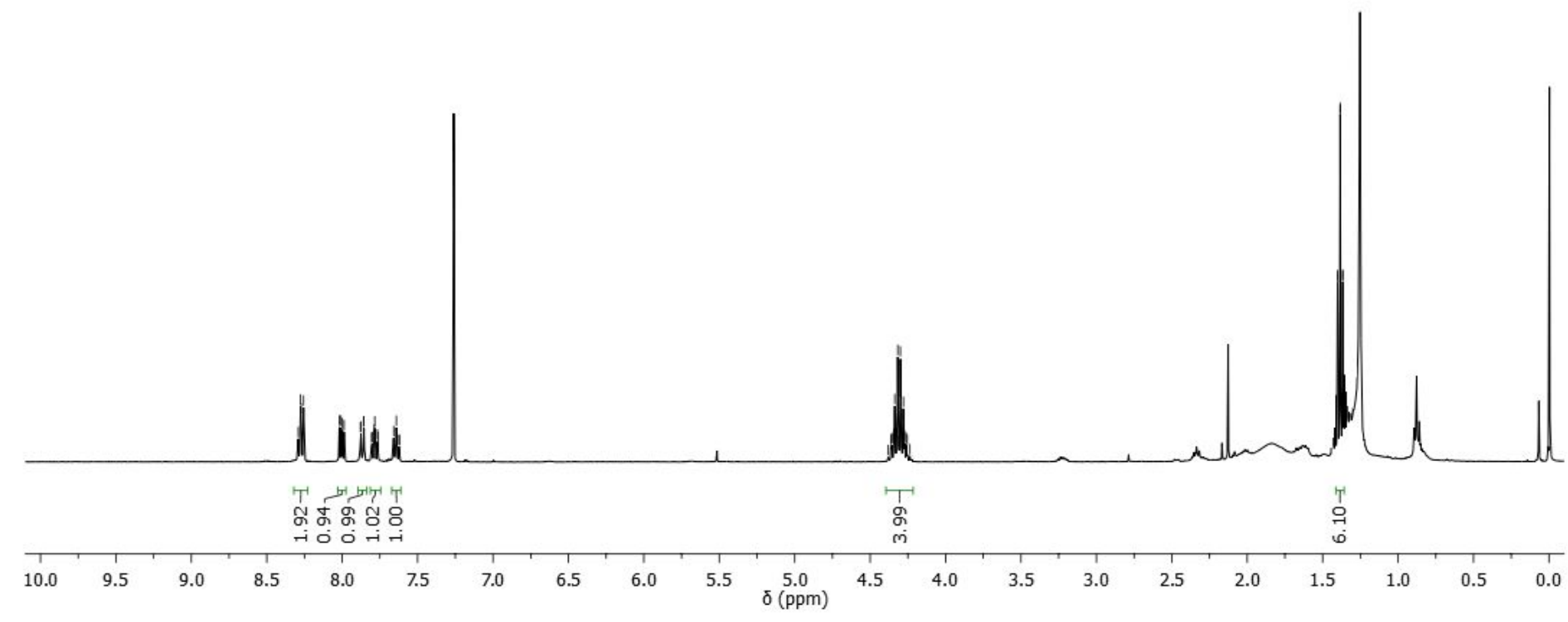

Figure S86. ${ }^{1} \mathrm{H}$ NMR spectrum of $15 \mathrm{c}$ 


\section{Quinoline phosphonates}

${ }^{1} \mathrm{H} 400 \mathrm{MHz}, \mathrm{CDCl}_{3}$

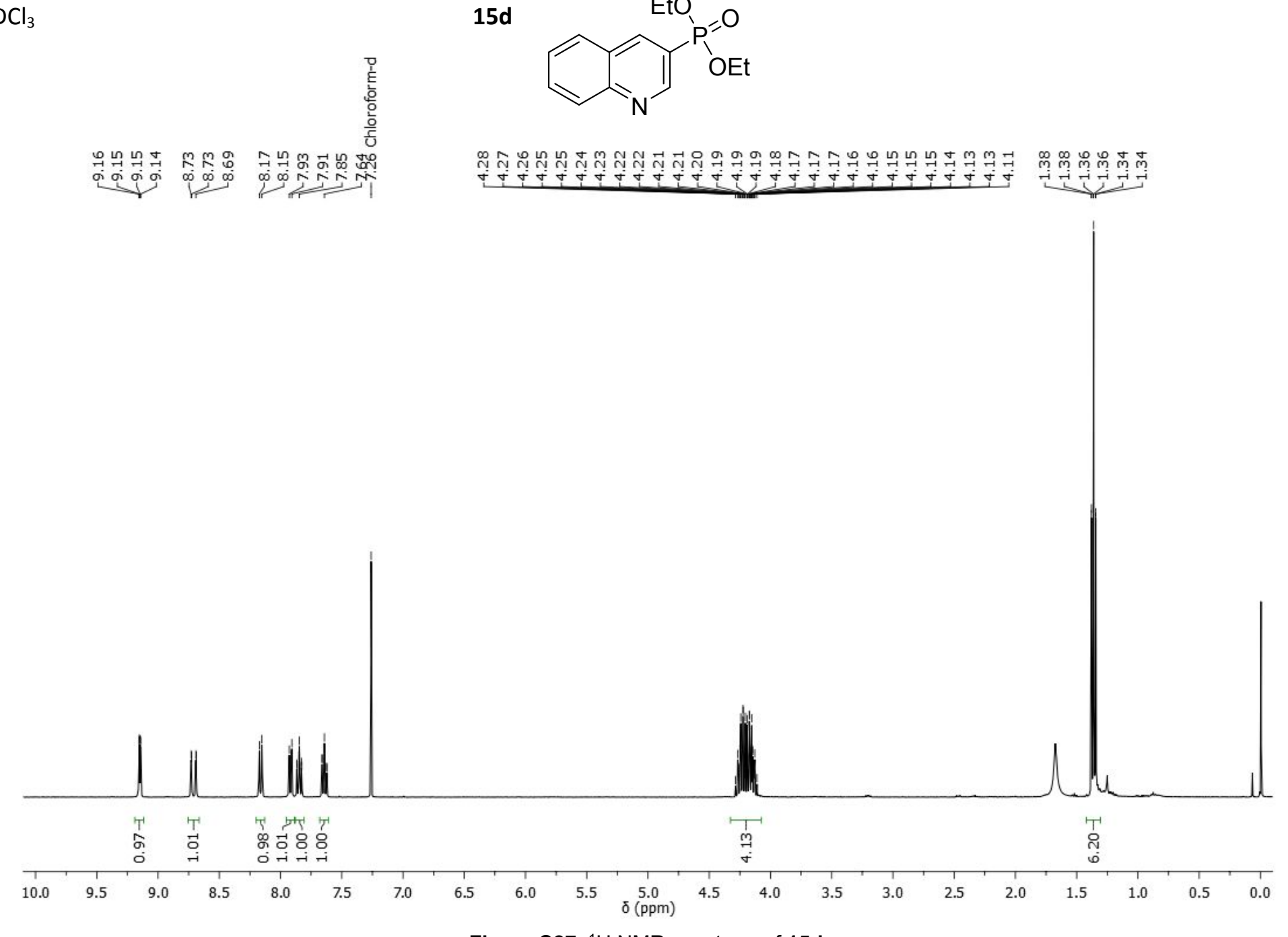

Figure S87. ${ }^{1} \mathrm{H}$ NMR spectrum of $15 \mathrm{~d}$. 


\section{Quinoline phosphonates}

${ }^{13} \mathrm{C} 101 \mathrm{MHz}, \mathrm{CDCl}_{3}$

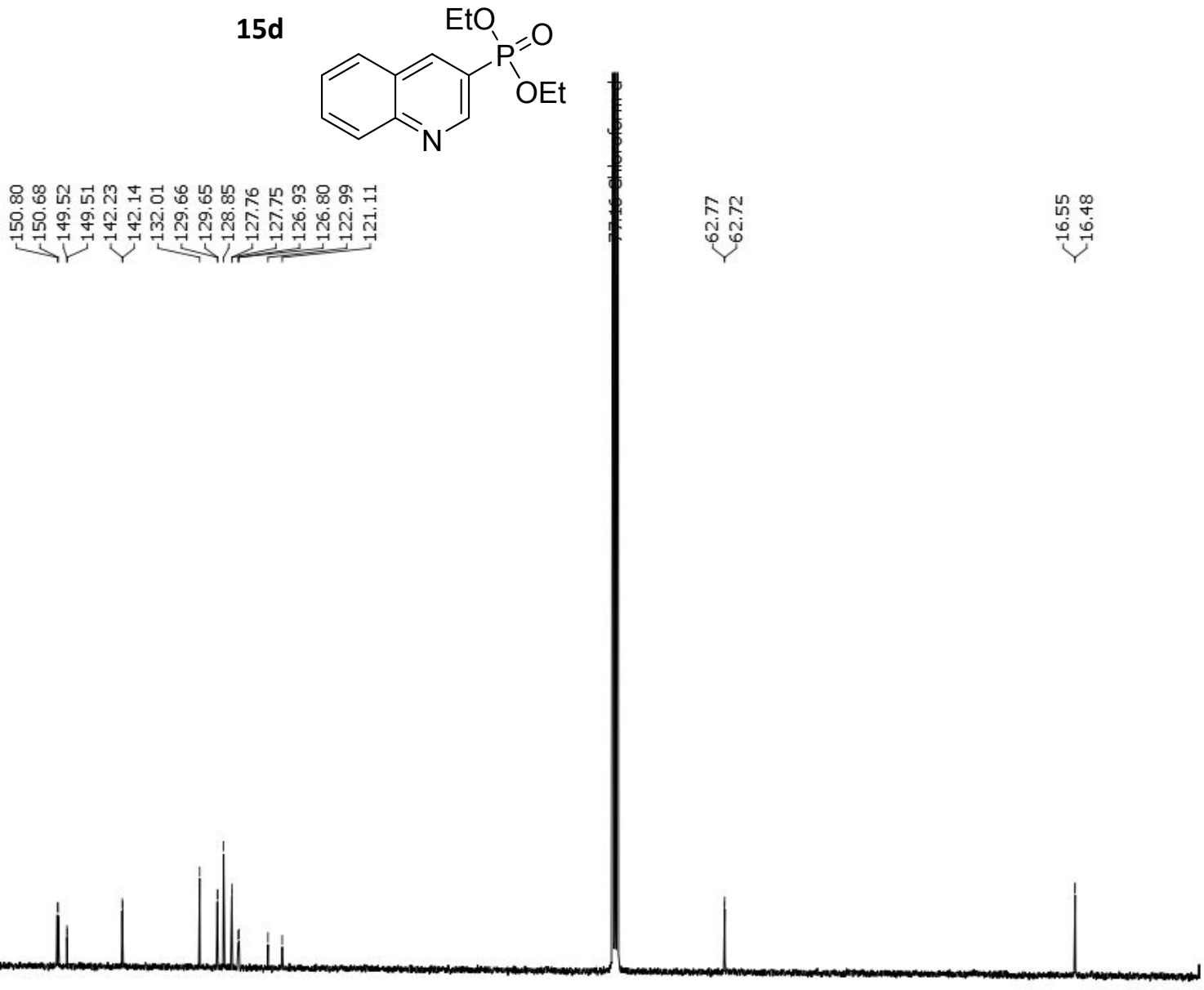

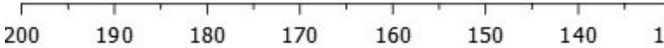

Figure S88. ${ }^{13} \mathrm{C}$ NMR spectrum of $15 \mathrm{~d}$. 
${ }^{1} \mathrm{H} 400 \mathrm{MHz}, \mathrm{CDCl}_{3}$

\section{Indole phosphonate}

$15 \mathrm{e}$

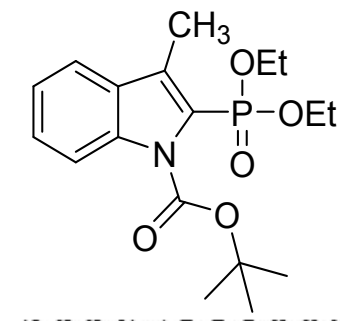

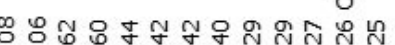

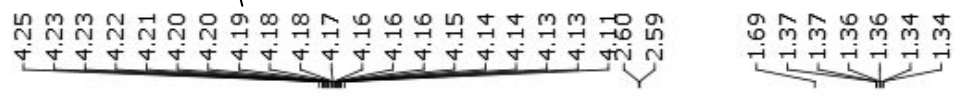

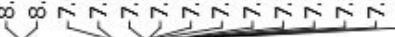

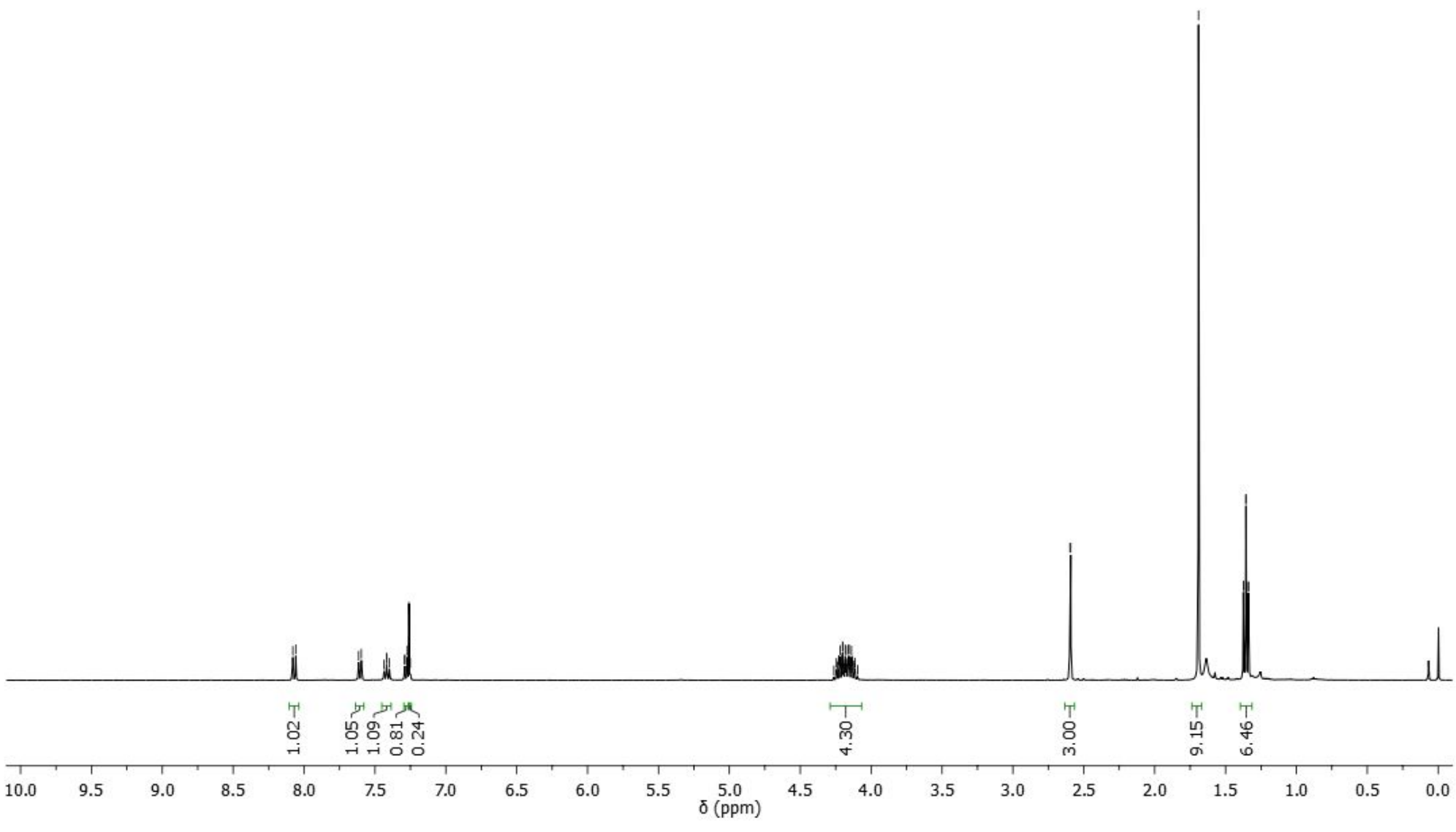

Figure S89. ${ }^{1} \mathrm{H}$ NMR spectrum of $15 e$ 
${ }^{13} \mathrm{C} 101 \mathrm{MHz}, \mathrm{CDCl}_{3}$

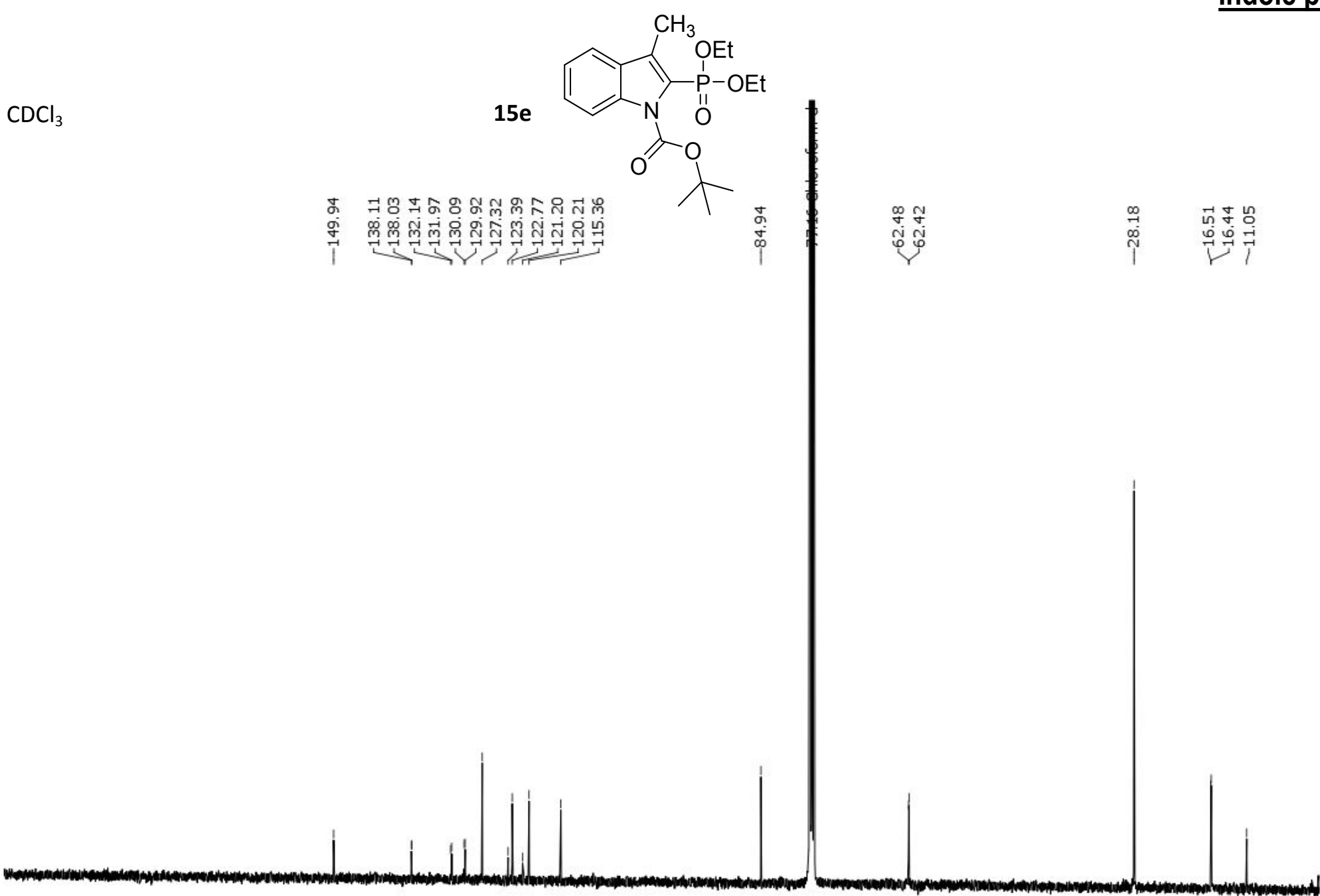

Indole phosphonate

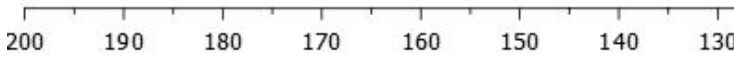

Figure S90. ${ }^{13} \mathrm{C}$ NMR spectrum of $15 e$. 
${ }^{1} \mathrm{H} 400 \mathrm{MHz}, \mathrm{CDCl}_{3}$

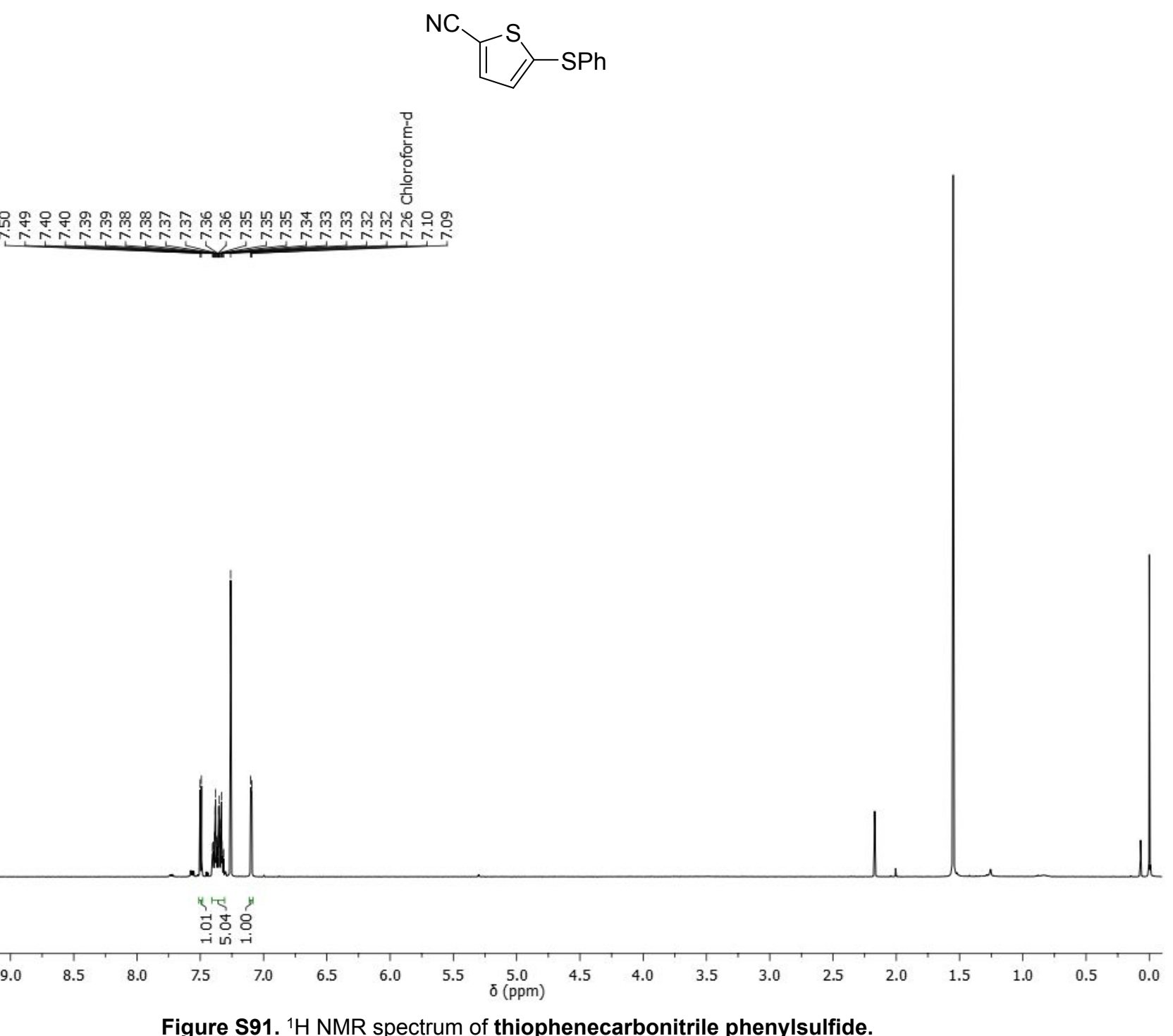

Figure S91. ${ }^{1} \mathrm{H}$ NMR spectrum of thiophenecarbonitrile phenylsulfide. 
Thiophenecarbonitrile phenylsulfide

${ }^{13} \mathrm{C} 101 \mathrm{MHz}, \mathrm{CDCl}_{3}$

${ }^{\mathrm{NC}}{ }^{\mathrm{S}} \mathrm{SPh}$

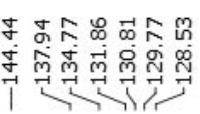

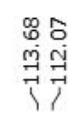
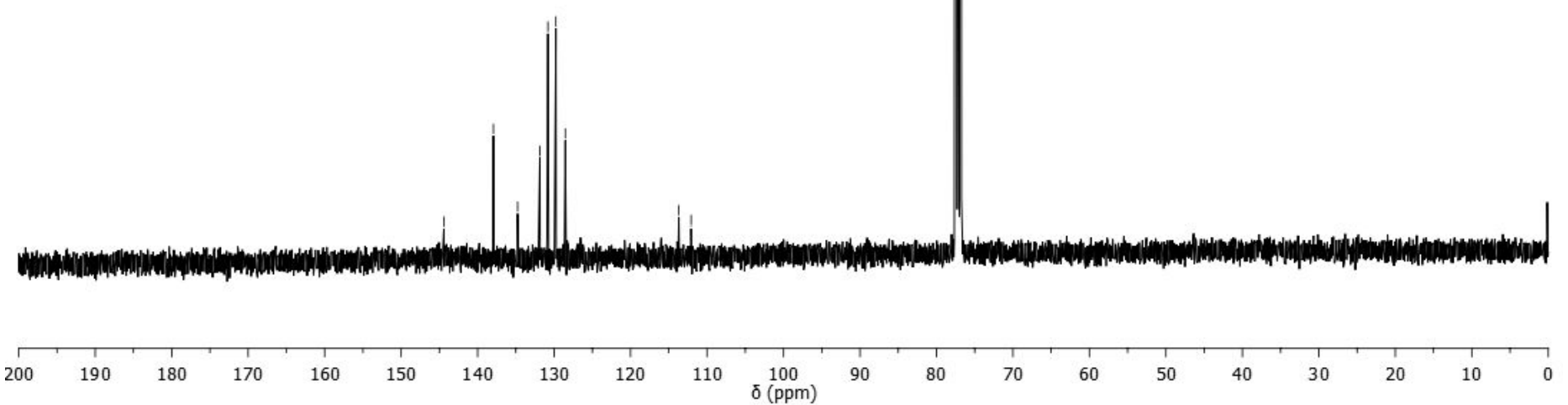

Figure S92. ${ }^{13} \mathrm{C}$ NMR spectrum of thiophenecarbonitrile phenylsulfide.

S114 